\title{
Generation of Nanoparticles by Spark Discharge
}

\author{
Proefschrift
}

ter verkrijging van de graad van doctor

aan de Technische Universiteit Delft

op gezag van de Rector Magnificus,

Prof. dr. ir. J. T. Fokkema, voorzitter van het College voor Promoties,

in het openbaar te verdedigen op

maandag, 19 oktober 2009 om 10:00 uur

door

\section{Nooshin SALMAN TABRIZI}

Masters of Chemical Engineering University of Tarbiat Modarres, Tehran

University of Leeds, Leeds

Geborn te Tehran, Iran 
Dit proefschrift is goedgekeurd door de promotor:

Prof. Dr. A. Schmidt-Ott

Samenstelling promotiecommissie:

Rector Magnificus

Prof. Dr. A. Schmidt-Ott

Prof. Dr. S. J. Picken

Prof. Dr. H. Burtscher

Prof. Dr. P. F. Dunn

Prof. Dr. F. E. Kruis

Dr.S. W. H. Eijt

Dr. van de Krol

Prof. Dr. F. Scarano
Technische Universiteit Delft, voorzitter Technische Universiteit Delft, promotor Technische Universiteit Delft University of Applied Sciences, NW Switzerland University of Notre Dame, Indiana, USA University of Duisburg-Essen, Germany Technische Universiteit Delft Technische Universiteit Delft Technische Universiteit Delft, reservelid

ISBN: 978-90-9024559-1

Copyright (C) 2009 by Nooshin Salman Tabrizi

All rights reserved. No part of the material protected by this copyright notice may be reproduced or utilized in any form or by any means, electronic or mechanical, including photocopying, recording or by any information storage and retrieval system, without written permission from the author. The author encourages the communication of scientific contents and explicitly exempts the use for scientific, non commercial purposes, provided the proper citation of the source. Parts of the thesis are published in scientific journals and copyright is subject to different terms and conditions. 
$\mathscr{T}$
my beloved parents 



\section{Contents}

Introduction 1

1.1 Nanoparticles...................................................

1.1.1 Properties...............................................

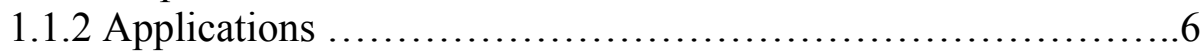

1.1.3 Health Impacts................................................

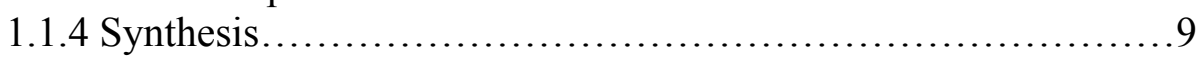

1.2 Thesis Organization.............................................. 10

$2 \quad$ Spark Discharge Generator 14

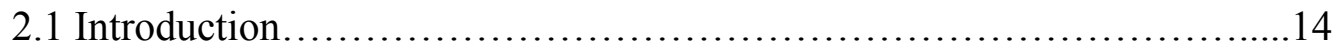

2.1.1 Previous Work........................................... 14

2.1.2 Breakdown in Gas..........................................16

2.2 Spark Discharge Generator........................................ 18

2.2.1 Electrical Circuit............................................. 19

2.2.2 Spark Frequency........................................... 19

2.2.3 Experimental Set-up...................................21

2.3 Results and Discussion ............................................22

2.3.1 Effect of Gas Pressure and Type on the Breakdown Voltage.....22

2.3.2 Effect of Gas Pressure on Particle Formation...................22

2.3.3 Effect of Gas Flow Rate on Particle Formation...................26

2.3.4 Effect of Inter-Electrode Gap on Particle Formation.............27

2.3.5 Effect of Capacitance on Particle Formation....................28

2.3.6 Effect of Electrode Material on Particle Formation..............28

2.3.7 Effect of Spark Repetition Frequency on Particle Formation.....30

2.3.8 Charge State of Particles ........................................31

2.3.9 Particle Losses in the Present Set-up ...........................32

2.3.10 Material Transfer between Electrodes........................32

2.3.11 Aerogel Formation.........................................34

2.4 Conclusion.....................................................

3 Technical Notes on Spark-Generated Particles in View of Applications 40

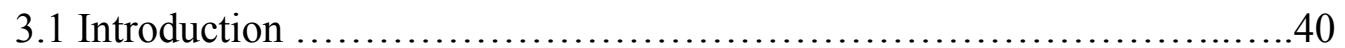

3.2 Experimental Set-up for Particle Characterization........................40

3.3 Particle Characterization Results and Discussion.........................41

3.3.1 Explanation of Large Particle Formation.....................46

3.3.2 Approaches to Suppress Large Particles in the Product..........47

3.3.3 Notes on Oxidation Behavior..............................49

3.4 Examples of Applications...........................................52

3.4.1 Li-Ion Battery.........................................52

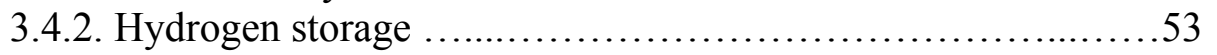

3.4.2.1 Mg Nanoparticles .................................53 
3.4.2.2 Pd Nanoparticles.....................................57

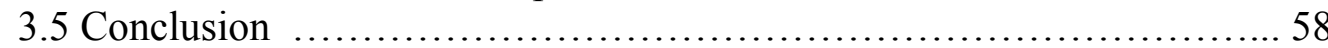

$4 \quad$ Generation of Bimetallic Nanoparticles from Miscible Metals 62

4.1 Introduction.....................................................62

4.2 Experimental Method............................................63

4.3 Results and Discussion...........................................64

4.3.1 Study of Various Systems.................................64

4.3.1.1 Cr-Co.............................................64

4.3.1.2 Au-Pd ...................................... 66

4.3.1.3 Ag-Pd.........................................70

4.3.2 Effect of Various Operating Parameters on Mixing.............74

4.3.2.1 Effect of Capacitance...............................74

4.3.2.2 Effect of Gap Distance.............................75

4.3.2.3 Effect of Spark Frequency........................75

4.3.2.4 Effect of Gas Type.................................76

4.3.2.5 Effect of Gas Flow Rate................................ 77

4.3.3 Compacted electrodes.........................................78

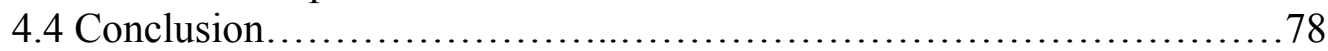

$5 \quad$ Generation of Bimetallic Nanoparticles from Immiscible Metals 82

5.1 Introduction.................................................... 82

5.2 Experiment........................................................ 83

5.3 Results and Discussion......................................... 83

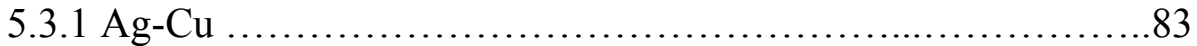

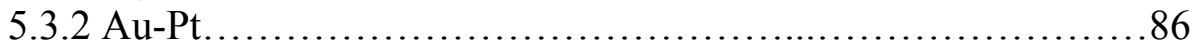

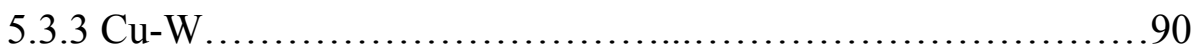

5.4 Conclusion............................................................. 94

$6 \quad$ Theoretical Prediction of primary Particle Size 98

6.1 Introduction......................................................... 98

6.2 Mathematical Model............................................... 100

6.3 Results and Discussion.........................................101

6.4 Conclusion........................................................ 107

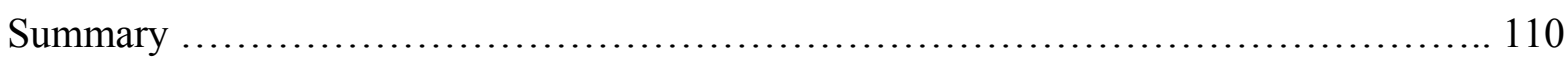

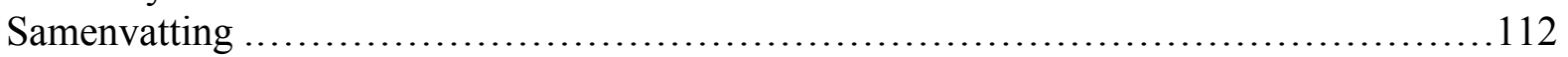

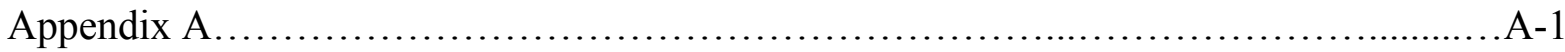

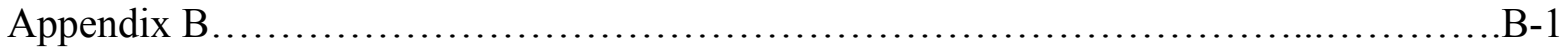

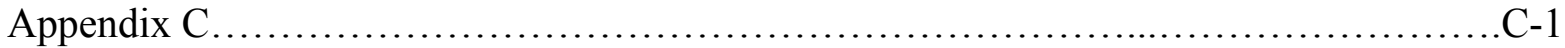

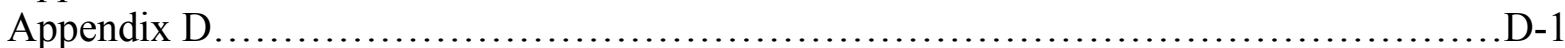

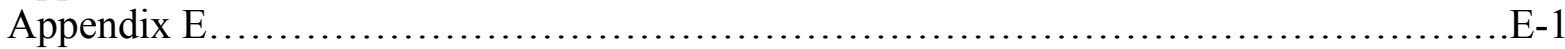

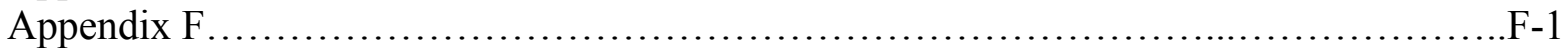




\section{List of Figures}

Figure $1.1 \%$ surface atoms for various particle sizes ......................................................

Figure 1.2 Particle size via total surface area of the particles contained in one mole of Pt.........................4

Figure 1.3 Electronic states in a bulk semiconductor compared with a nanoparticle-based semiconductor ........5

Figure 1.4 Threshold of nanotechnology as basic sciences converge to the nanoscale ..............................6

Figure 1.5 The fractional deposition of particles in human respiratory tract .....................................9

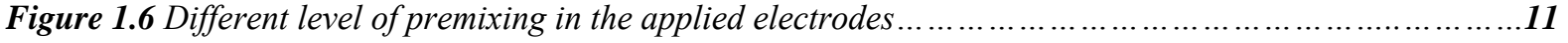

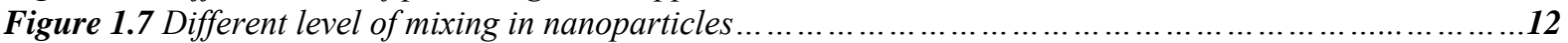

Figure 2.1 Townsend breakdown gap (a), cathode directed streamer(b) ...................................................16

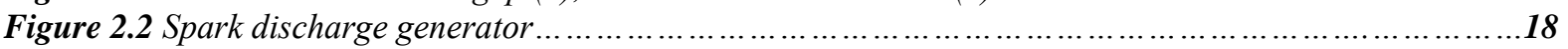

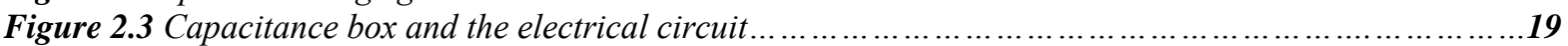

Figure 2.4 Spark frequency (a) and oscillation of the voltage (b), equivalent $R C L$ circuit (c) ...................20

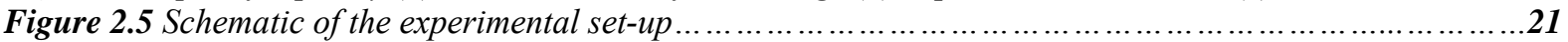

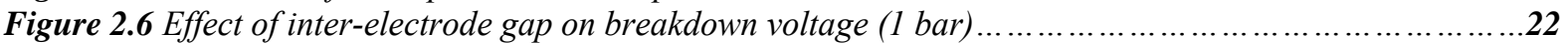

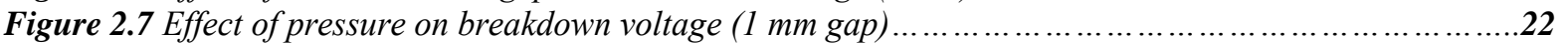

Figure 2.8 TEM micrographs of Au particles produced under Nitrogen, Argon, Helium at 1 and 2 bar..........23

Figure 2.9 Size distributions of particles produced under $N_{2}$ and Ar ........................................24

Figure 2.10 Collision, coalescence, and cooling at early stages of particle growth...............................24

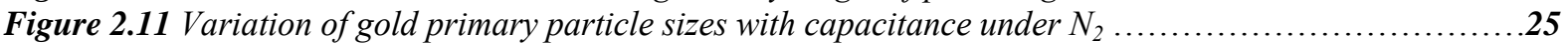

Figure 2.12 Gold particle production rates under $N_{2}$, Ar, He .............................................25

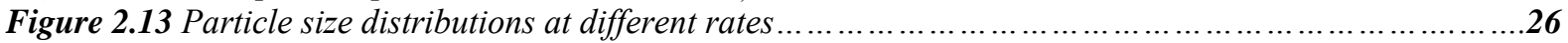

Figure 2.14 Particle modal diameters at various gas flow rates ...............................................26

Figure 2.15 Spark energies at different gap distances under Argon .......................................................27

Figure 2.16 Electrode mass losses per spark at different gap distances................................................27

Figure 2.17 Electrode mass losses per unit spark energy at different gap distances............................27

Figure 2.18 Particle size distributions at different gap distances ..............................................27

Figure 2.19 Effect of capacitance on the particle size distribution ..........................................28

Figure 2.20 Effect of capacitance on mass production rate ................................................28

Figure 2.21 Size distributions of particles produced from various materials ...................................30

Figure. 2.22 Electrode material losses for various electrodes ........................................................30

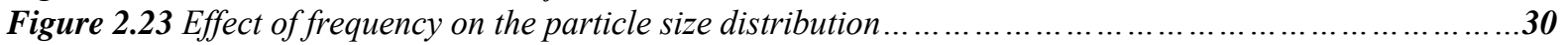

Figure 2.24 Effect of frequency on gold particle production rate ..............................................30

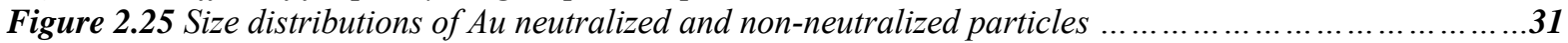

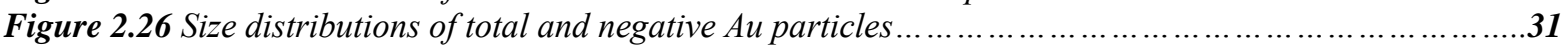

Figure 2.27 Size distributions of Ag neutralized and non-neutralized particles....................................32

Figure 2.28 Size distributions of total and negative $\mathrm{Ag}$ particles .............................................32

Figure 2.29 Image converter photographs of 600-amp, 3- $\mu$ sec spark between Zn electrodes.......................33

Figure 2.30 Material transfer from $\mathrm{Cu}$ electrode to $\mathrm{W}$ electrode (a), $\mathrm{Cu}$ electrode erosion (b) .......................33

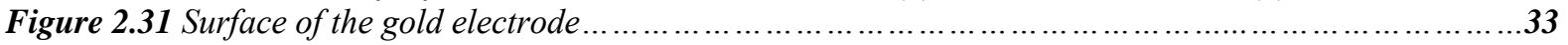

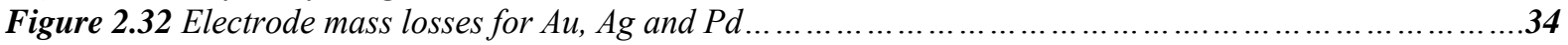

Figure 2.33 Self-assembly of carbon nanoparticles (a), SEM micrographs of Pd web assembly (b) ...............35

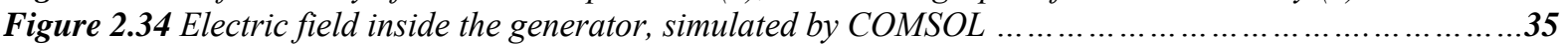

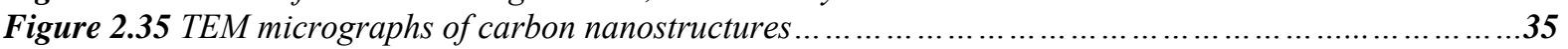

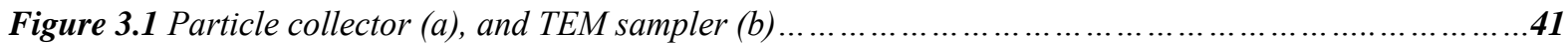

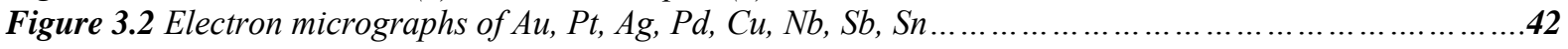

Figure 3.3 A typical size histogram of metallic nanoparticles generated by the spark discharge .................43

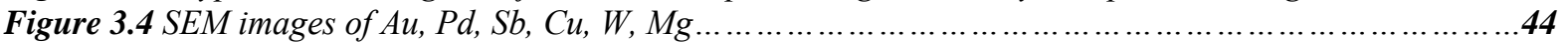

Figure 3.5 Electron micrographs displaying sputtered large particles of Au ..................................46

Figure 3.6 Formation mechanism of large particle from electrode surface .....................................46

Figure 3.7 Cu-W particles produced from compositionally different electrodes of $\mathrm{Cu}$ and $W \ldots \ldots \ldots \ldots \ldots \ldots \ldots . . . . . . . . . . .47$

Figure 3.8 Deposition of particles on the precipitator plates and SEM images of the particles....................48

Figure 3.9 Magnesium hollow electrode .................................................................49

Figure 3.10 Mg particles using hollow electrode as gas inlet (a), and gas outlet (b) ...........................49 
Figure 3.11 TEM micrograph of Mg particles (a), XRD pattern of the $\mathrm{Mg}$ sample(b) ............................50

Figure $3.12 \mathrm{Mg}$ atomic percent vs particle size at different oxide layer thicknesses $(d)$..........................51

Figure 3.13 Closed loop for producing unoxidized particles (a), burned Sb particles upon exposure to air (b).51

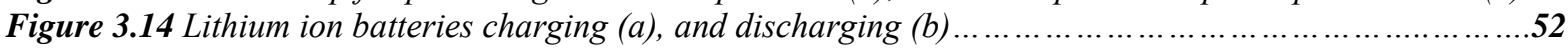

Figure 3.15 Born-Haber cycle for $\mathrm{Mg}-\mathrm{H}_{2}$ system at standard pressure and temperature ...........................54

Figure 3.16 Crystal structure of $\mathrm{MgH}_{2}(\mathrm{a})$, TEM image of $\mathrm{Mg}$ nanoparticles (b), black $\mathrm{Mg}$ deposited on the

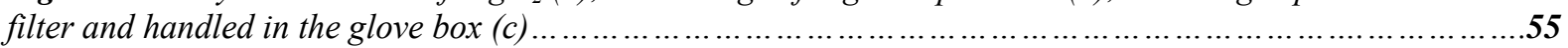

Figure 3.17 Sample holder (a), thermal deposition setup for hydrogen desorption measurements (b) .............56

Figure 3.18 Hydrogen desorption measurements of $\mathrm{Mg}$ nanoparticles .......................................56

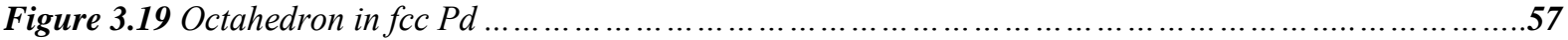

Figure 3.20 Variation of pressure vs. temperature for Pd nanoparticles.........................................58

Figure 3.21 Accumulative hydrogen desorption measurement of Pd nanoparticles...............................58

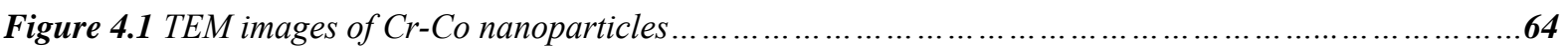

Figure 4.2 EDS spectrum of $\mathrm{Cr}$-Co nanoparticles ..........................................................6.

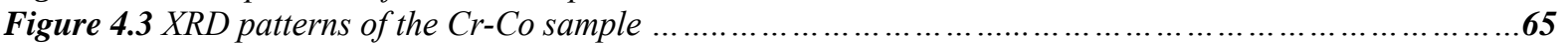

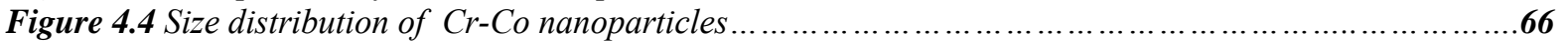

Figure 4.5 TEM and SEM images of Au-Pd nanoparticles ....................................................67

Figure 4.6 Typical STEM maps of a Au-Pd particle (a), AuL (b), PdL (c) ....................................68

Figure 4.7 XRD patterns of the $A u, P d,[A u(+) P d(-)]$, and $[A u(-) P d(+)]$ nanoparticles..........................69

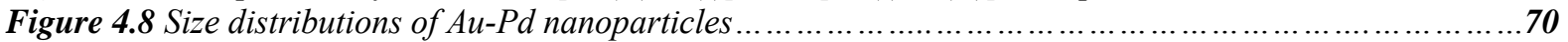

Figure 4.9 TEM micrograph (left) and STEM image from less concentrated region of the sample (right).......71

Figure 4.10 Typical STEM maps of a Ag-Pd particle (a): AgL, (b): PdL, (c) ..................................71

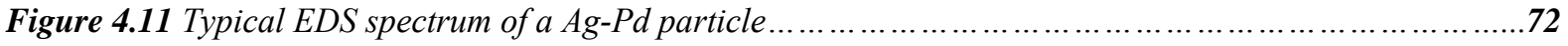

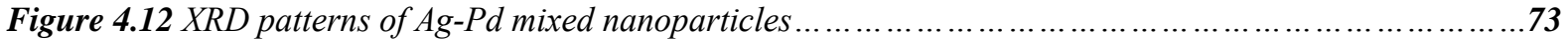

Figure 4.13 Size distributions of the mixed $\mathrm{Ag}$-Pd nanoparticles ................................................73

Figure 4.14 XRD patterns of the mixed phases at various capacitances.........................................74

Figure 4.15 XRD patterns of the mixed phases at various gap distances ......................................75

Figure 4.16 XRD patterns of the mixed phases at various frequencies ......................................76

Figure 4.17 XRD patterns of the mixed phases at different gas types ..........................................76

Figure $4.18 X R D$ patterns of the mixed phases at various gas flow rates ..........................................77

Figure 4.19 Schematic of Magnetic Pulse Compaction (a), and compacted electrode fabricated by MPC (b)...78

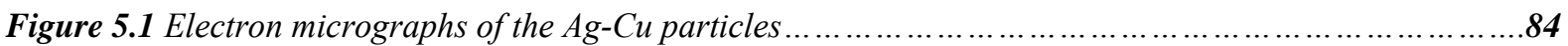

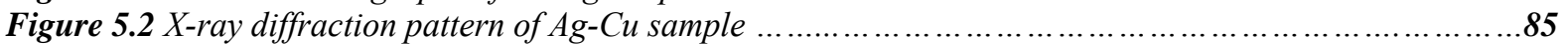

Figure 5.3 Size distributions of $\mathrm{Ag}, \mathrm{Cu}$ and $\mathrm{Ag}$-Cu nanoparticles..........................................86

Figure 5.4 TEM images of Au (a), Pt (b), and Au-Pt (c) nanoparticles......................................86

Figure 5.5 EDS compositional mapping of the specified Au-Pt particle $\sim 6 \mathrm{~nm}$ in size (a); Au L (b); Pt L (c)....87

Figure 5.6 EDS compositional mapping of the specified Au-Pt particle (a); Au L (b); Pt L (c) ..................88

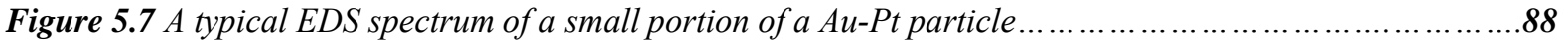

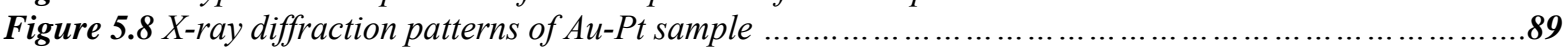

Figure 5.9 TEM image of the $\mathrm{Cu} / W$ nanoparticles (a), SEM image of the $C u / W$ particles.........................90

Figure 5.10 A typical EDS spectrum recorded from a small portion of a $\mathrm{Cu}-\mathrm{W}$ particle.........................91

Figure 5.11 Electron micrographs of the $\mathrm{Cu}$-W particles with EDS line scans ................................91

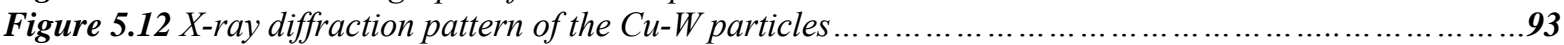

Figure 5.13 Electron diffraction patterns of the specified particles.......................................93

Figure 5.14 Size distributions of $\mathrm{Cu}, W$ and $\mathrm{Cu}$-W nanoparticles.......................................94

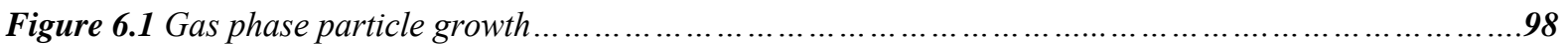

Figure 6.2 Coalescence in nanoparticles ..........................................................99

Figure 6.3 Coalescence and collision characteristic times for different oxides in typical operating conditions of

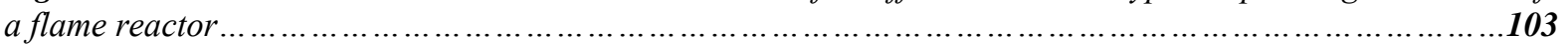

Figure 6.4 Particle size calculation for Titania ...........................................................103

Figure 6.5 Variation of primary particle size of different oxides with cooling rate ...............................104

Figure 6.6 Relative particle sizes at different cooling rates..............................................104

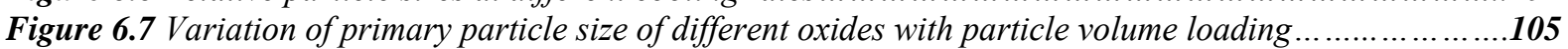

Figure 6.8 Schlieren photograph taken at $210 \mu \mathrm{s}$ with respect to the onset of current in the spark...............106 
Figure 6.9 Primary particle size predicted for Au....

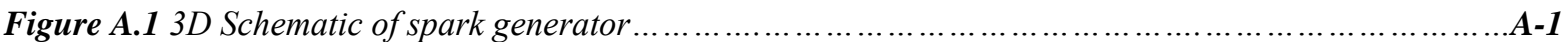

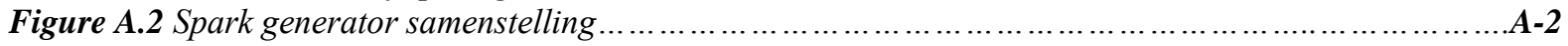

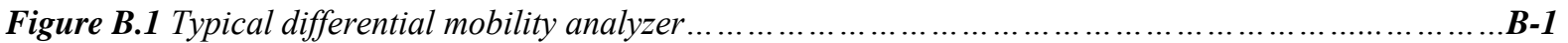

Figure B.2 Particle sizes selected at different applied voltage on the short DMA .............................

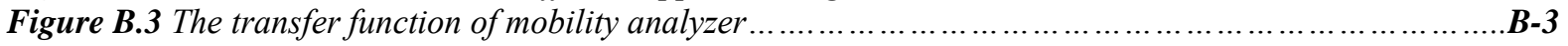

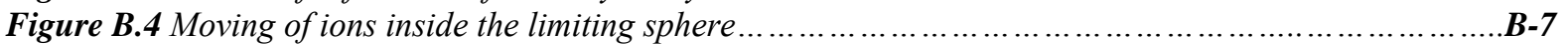

Figure B.5 Fraction of particles carrying one charge exposed to bipolar ions in Argon ...........................-10

Figure B.6 Fraction of particles carrying one charge exposed to bipolar ions in Nitrogen........................10

Figure B.7 Fraction of particles carrying one charge in equilibrium with bipolar ions in air.....................11

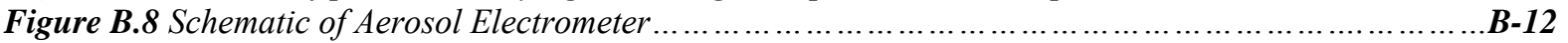

Figure B.9 Electric circuit of Aerosol Electrometer .....................................................12

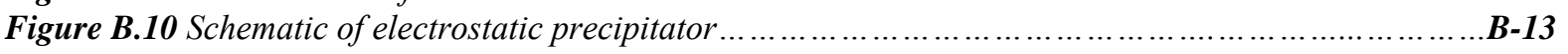

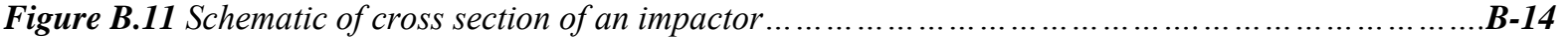

Figure C.1 Schematic phase diagram for a typical metal ...................................................

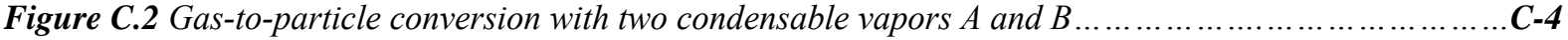

Figure D.1 Volumetric and gravimetric hydrogen density of some hydrides ..................................D-2

Figure D.2 A typical Pressure-Concentration-Isotherms plot for a metal hydride and the Van 'tHoff curve...D-3

Figure E.1 Simple (a), body centered (b), faced centered (c) cubic structures ..................................1

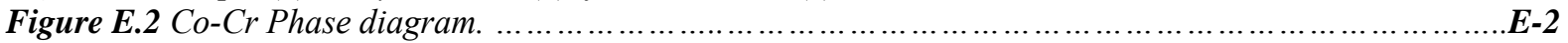

Figure E.3 Co-Cr Lattice parameter vs. composition for $f c c(\alpha-C o)$ solid solutions ..................................2

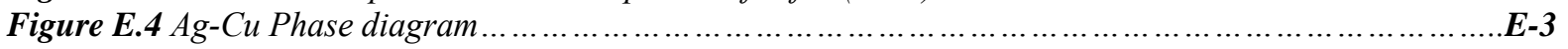

Figure E.5 Ag-Cu Lattice parameter vs. composition for the metastable solid solution ...............................

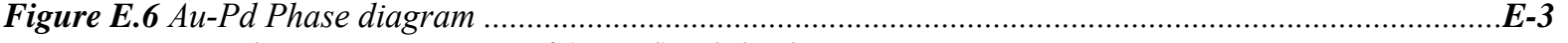

Figure E.7 Au-Pd Lattice parameter of $(A u-P d)$ solid solution vs. Composition..............................................

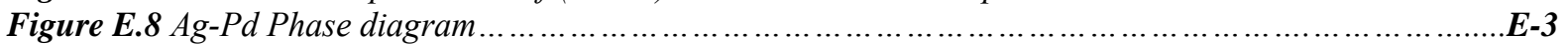

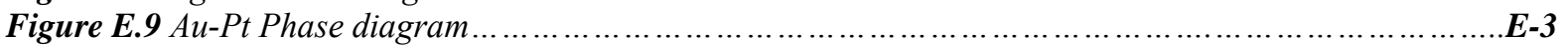

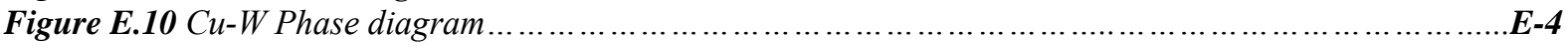




\section{List of Tables}

Table 1.1 Diagram showing the atom number and size scale of various particles ..................................2

Table 1.2 Number of atoms in metallic nanoparticles with fcc structures ............................................

Table 1.3 General effects of decreasing particle size ...............................................................

Table 1.4 Size effect in magnetic properties of some metals................................................ 5

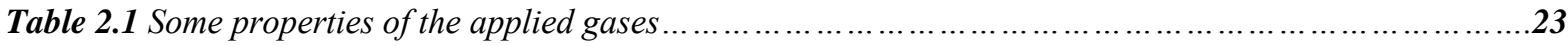

Table 2.2 Gold particle sizes under various atmospheres .................................................24

Table 3.1 Specific surface areas of particles produced from different electrodes ...............................45

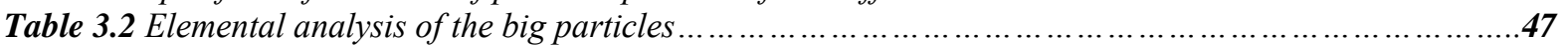

Table 4.1 Composition of $\mathrm{Cr}$-Co in the electrodes and in the sample measured by ICP..........................65

Table 4.2 Specific surface area of $\mathrm{Cr}$-Co nanoparticles.......................................................66

Table 4.3 Average compositions of Pd-Au samples measured by ICP ..........................................67

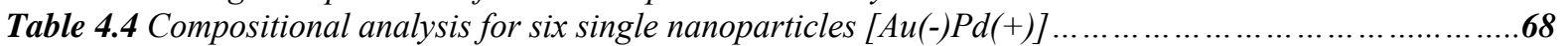

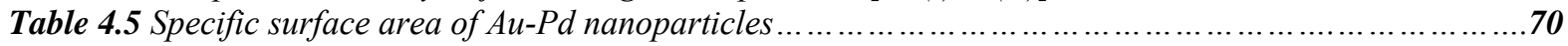

Table 4.6 Average composition of Ag-Pd samples measured by ICP .........................................71

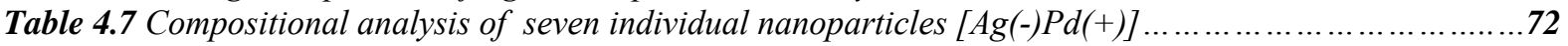

Table 4.8 Specific surface area of Ag-Pd nanoparticles...........................................................73

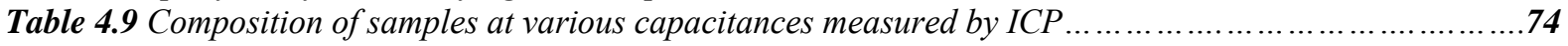

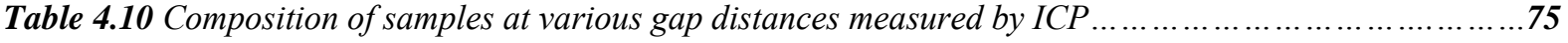

Table 4.11 Composition of samples at various frequencies measured by ICP ...................................76

Table 4.12 Composition of samples at different gas types measured by ICP .......................................77

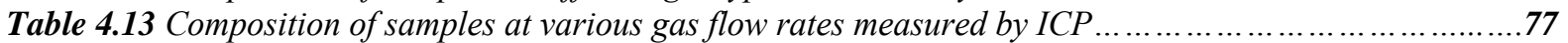

Table 5.1 Compositional analysis of Ag-Cu sample measured by ICP ..........................................84

Table 5.2 Specific surface area of the Ag-Cu particles .........................................................8

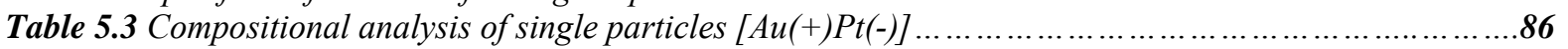

Table 5.4 Compositional analysis of Au-Pt samples measured by ICP ......................................88

Table 5.5 Specific surface area of the Au-Pt particles.........................................................90

Table 5.6 Typical atomic compositions of the $\mathrm{Cu}$-W particles..................................................91

Table 5.7 Compositional analysis of $\mathrm{Cu}-\mathrm{W}$ sample measured by XPS .........................................91

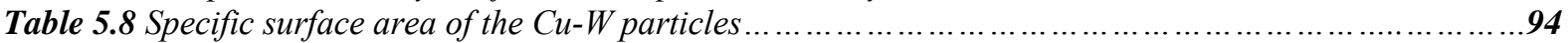

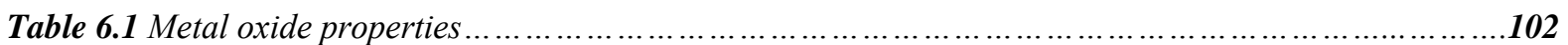

Table 6.2 Model predictions and experimental data from Flame reactor ....................................103

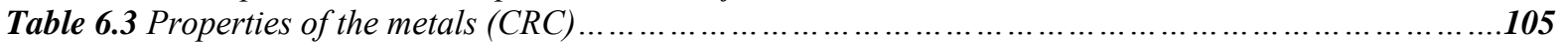

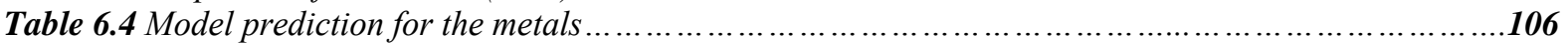

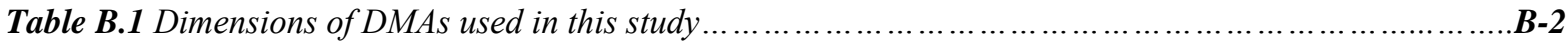

Table B.2 Comparison of calculated $(\alpha)$ coefficients in this work (top) with Fuch 's calculations..................B-7

Table B.3 Comparison of the stationary charge distribution on particles calculated in this work (top) with

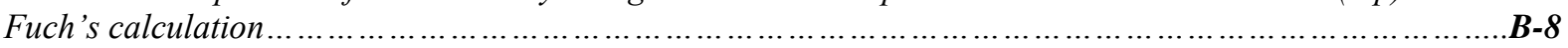

Table B.4 Calculated positive and negative ions properties in different gases....................................9

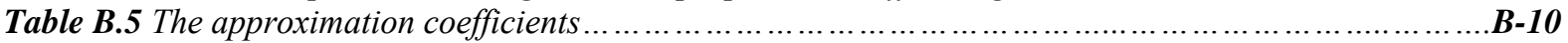

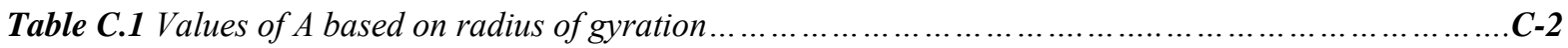

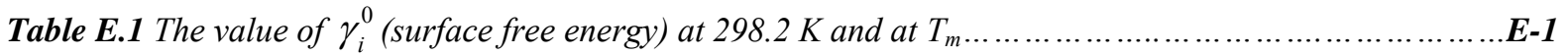

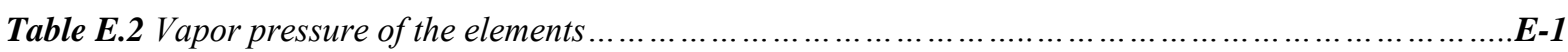

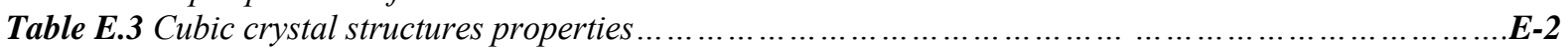




\section{Chapter 1}

\section{Introduction}

\subsection{Nanoparticles}

Particles with dimensions smaller than $100 \mathrm{~nm}\left(10^{-9} \mathrm{~m}\right)$ are generally termed nanoparticles. One cubic nanometer is roughly 20 times the volume of an individual atom. Small nanoparticles containing fewer than $10^{4}$ molecules or atoms are referred to as clusters. Table 1.1 gives an idea of particle size range and the number of atoms they contain (Yacamán et al., 2001). Since Heisenberg's Uncertainty Principle ${ }^{1}$, applies only to the subatomic particles, the quantum mechanical calculation by this principle places no limit on how well atoms and molecules can be held in place (Mansoori, 2005).

If the primary particles contact each other at surfaces and edges, they form aggregates with probably ionic, covalent or metallic bonds which can not be broken down further by shear forces. When the particles contact each other at points, they form agglomerates with weak bonds which may be due to van der Waals forces or ionic, covalent bonds operating over small contact area. The particle properties are determined by the laws which are dependent on the size of the particles. In addition to size, other important characteristics of nanoparticles are composition, morphology, distribution of phases in the case of multi-phases, and the nature of the interfaces.

In crystalline solids, the atoms are arranged in a long-range order throughout the crystal. However in amorphous materials (i.e. glass, wax), the atoms have short-range order and the regularity persists only over unappreciable distances.

In the solid state, metals mostly form close-pack lattices; thus $\mathrm{Ag}, \mathrm{Al}, \mathrm{Au}, \mathrm{Co}, \mathrm{Cu}, \mathrm{Pb}, \mathrm{Pt}$ and $\mathrm{Rh}$ are face-centered cubic (fcc) and $\mathrm{Mg}, \mathrm{Nd}, \mathrm{Os}, \mathrm{Re}, \mathrm{Ru}, \mathrm{Y}$ and $\mathrm{Zn}$ are hexagonal closepacked (hcp). Some other metallic atoms crystallize in body-centered (bcc) lattice, and few such as $\mathrm{Cr}$, $\mathrm{Li}$ and $\mathrm{Sr}$, depending on temperature, crystallize in all three structure types (Poole, 2003). In close packed structures, an atom has 12 nearest neighbors. For fcc lattice, these 13 atoms can be considered as the smallest theoretical nanoparticle. If these 13 atoms are covered by a layer of 42 atoms, a 55-atom nanoparticle with the same shape will be obtained. In this way, by adding more layers, larger particles can be formed all having the same shape. In this fcc nanoparticle, for $(\mathrm{n})$ layers the number of atoms $(\mathrm{N})$ called structural magic numbers, is given by the formula (Poole,2003):

$$
N=\frac{1}{3}\left[10 n^{3}-15 n^{2}+11 n-3\right]
$$

and the number of atoms on the surface $\mathrm{N}_{\text {surf }}$ is:

$$
N_{\text {surf }}=10 n^{2}-20 n+12
$$

\footnotetext{
${ }^{1}$ Quantum mechanical Heisenberg's Uncertainty Principle states that the position and momentum of an object can not simultaneously and precisely be determined.
} 
Table 1.1 Diagram showing the atom number and size scale of various particles (by courtesy of Yacamán et al., 2001)

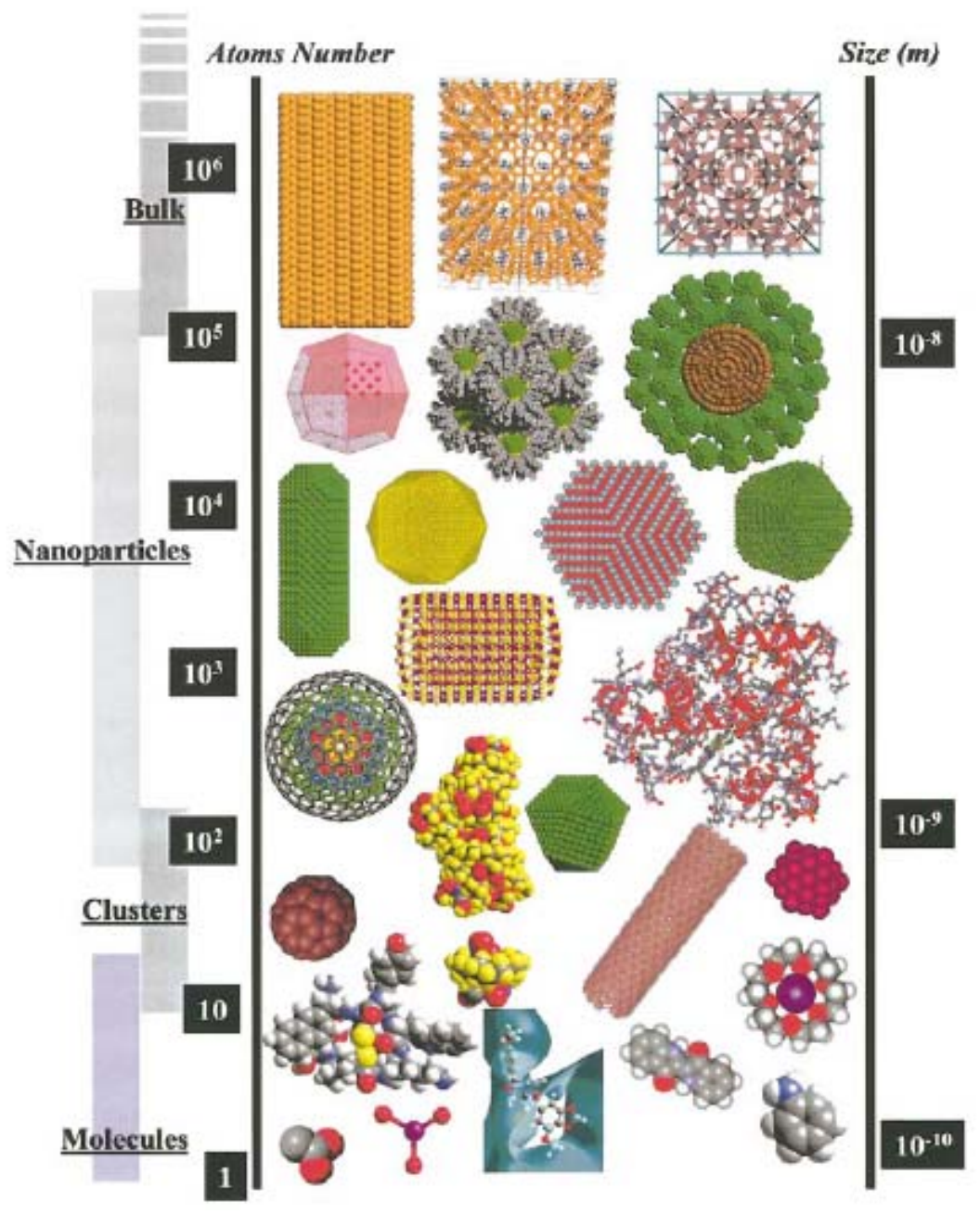

and the diameter of the particle is (Poole,2003):

$d_{\text {particle }}=(2 n-1) d$

$d=a / \sqrt{2}$

where $\mathrm{d}$ is the distance between the centers of nearest-neighbor atoms, and a is the lattice constant.

Table 1.2 lists the total number of atoms, as well as the percentage of atoms on the particle surface in metallic nanoparticles with fcc structures (Poole, 2003). Sequences in cluster formation are intimately related to the nucleation and growth processes, which are governed by the often competing bonding and packing factors. While these processes may be kinetically or thermodynamically controlled, and hence sensitive to experimental conditions, 
the fact that bonding effects are electronic in origin and packing factors are steric in nature implies that magic sequences result from successive fillings of electronic or atomic shells, respectively (Feldheim, 2002).

Table 1.2 Number of atoms in metallic nanoparticles with fcc structures (reproduced from Poole, 2003)

\begin{tabular}{|l|l|l|l|l|}
\hline Shell number & Diameter & \multicolumn{3}{|l|}{ Number of fcc nanoparticle atoms } \\
\cline { 3 - 5 } & & Total & On surface & $\%$ Surface \\
\hline 1 & $1 \mathrm{~d}$ & 1 & 1 & 100 \\
\hline 2 & $3 \mathrm{~d}$ & 13 & 12 & 92.3 \\
\hline 3 & $5 \mathrm{~d}$ & 55 & 42 & 76.4 \\
\hline 4 & $7 \mathrm{~d}$ & 147 & 92 & 62.6 \\
\hline 5 & $9 \mathrm{~d}$ & 309 & 162 & 52.4 \\
\hline 6 & $11 \mathrm{~d}$ & 561 & 252 & 44.9 \\
\hline 7 & $13 \mathrm{~d}$ & 923 & 362 & 39.2 \\
\hline 8 & $15 \mathrm{~d}$ & 1415 & 492 & 34.8 \\
\hline 9 & $17 \mathrm{~d}$ & 2057 & 642 & 31.2 \\
\hline 10 & $19 \mathrm{~d}$ & 2869 & 812 & 28.3 \\
\hline 11 & $21 \mathrm{~d}$ & 3871 & 1002 & 25.9 \\
\hline 12 & $23 \mathrm{~d}$ & 5083 & 1212 & 23.8 \\
\hline
\end{tabular}

$d$ for Al 0.286, Au 0.288, Cu 0.256, Fe 0.248, Pb 0.350, Pd 0.275

Due to the nature of the forces such as surface tension, nanoparticles may assume shapes and structures different from those of bulk material, and shape is not necessarily constant because the energy of nanoparticles may show many local minima configurations, corresponding to different structures, where a small excitation may be sufficient to induce transitions on the particles (Yacamán et al., 2001).

Usually as a surface energy becomes a minimum, the polycrystalline particles and amorphous particles become spherical in shape (Waseda, 2004). Crystalline particles smaller than $20 \mathrm{~nm}$ look almost spherical while larger ones often display pronounced crystal habits (Granqvist, 1976).

If particles are non-spherical, an equivalent diameter is defined that is the diameter of the sphere having the same value of a particular physical property as that of irregular particle. Average properties of particles can be approximated by integrating over the size distribution.

\subsubsection{Properties}

Small sizes of nanoparticles imply quite a high population atoms or molecules located on the surface. For example, as can be seen in Figure 1.1, a fcc metallic particle of $1.5 \mathrm{~nm}$ size has $76.4 \%$ atoms on the surface and since these surface atoms are chemically unsaturated they introduce special properties which differ from bulk properties (Waseda, 2004). The difference can also be partially due to volume effects in which the number of atoms composing a particle is insufficient for bulk properties to manifest (Wu et al., 1993).

The high surface to volume ratio in nanoparticles provides a tremendous driving force for diffusion, especially at elevated temperatures so sintering can take place at lower temperatures, over shorter time scales than for larger particles.

The surface free energy affects the chemical potential and hence the thermodynamic properties. A simple example is the reduced melting point of nano-scaled particles (Goldstein, 1997). 


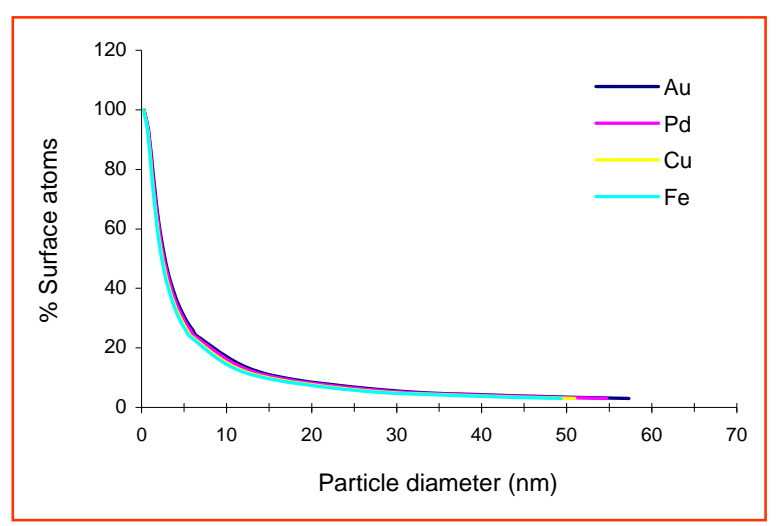

Figure $1.1 \%$ surface atoms for various particle sizes

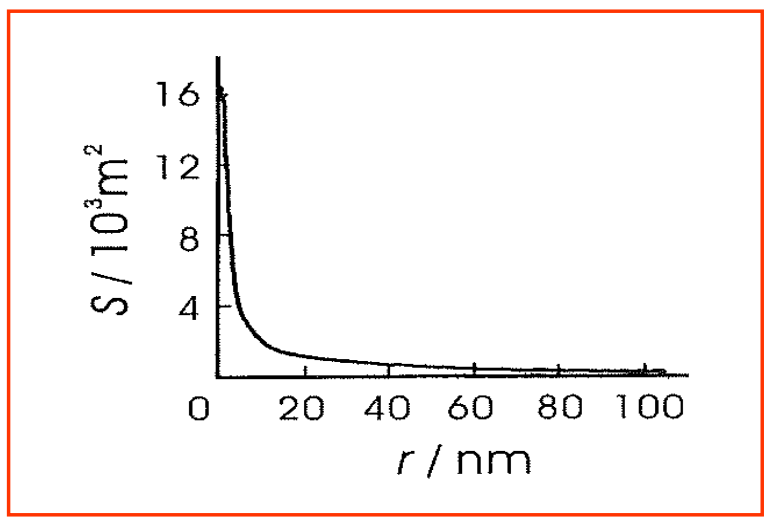

Figure 1.2 Particle size via total surface area of the particles contained in one mole of Pt (from Waseda, 2004)

Nanoparticles show an increased solid-solid phase transition pressure, a lower effective Debye temperature $^{2}$, a decreased ferroelectric phase transition temperature (Kruis, 1998), and modified physical properties (Table 1.3). For example, copper nanoparticles smaller than 50 $\mathrm{nm}$ are considered super hard materials that do not exhibit the same malleability and ductility as bulk copper. Pellets made of small Au and Pd nanocrystals exhibit nonmetallic behavior with specific conductivities in the range of $10^{-6} \Omega^{-1} \mathrm{~cm}^{-1}$. An increase in the diameter of the nanocrystals, however, increases dramatically the conductivity (Schwarz, 2004).

Table 1.3 General effects of decreasing particle size (Gutsch, 2002)
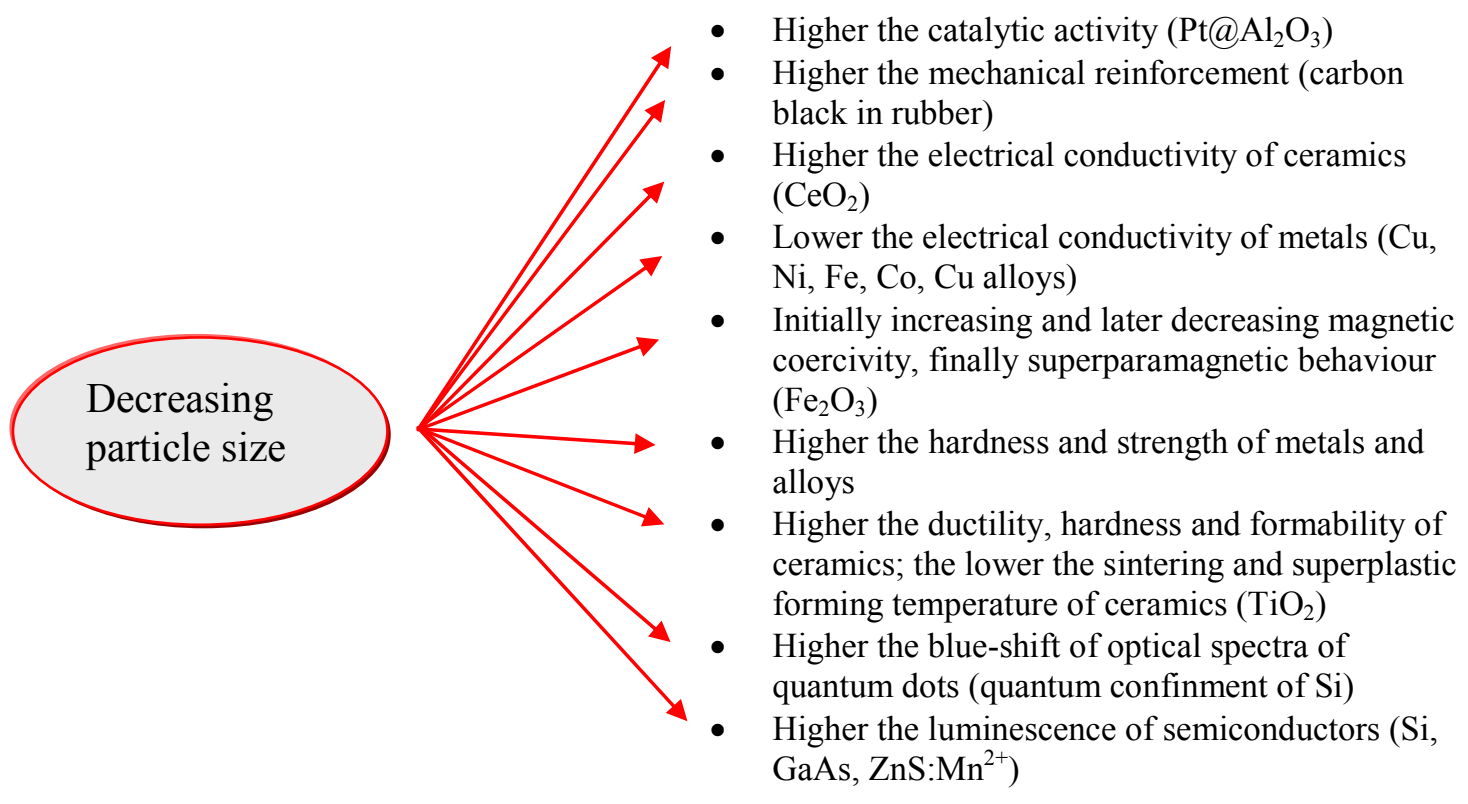

Semiconductors are a unique class of materials which can exhibit characteristic properties of both metals and insulators depending on conditions that determine the electronic nature of the valance and conduction bands. In the ground state, the valance band is completely filled and separated from the conduction band by a narrow band gap $\left(E_{p}\right)$. When sufficient energy is

\footnotetext{
${ }^{2}$ Debye temperature is the temperature of a crystal's highest normal mode of vibration
} 
applied to a semiconductor, it becomes conducting by excitation of electrons from the valence band into the conduction band. This excitation process leaves holes in the valance band, and thus creates electron-hole pairs. In the presence of an external electric field, the electron and the hole will migrate in the conduction and valance bands, respectively. Reducing the size of semiconductors to nano-scale leads to obtaining a fascinating class of novel materials that exhibit properties of quantum confinement (Rotello, 2004). This is observed in nanoparticles with radii smaller than the average distance between the electron and the hole, known as the bulk exciton Bohr radius (e. g. approximately $11 \mathrm{~nm}$ in the case of CdSe nanocrystals). Thus, nano-sized semiconductors show larger band gaps relative to the bulk material and have discrete excitable states (Rotello, 2004).

Band gap energies of nanoscopic semiconductors are strongly size dependent, such that as the radius of the nanocrystal decreases, the band gap increases. This allows tuning the emission wavelength of the nanoparticle by adjusting its size within the quantum-confined regime (Rotello, 2004).

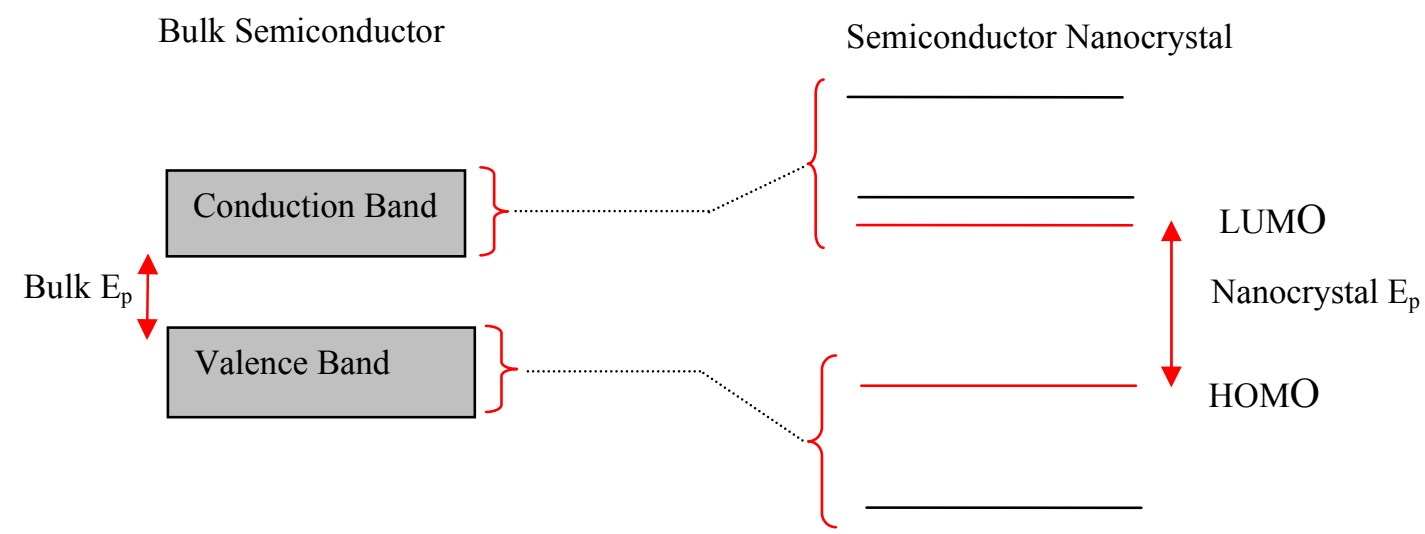

Figure 1.3 Electronic states in a bulk semiconductor compared with a nanoparticle-based semiconductor (HOMO denotes highest occupied molecular orbital, LUMO denotes lowest unoccupied molecular orbital), (reproduced from Rotello, 2004)

The change in properties is not always desirable. Ferroelectric materials smaller than $10 \mathrm{~nm}$ can switch their magnetisation direction using room temperature thermal energy, thus making them useless for memory storage. Small particles of magnetic and even nonmagnetic solids exhibit a totally new class of magnetic properties marked by quantum effects (Table 1.4).

Table 1.4 Size effect in magnetic properties of some metals (Edelstein-1996)

\begin{tabular}{lll}
\hline Metal & Bulk & Cluster \\
\hline $\mathrm{Na}, \mathrm{K}$ & Paramagnetic & Ferromagnetic \\
$\mathrm{Fe}, \mathrm{Co}, \mathrm{Ni}$ & Ferromagnetic & Superparamagnetic \\
$\mathrm{Gd}, \mathrm{Tb}$ & Ferromagnetic & Rotors/ superparamagnetic \\
$\mathrm{Cr}$ & Antiferromagnetic & Frustrated paramagnetic \\
$\mathrm{Rh}$ & Paramagnetic & Ferromagnetic \\
\hline \multicolumn{2}{c}{ Cluster sizes 2 to 300 atoms }
\end{tabular}

Ferromagnetic and antiferromagnetic multilayers have found to exhibit giant magnetoresistance (GMR), which arises because of the differences in spin dependent electron scattering from the ferromagnetic and antiferromagnetic layers (Goldstein, 1997). This effect can also occur in particle precipitates of a magnetic material in a non-magnetic matrix. The smaller the particle size, the bigger the GMR effect is. It is a large decrease in the electrical 
resistivity of certain materials when exposed to magnetic fields. GMR is observed in immiscible metals, such as $\mathrm{Co}-\mathrm{Cu}, \mathrm{Fe}-\mathrm{Ag}, \mathrm{Fe}-\mathrm{Cu}, \mathrm{Fe}-\mathrm{Au}$, and $\mathrm{Co}-\mathrm{Ag}$ (Kruis, 1998).

The electronic quantity most easily accessible to experiments is the ionization potential. The studies on ionization potential of clusters of various elements show that the ionization potentials at small sizes are higher than the bulk work function and show marked fluctuations as a function of size (Goldstein, 1997).

Enhancement of the nonlinear optical properties by quantum size effects is also of great interest. In nonlinear optical materials, the refractive index can be changed either by carrier injection or by applying electrical fields (Kruis, 1998).

Another feature of nanosized materials is higher chemical reactivity compared to bulk material.

Shapes of nanoparticles also play a role in determining properties such as reactivity and electronic spectra. For example, the position of the plasmon band of metals is sensitive to the aspect ratio (Gogotsi, 2006).

\subsubsection{Applications}

Great impacts of nanoscience on applied technology have resulted in intense interdisciplinary efforts. The dependence of properties on size on the nanoscale has been effectively utilized to control the chemical and physical properties of nanoparticles and to tailor them for specific applications.

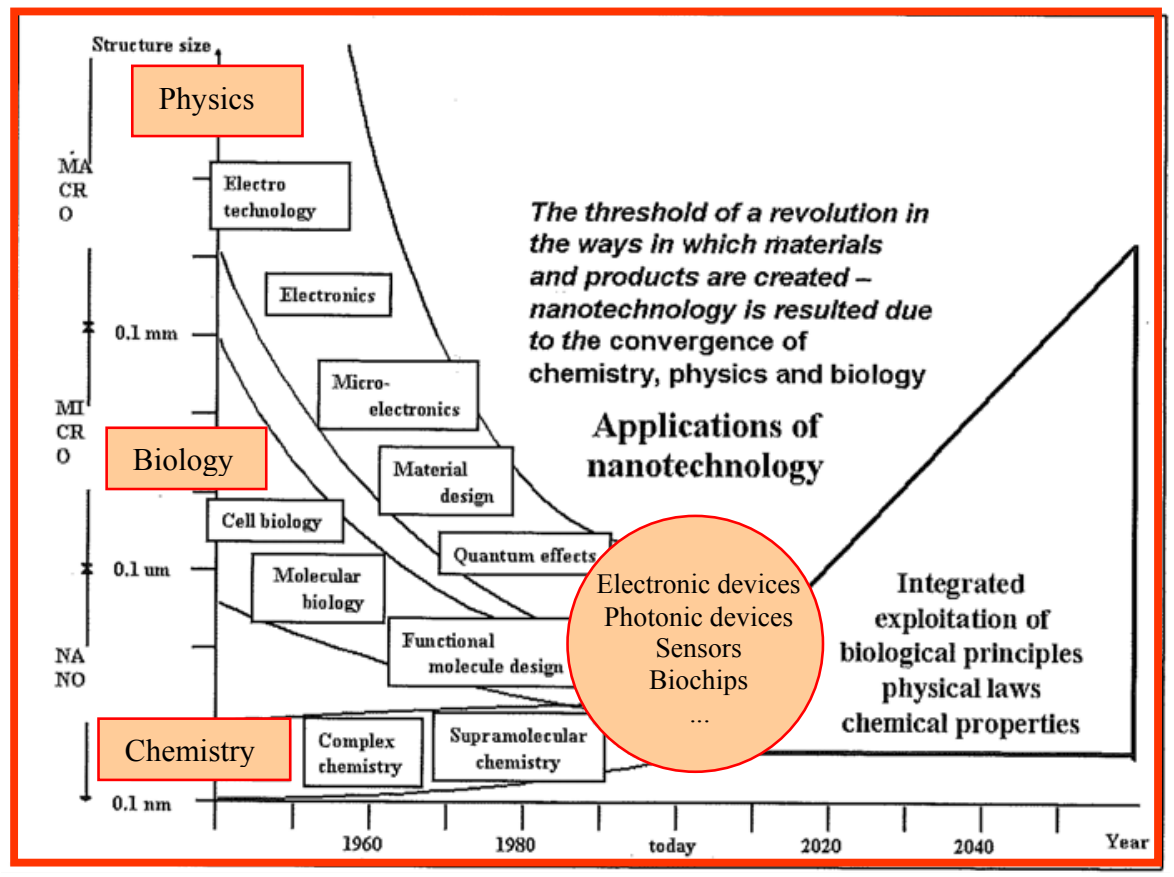

Figure 1.4 Threshold of nanotechnology as basic sciences converge to the nanoscale (from $\mathrm{Lu}$-2004)

For instance biomaterials have found applications in approximately 8000 different kinds of medical devices (Gogotsi, 2006). Drug and gene delivery, molecular motors, such as protein $\mathrm{F}_{1}$-AT phase, monitoring living systems by nanosensors are nanostructure applications in biology.

In recent years, biodegradable polymeric nanoparticles, particularly those coated with hydrophilic polymer such as (poly ethylene glycol) PEG known as long-circulating particles, 
have been used as potential drug delivery devices because of their ability to circulate for a prolonged period of time, target a particular organ as carriers of DNA in gene therapy, and their ability to deliver proteins, peptides and genes (Mohanraj, 2006).

Nanoporous materials as a subset of nanostructured materials possess unique surface, structural, and bulk properties and underline their important uses in various fields such as ion exchange, separation, catalysis, sensor, biological molecular isolation and purifications. They have the ability to absorb and interact with atoms, ions, and molecules on their large interior surfaces and in the nanometer sized pore space (Lu, 2004).

Nanomaterial-based membrane technologies, adsorbents, and catalysts can be utilized to advance water pollution control, groundwater remediation, potable water treatment, and air quality control.

In microworld the ratio of the surface area to the volume of a component is much larger than in conventional-sized devices. This makes friction more important than inertia. Another characteristic of the microworld is that molecular attractions between microscale objects can exceed mechanical restoring forces. Microelectromechanical systems (MEMS) which are based on a mechanical response to an applied electrical signal, or an electrical response resulting from mechanical deformation represent a mature technology and many of the different behaviors observed in the micromechanical world could well apply to the nanoregime, thereby providing a basis for the design of nanomachines which are in the early stages of development. Some examples of nanoelectromechanical systems (NEMS) are actuators and molecular and supramolecular switches. Actuators are devices that convert electrical energy to mechanical energy, or vice versa. It is known that single-walled carbon nanotubes deform when they are electrically charged. This property can be utilized in an actuator. Molecules which display switching behavior might form the basis for information storage and logic circuitry in computers using binary systems. For example cis-to-trans UVlight-induced isomeration of azobenzene can be regarded as a switch behavior (Poole $\mathrm{Jr}$, 2003).

Novel methods of fabricating patterned nanostructures as well as new device concepts are constantly being discovered. Lithography is a method for printing on a smooth surface. It can also refer to photolithography, a microfabrication technique used to make integrated circuits and MEMS.

Quantum dots can be used to produce light emitters of various colors by "band gap tuning" using particle size effects rather than the current complex techniques of synthesizing compound semiconductors (Kruis, 1998). Applications of semiconductors nanostructures as resonant tunneling devices in nanoelectronics have already demonstrated success in multivalued logic and memory circuits. Functional devices based on quantum confinement would be of use in photonic switching and optical communications (Gogotsi, 2006).

The ordered alloys Co-Pt and Fe-Pt are particularly interesting for magnetic recording because of their very high magnetocrystalline anisotropy, making these materials especially useful for practical applications such as magnetic memory devices as well as in biomedicine (Schwarz, 2004). Cobalt, iron, and nickel are interesting as magnetic nanomaterials in ferrofluids, and microwave composite materials (Weisner, 2007).

Using heterojunctions between $\mathrm{p}$-and n-type semiconducting oxides allows developing new sensing mechanisms for gas and humidity sensors. For semiconductor-type gas sensors, the use of materials with a mean particle size well bellow $50 \mathrm{~nm}$ significantly improved the gas sensing properties (Knauth, 2002).

Consolidated nanocomposites and nanostuctures enable production of ultrahigh strength, tough structural materials, strong ductile cements and novel magnets. Dirt-repllent materials, scratch-proof coatings, environmentally friendly fuel cells with highly effective catalysts are 
some utilities of nanostructured materials (Gutsch, 2002). Nanostructured electrode materials could improve the capacity and performance of Li-ion batteries.

Typical capacitances ${ }^{3}$ of nanocrystals are in the range of $10^{-18} \mathrm{~F}$. At such low capacitances, successive charging events are no longer continuous but are discrete. It has been proposed that by using nanocrystals, single-electron devices such as supersensitive electrometers and memory devices could be fabricated (Schwarz, 2004). ). Gold particles are expected to become a material for single-electron devices (Nakaso et al., 2002).

Carbon nanotubes are being used as tips in scanning microscopes and also as efficient field emitters for possible use in display devices. Since SWNTs can be metallic or semiconducting, many applications exploiting their electronic structure can be expected. Very high tensile strength $(\sim 100$ times that of steel) of ropes made of SWCNTs has recently been determined experimentally (Mansoori, 2005).

Silver, gold and copper are essentially used for their color, yellow to red, due to their plasmon resonance located within the visible domain of the electromagnetic spectrum. Palladium, platinum, and ruthenium are largely used for heterogeneous catalysis (Weisner, 2007).

\subsubsection{Health Impacts}

The ability of some nanomaterials to photochemically produce reactive oxygen species (ROS) that may be used to oxidize contaminants or inactivate microorganism may present a risk of toxicity to organism. In fact, not all nanomaterials are benign and some have the ability to cause adverse biological effects at cellular, subcellular, and molecular levels. These potentially harmful effects could be enhanced by the ability of nanoparticles to be taken up and travel through the body, deposit in target organs, penetrate cell membrane, lodge in mitochondria, and trigger injurious responses (Wiesner, 2007).

Since the plethora of nanosized materials may not be biodegradable, rigorous ongoing investigation is required to determine what their behavior will be in various ecosystems, in terms of absorption or desorption, biotic uptake, and accumulation in plants and animals. Biological activity of nanoparticles may be affected by their surface area, shape, oxidant generation, surface charge, solubility, surface functionalization, and chemical composition (Castranova, 2009).

Pulmonary toxicity studies in rats demonstrate that lung exposures to ultrafine or nanoparticles produce greater adverse inflammatory responses compared with larger particles of identical composition at equivalent mass concentrations (Warheit, 2004).

Surface properties and free radical generation by the interaction of particles with cells appear to play important roles in nanoparticle toxicity. The respirability of aerosol particles depends on particle size (Jenkins et al., 2003). Particle deposition in human respiratory tract is shown in Figure 1.5.

Regarding the fullerenes ${ }^{4}$ or buckyballs (C-60 molecules), it is reported that exposure can cause extensive brain damage and alter the behavior of genes in the liver cells of juvenile large mouth bass. It was also reported that inhalation of carbon nanotubes can damage the lungs (Theodore, 2005). Raw single walled carbon nanotubes (SWCNT) contain high levels of metal contamination (30\% by weight) which are often used as catalysts in synthesis processes. Raw SWCNT generate reactive oxygen species in the presence of bronchial epithelial cells (Castranova, 2009).

\footnotetext{
${ }^{3}$ The charging energy $\left(E_{C}\right)$, for example the energy barrier that has to be overcome to transfer a single electron from an initially neutral cluster to a neutral nearest neighboring cluster is dependent on the the inter-particle capacitance (C), as follows from $\mathrm{E}_{\mathrm{C}}=\mathrm{e}^{2} / 2 \mathrm{C}$ where e is the charge of the electron (Simon et al., 1998).

${ }^{4}$ Fullerene is one of the four allotropes of carbon (graphite-nanotube-fullerene-diamond)
} 


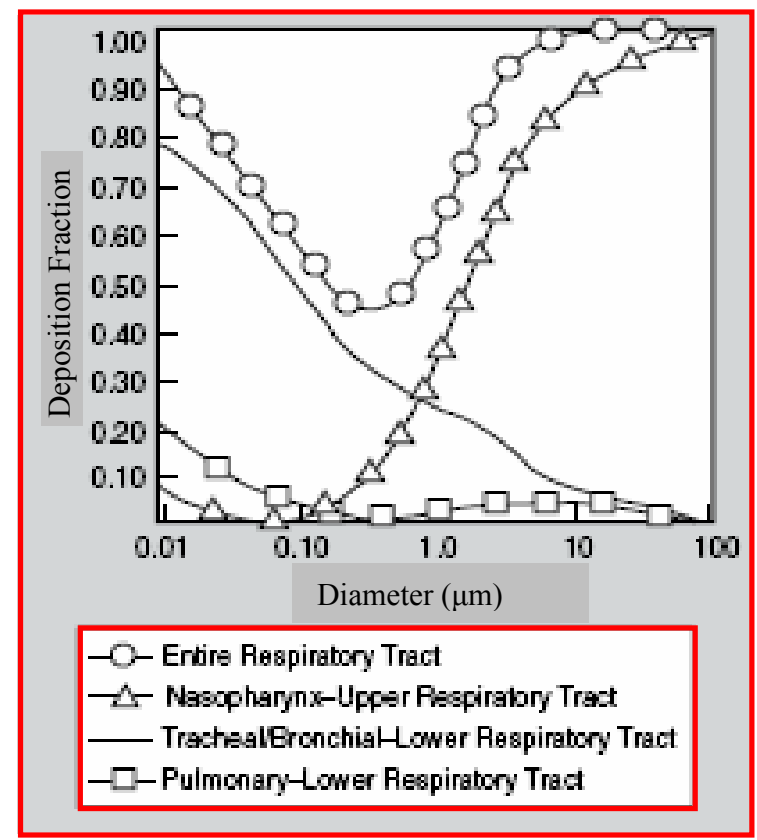

Figure 1.5 The fractional deposition of particles in human respiratory tract $(2,150 \mathrm{~mL}$ tidal volume) (adapted from Jenkins et al., 2003)

High temperature acid treatment effectively removes the contamination from the SWCNT and the purified sample fails to generate oxygen radicals in vitro and is much less toxic to bronchial epithelial cells in culture (Castranova, 2009). Diesel nanoparticles have been found to damage the cardiovascular system in a mouse model (Koch, 2007). Thus far, metal oxide nanoparticles have been shown to generate hydroxyl radicals in vitro and produce acute inflammation in vivo (Castranova, 2009). Few data exist regarding the health risks of dermal or oral exposures to nanomaterials (Warheit, 2004).

\subsubsection{Synthesis}

There are two basic approaches for the synthesis of nanostructured materials, top-down approach, which involves breaking down the bulk material into particles with nanometersized grains, and the bottom-up approach in which individual atoms or molecules are put together to form the nanoparticles.

The fabrication method is of significant importance because it determines the particle composition, structure, size and size distribution. All particle synthesis techniques fall into one of the three categories: vapor phase, solution precipitation, and solid-state processes. Mechanical alloying, high energy ball milling, equal channel angular pressing, high pressure torsion, and accumulative roll bonding are mechanical processes (Koch, 2007). The disadvantages of mechanical attrition processes for nanoparticle synthesis include impurity pick up, lack of control on the particle size distribution, and ability to tailor precisely the shape and size of particles in the 10 to $30 \mathrm{~nm}$ range, as well as the surface characteristics (Gogotsi, 2006).

Particle synthesis in solution or mixture involves chemical reaction between the reagents (e. g. reduction of metal ions in solution) which leads to the formation of nuclei followed by the growth of particles. Homogenous nucleation does not involve foreign species as nucleating aids. Heterogeneous nucleation, however allows formation of nuclei on foreign species. Factors such as supersaturation, nucleation and growth rates, colloidal stability, and 
recrystallization and aging processes have effects on the particle size and microstructure. Supersaturation generally shows predominant influence on the morphology of precipitates. At low supersaturation, the particles are small, compact and well-formed, and the shape depends on crystal structure and surface energies. At high supersaturation, large and dendritic particles form. At even higher supersaturation, smaller but compacted agglomerates form (Koch, 2007).

Particles synthesized by precipitation and the sol-gel method may require subsequent heat treatment for dehydration, removal of organics, and controlled crystallization to form particles with desirable structure and crystallite size. A detailed review on nanochemistry of metals has been written by Sergeev (2001).

Most synthesis methods of nanoparticles in the gas phase are based on homogeneous nucleation in the gas phase and subsequent condensation and coagulation. The attractive forces between molecules in a vapor lead to the formation of molecular clusters which are unstable and continuously disintegrate. If the vapor is supersaturated, the number concentration of the clusters increase as well as the collisions which result in clusters exceeding a threshold size (Kelvin diameter ${ }^{5}$ ). Clusters having this critical diameter are stable and grow by condensation to form particles. The rate of growth depends on the saturation ratio, particle size and particle size relative to the gas mean free path (Hinds, 1999). For particle larger than the gas mean free path, growth depends on the rate of diffusion of molecules to the droplet surface (Hinds, 1999).

The small particles dispersed in the fluid move randomly due to Brownian motion and collide with each other along their trajectories. Assuming strong adhesive forces, characteristic for small particles, or chemical bonds, these collisions result in coagulation (Gutsch, 2002).

Smoluchoweski derived an expression for the collision frequency of spherical particles in Brownian motion (Hinds, 1999). In this theory, the particle collision rate is assumed to be diffusion limited process, and collision is followed by instantaneous coalescence. Subsequently, Fuchs extended Smoluchowski's theory to the free molecular regime where the particle sizes are smaller than the mean free path of the gas (Fuchs, 1989). The widely applied synthesis methods of nanoparticles in the gas phase, using solid precursors, are inert gas condensation, ion sputtering, pulsed laser ablation and spark discharge generation (Swihart et.al., 2003).

Gas-phase synthesis has the advantage of high purity due to the absence of liquid solvents and the higher thermal stability of gases with respect to liquids makes the approach very flexible. Gas phase synthesis usually delivers crystalline particles, which is desirable for many applications. Another important feature is the fact that aerosol routes for particle production are continuous, whereas liquid processes are usually batch processes.

\subsection{Thesis Organization}

Motivated by the above-mentioned general advantages of particle synthesis in the gas phase, we focused on the spark discharge technique which is simple, compact, and a versatile method to produce nanoparticles from conductive materials. Besides, the synthesis is performed at atmospheric pressure which is quite economic in comparison with vacuum methods and has the potential of being scaled up.

The objectives of this work were to investigate on:

\footnotetext{
${ }^{5}$ The diameter of the droplet that will neither grow nor evaporate at saturation pressure on the particle surface
} 
$\checkmark$ Working principles in the spark discharge generator

$\checkmark$ Features of nanoparticles produced by the spark discharge technique

$\checkmark$ The ability of the spark discharge to generate internally mixed nanoparticles from miscible and immiscible metals

$\checkmark$ Particle formation by the spark discharge from theoretical points of view

Each of these topics is covered in a separate chapter.

In spite of the fact that spark discharge has been widely used to produce nanoparticles for basic studies, there is a lack of a systematic study on this technique in previous work. Thus in the next chapter the performance of the spark discharge generator at various operating conditions is studied and our observations are discussed qualitatively.

In chapter three, emphasis is placed on the synthesis and characterization of various types of nanoparticles. Bottle-necks encountered in practice are explained and discussed. To investigate the applicability of the particles, a preliminary study on the hydrogen storage properties of $\mathrm{Mg}$ and $\mathrm{Pd}$ nanoparticles is reported in this chapter.

The spark discharge method has previously been utilized to produce metallic particles, metal oxide, and carbon nanoparticles. The ability of this method to generate mixed metallic and composite nanoparticles is expected to open a new field of applications for this technique. Therefore, we investigated on the mixing ability of spark discharge process for miscible systems in chapter four. Since the main mechanism of particle formation in spark discharge is evaporation-condensation, systems with large and small differences in the evaporation rates are studied. Furthermore, the mixing behavior of spark at different operating conditions is investigated in this chapter.

Recent studies show that the heat of formation of alloy nanoparticles is dependent not only on composition but also on size (Xiao et al., 2006) and some immiscible systems exhibit alloy formation as the particle sizes reduce to a critical size (Ouyang et al., 2006). Furthermore, the nonequilibrium condition ${ }^{6}$ may lead to an enhanced defect density and solid solubility (Dirks, 1985). Thus in chapter five alloy formation from immiscible systems is studied.

To produce mixed particles, various types of electrodes (a pair of single element electrodes, compacted, sintered, and alloyed electrodes) with different degrees of premixing have been applied (Figure 1.6).

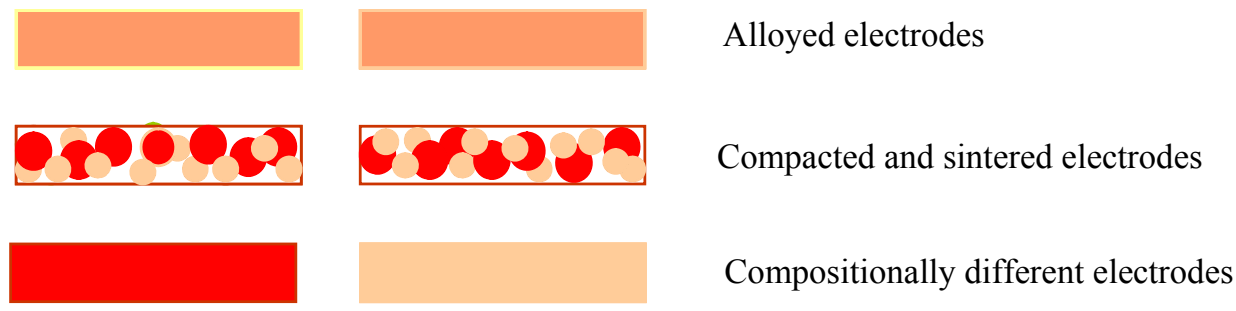

Figure 1.6 Different level of premixing in the applied electrodes

In generation of bimetallic nanoparticles, it is essential to distinguish a physical mixture of compositionally different nanoparticles from internally mixed particles (Figure 1.7). So characterization methods are of crucial importance. Thus in chapter four and five various techniques are utilized to characterize the particles.

Due to the difficulty of incorporating the aerosol dynamics in the fluid dynamic, mass and heat transfer regimes, accurate prediction of primary particle size distributions and their

\footnotetext{
${ }^{6}$ The cooling rate in a welding arc is about $10^{7} \mathrm{~K} / \mathrm{s}$ (Jenkins, 2003), thus in a microsecond the vapor is cooled about $10 \mathrm{~K}$.
} 
aggregates in the gas phase is not straight forward especially noting that the coalescence rate is strongly dependent on the local gas temperature. Nevertheless, aerosol dynamics can be used to explain the trends in experimental particle size data (Bandyopadhyaya et al., 2003).

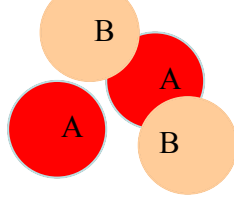

(a) Physical mixture of nanoparticles

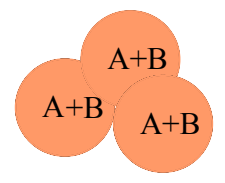

(b) Internally mixed nanoparticles

Figure 1.7 Different level of mixing in nanoparticles

Thus in chapter six, on the basis of a model derived by Lehtinen et al. (1996), the collision and coalescence characteristic times for some examples are calculated and the predicted primary particle sizes are compared with the experimental results. The key parameters causing close primary particle sizes synthesized from various materials by spark discharge are discussed.

\section{References}

Bandyopadhyaya Rajdip, Anshuman A. Lall, Sheldon K. Friedlander, Aerosol Dynamics and the Synthesis of Fine Solid Particles, Powder Technology, Volume 139, 2004, pp. 193-199

Castranova V., The Nanotoxicology Research Program in NIOSH, Journal of Nanoparticle Research, 2009, DOI 10.1007/s11051-008-9421

Dirks A. G., J. J. van den Broek, Metastable Solid Solution in Vapour Depositd Cu-Cr, Cu-Mo, and Cu-W Thin Films, J. Vac. Sci. Technol. A, Volume 3, Number 6, 1985, pp. 2618-2622

Edelstein A S, Cammarata RC, Nanomaterials: Synthesis, Properties and Applications, Institute of Physics Publishing Bristol and Philadelphia, 1996

Feldheim Daniel L., Colby A. Foss. Jr., Metal Nanoparticles Synthesis, Characterization, and Application, Marcel Dekker, Inc., 2002

Fuchs N. A., the Mechanics of Aerosols, Dover Publications, New York, 1989

Gogotsi Yury, Nanomaterial Handbook, Taylor\& Francis group, LLC., 2006

Goldstein Avery N., Handbook of Nanophase Materials, Marcel Dekker, 1997

Granqvist C. G., R. A. Buhrman, Ultrafine Metal Particles, Journal of Applied Physics, Volume 47, Number 5, 1976, pp. 2200-2219

Gutsch A., M. Kramer, G. Micael, H. Muhlenweg, M. Pridohl, G. Zimmermann, Gas-Phase Production of Nanoparticles, KONA, Number 20, 2002, pp. 24-37

Hinds C. William, Aerosol Technology Properties, Behavior, and Measurement of Airborne Particles, John Wiley \& Sons, 1999

Jenkins Niel T., Thomas W. Eagar, Submicron Particle Chemistry: Vapour Condensation Analogous to Liquid Solidification, JOM Journal of the Minerals, Metals and Materials Society, Volume 55, Number 6, 2003, pp. 44-47 
Knauth Philippe, Schoonman Joop, Nanocrystalline Metals and Oxides, Kluwer Academic Publishers, 2002

Koch Carl C., Nanostructured Materials, William Andrew, 2007

Kruis F. Einar, Heinz Fissan, Aaron Peled, Synthesis of Nanoparticles in the Gas Phase for Electronic, Optical and Magnetic Application- a Review, Journal of Aerosol Science, Volume 29, Number 5/6, 1998, pp. 511-535

Lehtinen Kari E. J., Robert S. Windeler, Sheldon K. Friedlander, Prediction of Nanoparticle Size and Onset of Dendrite Formation Using the Method of Characteristic Times, Journal of Aerosol Science, Volume 27, Number 6, 1996, pp. 883-896

Lu G. Q., Zhao X. S., Nanoporous Materials Science and Engineering, Imperial College Press, 2004

Mansoori Ali G., Principle of Nanotechnology Molecular-Based Study of Condensed Matter in Small Systems, World Scientific, 2005

Mohanraj V J, Chen Y, Nanoparticles-A Review, Tropical Journal of Pharmaceutical Research, 2006, Volume 5, Number 1, pp. 561-573

Nakaso Koichi, Manabu Shimada, Kikuo Okuyama, Knut Deppert, Evaluation of the Change in the Morphology of Gold Nanoparticles During Sintering, Journal of Aerosol Science, Volume 33, 2002, pp. 1061-1074

Ouyang G., X. Tan , C. X. Wang, G. W. Yang, Charge- Induced Transition between Miscible and Immiscible in Nanometer-Sized Alloying Particles, Chemical Physics Letters, Volume 423, 2006, pp. 143-146

Poole Jr., Charles P., Owens Frank J., Introduction to Nanotechnology, WILEY, 2003

Rotello Vincent, Nanoparticles Building Blocks for Nanotechnology, New York, Kluwer Academic/Plenum, 2004

Schwarz James A., Contescu Cristian I., Putyera Karol, Dekker Encyclopedia of Nanoscience and Nanotechnology, Taylor \& Francis, 2004

Sergeev G B, Nanochemistry of metals, Russian Chemical Reviews, 2001, Volume 70, Number 10, pp. 809-825

Simon U., R. Flesch, H. Wiggers, G. Schon, G. Schmid, Chemical Tailoring of the Charging Energy in Metal Cluster Arrangements by Use of Bifunctional Spacer Molecules, Journal of Materials Chemistry, Volume 8, Number 3, 1998, pp. 517-518

Swihart Mark T., Vapor-Phase Synthesis of Nanoparticles, Current Opinion in Colloid and Interface Science, Volume 8, 2003, pp. 127-133

Theodorre Louis, Kunz Robert G., Nanotechnology Environmental Implications and Solutions, WILEY, 2005

Warheit David B., Materials Today, February 2004, pp. 32-35

Waseda Y., A. Muramatsu, Morphology Control of Materials and Nanoparticles Advanced Material Processing and Characterization, Springer, 2004

Wiesner Mark R., Bottero Jean-Yves, Environmental Nanotechnology, McGrawHill, 2007

Wu M. K., R. S. Windeler, C. K. R. Steiner, T. Börs, S. K. Friedlander, Controlled Synthesis of Nanosized Particles by Aerosol Processes, Aerosol Science and Technology, Volume 19, 1993, pp. 527-548

Xiao S., W. Hu, W. Luo, Y. Wu, X. Li, H. Deng, Size Effect on Alloying Ability and Phase Stability of Immiscible Bimetallic Nanoparticles, The European Physical Journal B, Volume 54, 2006, pp. 479-484

Yacaman M. Jose, J. A. Ascencio, H. B. Liu, J. Gardea-Torresdey, Structure Shape and Stability of Nanometric Sized Particles, Journal of Vacuum Science and Technology, Volume 19 B, Number 4, 2001, pp. 1091-1103 


\section{Chapter 2}

\section{Spark Discharge Generator}

\subsection{Introduction}

Aerosol production from electrical discharges has already been observed in 1907 (Hemsalech et al.). A nanoparticle generator based on this principle was developed by Schwyn et al. in 1988. The method can be applied to any conductive material including semiconductors. It produces particles very similar to laser ablation (Ullmann et al., 2002), but is simpler and in contrast to this method it has the potential of being scaled up to produce larger quantities. No crucible is required, there is no melting point limitation and the method does not require expensive precursors. The same set-up can be used for production of different nanoparticulate materials. This technique has been widely used but it has insufficiently been studied systematically. The objective of the study presented in this chapter is to gain a better understanding of the method in order to obtain a better judgment of its potential.

\subsubsection{Previous Work}

In the work by Schwyn et al.(1988), the combination of spark discharge and electrical classification led to the generation of monodisperse particles down to $1.3 \mathrm{~nm}$ diameter in a concentration of $3 \times 10^{5} \mathrm{~cm}^{-3}$. Dilution of the aerosol by injecting the carrier gas through a nozzle at the region of particle formation avoided particle agglomeration.

A study on the physical properties and the chemical composition of spark-generated aerosol and the corresponding erosion craters was undertaken by Watters et al. (1989). They stated that in order to avoid chemical biases due to melting point differences, the time that electrode materials exist in the molten liquid state should be minimized by a rapid vaporization. They showed that the size distributions of particles generated by the spark fell into at least two groups. The first group consisted of spheres $0.01 \mu \mathrm{m}$ in diameter and the second group consisted of spheres up to several micrometers in diameter. It was suggested that the spark frequency and current might play an important role in determining the relative population of these groups.

A study by Helsper et al. (1991) on soot-like particles produced by the spark discharge between two graphite electrodes in argon showed that the particle mass flow varied from 20 $\mu \mathrm{g} \mathrm{h}^{-1}$ to $7 \mathrm{mg} \mathrm{h}^{-1}$ by changing the spark frequency. The mass production exhibited a linear relationship with the spark frequency. Primary particle size was in the range of $5 \mathrm{~nm}$, forming agglomerates between 50 and $200 \mathrm{~nm}$. In their study the electrode consumption was automatically compensated by a belt driven mechanism, which gradually closed the gap sufficiently to attain a constant breakdown voltage hence a constant operation.

Regarding the effect of carrier gas type on the spark, Mäkelä et al. (1992) reported that particle size and stability of concentration mainly depended on the carrier gas, and nitrogen 
as a carrier gas led to a much higher output concentration stability compared to air. By varying the capacitance from 0.4 to $20 \mu \mathrm{F}$, the mean diameter was shifted from 10 to $15 \mathrm{~nm}$. To measure the original ions produced by the spark generator ${ }^{1}$, they detected the carrier gas ions produced by the aerosol charger. They stated that most of the particles of 2-4 nm were not initially charged in the spark generator.

A reactive spark erosion technique was utilized by Hsu et al. (1994) to produce titanium carbide particles in a carbonic dielectric liquid (pentane) with titanium electrodes. The particles showed a bimodal size distribution (5-50 $\mathrm{nm}$ and 5-20 $\mu \mathrm{m})$.

In a study performed by Ullmann et al. (2002), a comparison was made between the aerosols generated by spark discharge technique and laser ablation method. It was demonstrated that for silica, alumina, titania, iron oxide, tungsten oxide, niobium oxide, carbon, and gold generated by laser ablation, the primary particle size ranged between 4.9 and $13 \mathrm{~nm}$ and for spark discharge generated particles, the primary particle size was in the same range with even less variation between the materials.

In another study by Horvath et al. (2003), carbon nanoparticles were produced in a low voltage spark generator using a Tesla transformer. The capacitor was charged to voltages of about $100 \mathrm{~V}$ for the discharge. The decoupling of charge, voltage and frequency enabled choosing the operating conditions. A very stable and continuous operation for tens of hours was reported.

In production of carbon using a commercial spark generator (PALAS), Evans et al. (2003) showed that an increase in the spark frequency enhanced the total particle number production, the agglomerate modal diameter and the aerosol mass concentration. Structures of the agglomerates were studied using the fractal geometry.

Seipenbusch et al. (2003) studied the kinetics of the primary particle growth and restructuring of the agglomerates of $\mathrm{Ni}$ and $\mathrm{Pt}$ generated by the spark discharge technique. They observed no dependence of the particle diameter on capacitance (varying from 20 to $100 \mathrm{nF}$ ) and nitrogen flow rates (varying from 1 to $5 \mathrm{~L} / \mathrm{min}$ ). They observed an increase in the aerosol mass concentration roughly by a factor of 3 while the capacitance was changed by a factor of 5.

Utilizing the commercial spark generator (PALAS), Roth et al. (2004) produced carbon, metal, metal oxide particles with lognormal size distributions and agglomerate modal diameters in the range of $18-150 \mathrm{~nm}$ and geometric standard deviation of about 1.5. Each material used for the generation of nanoparticles was observed to be associated with a certain morphology (for example spherical, nonspherical particles, and chain agglomerates) and surface area. They reported that each discharge consisted of 10 single oscillating sparks of 4 $\mu$ duration and different polarity.

Copper oxide aerosol particles were generated from copper electrodes by Kim et al. (2005). The inter-electrode gap and the gas flow rate were used to control the particle mass density. Hinot et al. (2007) used spark discharge method to investigate the effectiveness of the contact between Pt nanoparticles and soot in lowering the catalytic soot oxidation temperature. For this purpose they studied Pt-doped soot (applying one electrode of Pt and the other electrode

\footnotetext{
${ }^{1}$ In the size range about $2 \mathrm{~nm}$ the ions from the carrier gas generated by aerosol charger may distort the electrical measurement of generated distribution (Mäkelä et al.,1992).
} 
of carbon) and separately generated soot and Pt and showed that the distance between soot and Pt particles plays a key role.

Oh et al., 2007 produced titania nanoparticles from titanium electrodes via spark discharge method under an air atmosphere. The size and concentration of the particles were controlled via spark frequency, capacitance, gap distance, and gas flow rate. The aggregated particles were amorphous and composed of necked primary particles of few nanometer sizes.

The scattered information obtained from the previous work on the spark discharge generator (SDG), hardly reveals features of general validity. This task is dealt with in the present chapter. To begin with, an introduction to the electrical breakdown of gases and formation of plasma is given below.

\subsubsection{Breakdown in Gas}

When a low voltage is applied on a gaseous dielectric, a small current flows between the electrodes and the insulation retains its electrical properties. If the applied voltage is high, the current flowing through the insulation increases sharply and electrical breakdown occurs. The breakdown voltage is the maximum voltage applied to the insulation at the moment of breakdown. The electrical discharges in gases can be of non-sustaining or self-sustaining types. Spark discharge is a transition of a non-sustaining to a self-sustaining discharge (Naidu, 1995). During the short breakdown period (usually 0.01-100 $\mu \mathrm{s}$ ) the non-conducting gas becomes conductive and generates plasma. Plasma is an ionized gas comprised of molecules, atoms, ions, electrons, and photons which is electrically neutral on a macroscopic scale. Depending on the energy of the plasma, the degree of ionization may be so high that virtually no neutral particles are left. In thermal plasma, local thermodynamic equilibrium (LTE) exists and $\mathrm{T}_{\text {electron }}=\mathrm{T}_{\text {particle }}$ (for example $>10^{4} \mathrm{~K}$ ), in nonthermal plasma there is a strong deviation from equilibrium and $\mathrm{T}_{\text {particle }}<<\mathrm{T}_{\text {electron }}$ (typically $\mathrm{T}_{\text {electron }} \sim 10^{4} \mathrm{~K}$ ).

Micro-wave $(\mathrm{GHz})$, radio frequency inductively coupled plasma (rf-ICP,MHz) and dc arc torches, at atmospheric pressure lead to thermal plasma, while dc to $100 \mathrm{kHz}$ ac atmospheric pressure electrical discharges create nonthermal plasmas (Borra, 2006). All plasmas are initiated by an electron avalanche for example, multiplication of some primary electrons in cascade ionization (Figure 2.1.a). This necessitates the presence of a free electron in addition to a high electric field to accelerate electrons up to a kinetic energy higher than the ionization potential of the gas molecules (Borra, 2006). The velocity of electron avalanches is about $10^{5}$ $\mathrm{ms}^{-1}$, crossing a gap of $1 \mathrm{~mm}$ distance in about $10^{-8} \mathrm{~s}$ (Soldera et al., 2004). In ionization process, electrons and ions produced from the neutral atoms migrate towards the anode and cathode, respectively, resulting in high currents.

Anode

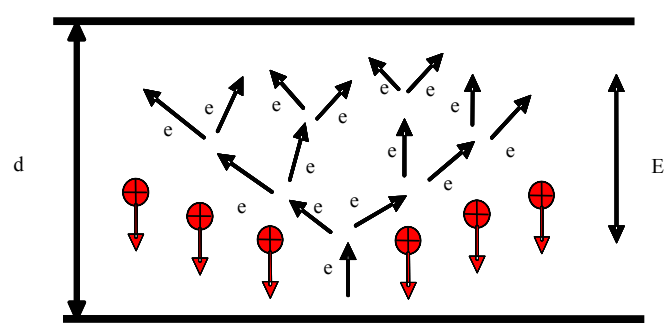

Cathode (a)
Anode

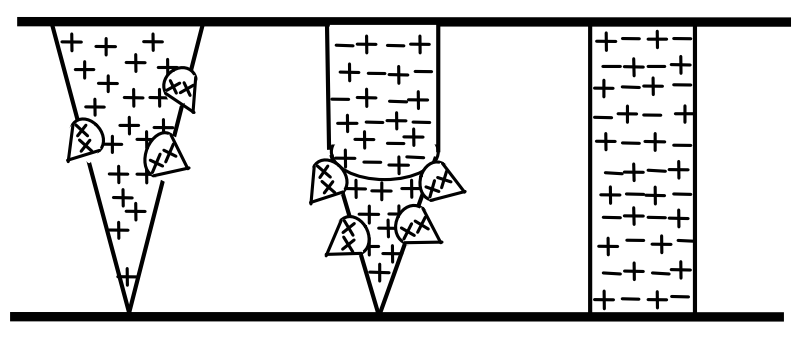

Cathode (b)

Figure 2.1 Townsend breakdown gap (reproduced from Fridman, 2004) (a), cathode directed streamer (reproduced from Naidu, 1995) (b) 
Two types of theories are known to explain the breakdown mechanism, Townsend theory and Streamer theory. According to the Townsend coefficient $(\alpha)$ definition, each primary electron generated near the cathode produces $\exp (\alpha \mathrm{d})-1$ positive ions in the gap ( $\mathrm{d}$ is the gap distance). The positive ions produced move towards the cathode and altogether eliminate $\gamma[\exp (\alpha \mathrm{d})-1]$ electrons from the cathode in the process of secondary electron emission. The secondary electron emission coefficient $(\gamma)$ characterizing the probability of generation of a secondary electron on the cathode by an ion impact, depends on the cathode material, surface state, gas type, and reduced electric field (E/P where $\mathrm{E}$ is the electric field and $\mathrm{P}$ the pressure). The current in the gap given by the Townsend formula is (Fridman, 2004):

$$
i=\frac{i_{0} \exp (\alpha d)}{1-\gamma[\exp (\alpha d)-1]}
$$

When the electric field becomes sufficiently large, the denominator in equation (2.1) goes to zero and breakdown occurs. By neglecting the second term in the bracket which is normally small compared to the first term, Townsend's breakdown criterion can be written as:

$$
\gamma \exp (\alpha d)=1
$$

In electronegative gases, attachment of electrons to neutral atoms or molecules forming negative ions, results in removing electrons and thus breakdown at high voltages. Oxygen is such a gas. To include ionization and attachment, the Townsend current growth equation must be modified for such gases (Naidu, 1995).

Paschen's Law reflects the Townsend breakdown mechanism in gases (equation 2.3). The significant parameter is P.d, and suitable parameters (A, B) are chosen by fitting to empirical data.

$$
V=\frac{B P d}{\ln (P d A)-\ln \left[\ln \left(\frac{1}{\gamma}+1\right)\right]}
$$

The Townsend mechanism explains the breakdown phenomena only at low pressures, corresponding to P.d (gas pressure $\times$ gap distance) values of 1000 torr-cm and below. The dependence of breakdown voltage on the gas pressure and geometry of the gap could not be well explained by Townsend mechanism. It predicts breakdown time lags in the order of $10^{-5} \mathrm{~s}$, while in practice breakdown is observed to take place after times as short as $10^{-8} \mathrm{~s}$. Moreover discharges were found to be filamentary and irregular. Due to these drawbacks of Townsend's theory, Streamer theory was proposed. According to this theory, a single electron builds up an avalanche that crosses the gap. Since electrons move fast compared to positive ions, by the time that they reach to the anode the positive ions are still at their original positions forming a positive space charge at the anode (Figure 2.1.b). This enhances the field, and photoionization in the space charge region produces a few electrons causing secondary avalanches. This leads to a further increase in the space charge. Electrons of the secondary avalanches are pulled by the strong electric field into the positively charged tail of the primary avalanche creating a streamer propagating fast between the electrodes (Fridman, 2004). This theory can explain the observed filamentary, crooked channels, branching of the spark channels and breakdown in a high pressure gas across a long gap.

The electrical regimes in discharges at ambient pressure depend on the nature of the electrodes, the geometry, the excitation mode (pulsed, dc, or ac), and the gas (Borra, 2006). 


\subsection{Spark Discharge Generator}

The process of spark discharge is initiated by gas breakdown and formation of a conducting channel, dissociation and ionization of the gas molecules within a very short time. The plasma channel then thermally expands and a shock wave is formed.

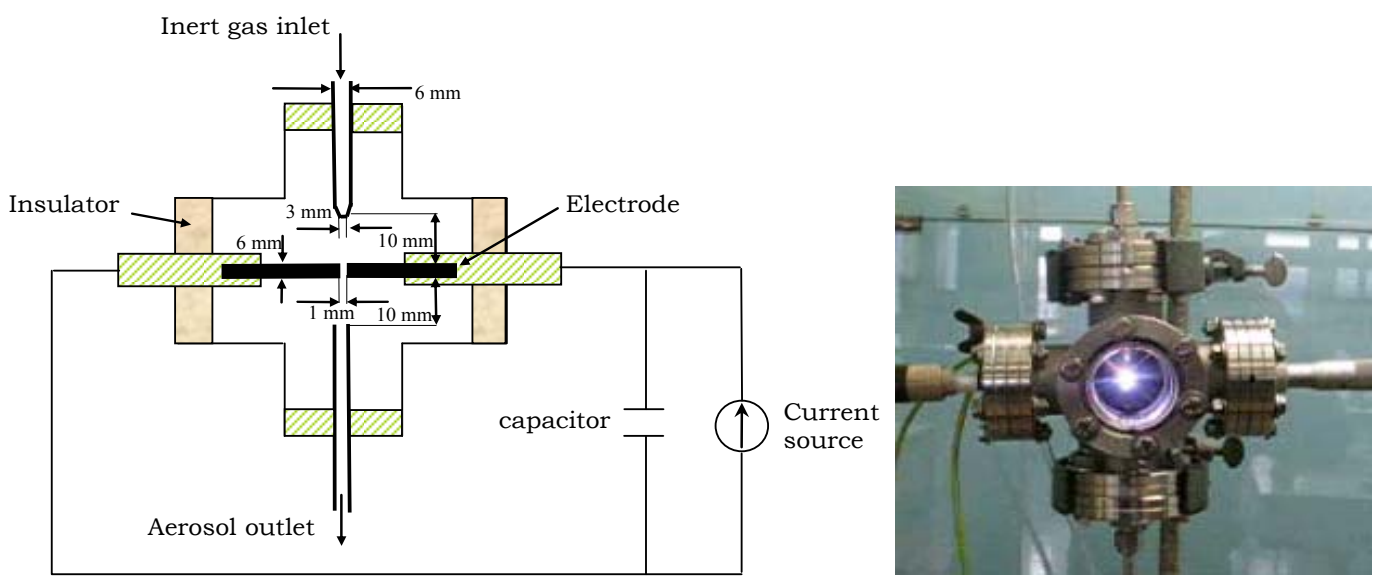

Figure 2.2 Spark discharge generator

In the final stage charge carriers recombine, the plasma cools and the shock wave is attenuated to a sound wave (Reinmann et al., 1997). The principal mechanism of spark generated acoustic waves in solids is the exposure of their surfaces to a strong divergent shock wave produced by expansion of the discharge plasma (Krylovt, 1992).

The breakdown phase is characterized by very high peak values of voltage and current, and an extremely short duration. At a very early stage a cylindrical channel develops (for example $40 \mu \mathrm{m}$ diameter) together with a rapid temperature rise to tens of thousand $\mathrm{K}$ (typically 20000 $\mathrm{K}$, Reinmann et al., 1997). The pressure jumps up, causing the emission of an intense shock wave. Thus, the temperature drops as a function of time and the potential energy (dissociation and ionization) is reconverted to thermal energy. The arc and the glow discharge must always be preceded by a breakdown phase which provides the conductive path between the electrodes necessary to start these discharges. As the arc requires a hot cathode spot, there is also severe erosion (evaporation) of the cathode material. Due to continuous energy losses the equilibrium kernel gas temperature will be limited to typically $6000 \mathrm{~K}$ (Maly, 1979). In glow discharge overall losses are higher than in the arc and the equilibrium kernel gas temperature will decrease (for example $3000 \mathrm{~K}$ ) and the electrode erosion is practically negligible (Maly, 1979). An increase in breakdown energy does not manifest itself in higher kernel temperatures, instead the channel diameter increases, causing a larger activated gas volume (Maly, 1979).

Electrode material is evaporated in the vicinity of the spark. This is followed by rapid cooling initially governed by adiabatic expansion and radiation and below the evaporation temperature dominated by thermal conduction. Because the vapour cloud is small compared to other evaporation-condensation processes, the cooling below the boiling point is relatively fast, and high a concentration of very small particles forms.

Figure 2.2 shows the spark generator that was built in our laboratory for the present project (Appendix A). It consists of a chamber about $300 \mathrm{~cm}^{3}$ in volume, in which two opposing cylindrical electrodes are mounted at an adjustable distance. The electrodes are $3-6.35 \mathrm{~mm}$ in diameter (supplied by Goodfellow, Sigma Aldrich), and the gap between them is adjustable from zero to a few millimeters. They are connected to a high voltage power supply (TECHNIX), and parallel to a variable capacitor with a maximum capacitance of $20 \mathrm{nF}$. A 
high voltage probe connected to an oscilloscope (TEKTRONIX) was used to record the voltage across the spark gap during the discharge. The power supply delivers a constant current, periodically recharging the capacitor after discharge has occurred at the breakdown voltage.

\subsubsection{Electrical Circuit}

The capacitance box consists of three copper bars into them holes are drilled for mounting the capacitors. In order to prevent any stray discharges from the capacitors towards the high voltage source, ten Silicon Diode IN4007 (or Diode IN4007) provided by RS are used. Figure 2.3 depicts the capacitor box as well as the electrical circuit diagram. The required capacitance is obtained by connecting the capacitors in parallel. In this way, at the same voltage, the total capacitance is the sum of connected capacitances $\left(\mathrm{C}_{\mathrm{T}}=\mathrm{C}_{1}+\mathrm{C}_{2} \ldots+\mathrm{C}_{\mathrm{N}}\right)$.

The 1000:1 voltage divider is obtained by resistors of $100 \mathrm{M} \Omega / 100 \mathrm{k} \Omega$. For safety reason the circuit can work only when the box is completely closed. In order to dissipate the remaining charges on the capacitors in the case that the box cover is opened, additional resistor $(100 \mathrm{k} \Omega)$ is used.

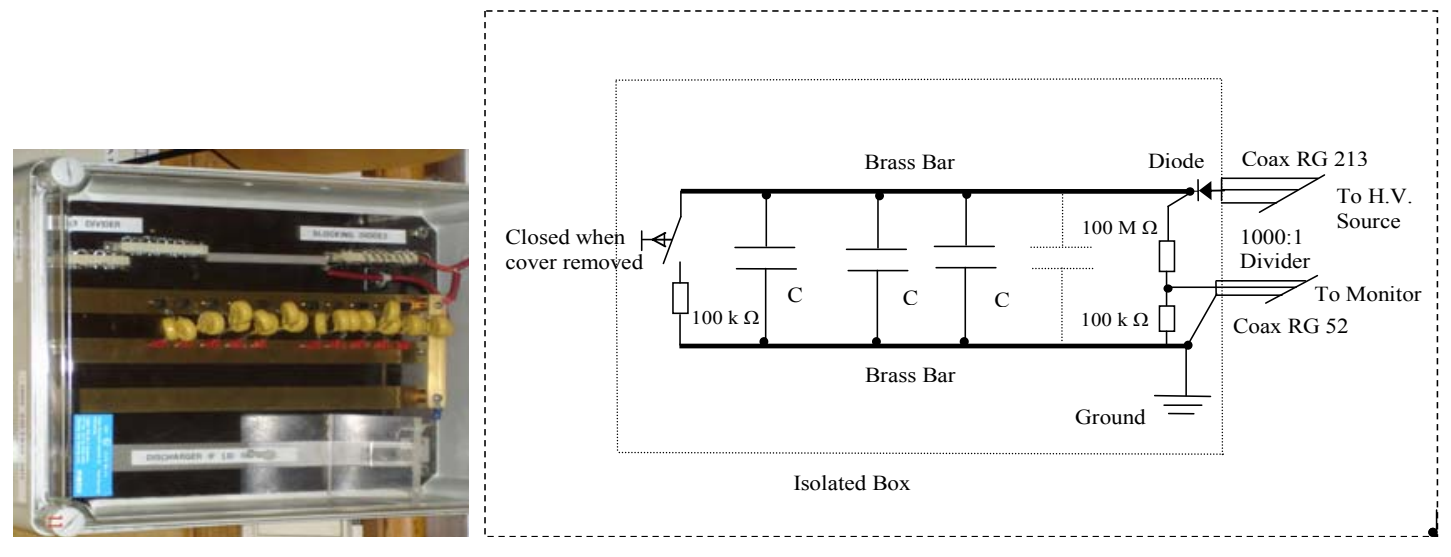

Figure 2.3 Capacitance box and the electrical circuit

\subsubsection{Spark Frequency}

The energy dissipated in every discharge is given by the capacitance $\mathrm{C}$ and the discharge voltage $V_{d}$ which in turn depends on the electrode gap distance and the pressure. In our measurements the values of the spark energy $E$ and the charge $\left(\mathrm{CV}_{\mathrm{d}}\right)$ reached a maximum of $250 \mathrm{~mJ}$ and $6.25 \times 10^{14}$ electrons/spark, respectively. The former is given by:

$$
E=\frac{1}{2} C V_{d}^{2}
$$

At a fixed inter-electrode gap and fixed capacitance, the repetition frequency is determined by the constant current I charging the capacitor and the voltage $V_{d}$ at which the discharge occurs according to:

$$
I_{c}=C \frac{d V_{d}}{d t}
$$

$\mathrm{V}_{\mathrm{d}}$ is somewhat higher than the breakdown voltage $\mathrm{V}_{\mathrm{b}}$, because the discharge conditions require some time to develop. $\mathrm{V}_{\mathrm{b}}$ is the voltage at which gas breakdown would occur at very slow increase of the voltage. In the general case $\mathrm{V}_{\mathrm{d}}=\mathrm{V}_{\mathrm{b}}+\mathrm{V}_{0}$ where $\mathrm{V}_{0}$ is called overvoltage. Thus the repetition frequency is given by: 


$$
f=\frac{I}{C V_{d}}
$$

The values of $\mathrm{V}_{0}$ are rather unpredictable. This is why we measure the frequency rather than calculating it. The actual time the gas breakdown (formation of conducting channel) requires is sensitive to the electric field and thus $\mathrm{V}_{\mathrm{d}}$ (Sher et al., 1992). The ionisation process is also affected by gas temperature and pressure, the field configuration, the nature of electrode surface (Naidu et al., 1995), and the gas composition including impurities.

Typical plots of the gap voltage vs. time are seen in Figure 2.4. From this figure the spark frequency can be derived. The rising ramp indicates charging of the capacitor with about $\mathrm{dV} / \mathrm{dt}=2 \times 10^{4} \mathrm{Vs}^{-1}$. Considering the capacitance equation (2.5), and a capacitance of $\mathrm{C}=2 \mathrm{nF}$, this is in agreement with the charging current of $0.04 \mathrm{~mA}$. The rapid voltage decrease that follows is due to formation of the spark. The spark gap then becomes conducting and the discharge characteristic is of a weakly damped oscillation, as seen on the scale of Figure 2.4.b.

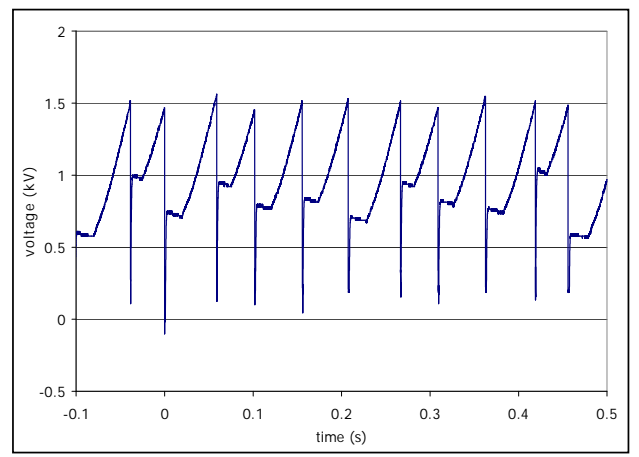

(a)

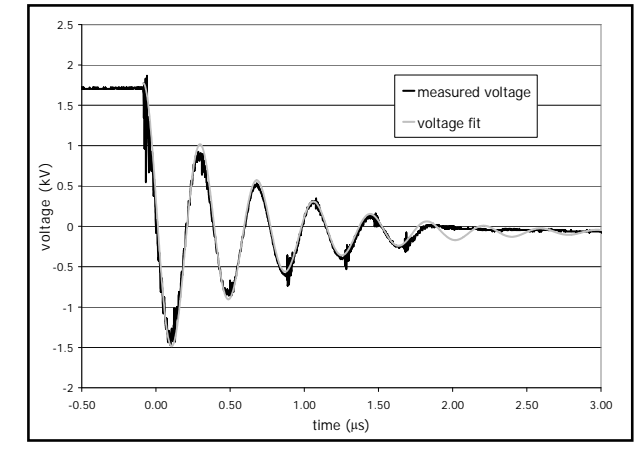

(b)

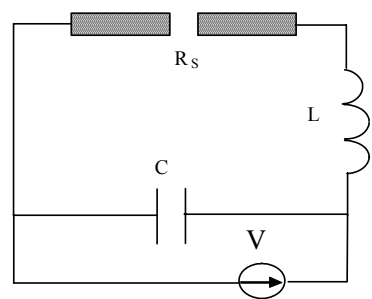

(c)

Figure 2.4 Spark frequency (a) and oscillation of the voltage (b), equivalent RCL circuit (c)

This can be explained by the equivalent circuit shown in Figure 2.4.c, where $\mathrm{L}$ is the inductance of the cable used. A fit to the damped oscillation corresponding to this circuit is indicated by the second line in Figure 2.4.b.

The oscillation has a period of $0.38 \mu \mathrm{s}$. For a weakly damped oscillator circuit the period is roughly $\mathrm{T}=2 \pi(\mathrm{LC})^{0.5}$. With $\mathrm{C}=2 \mathrm{nF}$ we get $\mathrm{L}=1.9 \mu \mathrm{H}$. This is a realistic value given the length and type of the cable used in our setup. The damping time constant of the fitted curve is $\tau=0.72 \mu \mathrm{s}$. With the formula $\tau=(2 \mathrm{~L} / \mathrm{R})$ for our oscillator circuit we get $\mathrm{R} \approx 5.1 \Omega$, if we regard the spark gap as a resistor in an RCL circuit. This indicates that during the oscillatory period we have a hot discharge of very low resistance, and this is consistent with the assumption of weak damping $\mathrm{R}<2(\mathrm{~L} / \mathrm{C})^{0.5}$. Note that the assumption of a constant $\mathrm{R}$ is a simplification, but the fit agrees well with the measured curve until the measured oscillation stops. The fact the fitted oscillation is not yet damped out at this point indicates that the discharge makes a transition into another mode here. The spikes on the measured curve are a typical indication of this instability. During the oscillatory discharge the energy according to 
equation (2.4) of $\mathrm{E}=3 \mathrm{~mJ}$ is released into the spark during time in order of $2 \mu$ s with the discharge voltage $\mathrm{V}_{\mathrm{d}}=1.8 \mathrm{kV}$.

Note that the timescale of Figure 2.4.b is orders of magnitude smaller than that of Figure 2.4.a. This is why the actual oscillation cannot be observed in Figure 2.4.a. After the strong decrease in voltage associated with the spark discharge, Figure 2.6.a reveals a period of almost constant, slightly decreasing voltage of $\sim 20 \mathrm{~ms}$ after the discharge before the next charging ramp starts again. This strange plateau is associated with the power supply (FUG HCN 14-12500) that was used.

\subsubsection{Experimental Set-up}

In order to investigate the effect of operating conditions on the performance of the spark generator, the experimental setup shown schematically in Figure 2.5 was utilized. It consists of generation, collection, and online particle size distribution measurement units. A stream of 0.8-10 L/min inert gas (less than $1 \mathrm{ppm}$ impurities) is directed towards the gap. Part of this stream permanently flushes the inter-electrode gap. This guarantees that all the ions produced in one spark are removed from the inter-electrode gap before the occurrence of the next spark. It has been pointed out earlier that this leads to a constant discharge voltage (Helsper et al., 1993), and our results confirm this (Figure 2.4.a).

A differential mobility analyser (DMA) combined with an electrometer (AEM) built in our laboratory (Appendix B) measured the particle size distribution on-line. A bipolar neutraliser $\left({ }^{241} \mathrm{Am}\right.$ or $\left.{ }^{210} \mathrm{Po}\right)$ was used ahead of the DMA to bring the particles into a charge equilibrium, which roughly follows a Boltzmann distribution (Hinds, 1999). Data were acquired on a computer using an analog-to-digital converter and a LabView program.

Each size distribution measurement was repeated at least three times to assure repeatability. The uncertainties in the measurements were below 5\%. The particle mass produced per unit time, was measured gravimetrically by collecting the particles on a membrane filter. A Mettler AE200 balance with a readability of $0.1 \mathrm{mg}$ was used. In order to reduce the errors in weight measurements, sampling times were long enough to collect several mg of particles.

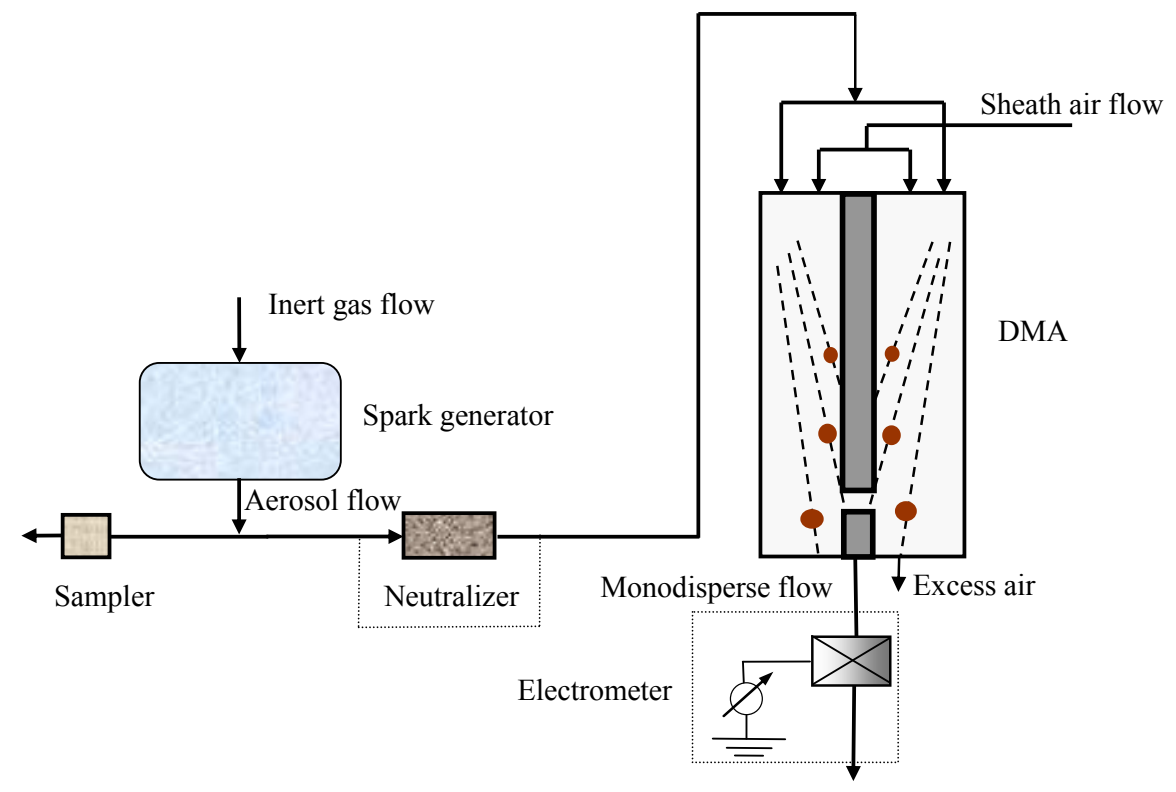

Figure 2.5 Schematic of the experimental set-up 


\subsection{Results and Discussion}

\subsubsection{Effect of Gas Pressure and Type on the Breakdown Voltage}

The breakdown voltage $V_{b}$ has a large influence on the energy delivered to the spark according to equation (2.4). Paschen's law relates $V_{b}$ of a given gas to the product P.d (Va'vra et al., 1998), where $\mathrm{P}$ is pressure and $\mathrm{d}$ is the inter-electrode gap. The spark characteristic is highly sensitive to the gas composition, and the presence of electronegative molecules which capture free electrons has a significant influence on the breakdown process ${ }^{2}$. This is why we did a measurement instead of relying entirely on theory.

Figure (2.6) shows the measured breakdown voltages at different inter-electrode gaps for four gases. As the distance between the electrodes increases, the breakdown voltage also increases. Pressure increase has a similar effect, as can be seen in Figure 2.7. The slopes for air and nitrogen are steeper than those for argon and helium, thus the sparks in air and nitrogen are more strongly affected by the inter-electrode gaps and the gas pressures. The breakdown voltages for air and nitrogen are higher compared to argon and helium, in agreement with theory. The measured values for air are compared with the Paschen curve showing fair agreement at lower P.d. The effects of operating parameters on the particle size distributions will be discussed in the next sections keeping the influence of $\mathrm{P}, \mathrm{d}$ and gas type on the spark energy in mind.

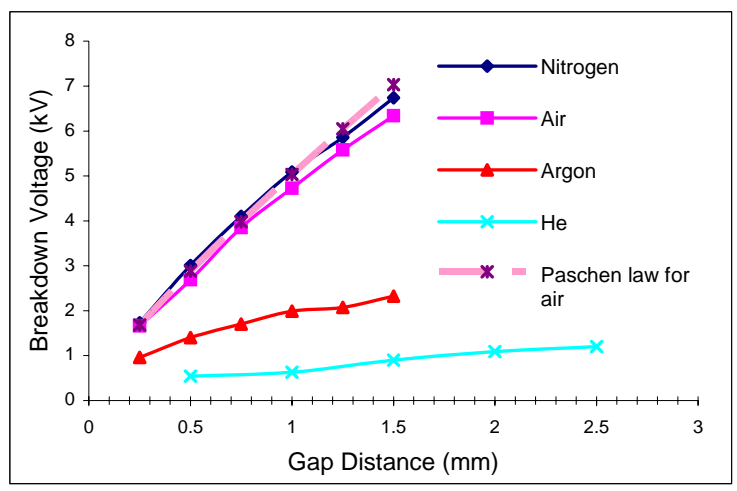

Figure 2.6 Effect of inter-electrode gap on breakdown voltage (1 bar)

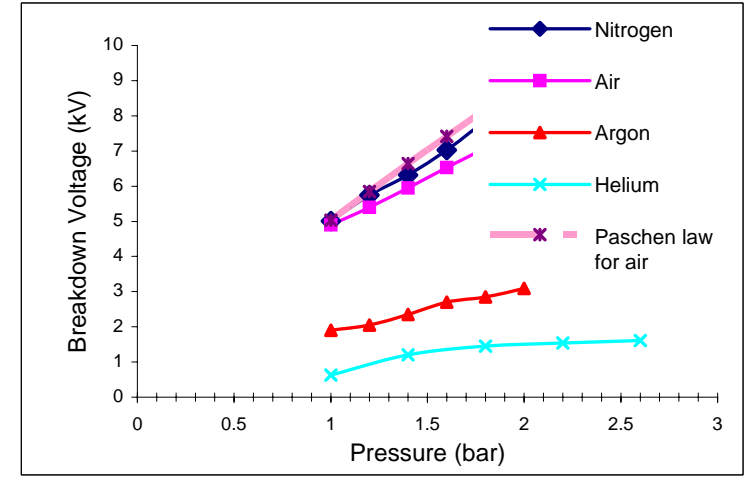

Figure 2.7 Effect of pressure on breakdown voltage (1 mm gap)

\subsubsection{Effect of Gas Pressure on Particle Formation}

The gases applied here, Ar, $\mathrm{He}$ and $\mathrm{N}_{2}$, have different physical, chemical and thermal properties (atomic mass, mean free path, thermal conductivity, electronegativity, etc...) as shown in Table 2.1 .

\footnotetext{
${ }^{2}$ The atoms or molecules in the attaching gases have vacancies in their outermost shells and have affinity for electrons. Attachment represents an effective way of removing electrons which otherwise would have led to current growth and breakdown at low voltages. The electron may directly attach to the gas atom or molecule to form a negative ion $\left(\mathrm{AB}+\mathrm{e} \rightarrow \mathrm{AB}^{-}\right)$, or the gas molecules may split into their constituent atoms and the electronegative atom forms a negative ion $\left(\mathrm{AB}+\mathrm{e} \rightarrow \mathrm{A}+\mathrm{B}^{-}\right)$. ' $\mathrm{A}$ ' is usually sulphur or carbon atom, and ' $\mathrm{B}$ ' is oxygen atom or one of the halogen atoms or molecules (for example $\mathrm{O}_{2}, \mathrm{CO}_{2}, \mathrm{Cl}_{2}, \mathrm{~F}_{2}, \mathrm{C}_{2} \mathrm{~F}_{6}, \mathrm{C}_{3} \mathrm{~F}_{8}, \mathrm{C}_{4} \mathrm{~F}_{10}, \mathrm{CCl}_{2} \mathrm{~F}_{2}$, and $\left.\mathrm{SF}_{6}\right)$, (Naidu, 1995).
} 
Table 2.1 Some properties of the applied gases (Perry, 1984)

\begin{tabular}{|l|l|l|l|l|}
\hline Gas & $\begin{array}{l}\text { Atomic } \\
\text { weight }\end{array}$ & $\begin{array}{l}\text { First ionization } \\
\text { energy }\left(\mathrm{kJ} \mathrm{mol}^{-1}\right)\end{array}$ & $\begin{array}{l}\text { Mean free path } \\
(\mathrm{nm})\end{array}$ & Thermal conductivity $\left(\mathrm{W} \mathrm{m}^{-1} \mathrm{~K}^{-1}\right)$ \\
\hline Nitrogen & 14 & 1402 & 58.8 & $2.59 \times 10^{-2}$ \\
\hline Argon & 40 & 1520 & 62.6 & $1.77 \times 10^{-2}$ \\
\hline Helium & 4 & 2372 & 173.6 & $15.1 \times 10^{-2}$ \\
\hline
\end{tabular}

For example, particle growth is influenced by the cooling effect of collisions of these molecules with the metal vapor. To obtain a better understanding of the gas effect on particle formation, gold particles were produced under different conditions.

TEM micrographs of Au primary particles in nitrogen, argon, and helium at 1 and 2 bars are shown in Figure 2.8. Table 2.2 lists the geometric mean diameters of the primary particles, their geometric standard deviations and the particle number on the TEM images these values are based on. We define the small round particles seen on the micrograph as primary particles. They have been formed by collision of smaller particles and have undergone complete coalescence. Particles formed at a later stage, when the temperature has decreased below the value allowing rapid coalescence, obtain a non-spherical shape.

Further dilution and cooling by the flowing gas takes place outside the spark gap, where room temperature can be assumed. In the diluted state, more agglomeration may occur before deposition of the particles, because the time available is orders of magnitude longer than the time in the hot zone before dilution. Further agglomeration may occur on the sample holder.

Because the particle surfaces are very clean and adsorbate-free, the metal-metal contact has no diffusion barrier and leads to neck formation driven by reduction of surface free energy even at room temperature. This picture is supported by the fact that the surface of particles in the size range of a few nanometers is liquid-like at room temperature (Buffat et al., 1976).
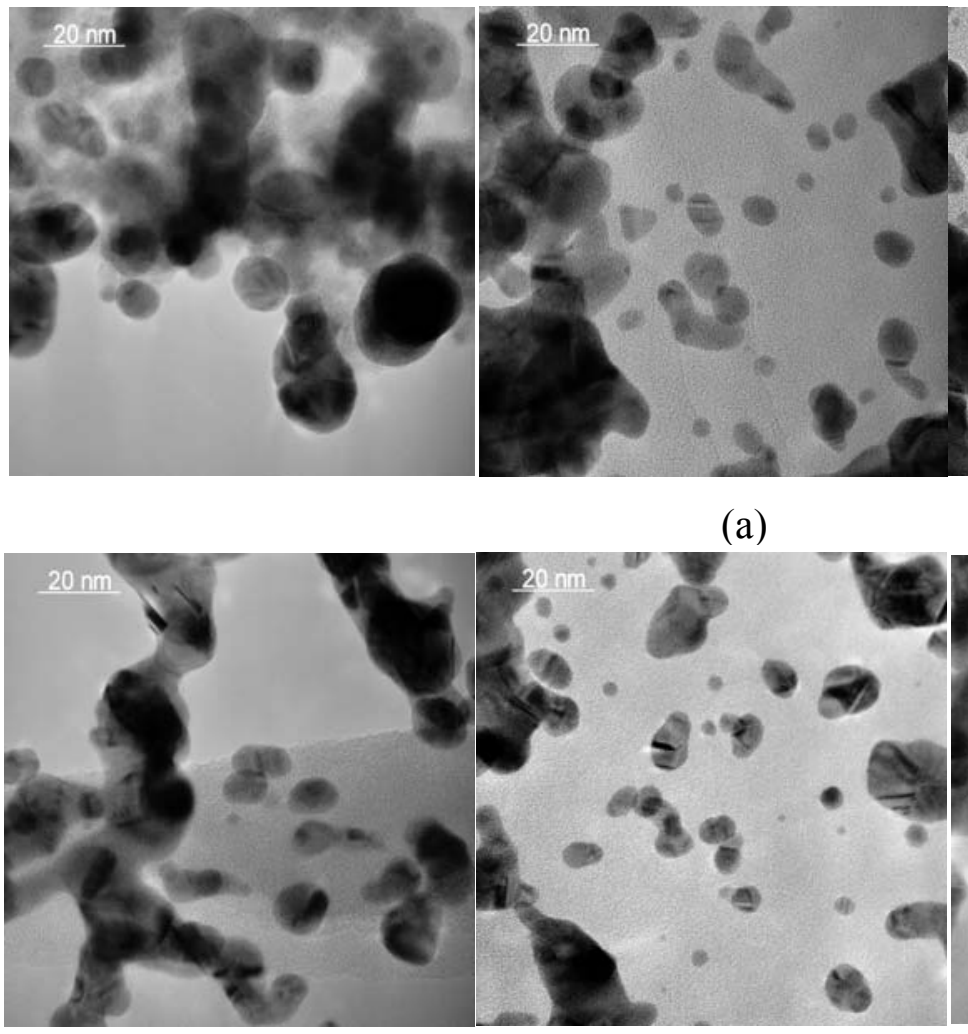

(b)

(a)

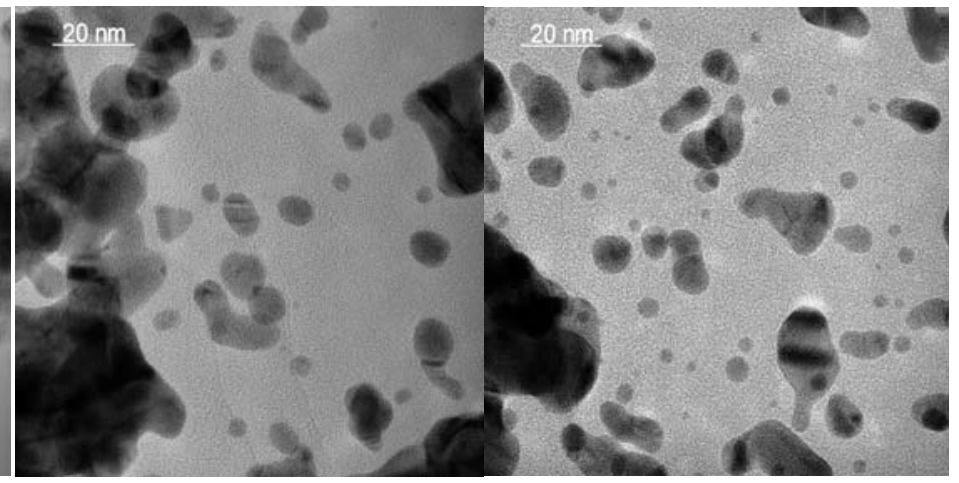

under Nitrogen, Argon, Helium at 1 (top) and 2 bar

Figure 2.8 TEM micrographs of Au particles produced under Nitrogen, Argon,
(down), $(C=20 \mathrm{nF}, d=1 \mathrm{~mm}, f=50 \mathrm{~Hz}, Q=1.2 \mathrm{~L} / \mathrm{min})$ 
Our experiments below show that most of the growth of the large aggregates (sintered agglomerates) seen on the micrographs must be associated with cold sintering.

This is usually not seen in the coagulation of solid particles and is an indication of extreme surface purity. Comparison of the primary particle sizes with the sizes measured by mobility analyser and findings presented below in connection with varied flow velocity support this model.

It was observed that the primary particles produced under $\mathrm{N}_{2}$ were somewhat larger than those produced in the other gases. Surprisingly, doubling the pressure from 1 to 2 bars did not have significant effect on the primary particle sizes.

Table 2.2 Gold particle sizes under various atmospheres

\begin{tabular}{|c|c|c|c|c|c|c|}
\hline & \multicolumn{2}{|c|}{$\mathrm{N}_{2}$} & \multicolumn{2}{c|}{$\mathrm{Ar}$} & \multicolumn{2}{c|}{$\mathrm{He}$} \\
\hline $\mathrm{P}(\mathrm{atm})$ & 1 & 2 & 1 & 2 & 1 & 2 \\
\hline $\mathrm{d}_{\mathrm{g}}(\mathrm{nm})$ & 7.0 & 5.5 & 4.7 & 4.7 & 4.0 & 4.1 \\
\hline$\sigma_{\mathrm{g}}$ & 1.34 & 1.35 & 1.42 & 1.37 & 1.45 & 1.4 \\
\hline Count & 35 & 37 & 40 & 44 & 69 & 36 \\
\hline
\end{tabular}

Due to the low energy per spark in He, the particle concentrations were relatively small and could hardly be measured precisely. Typical size distributions of particles produced under Ar and $\mathrm{N}_{2}$, measured by the DMA below (Figure 2.9), showed a larger modal diameter for particles produced under $\mathrm{N}_{2}$ as compared to Ar which is in qualitative agreement with Table 2.1. The large aggregates seen in Figure 2.8 exceed the sizes typically seen in the DMA spectra by far and therefore they have likely been formed on the substrate (Gleiter et al., 2001).

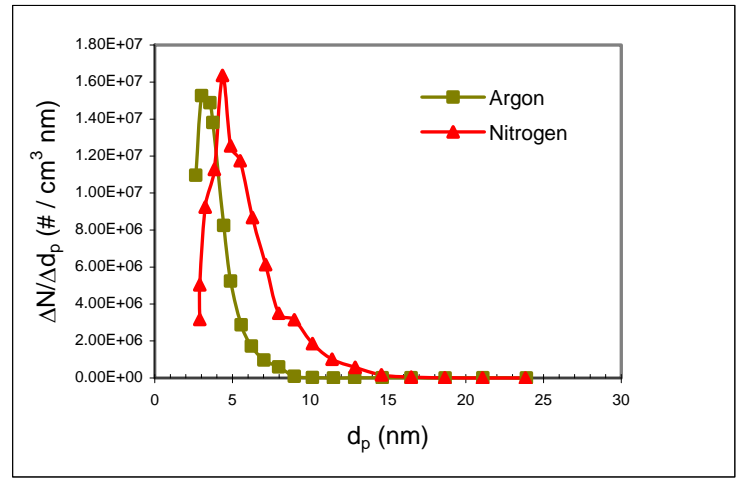

Figure 2.9 Size distributions of particles produced under $\mathrm{N}_{2}$ and $\mathrm{Ar}$ $(C=5 \mathrm{nF}, d=1 \mathrm{~mm}, f=10 \mathrm{~Hz}, Q=10 \mathrm{~L} / \mathrm{min})$

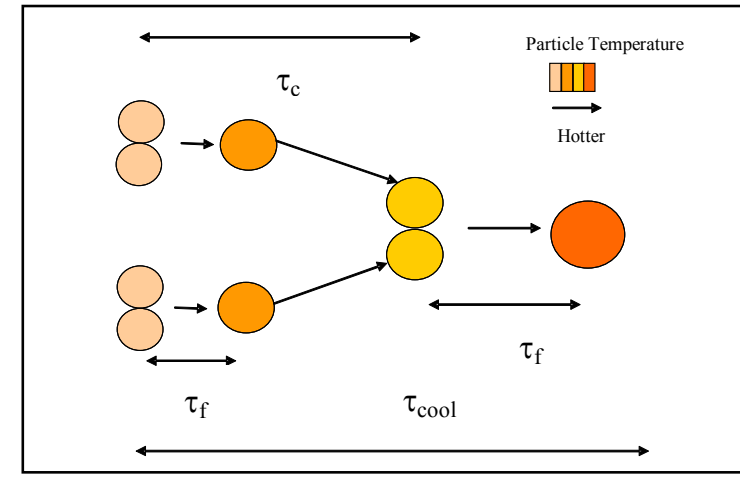

Figure 2.10 Collision, coalescence, and cooling at early stages of particle growth (reproduced from Lehtinen, 2002)

Since these are sintered agglomerates (aggregates), this confirms the assumption of sintering at room temperature.

The following considerations will give a qualitative explanation for the present experimental findings. At sufficiently high temperatures in the initial phase of particle formation, coalescence is fast enough to form spherical particles. At lower temperatures, where coalescence is slow, particle contact evidently forms atomic bonds under the present clean surface conditions, even at room temperature, and necks between primary particles occur, but the particles formed are non-spherical. The further process of coalescence is so slow, so that the fractal-like aggregate shape is frozen (Swihart, 2003).

In the case that is more common than the present one, loose agglomerates are formed, where the primary particles are held together by van der Waals forces. This occurs for most materials, especially where surface oxidation or other adsorbates avoid a direct metal-metal 
contact. For further understanding of our results, particularly the weak dependence of the primary particle size on pressure, the particle formation process can roughly be described in the following way: The discharge locally heats up the gas to a high temperature in the spark channel. The electrodes are locally heated to above the boiling point on areas of several square micrometers.

As in laser ablation, this produces small vapor plumes close to the electrodes that are initially cooled by radiation and adiabatic expansion and subsequently by diffusional mixing with the surrounding cold gas. Particle formation occurs well below the boiling point, where mixing simultaneously reduces the temperature. Because of the extremely high initial temperature, it is certainly correct to state that the plume is initially pure vapor and that particle formation takes place under conditions of "high loading".

It is easily shown that this would even be the case if cooling would exclusively be due to mixing. Lehtinen and Zachariah (2002), have shown that under high enough volume loading conditions the heat release of colliding particles due to the reduction in the free energy plays a decisive role, because the time for cooling due to collisions with gas molecules is longer than the time between two collisions. This leads to particle heating, which in turn guarantees complete coalescence between collisions.

Figure 2.10 illustrates the process assumed by Lehtinen et al. (2002), according to which the cooling time $\tau_{\text {cool }}$ is larger than the collision time. Because of the particle heating effect, the coalescence (fusion) time $\tau_{\mathrm{f}}$, remains smaller than the collision time $\tau_{\mathrm{c}}$. This guarantees coalescence until $\tau_{\text {cool }}$ drops below $\tau_{\mathrm{c}}$, because mixing with the cold gas reduces the cooling time and increases the collision time. Another effect continuously increasing the collision time is coagulation itself, of course. When $\tau_{\mathrm{cool}}<\tau_{\mathrm{c}}$, particle temperature falls and coalescence is stopped. This marks the end of primary particle growth, and subsequent collisions lead to non-spherical particles. According to this model, the final primary particle size thus decreases with higher gas pressure (enhanced cooling) and grows with volume loading. This delivers a qualitative explanation for the weak dependence of the primary particle size on pressure seen in Table 2.1: pressure increase leads to a higher breakdown voltage, increasing the spark energy and thus the metal loading. According to Lehtinen et al. (2002), this favours a larger primary particle size, but the increased cooling associated with the higher pressure evidently compensates this effect. Looking at the dependence of the primary particle size on the type of gas used, the largest particles are produced in nitrogen, because this gas has the highest breakdown voltage leading to higher loading and thus larger particles in agreement with the model. Increase of metal loading via the spark energy by using a higher capacitance leads to the same qualitative results, as shown in Figure 2.11 obtained from electron microscopy.

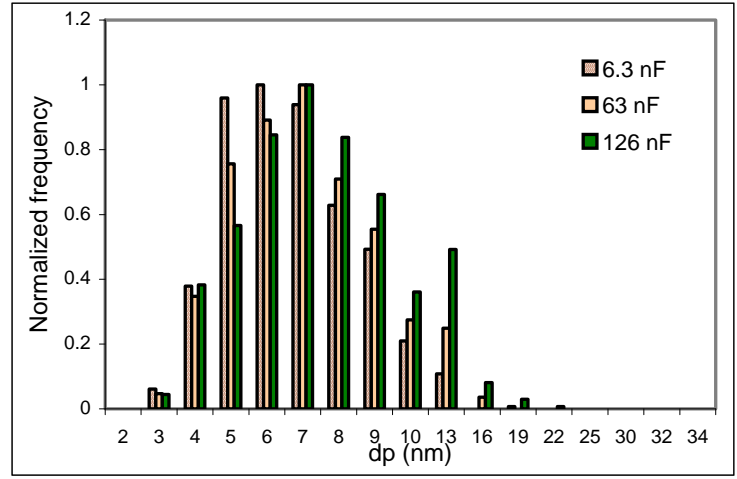

Figure 2.11 Variation of gold primary particle sizes with capacitance under $\mathrm{N}_{2}$

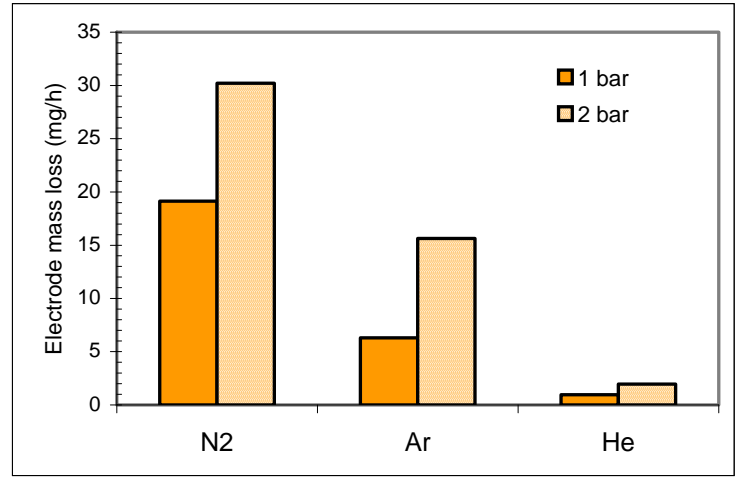

Figure 2.12 Gold particle production rates under $\mathrm{N}_{2}, \mathrm{Ar}, \mathrm{He}$ $(C=20 \mathrm{nF}, d=1 \mathrm{~mm}, f=100 \mathrm{~Hz}, Q=0.8 \mathrm{~L} / \mathrm{min})$ 
The particle mass production rates at the same frequency, gap distance and capacitance, under different atmospheres at 1 and 2 bars are shown in Figure 2.12. In each measurement the electrodes were carefully wiped off to remove the loose particles and weighed to determine the mass loss. It can be seen that the production rates increase with pressure. This is expected, because the breakdown voltage and consequently the spark energy, increases with pressure.

\subsubsection{Effect of Gas Flow Rate on Particle Formation}

The gas flow rate is expected to influence cooling as well as dilution. Increased gas flow should therefore enhance both the cooling and dilution rate, leading to smaller primary particles. Interestingly, the particle size measured by DMA remains constant when the flow rate is increased beyond $5 \mathrm{~L} / \mathrm{min}$, as Figure 2.14 shows. We explain this by assuming two different particle growth domains. A high concentration domain between the electrodes, where flow can be assumed laminar, and a low-concentration domain after turbulent mixing with the flow, which takes place after leaving the space between the electrodes. The fact that there is hardly any influence of the flow velocity above $5 \mathrm{~L} / \mathrm{min}$ is explainable, if particle formation essentially takes place in the high concentration laminar flow region. Here dilution is dominated by diffusion and thus flow independent, and vertical displacement of the particle formation zone has no influence on the process. This means that particle formation is essentially completed in the laminar flow zone, and the particle diameter that develops here is close to the primary particle diameter. After turbulent dilution, the time available for further coagulation is orders of magnitude longer, and the rise of particle size when the flow rates becomes smaller than $5 \mathrm{~L} / \mathrm{min}$ is attributed to coagulation in the diluted region. From Figure 2.13 we infer that the geometric standard deviation of the size distribution is 1.28 for 5 $\mathrm{L} / \mathrm{min}, 1.34$ for $3 \mathrm{~L} / \mathrm{min}$, and 1.48 for $1.5 \mathrm{~L} / \mathrm{min}$. The latter value is in agreement with the self -preserving distribution for spherical particles in the free molecular range, which is 1.46 and higher for deviations from spherical shape (Vemury et al., 1995). Interestingly, the size distribution is narrower for larger flow, i.e. shorter coagulation in the diluted zone. The results thus indicate that the primary particle size distribution is significantly narrower than the self-preserving value. The subsequent size distribution measurements were carried out at relatively high flow rates of argon (around 5 to $10 \mathrm{~L} / \mathrm{min}$ ), in order to reflect the primary particle growth process rather than cold agglomeration of the diluted aerosol.

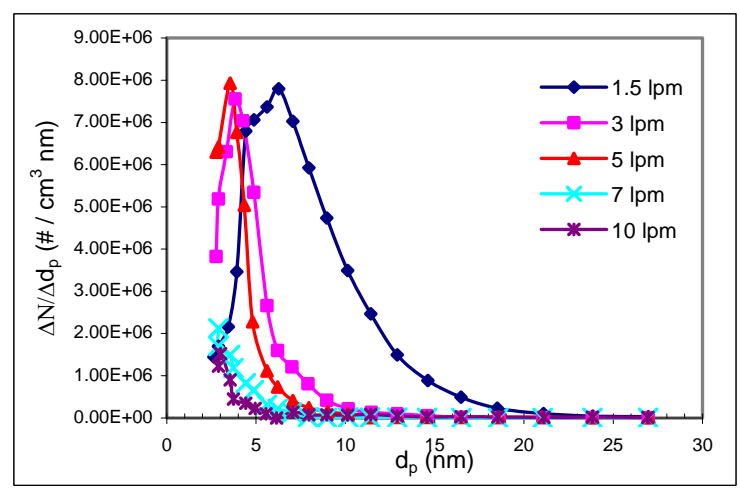

Figure 2.13 Particle size distributions at different gas flow rates $(C=5 \mathrm{nF}, d=0.5 \mathrm{~mm}, f=10 \mathrm{~Hz}, \mathrm{Ar})$

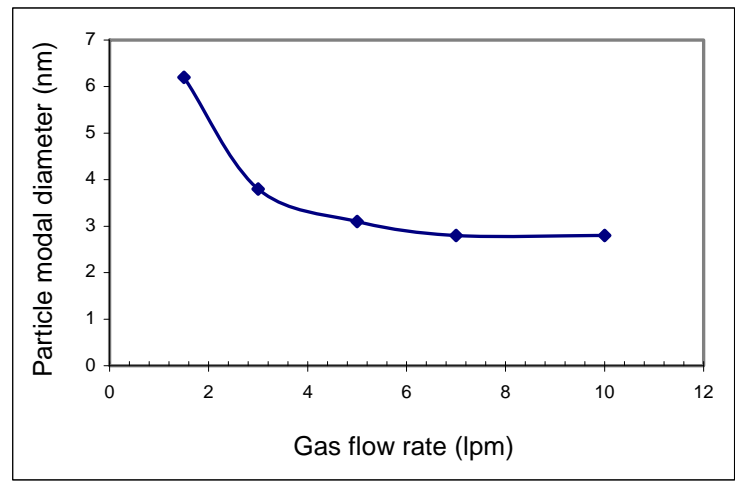

Figure 2.14 Particle modal diameters at various gas flow rates $(C=5 \mathrm{nF}, d=0.5 \mathrm{~mm}, f=10 \mathrm{~Hz}, \mathrm{Ar})$ 


\subsubsection{Effect of Inter-Electrode Gap on Particle Formation}

The relation between the gap distance and spark energy and the data of Figure 2.6 for argon is shown in Figure 2.15. We replaced the breakdown voltage by the discharge voltage, assuming that the overvoltage is small. The spark energy increases with the gap distance, but we expect that energy transfer to the particle production process will be less efficient. To examine the effect of gap distance on the particle mass production rate, the electrode mass losses at different gap distances were measured. The electrode mass loss increased with the gap distance, as shown in Figure 2.16, but the particle mass produced per unit spark energy $(\mathrm{kWh})$ decreased according to Figure 2.17. As the gap grows, more energy is necessary to produce the same mass. This is qualitatively understandable, because the spark energy is distributed over a larger volume if the distance is large, and less efficiently used for evaporation of the electrodes.

The size distributions of particles produced with different gap distances can be seen in Figure 2.18. As the gap distance grows, the mean diameters of the size distribution curves increase, as the model (Lehtinen et al., 2002) predicts for higher vapour loading.

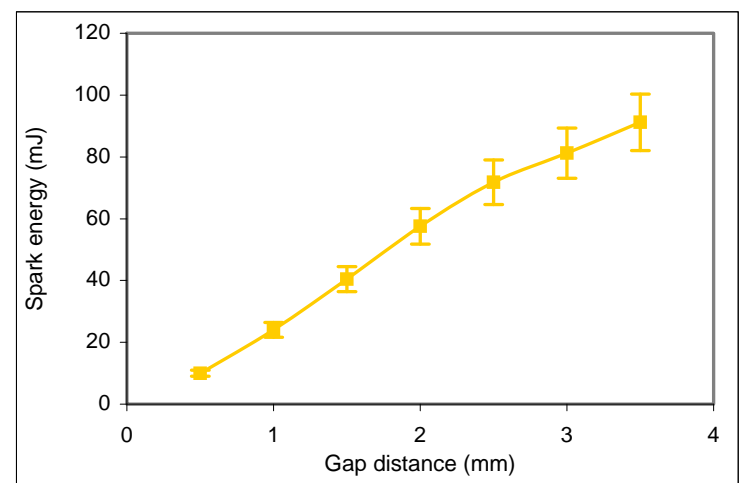

Figure 2.15 Spark energies at different gap distances under Argon

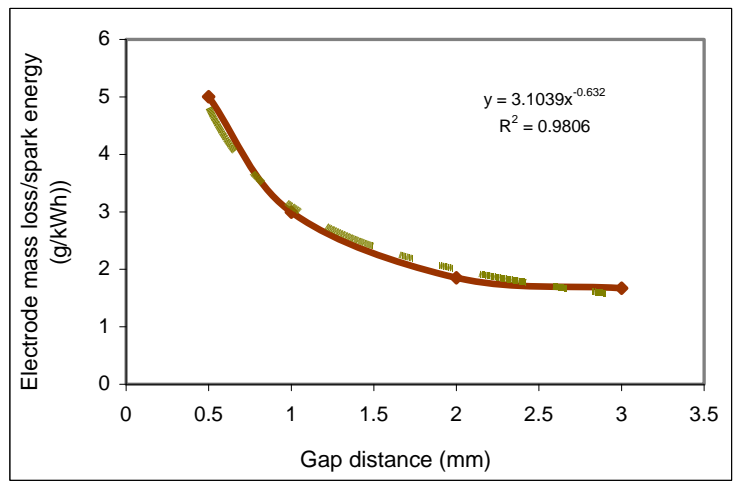

Figure 2.17 Electrode mass losses per unit spark energy at different gap distances

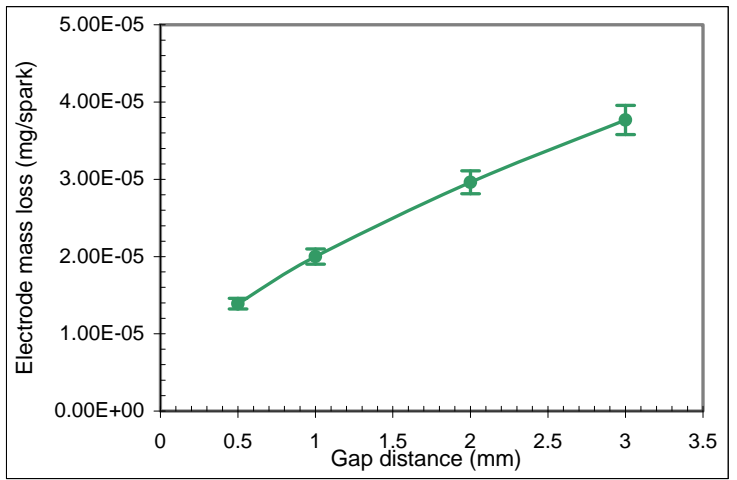

Figure 2.16 Electrode mass losses per spark at different gap distances $(\mathrm{C}=20 \mathrm{nF}, \mathrm{f}=150 \mathrm{~Hz}, Q=0.8 \mathrm{~L} / \mathrm{min}, \mathrm{Ar})$

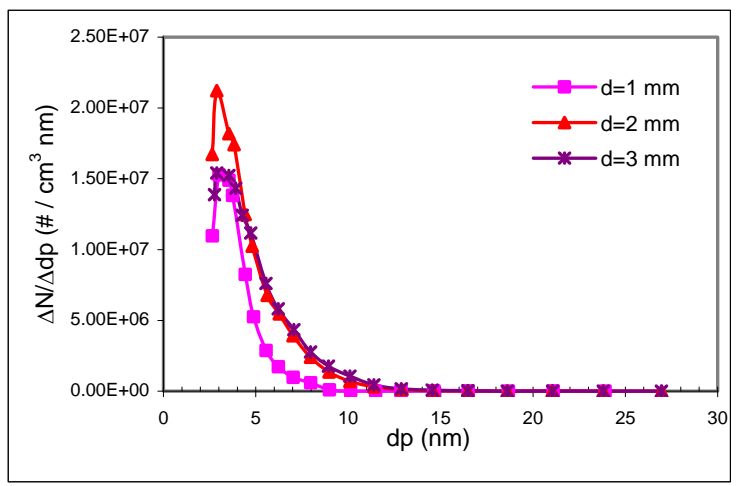

Figure 2.18 Particle size distributions at different gap distances $(C=5 \mathrm{nF}, f=10 \mathrm{~Hz}, Q=10 \mathrm{~L} / \mathrm{min}, \mathrm{Ar}$ ) 


\subsubsection{Effect of Capacitance on Particle Formation}

Since spark energy is directly proportional to the capacitance (equation 2.4), increasing the capacitance results in stronger spark and therefore higher metal vapour loading in particle growth region. Size distributions of particles produced at different capacitances are presented in Figure 2.19, indicating that the modal diameters and particle concentrations increase with the capacitance. TEM analysis results for gold particles produced under $\mathrm{N}_{2}$ in Figure 2.11, qualitatively supports these DMA results.

Variation of mass production rate with the capacitance can be seen in Figure 2.20. The result is consistent with proportionality. This means that with a fixed gap, the fraction of energy that is used for evaporation is constant. The energy per spark can be calculated using equation (2.1), assuming that the residual voltage $\mathrm{V}_{0}$ is negligible and that the discharge voltage equals the break-down voltage.

Using the constant ratio of the electrode mass loss and the capacitance from Figure 2.20 and considering the enthalpy of evaporation for gold $(330 \mathrm{~kJ} / \mathrm{mol})$ we conclude that less than $0.1 \%$ of the spark energy is consumed for particle production. This estimation assumes that the entire electrode mass loss is due to material evaporation and ignores the material transfer between the electrodes (see below).

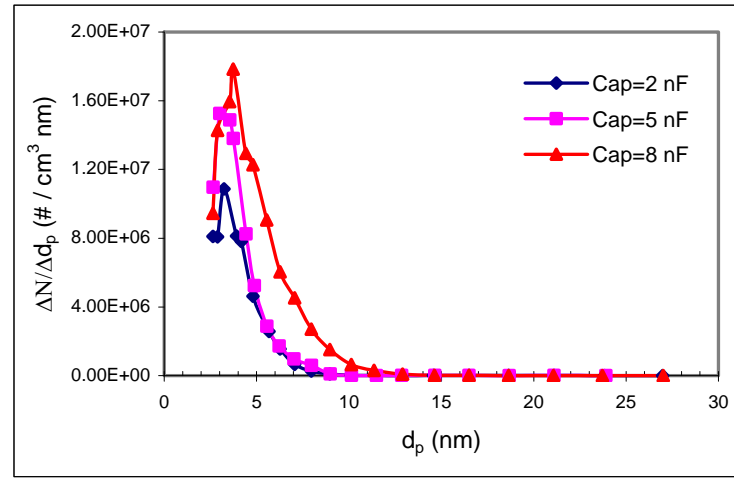

Figure 2.19 Effect of capacitance on the particle size distribution

(d=1 mm, $f=10 \mathrm{~Hz}, Q=10 \mathrm{~L} / \mathrm{min}, \mathrm{Ar}$ )

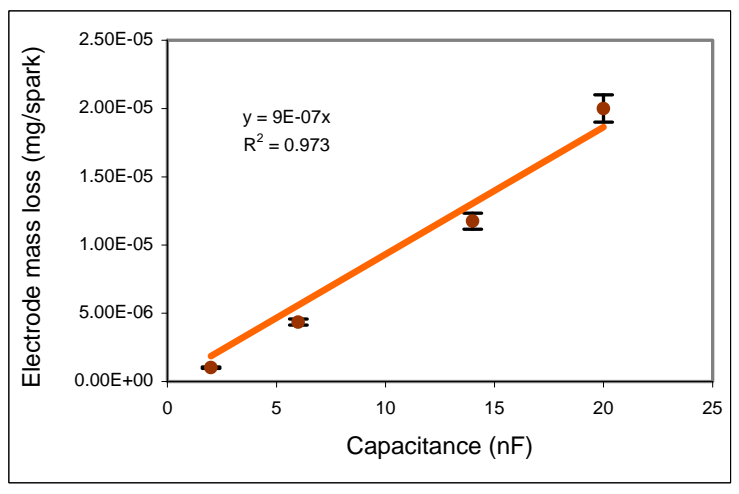

Figure 2.20 Effect of capacitance on mass production rate (d=1 mm, $f=150 \mathrm{~Hz}, Q=0.8 \mathrm{~L} / \mathrm{min}, \mathrm{Ar})$

\subsubsection{Effect of Electrode Material on Particle Formation}

While a major advantage of the spark discharge method is its versatility concerning materials, it is clear that the material must have significant influence on the particle formation process. The spark strikes the electrode and electrical energy is transformed into thermal energy, causing local evaporation of the electrodes ${ }^{3}$ as in laser ablation. Thus particle formation is certainly influenced by thermal properties of the electrode material like the boiling point and the evaporation enthalpy. Besides, properties that influence the spark formation and characteristics for example the work function for electron emission have an influence (Naidu, 1995) as well as optical properties, determining absorption of the spark's radiation. Size

\footnotetext{
${ }^{3}$ In micro-EDM, $\mathrm{KT}_{\mathrm{m}}$ ( $\mathrm{K}$ is the thermal conductivity and $\mathrm{T}_{\mathrm{m}}$ the melting temperature), has been used as a measure of wear resistance of the electrode material (Tsai and Masuzawa, 2004).
} 
distributions of particles produced from various electrode materials at the same operating conditions were measured with the DMA. As seen in Figure 2.21, the size distributions strongly depend on material. Electrode material loss measurements showed that tungsten, possessing the highest evaporation enthalpy per mole was the most spark resistant material, while antimony and magnesium having the lowest evaporation enthalpy per mol showed the highest spark erosions (Figure 2.22). Assuming that the effective area for energy transfer is the area of the hot spots $\mathrm{A}\left(\mathrm{m}^{2}\right)$ on the cathode and anode (Soldera et al., 2005), the energy balance around the hot spots can be estimated by the following equation, which relates the eroded mass due to the evaporation to the physical properties of the electrode material (Llewellyn Jones, 1950):

$$
m=\frac{\frac{1}{2} c_{e} V^{2}-b T_{b}^{4}-g k\left(T_{b}-T\right)}{c_{p s}\left(T_{m}-T\right)+\Delta H_{m}+c_{p l}\left(T_{b}-T_{m}\right)+\Delta H_{v}}
$$

The first term in the numerator represents the effective spark energy, with $c_{e}(F)$ representing the effective gap capacitance and $\mathrm{V}$ the breakdown voltage $(\mathrm{V})$. The second term denotes the heat loss from the hot spots by radiation in which $T_{b}(k)$ is the boiling point and $b=A \sigma t\left(\mathrm{Jk}^{-4}\right)$ with $\sigma$ depending on the blackness of the metal (for black bodies $\sigma$ is Stefan-Boltzmann constant $5.67 \times 10^{-8} \mathrm{Js}^{-1} \mathrm{~m}^{-2} \mathrm{k}^{-4}$ ). The time for energy transfer is $\mathrm{t}$. The third term indicates the heat transfer from the hot spots by conduction, in which $\mathrm{k}$ is the thermal conductivity $\left(\mathrm{Wm}^{-}\right.$ $\left.{ }^{1} \mathrm{k}^{-1}\right)$ and $\mathrm{g}=2(\pi \mathrm{A})^{0.5} \mathrm{t}(\mathrm{ms})$ if $\left(\mathrm{r}^{2} / \mathrm{Kt}\right)^{0.5}<<1$, in which $\mathrm{K}$ is the thermal diffusivity $\left(\mathrm{m}^{2} \mathrm{~s}^{-1}\right)$ and $\mathrm{r}$ the radius $(\mathrm{m})$ corresponding to $\mathrm{A}$. $\mathrm{T}$ is the steady state temperature $(\mathrm{K})$ far from the hot spot (e. i. ambient temperature). The first and second terms in the denominator represent the energy required to heat the electrode material to the melting point $T_{m}(K)$, with $C_{p s}\left(J^{-1} k^{-1}\right)$ the average heat capacity of the solid, and $\Delta \mathrm{H}_{\mathrm{m}}\left(\mathrm{Jg}^{-1}\right)$ the enthalpy of melting. The third term in denominator is the energy needed to heat the liquid to the boiling point, with $\mathrm{C}_{\mathrm{pl}}\left(\mathrm{Jg}^{-1} \mathrm{k}^{-1}\right)$, the average heat capacity of the liquid, and $\Delta \mathrm{H}_{\mathrm{v}}\left(\mathrm{Jg}^{-1}\right)$ the enthalpy of evaporation. The evaporated mass is denoted by $\mathrm{m}(\mathrm{g})$. The model excludes any chemical reaction and ignores material transfer between the electrodes. The material constants in the equation are known. The constants $c_{e}, b$ and $g$ are assumed independent of the electrode material. Since A and $t$ are not accurately known, we estimate these parameters empirically by fitting equation (2.7) to our measured mass loss data of Figure 2.22. The discrepancies between theory and experiment are large, but it should be considered that the model used oversimplifies the process (Meek et al., 1953). Making the further simplifying assumption that the electrodes are black bodies gives us the values $\mathrm{r} \sim 1.5 \mu \mathrm{m}$ and $\mathrm{t} \sim 1.2 \mu \mathrm{s}$. Both values are in the expected range.

The analysis implies that thermal energy transfer through heat conduction plays a more important role than radiation. Materials with higher thermal conductivity show less evaporation, because they are cooled more effectively (Szenete et al., 1994). Equation (2.7) correctly reflects our results in an approximate sense with respect to heat conduction, boiling temperature and evaporation enthalpy. It can therefore be used to predict the mass production rate for any material at the identical gap distance and spark type. 


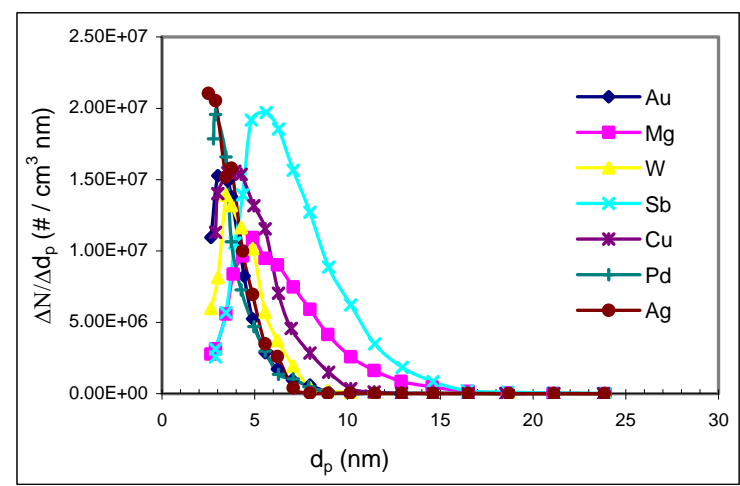

Figure 2.21 Size distributions of particles produced from various materials $(C=5 \mathrm{nF}, d=1 \mathrm{~mm}, f=10 \mathrm{~Hz}, Q=10 \mathrm{~L} / \mathrm{min}, \mathrm{Ar})$

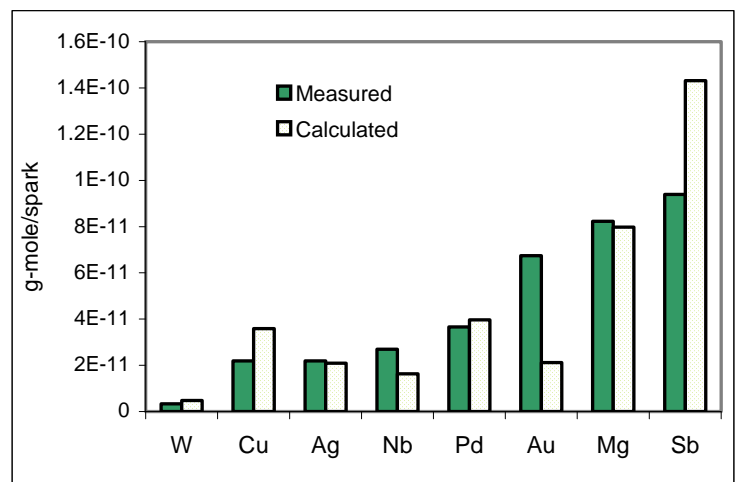

Figurw 2.22 Electrode material losses for various electrodes

$(C=20 \mathrm{nF}, d=1 \mathrm{~mm}, f=100 \mathrm{~Hz}, Q=0.8 \mathrm{~L} / \mathrm{min}, \mathrm{Ar})$

\subsubsection{Effect of Spark Repetition Frequency on Particle Formation}

The particle mass production is adjustable over a wide range by means of the spark frequency, which is given by the electric current via equation (2.6). It can be seen from Figure 2.24 that the particle production rate linearly increases with the spark frequency and the production rate can conveniently be controlled through the frequency. This is expected, if the spark energy is independent of frequency and each spark produces particles independently. Figure 2.23 shows size distributions obtained at different repetition frequencies. The distributions widen with increasing frequency, which indicates coagulation. The particle concentration first rises with frequency but decreases at high frequency. This is explainable by the fact that at higher frequencies more coagulation takes place at high concentration and before dilution is complete. If coagulation of the primaty particles is to be avoided at increased frequency, this could be done by increasing the flow, in order to reach the plateau as in Figure 2.14.

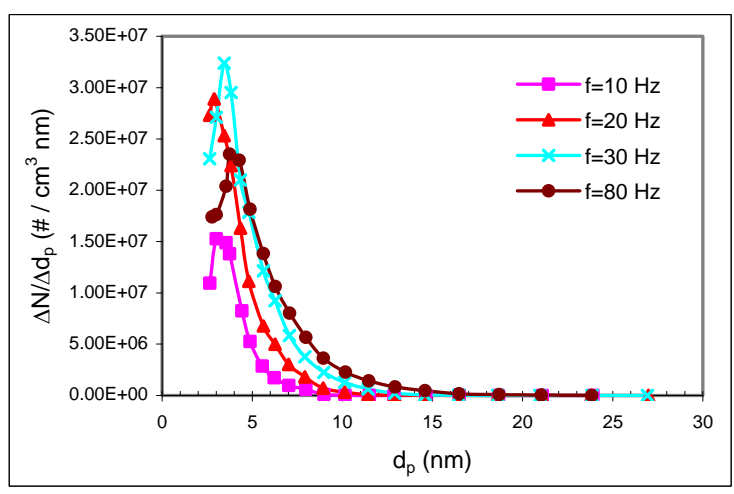

Figure 2.23 Effect of frequency on the particle size distribution (C=5 $\mathrm{nF}, d=1 \mathrm{~mm}, Q=10 \mathrm{~L} / \mathrm{min}, \mathrm{Ar}$ )

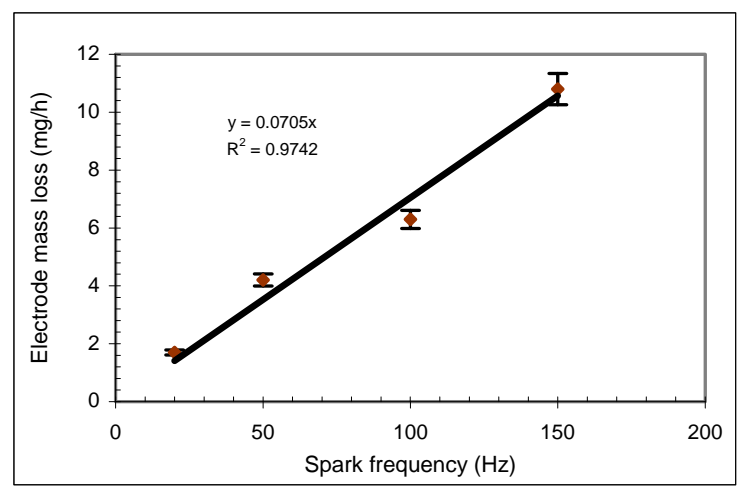

Figure 2.24 Effect of frequency on gold particle production rate $(C=20 \mathrm{nF}, d=1 \mathrm{~mm}, Q=0.8 \mathrm{~L} / \mathrm{min}, \mathrm{Ar})$ 


\subsubsection{Charge State of Particles}

Since plasma is an environment containing electrons and ions, it is likely that the particles produced by spark discharge obtain charge during the synthesis process. Indeed our measurements showed that the spark discharge generator produced charged particles with both polarities. In most cases there was a higher concentration of negative particles than positive particles. Figures 2.25-28 show the size distributions of gold and silver negatively and positively charged particles exiting directly from the spark generator as well as the size distributions of all particles. The size distributions of the charged particles were determined bypassing the neutralizer and calculated under the assumption that each particle carries a single elementary charge. To obtain the size distribution of all particles, the neutralizer was used. It establishes a well-known equilibrium charge distribution on the particles according to the extended theory by Fuchs (Fuchs, 1963; Wiedensohler et al., 1991) and this enables calculation of the size distribution of all particles, regardless of their initial charge. In both cases the concentration of negative particles was larger than the positive particles. This may be attributed to electrostatic precipitation of positive particles to the walls and housing, since in our setup the positive high voltage was connected to one of the electrodes while the other electrode and the housing were grounded. Thus there is an electrostatic force on the positive particles towards the housing, while the negative particles are repelled and survive with a higher probability. Precipitation of particles to the electrodes also occurs, as can be seen from the deposits. Figure 2.26 shows that, about $20 \%$ of the particles obtain a negative polarity, and for the smallest particles, this percentage even looks significantly higher. This is of great significance in view of electrostatic size classification. This technique suffers from the low charging efficiency of available chargers, and a production technique that delivers a large fraction of charged particles of one polarity is highly desirable. The charging technique most commonly used is bipolar charging, which is precisely what our neutralizer does. In Figures $2.25,2.27$ the size distributions of positive and negative particles from the spark generator are compared with those when using the bipolar charger. The negative particle yield is 3 times higher, when the original charge from the spark generator is used, and this ratio is apparently still much higher for the smallest particles of the distribution.

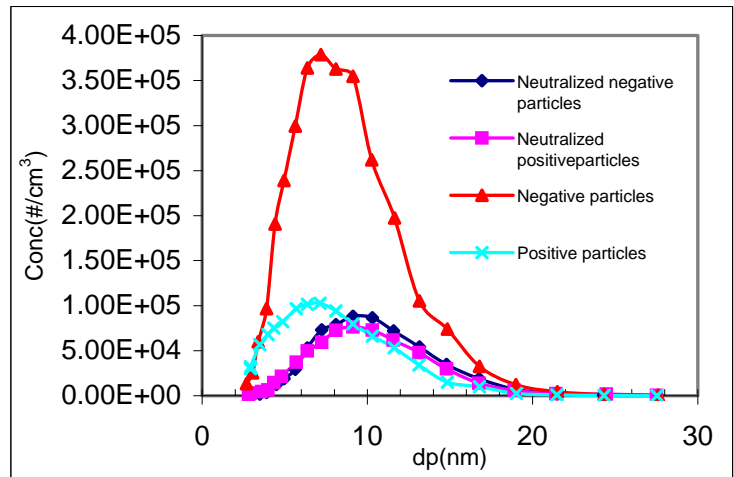

Figure 2.25 Size distributions of Au neutralized and non-neutralized particles

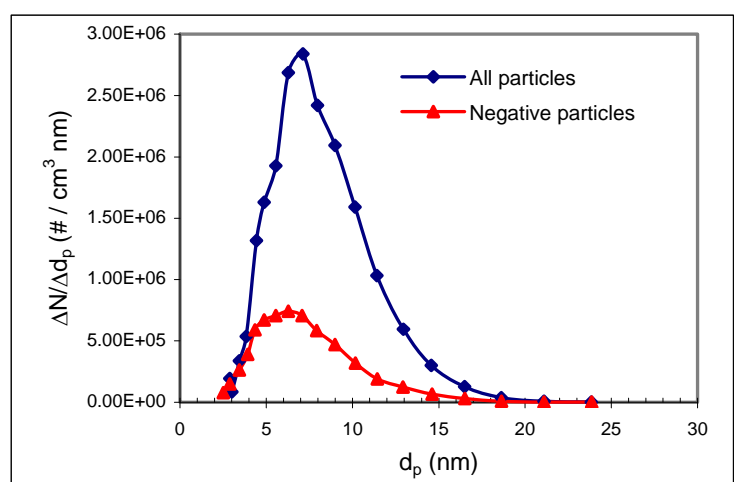

Figure 2.26 Size distributions of total and negative Au particles $(C=5 \mathrm{nF}, d=1 \mathrm{~mm}, f=10 \mathrm{~Hz}, Q=5 \mathrm{~L} / \mathrm{min}, \mathrm{Ar})$ 


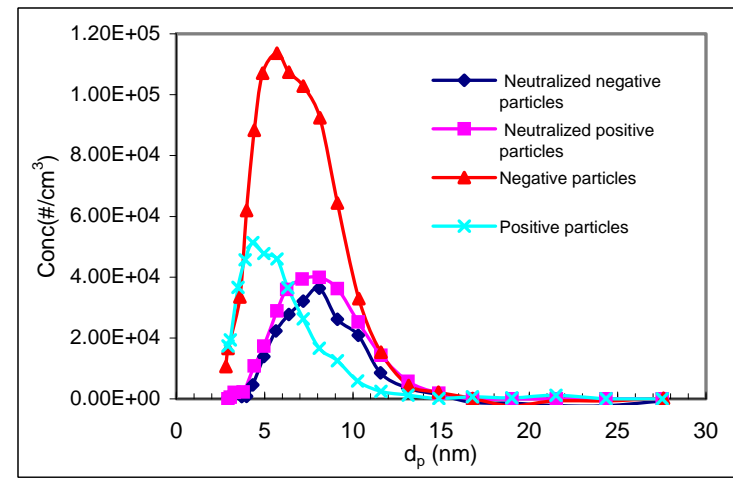

Figure 2.27 Size distributions of Ag neutralized and non-neutralized particles

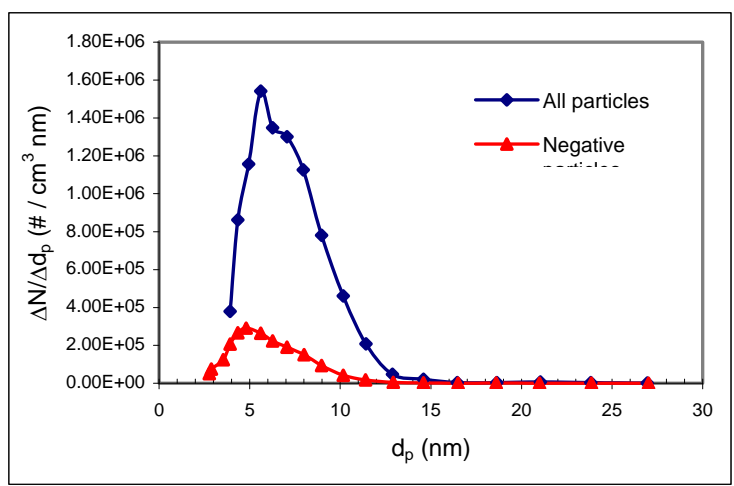

Figure 2.28 Size distributions of total and negative Ag particles $(C=5 \mathrm{nF}, d=1 \mathrm{~mm}, f=10 \mathrm{~Hz}, Q=5 \mathrm{~L} / \mathrm{min}, \mathrm{Ar})$

\subsubsection{Particle Losses in the Present Set-up}

From the size distribution data, the mass of the gold particles was estimated. The ratio of this estimation and the gold electrode mass loss was around 5-10\%. For gold, silver and palladium electrodes the particles were collected on membrane filters mounted in an airtight stainless steel holder at the exit of the generator. The ratios of the collected masses and the electrode mass losses were determined to be $22-38 \%$. Thus we lose at least $62 \%$ in the generator and in the ducts. These losses are mainly due to diffusion and would be avoidable by minimizing the transport times (faster flow). For the charged particles we believe that electrostatic precipitation in the generator is at least as important.

\subsubsection{Material Transfer between Electrodes}

It has been reported that the vaporized electrode material travels into the spark gap in the form of a jet, which is observable via the radiation of the excited metal atoms (Cundall et al., 1955). Material transfer between electrodes can occur when the vapor jet from one electrode reaches the other one (Figure 2.29). This causes a reduction in the net mass loss (Cundall et al., 1955). This fact should particularly be considered in applications where two compositionally different electrodes are used, because the jet has a mixing effect that can be used for production of bimetallic particles (see chapter 4). Efficient mixing of the vapors is possible, if the vapor jets coincide in the inter-electrode gap.

Figure 2.30.a demonstrates material transfer from the cathode $(\mathrm{Cu})$ to the anode electrode (W). In the course of the spark discharge, electric current is converted into heat and the electrode surface is intensively heated in the area of the discharge channel. This results in the formation of a liquid pool, from where the molten metal either evaporates or is explosively ejected into the surrounding fluid, forming craters on the electrode surface. The area of these craters is likely the area of energy transfer (Soldera et al., 2005; Llewellyn Jones, 1950) referred to in the energy balance model above. Figure 2.30.b shows the intense erosion of the cathode $(\mathrm{Cu})$.

Figure 2.31 shows the surface of the gold anode electrode interacted with the plasma. It can be seen that platinum from the cathode has been transferred to the gold electrode manifested in the picture as silverish dots. The inset shows the hills created on the gold electrode surface. 

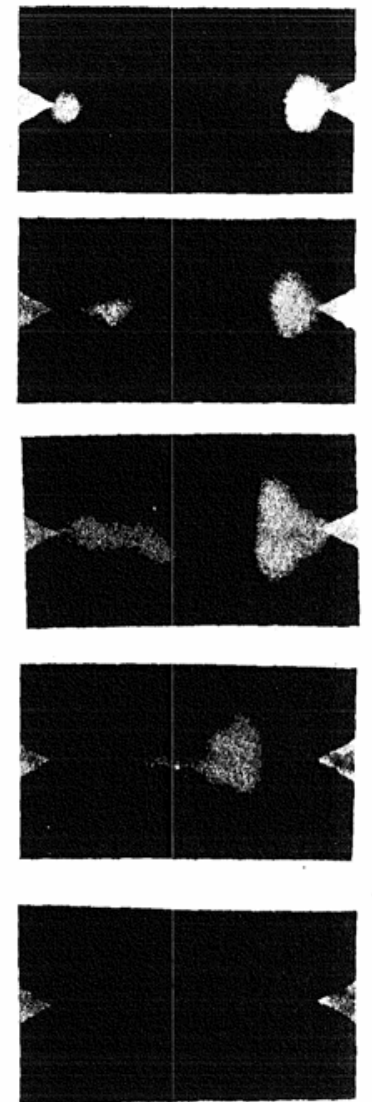

(a)

ANODE

CATHODE
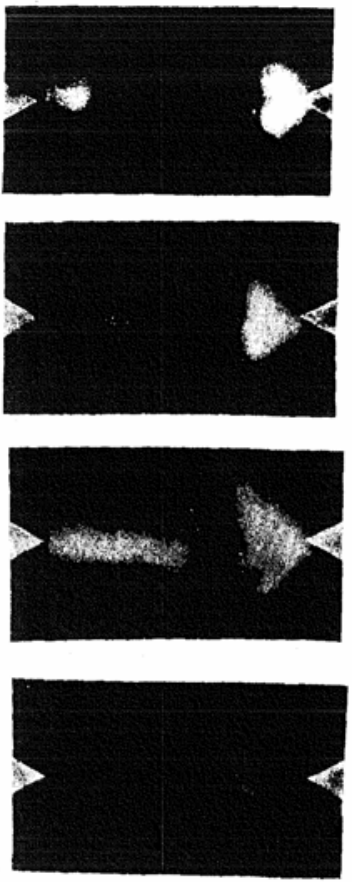

(b)

Figure 2.29 Image converter photographs of 600-amp, 3- $\mu$ sec spark between Zn electrodes, 0.5 usec exposure (by courtesy of Cundall, 1955)

Redeposition of the nanoparticles on the electrode surface can also be seen as black regions in the inset.

To study the relative spark abrasion of the electrodes, the mass loss was individually measured for each electrode under approximately constant conditions (Figure 2.32). Noble metals were selected to avoid oxidation. In all the cases it was observed that mass loss for the negative electrode was larger compared to the positive electrode.

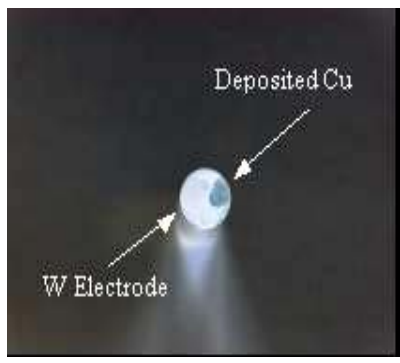

(a)

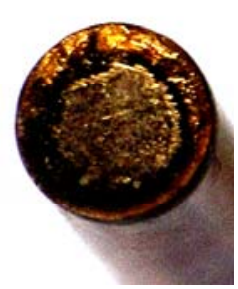

(b)

Figure 2.30 Material transfer from $\mathrm{Cu}$ electrode to $W$ electrode in $[W(+) C u(-)]$ system (a), $\mathrm{Cu}$ electrode erosion (b)

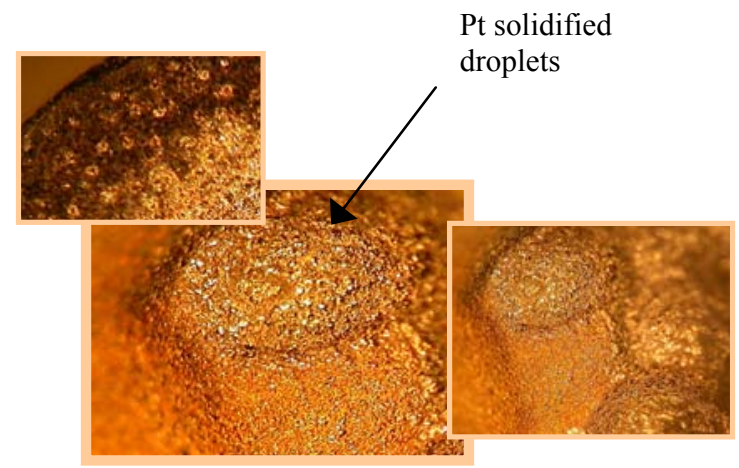

Figure 2.31 Surface of the gold electrode in $[\mathrm{Au}(+) \mathrm{Pt}(-)]$ system 
We observed electrode surface damage for both electrodes however more severe for the cathode. It is known that positive ions have a larger mass and transfer more energy to the respective electrode than electrons. Another effect supporting the asymmetry can be expansion of the plasma diameter at the anode surface, causing a decrease in the local heat flux (Barrufet et al., 1991).

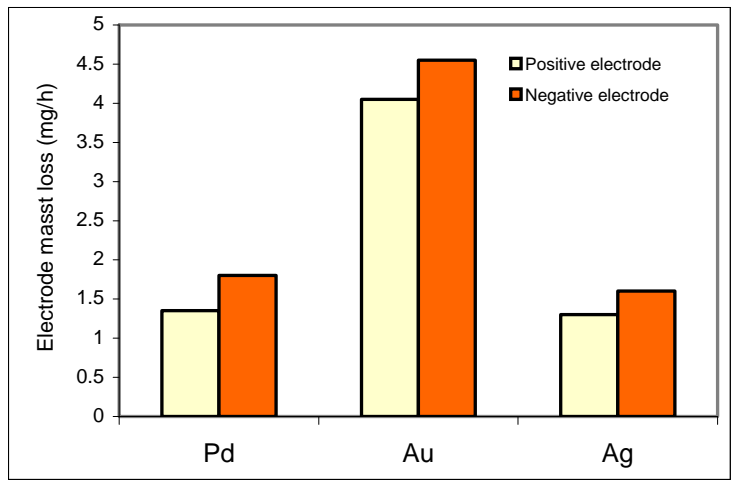

Figure 2.32 Electrode mass losses for $\mathrm{Au}, \mathrm{Ag}$ and $\mathrm{Pd}$ $(C=20 \mathrm{nF}, d=1 \mathrm{~mm}, f=120 \mathrm{~Hz}, Q=1 \mathrm{~L} / \mathrm{min}, \mathrm{Ar})$

\subsubsection{Aerogel Formation}

During the synthesis of carbon aerosol at very small or zero flow, web-like assemblies with a very low density and high porosity were formed inside the generator. This self-assembled aerogel is depicted in Figure 2.33.a. Chain-like agglomerates around $12 \mathrm{~mm}$ in length bridged the positive electrode to the grounded housing.

To simulate the electric field inside the generator using the Maxwell's equations, COMSOL (3.3) was utilized. Figure 2.34 shows the electric field lines, confirming that the particles are assembled along the field lines bridging the positive electrode to the grounded housing. It is likely that particles of both polarities contribute to the formation of these assemblies. This has been proposed in connection with similar observations in a laser ablation generator (El-Shall et al., 2003). Neutral particles may also be involved in the formation of the assemblies due to dielectrophoresis. Chain aggregates become polarized in the electric field, and if the field is non-uniform a dipole force results.

The aerogel remained stable for a long time inside the generator but partially collapsed when removed, as in the previous work (Schleicher et al., 1995). The fragile nature indicates rather weak inter-particle binding forces. Web-like assemblies, were also observed for Pd (Figure 2.33.b), Au and some other metallic nanoparticles. Due to the high surface area and porosity, such structures may find applications in the field of catalysis, if stabilization is possible.

In order to study the morphology of the assemblies, samples were taken from different regions for electron microscopy. The micrographs are shown in Figure 2.35. The web like assemblies contained carbon particles of a few nanometers in diameters and some tube-like structures with interlayer spacing of around $0.342 \mathrm{~nm}$ were found at the tip of the electrode, where the peak electric field was about $2 \times 10^{4} \mathrm{Vcm}^{-1}$. According to the quasi-liquid tip model for growth of multi-wall nano tubes (Sugimoto, 2000), carbon neutrals $\left(\mathrm{C}, \mathrm{C}_{2}\right)$ and ions $\left(\mathrm{C}^{+}\right)$ deposit on the cathode and since the temperature is very high, they may go through a liquefied state. The electrostatic force (Maxwell tension) exerted on liquid-like particles pulls the surface towards the direction of the field and the tip collects vapour and ions. These strings elongate as long as the field is sustained and carbon vapour and ions are supplied (Sugimoto, 2000). Thus the short length of the structures in Figure 2.35 may be due to the short spark life. 


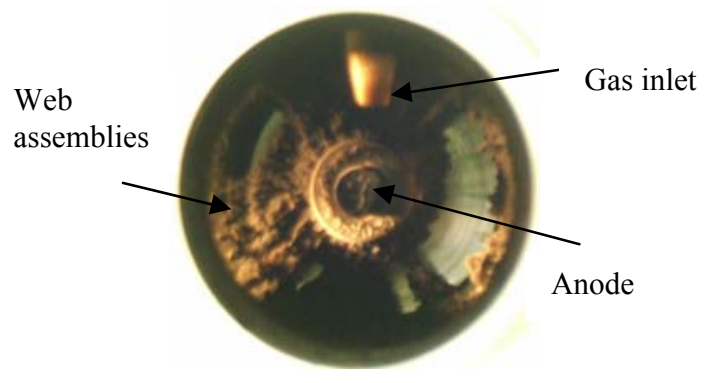

(a)

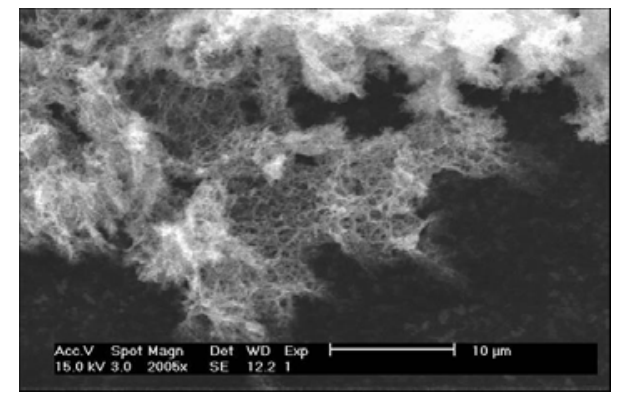

(b)

Figure 2.33 (a) Self-assembly of carbon nanoparticles (plane perpendicular to the electrode), (b) SEM micrograph of $P d$ web assembly (scale bar represents $10 \mu \mathrm{m}$ )

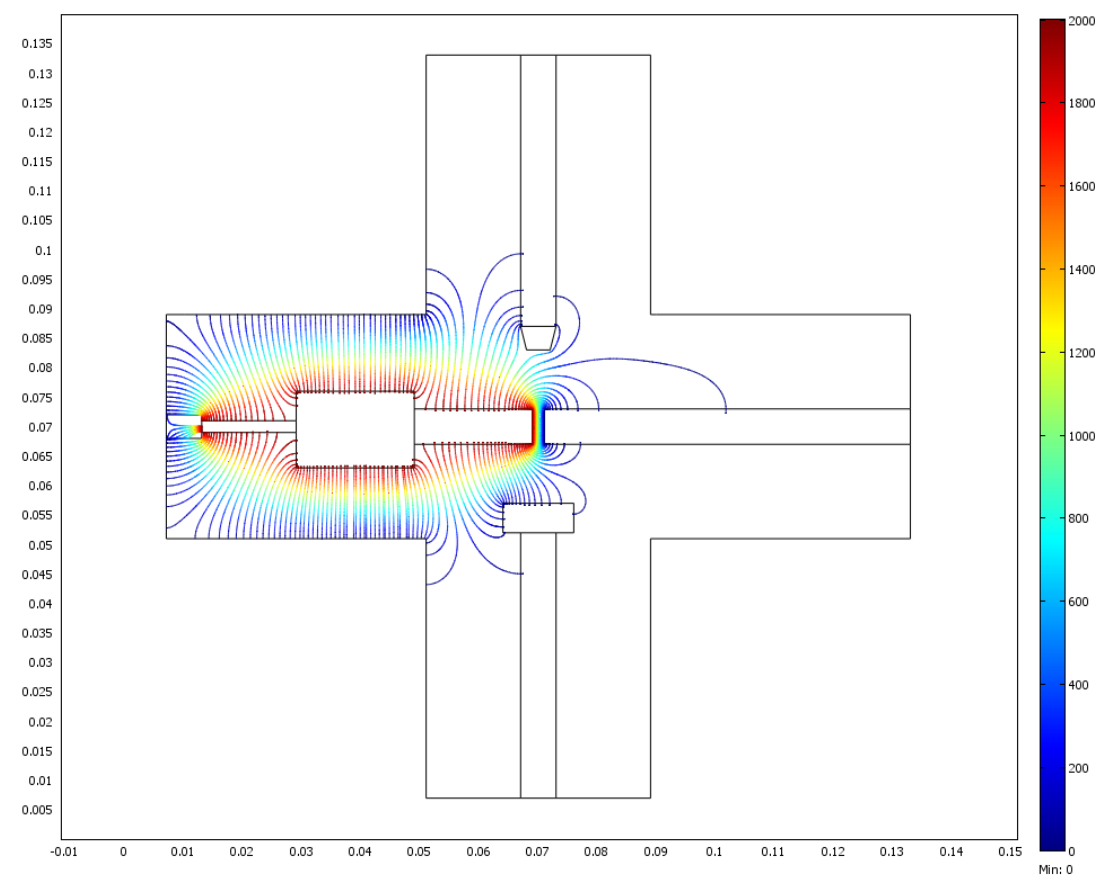

Figure 2.34 Electric field inside the generator, simulated by COMSOL (3.3)
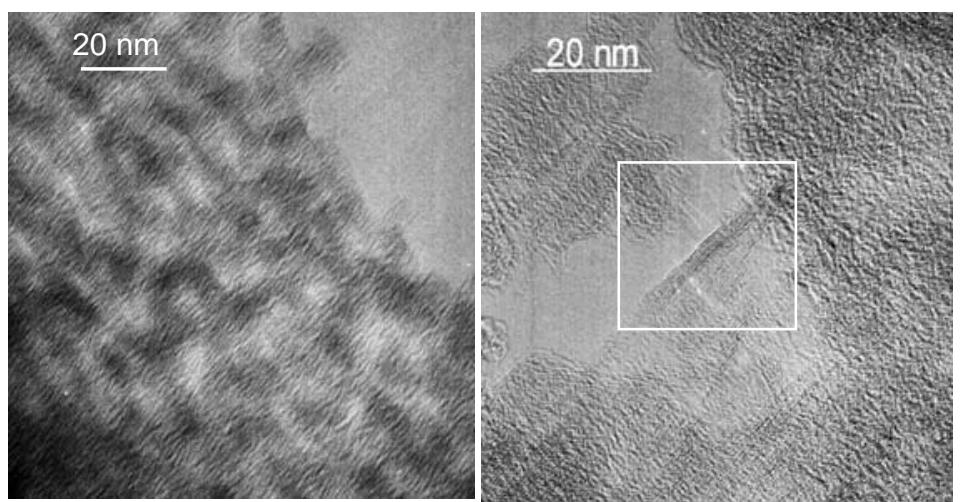

Figure 2.35 TEM micrographs of carbon nanostructures 


\subsection{Conclusion}

The production of nanoparticles by microsecond spark discharge evaporation has systematically been studied. The method is of special interest, because it is continuous, clean, extremely flexible with respect to material and the mean particle size can be controlled via the energy per spark, which is in turn determined through variable parameters. In contrast to laser ablation, which has comparable advantages, spark generators require only electric power and could be scaled up to produce arbitrary nanoparticulate material quantities by using a large number of electrodes. Separated, unagglomerated particles a few nanometers in size can be obtained, if the inert gas flow through the generator is high enough with respect to the spark repetition frequency. In principle, this enables coating of individual particles, if a vapour deposition step is added. The nanoparticulate mass produced is typically $5 \mathrm{~g} / \mathrm{kWh}^{4}$.

On the example of gold we showed that with gases purified at the spot, the method produces particles that are so clean that sintering of agglomerated particles occurs at room temperature, leading to branched solid structures. The influence of a number of parameters on the primary particle size and mass production rate has been studied and qualitatively understood. Due to the fast and very local evaporation of material, high vapour loading is achieved for a short time, which makes the model of Lehtinen and Zachariah (2002), applicable. Our findings agree with qualitative predictions of this model, according to which the particle growth process under high vapour loading conditions is strongly influenced by particle heating due to the release of surface free energy in coalescence. The model allows qualitative predictions regarding particle size, when the energy per spark and the gas pressure are varied. When the gas flow rate was sufficiently high, the particle size became independent of the flow rate, indicating that the primary particle production process is shorter than a millisecond and that this process is not influenced by turbulent dilution, which takes place when particle growth is practically completed. At slow flow the gas flow rate does have an influence on the final particle size, but this is due to slow coagulation at room temperature after primary particle formation. Different electrode materials led to different mean primary particle sizes and mass production rates, where thermal conductivity, evaporation enthalpy and the boiling point had a major influence.

\section{References}

Barrufet M. A. , M. R. Patel, P. T. Eubank, Novel Computations of a Moving Boundary Heat Conduction Problem Applied to EDM Technology, Computers Chem. Engng, Volume 15, No. 8, 1991, pp. 609-618

Borra Jean- Pascal, Nucleation and Aerosol Processing in Atmospheric Pressure Electrical Discharges: Powders Production, Coatings and Filtration, J. Phys. D: Appl. Phys., Volume 39, 2006, R19-R54

Buffat P., Borel, J. P., Size Effect on the Melting Temperature of Gold Particles, Physical Review A, Volume 13, 1976,2287

Cundall C. M., J. D. Craggs, Electrode Vapor Jets in Spark Discharges, Spectrochimica Acta, Volume 7,1955, pp. 149-164

\footnotetext{
${ }^{4}$ As a comparison, milling $1 \mathrm{~kg}$ of Yttrium stabilized Zirconia (YSZ) to $100 \mathrm{~nm}$ requires a minimum energy of $100 \mathrm{kWh}$ with an efficiency of $10 \%$ for the ball mill (Osterwalder et al., 2006).
} 
El-Shall M. Samy, Victor Abdelsayed, Yezdi B. Pithawalla, and Edreese Alsharaeh, Vapor Phase Growth and Assembly of Metallic, Carbon, and Silicon Nanoparticle Filaments, J. Phys. Chem. B, Volume107, 2003, pp. $2882-2886$

Evans D. E., The Generation and Characterization of Metallic and Mixed Element Aerosols for Human Challenge Studies, Aerosol Science and Technology, Volume 37, 2003, pp. 975-987

Evans Douglas E., Roy M. Harrison, Jon G. Ayres, the Generation and Characterization of Elemental Carbon Aerosols for Human Challenge Studies, Journal of Aerosol Science, Volume 34, 2003, pp. 1023-1041

Fridman Alexander, Lawrence A. Kennedy, Plasma Physics and Engineering, 2004, Taylor \& Francis Books, Inc.

Fuchs N. A., On the Stationary Charge Distribution on Aerosol Particles in a Bipolar Ionic Atmosphere, Pure and Applied Geophysics, Volume 56, No. 1, 1963, pp. 185-193

Gleiter H., J. Weissmuller, O. Wollersheim, R. Wurschum, Nanocrystalline Materials: a Way to Solids with Tunable Electronic Structures and Properties?, Acta Materialia, Volume 49, 2001, pp. 737-745

Helsper C. , W. M $\ddot{o}$ lter, Investigation of a New Aerosol Generator for the Production of Carbon Aggregate Particles, Atmospheric Environment, Volume 27A, No. 8, 1993, pp 1271-1275

Hemsalech G. A., de Watteville, M. C. C. R. Hebd, Seance Acad. Sci, Volume 144, 1907, p. 1338

Hesu M. S., M. A. Meyers, A. Berkowitz, Synthesis of Nanocrystalline Titanium Carbide by Spark Erosion, Scripta Metallurgica et Materialia, Volume 32, Number 6, 1995, pp. 805-808

Hinds William C., Aerosol Technology, Properties, Behaviors, and Measurements of Airborne Particles, John Wiley\&Sons, 1999

Hinot, K., Burtscher, H, . Weber, A. P., and Kasper, G.,the Effect of the Contact between Platinum and Soot Particles on the Catalytic Oxidation of Soot Deposits on a Diesel Particle Filter, Appl. Catalysis BEnvironmental, Volume 71, 2007, pp. 271-278

Horvath H., M. Gangl, a Low-Voltage Spark Generator for Production of Carbon Particles, Journal of Aerosol Science, Volume 34, 2003, pp. 1581-1588

Kim Jong-Taek, Jen-Shih Chang, Generation of Metal Oxide Aerosol Particles by a Pulsed Spark Discharge Technique, Journal of Electrostatics, Volume 63, 2005, pp. 911-916

Krylovt V., on the Theory of Surface Acoustic Wave Generation by Electric Spark Discharge, Journal of Physics D: Applied Physics, Volume 25, 1992, pp. 155-161

Lehtinen Kari E. J., Ulrika Backman, Jorma K. Jokiniemi, Makku Kulmala, Three-Body Collisions as a Particle Formation Mechanism in Silver Nanoparticle Synthesis, Journal of Colloid and Interface Science, Volume 274, 2004, pp. 526-530

Lehtinen Kari E.J., Michael R. Zachariah, Energy Accumulation in Nanoparticle Collision and Coalescence Processes, Journal of Aerosol Science., Volume 33, 2002, pp. 357-368

Llewellyn Jones F., Electrode Erosion by Spark Discharges, British Journal of Applied Physics, Volume 1, 1950, pp. 60-65

Mäkelä Jyrki M., Pasi Aalto, Boris Z. Gorbunov, Pekka Korhonen, Size Distributions from Aerosol Spark Generator, Journal of Aerosol Science, Volume 23, Supplement 1, 1992, pp. S233-S236

Maly R., M. Vogel, Initiation and Propagation of Flame Fronts in Lean $\mathrm{CH}_{4}$-Air Mixtures by the Three Modes of the Ignition Spark, Symposium (international) on Combustion, Volume 17, Number 1, 1979, pp. 821-831 
Meek J. M., J. D. Craggs, Electrical Breakdown of Gases, Oxford at the Clarendon Press, 1953

Naidu M. S., V. Kamaraju, High Voltage Engineering, Mcgraw Hill, 1995

Oh Hyuncheol, Junho Ji, Jaehee Jung, Sangsoo Kim, Synthesis of Titania Nanoparticles via Spark Discharge Method Using Air as a Carrier, Materials Science Forum, Volume 544-545, 2007, pp. 143-146

Osterwalder N., C. Capello, K. Hungerbuhler, W. J. Stark, Energy Consumption during Nanoparticle Production: How Economic Is Dry Synthesis, Journal of Nanoparticle Research, Volume 8, 2006, pp. 1-9

Perry Robert H., Perry’s Chemical Engineers’ Handbook, McGraw-Hill, 1984

Reinmann R., M. Akram, Temporal Investigation of a Fast Spark Discharge in Chemically Inert Gases, J. Phys. D: Appl.Phys., Volume 30, 1997, pp. 1125-113

Roth C., G. A. Ferron , E. Karg, B. Lentner, G. Schumann, S. Takenaka, J. Heyder, Generation of Ultrafine Particles by Spark Discharging, Journal of Aerosol Science and Technology, Volume 38, 2004, pp. 228-235

Schleicher B., S. Friedlander, Fabrication of Aerogel-Like Structures by Agglomeration of Aerosol Particles in an Electric Field, J. Colloid and Interface Science, 1995,Volume 180, pp. 15-21

Schwyn S., E. Garwin, A. Schmidt-Ott, Aerosol Generation by Spark Discharge, Journal of Aerosol Science, Volume 19, Number 5, 1988, pp. 639-642

Seipenbusch M., A. P. Weber, A. Schiel, G. Kasper, Influence of the Gas Atmosphere on Restructuring and Sintering Kinetics of Nickel and Platinum Aerosol Nanoparticle Agglomerate, Journal of Aerosol Science, Volume 34, 2003, pp. 1699-1709

Sher E., J. Ben-Yaish, T. Kravchik, 'on the Birth of Spark Channels", Combustion and Flame, Volume 89, 1992, pp. 186-194

Soldera Flavio A., Frank T. Mucklich, Klaus Hrastnik, Thomas Kaiser, Description of the Discharge Process in Spark Plug and its Correlation with the Electrode Erosion Patterns, IEEETransaction on Vehicular Technology, Volume 53, Number 4, 2004, pp. 1257-1265

Soldera F., A. Lasagni, F. Mucklich, T. Kaiser, K. Hrastnik, Determination of the Cathode Erosion and Temperature for the Phases of High Voltage Discharges Using FEM Simulations, Computational Materials Science, Volume 13, Number 5, 2005, pp. 444-453

Sugimoto Tadao, Fine Particles Synthesis, Characterization, and Mechanisms of Growth, Marcel Dekker, 2000

Swihart Mark T., Vapor-Phase Synthesis of Nanoparticles, Current Opinion in Colloid \& Interface Science, Volume 8, Number 1, 2003, pp. 127-133

Szenete R. N., R. J. Munz, M. G. Drouet, Copper-Niobium and Copper-Tungsten Composites as Plasma Torch Cathodes, Journal of Physics D: Applied Physics, Volume 27, 1994, pp. 1443-1447

Tabrizi N. S., M. Ullmann, V. A. Vons, U. Lafont, A. Schmidt-Ott, Generation of Nanoparticles by Spark Discharge, Journal of Nanoparticle Research, Volume 11, Number 2, 2009, pp. 315-332

Tsai Y., T. Masuzawa, an Index to Evaluate the Wear Resistance of the Electrode in Micro-EDM, Journal of Materials Processing Technology, Volume 149, 2004, pp. 304-309

Ullmann M., S. K. Friedlander, A. Schmidt-Ott, Nanoparticles Formation by Laser Ablation, Journal of Nanoparticle Research, Volume 4, 2002, pp. 499-509

Va'vra J., J. A. Maly, P. M. Va'vra, Soft X-ray Production in Spark Discharges in Hydrogen, Nitrogen, Air, Argon and Xenon Gases, Nuclear Instruments and Methods in Physics Research A, Volume 418, 1998, pp. 40541 
Vemury S., S. Pratsinis, Self Preserving Size Distributions of Agglomerates, Journal of Aerosol Science, Volume 26, 1995, pp. 175-185

Watters R.obert L. Jr., James R. DeVoe, Francis H. Shen, John A. Small, Ryna B. Marinenko, Characteristics of Aerosols Produced by the Spark Discharge, Anal. Chem., Volume 61, 1989, pp. 1826-1833

Wiedensohler A., H. J. Fissan, Bipolar Charge Distributions of Aerosol Particles in High-Purity Argon and Nitrogen, Aerosol Science and Technology, Volume 14, 1991, pp. 358-364 


\section{Chapter 3}

\section{Technical Notes on Spark-Generated Particles in View of Applications}

\subsection{Introduction}

Metallic nanoparticles are regarded as a promising class of advanced materials due to their potential applications in chemical, physical, and biochemical fields. The electronic structures of metallic nanoparticles show major deviation from that of the bulk ${ }^{1}$. They have the ability to store excess electrons (Lahiri et al., 2005) and usually present excellent catalytic properties (Sugimoto, 2000). Recent studies show that the Surface Plasmon Resonance (SPR) excited in metallic nanoparticles leads to selective photoabsorption, scattering, and local electromagnetic field enhancement (Hirai and Kumar, 2006).

In this chapter characterization of particles produced from various metals in a wide range of physical and chemical properties provides us with valuable information on sintering and oxidation. Our observation on large particles which are sometimes formed and an explanation of this effect is given. Moreover, the functionality of our particles in specific applications is investigated.

\subsection{Experimental Set-up for Particle Characterization}

For synthesis of monometallic nanoparticles, a pair of identical electrodes was always applied in the spark generator. All the electrodes were of high purity $(>99.99 \%)$. They were rods of 3-6.35 mm diameters with flat ends. The carrier gas was argon (with $99.999 \%$ purity). For particle collection purposes, the gas flow rate was adjusted to $0.8-1.5 \mathrm{~L} / \mathrm{min}$. The interelectrode distance was kept around $1 \mathrm{~mm}$ and the capacitance was usually $20 \mathrm{nF}$. The schematic of the experimental set-up is shown in Figure 2.5.

In order to study the size, morphology, and structure of the particles, transmission (TEM) and scanning (SEM) electron microscopy were performed on the samples. The transmission electron microscope was a Philips $\mathrm{CM} 30 \mathrm{~T}$ with a $\mathrm{LaB}_{6}$ filament as the source of electrons operated at $300 \mathrm{kV}$.

For the determination of the specific surface areas of the particles $\mathrm{N}_{2}$ adsorption isotherms were measured on a Quantachrome Autosorb-6B.

\footnotetext{
1 The electronic structure of a metal particle critically depends on its size. For small particles, due to confinement of the electron wavefunction, the electronic states are not continuous, but discrete. The average spacing of successive quantum levels, $\delta$, known as the Kubo gap, is given by $\delta=4 \mathrm{E}_{\mathrm{f}} / 3 \mathrm{n}$, where $\mathrm{E}_{\mathrm{f}}$ is the Fermi energy of the bulk metal and $\mathrm{n}$ is the number of valence electrons in the nanoparticle (Rao et al., 2000). At room temperature $\mathrm{kT} \approx 25 \mathrm{meV}$. For $\mathrm{kT}>\delta$ the particle is expected to behave metallic.
} 


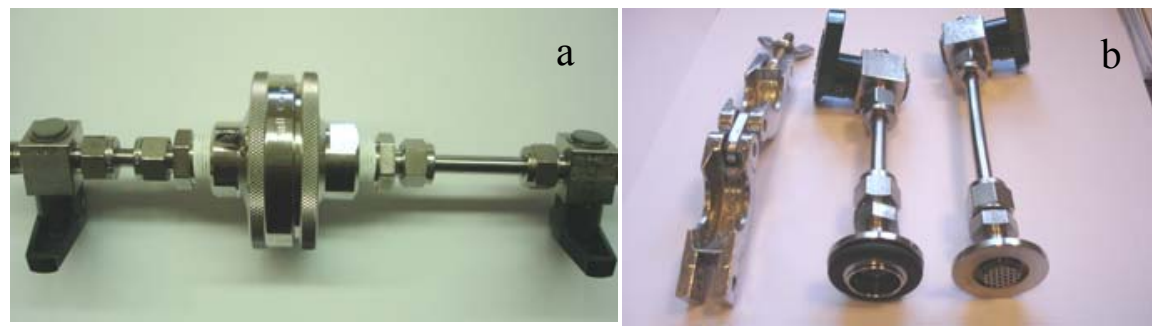

Figure 3.1 Particle collector (a), and TEM sampler (b)

Particles were collected on a membrane filter (Millipore, Durapore) placed inside a stainless steel, airtight sampler (Pall), shown in Figure 3.1.a. For electron microscopy, particles were collected on a carbon coated $3 \mathrm{~mm}$ nickel or copper 200 mesh grid which was attached to a coarse grid (Figure 3.1.b), fixed in a holder and mounted perpendicular to the aerosol flow. In order to protect the particles from oxidation, these samplers could be isolated from the system by two valves at both ends.

\subsection{Particle Characterization Results and Discussion}

Electron Microscopy -Transmission electron micrographs of $\mathrm{Au}, \mathrm{Ag}, \mathrm{Pd}$ and $\mathrm{Pt}$ nanoparticles produced by the spark generator are shown in Figure 3.2. The gold particles show more pronounced sintering than the other metals. Only the isolated particles remain spherical and the contacting ones have sintered, forming irregular shapes. In very small particles most of the atoms are located on the surface. Surface atoms are less restricted in their ability to vibrate than those in interior, and they are able to make larger excursions from their equilibrium positions. At higher temperatures these fluctuations can cause a breakdown in the symmetry of the nanoparticle, resulting in the formation of a liquid-like droplet of atoms (Poole, 2003). The fact that small metal particles are liquid-like or at least have mobile atoms on their surfaces explains sintering of the collected particles or of particles that have collided in the gas phase at room temperature. Evidently, this also happens if only one partner is small, as the inset in the Au particle micrograph of Figure 3.2 shows. The irregular $\mathrm{Au}$ aggregates seem to become stable when areas of extreme surface curvature are eliminated. The fact that room temperature sintering is only observed for Au particles can be explained by the higher inertness of these particles. The other particles may be stabilized by a monolayer of chemisorbed gas impurities, namely oxygen. Au, Ag, Pd, Pt are noble metals in bulk form, but their nobility on the nanoscale is uncertain. Compared to the bulk, they have an increased chemical reactivity due to the enhanced surface area and the reduced number of nearest neighbours on a curved surface. Since $\mathrm{Au}_{2} \mathrm{O}_{3}, \mathrm{AgO}$ and $\mathrm{PtO}_{3}$ decompose at 150, 100, and $25^{\circ} \mathrm{C}$ respectively, it is unlikely that these oxides occur during particle formation process and we assume that any oxide is formed later.

Formation of a thin layer of contamination (for example oxide) around the particles due to chemisorption of impurities in the system may have a significant effect on the aggregation of the particles. The surface layer may prevent a pure metal-metal contact, decrease the surface energy and slow down the sintering kinetics. 

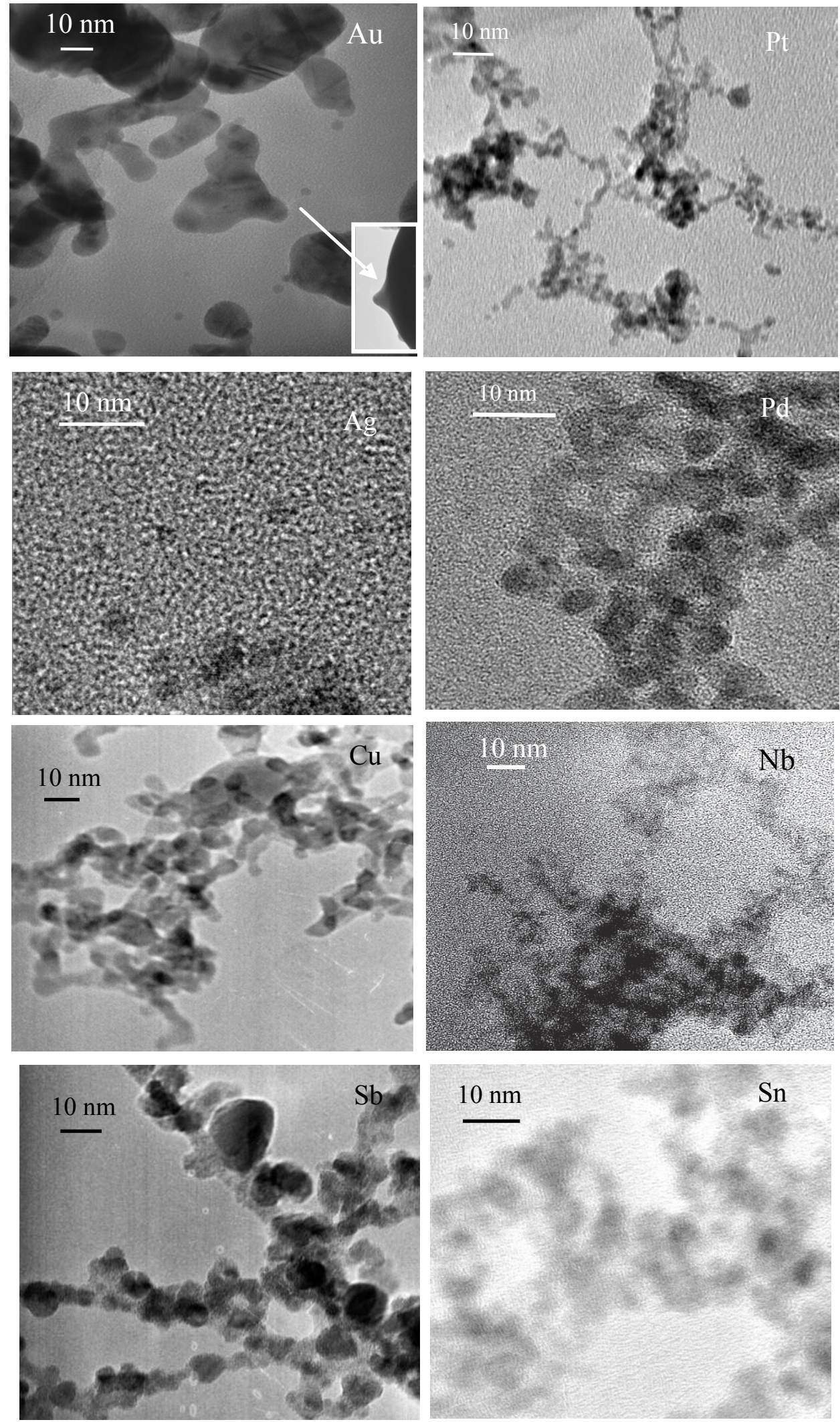

Figure 3.2 Electron micrographs of $A u, P t, A g, P d, C u, N b, S b, S n^{2}$

\footnotetext{
${ }^{2} \mathrm{Cu}, \mathrm{Sb}$, and $\mathrm{Sn}$ particles were produced in a joint work with Lafont et al. (2009).
} 
In a sintering process ${ }^{3}$, diffusion of material through the contact points results in the formation of neck between particles with a driving force of reducing the surface area and thus surface free energy of the system (Ashby, 1974). Among various mechanisms ${ }^{4}$ contributing to the sintering of bulk crystalline particles only surface diffusion ${ }^{5}$ and grain boundary diffusion make a remarkable contribution to nanoscale sintering. In addition, mechanical rotation, plastic deformation via dislocation generation and transmission and amorphization of sub-critical grains play a major role in the early stages of nanoscale sintering (Zeng et al., 1998). Thus sintering kinetics depends strongly on the type of metal observed. Apart from this, the absence of an oxide layer and the metal-metal contact is a prerequisite for sintering. Depending on the surface purity of the particles and properties of the material, various morphologies and different levels of neck formation occur.

Our observations from TEM analysis on different metals show that spark generated particles are mainly nano sized. The size distribution of the primary particles depends on the material however particles are usually in the size range of 2-15 $\mathrm{nm}$ suggesting that the main mechanism of particle formation in spark discharge generator is evaporation-condensation (Appendix C). Beside these, few large particles are often present along with the huge number of nanoparticles. These are referred to below in the discussion of BET measurements and in Sec. 3.3.1. Different structures and textures are produced. For example, noble metallic particles are all crystalline and $\mathrm{Nb}$ particles are mainly in the amorphous phase. An amorphous solid will be formed if the cooling rate is faster than the rate at which atoms (molecules) can organize into a more thermodynamically favorable crystalline state.

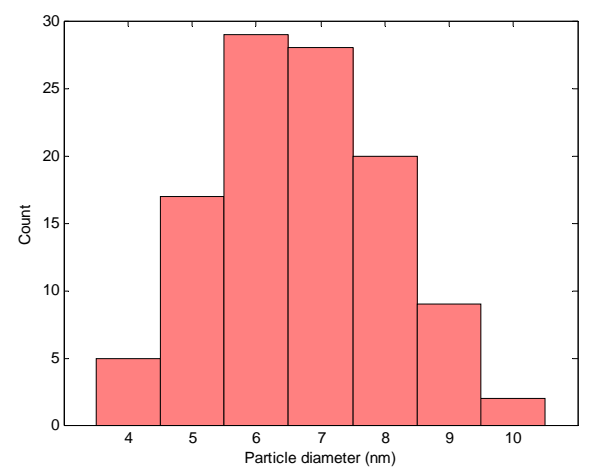

Figure 3.3 A typical size histogram of metallic nanoparticles generated by the spark discharge

A typical size histogram of primary particles produced by the spark generator, obtained from TEM analysis is shown in Figure 3.3. It has a geometric diameter of $6.5 \mathrm{~nm}$ and a geometric standard deviation of 1.23 (Appendix C). Most size distributions observed can be approximated by a lognormal function. For a lognormal count distribution, $95 \%$ of the particles fall within a size range of $\exp \left(\ln C M D \pm 2 \ln \sigma_{g}\right)$ which goes from $C M D / \sigma_{g}^{2}$ to $C M D \times \sigma_{g}^{2}$. CMD and $\sigma_{\mathrm{g}}$ stand for Count Median Diameter ${ }^{6}$ and geometric standard deviation, respectively. For a majority of applications $\sigma_{\mathrm{g}}<1.2$ is a sufficiently narrow size distribution (Hinds, 1999).

\footnotetext{
${ }^{3}$ In this text, sintering should be distinguished from coalescence in which two small particles colide and form a larger spherical particle whereas the former means aggregation and attachment of particles by neck formation.

${ }^{4}$ Surface diffusion, lattice diffusion from the surface, vapor transport, grain boundary diffusion, lattice diffusion from the grain boundary, and diffusion through dislocations (Zeng et al., 1998).

${ }^{5}$ The driving force for surface diffusion is the gradient of the chemical potential along the surface (Wu et al., 1993)

${ }^{6}$ The particle size for which half of the total number of particles are larger and half are smaller.
} 

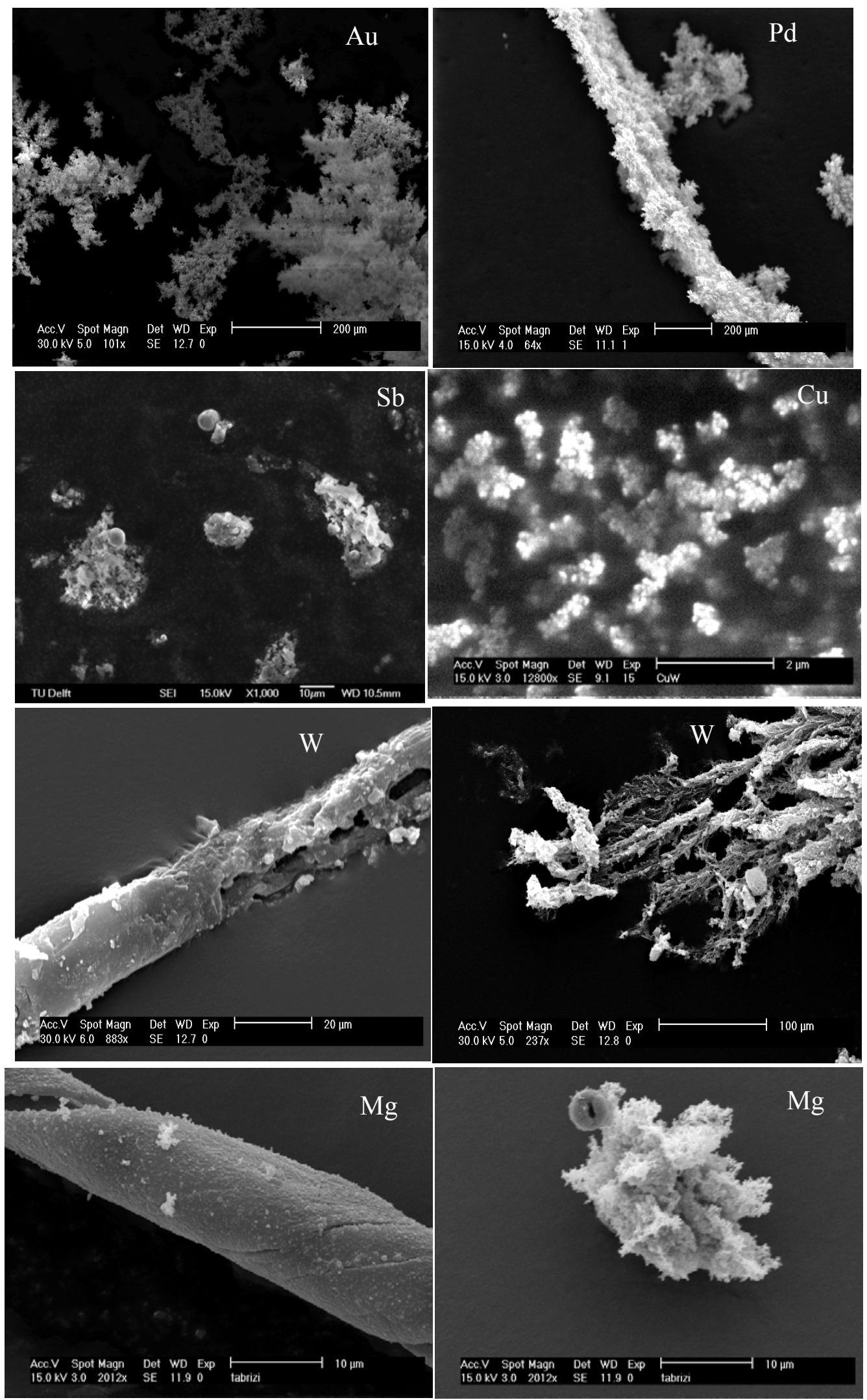

Figure 3.4 SEM images of $A u, P d, S b, C u, W, M g$ 
Scanning electron microscopy (SEM) with a much lower magnification than TEM confirms that the primary particles are mainly in the nano-scale and few large spherical particles are also present (for example see $\mathrm{Sb}$ ). The morphologies of the particle assemblies differ from material to material (for example coral, thread, and grape bunch shapes). Even for the same material various forms of assemblies can be obtained (Figure 3.4).

In self-assembly processes the affinity of the particles to the substrate as well as repulsive or attractive interaction between the particles plays an important role. Moreover, depending on the magnetic and electronic properties of the nanoparticles, an external magnetic or electric field can affect the structure of the assemblies. An electric field is present in the spark generator and may favor alignment of particles to form linear assemblies. Dynamics of the flow can also play a role.

BET Measurements -The high surface area of nanoparticles with respect to their mass is one important reason for the great interest in them from various fields including catalysis and hydrogen storage. In the present study the $\mathrm{N}_{2}$ adsorption isotherms at $77 \mathrm{~K}$ have been measured to derive the specific surface area $\left(\mathrm{m}^{2} \mathrm{~g}^{-1}\right)$, using the Brunauer-Emmet-Teller theory (Rouquerol et al., 1999). Presuming a perfectly spherical (shape factor 6), non-porous particle, the corresponding particle diameter can be calculated by:

Particle diameter $=\frac{\text { shape factor }}{\text { surface area } * \text { metal density }}$

Table 3.1 Specific surface areas and calculated BET diameters of particles produced from different electrodes

\begin{tabular}{|l|l|l|l|}
\hline $\begin{array}{l}\text { Electrode } \\
\text { material }\end{array}$ & $\begin{array}{l}\text { BET surface area } \\
\left(\mathrm{m}^{2} \mathrm{~g}^{-1}\right)\end{array}$ & $\begin{array}{l}\text { Calculated BET } \\
\text { diameter }(\mathrm{nm})\end{array}$ & $\begin{array}{l}\text { Mean diameter of } \\
\text { particles by TEM }(\mathrm{nm})\end{array}$ \\
\hline $\mathrm{Cu}$ & 130 & 5.1 & 5 \\
\hline $\mathrm{W}$ & 72 & 4.3 & 4 \\
\hline $\mathrm{Au}$ & 14 & 22 & 5 \\
\hline $\mathrm{Sb}$ & 65 & 14 & 10 \\
\hline $\mathrm{Mg}$ & 42 & 39 & 4 \\
\hline $\mathrm{Pd}$ & 58 & 9 & 4 \\
\hline $\mathrm{Ag}$ & $<10$ & $>57$ & 4 \\
\hline $\mathrm{Pt}$ & $<10$ & $>28$ & 3 \\
\hline
\end{tabular}

For a number of electrode materials, the specific BET surface areas of spark-generated particles and the corresponding diameters are listed in Table 3.1, where the BET diameters are compared with the averaged diameters from the TEM analysis. For $\mathrm{Cu}$ and $\mathrm{W}$ these diameters agree well. Discrepancies of factors of 2 to 15 on the other hand are observed for noble metals and $\mathrm{Mg}$.

This is in agreement with the statement made above that oxidation hinders sintering. There is a larger number of particle-particle contacts in the BET sample than in the TEM micrograph probes. This qualitatively explains the discrepancy between the two diameters for the noble metals. Another possible explanation is the formation of a small fraction of large particles as observed in some of the cases. Large, unagglomerated, round particles were observed by electron microscopy (for example Figure 3.5). It suggests that parallel to the evaporationcondensation process, particle formation by solidified droplets emitted from the locally heated electrode surface may occur. 

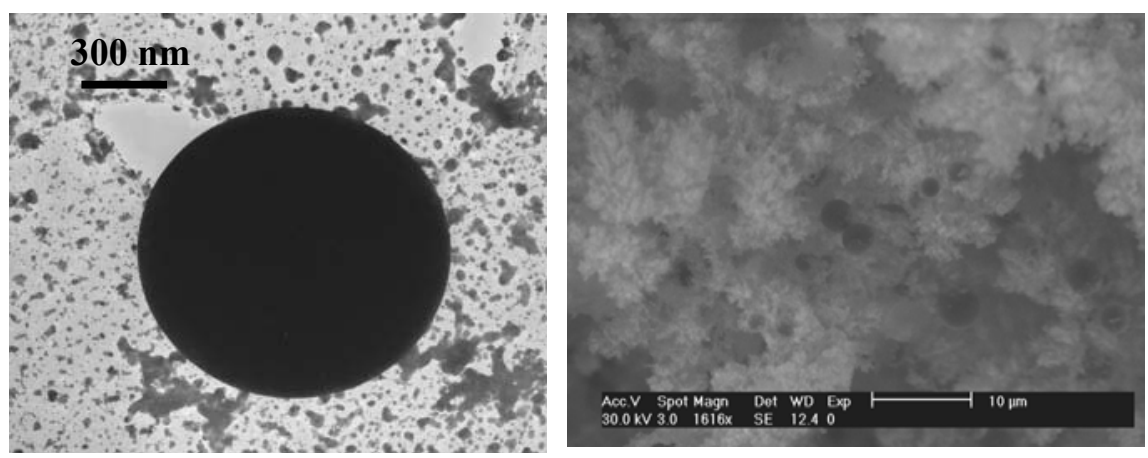

Figure 3.5 Electron micrographs displaying sputtered large particles of Au

The size of these particles ranges from tens of nanometers to a few microns. They may form a substantial part of the total mass, and this may (partly) explain the large values determined for some BET diameters above.

\subsubsection{Explanation of Large Particle Formation}

In the breakdown phase of a spark discharge a conductive filament is formed between the electrodes which leads to a hot arc discharge where the energy transferred to the electrodes can form deep craters.

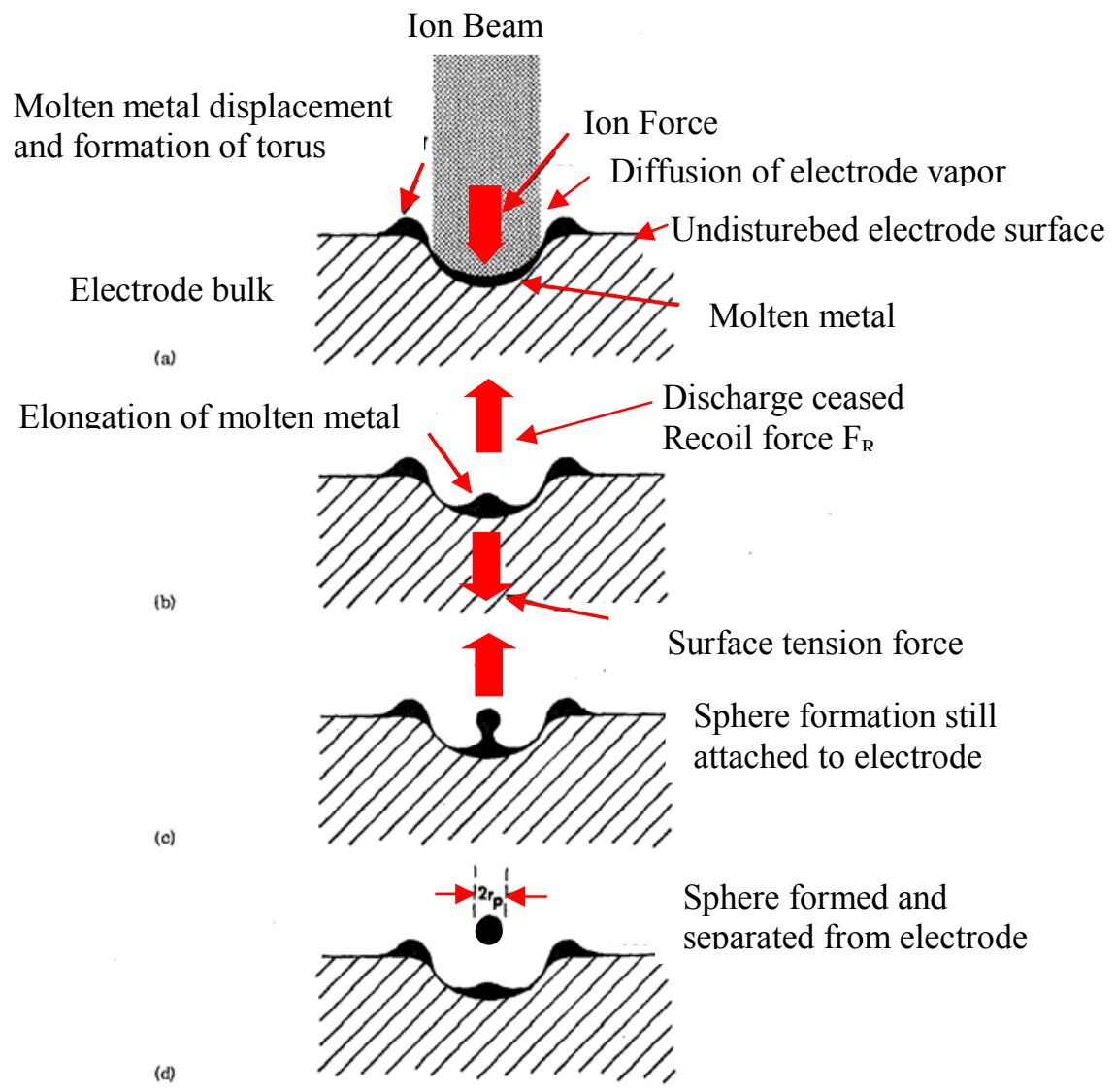

Figure 3.6 Formation mechanism of large particle from electrode surface (adopted from Gray et al., 1974) 
These are formed by Joule heating and ion impact heating causing local melting of the electrodes. Ejection of molten metal from these microscopic pools is possible under the action of electric and/or acoustic fields (Petr et al., 1980), plasma pressure and the pressure created by ion bombardment. According to the erosion model proposed by Gray et al. (1974), ion bombardment creates a force, acting on molten metal. When the discharge ceases, the unbalanced recoil force is directed outwards from the electrode. Since the surface tension of the molten metal acts against this recoil force, it is of great importance. If the recoil force exceeds the surface tension force, the molten droplet is sputtered. Otherwise it oscillates in the pool and solidifies (Figure 3.6).

The large solidified droplets are usually not detected in samples which are collected by diffusion. In our sampling method, particles are captured not only by diffusion but also by interception.

In a further study on the large particles, two different electrodes, one made of pure $\mathrm{Cu}$ and the other one made of pure $\mathrm{W}$ were utilized to produce particles. Figure 3.7 shows two micrographs containing such big particles whose compositions are shown in Table 3.2.
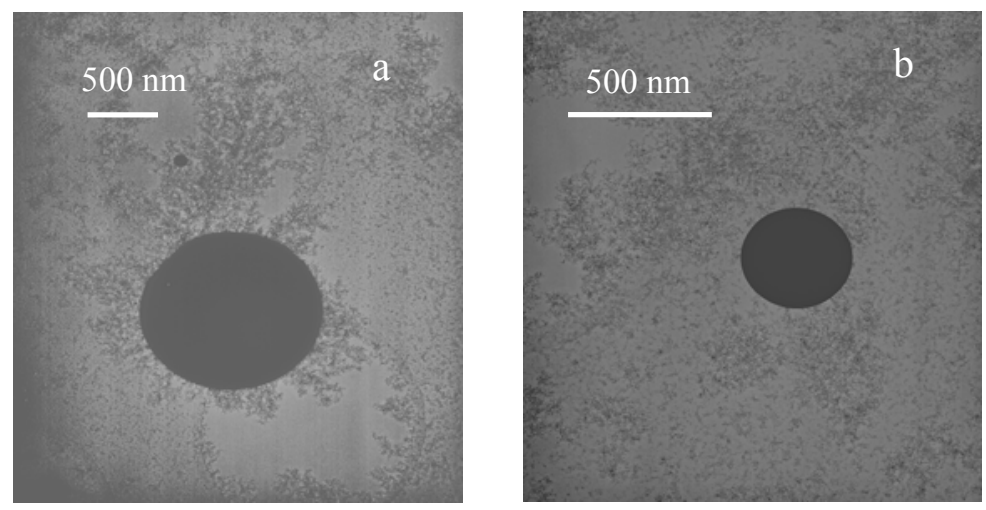

Figure 3.7 Cu-W particles produced from compositionally different electrodes of $C u$ and $W$

The EDS elemental analysis shows that the big spheres are almost pure $\mathrm{Cu}(1280 \mathrm{~nm})$ or pure $\mathrm{W}(375 \mathrm{~nm})$. This supports the hypothesis that they are solidified droplets ejected from the molten metal formed at the tip of the electrodes due to interaction with energetic plasma. If the particles were formed by vapour condensation, they would be mixed (see chapter 5).

Table 3.2 EDS elemental analysis of the big particles

\begin{tabular}{|l|l|l|l|}
\hline Figure (3.7.a) & $\mathrm{W}$ & $\mathrm{Cu}$ & $\mathrm{O}$ \\
\hline Large sphere $(1280 \mathrm{~nm})$ & 0.61 & 97.63 & 1.76 \\
\hline Figure (3.7.b) & & & \\
\hline Large sphere $(375 \mathrm{~nm})$ & 98.37 & 0.64 & 0.99 \\
\hline
\end{tabular}

\subsubsection{Approaches to Suppress Large Particles in the Product}

The large particles produced from solidified droplets are undesired in some applications. Means of eliminating them have therefore been briefly studied below.

Impactor- In order to separate the big particles from the small ones, an impactor typically used ahead of TSI DMAs was applied (Appendix B). However, even for the smallest orifice 
size of these types of impactors $(0.0457 \mathrm{~cm})$ the cutoff size was still too big $(0.338 \mu \mathrm{m})$ and smaller solidified melt particles were transmitted. The induced pressure drop was around 61.2 $\mathrm{cm} \mathrm{H}_{2} \mathrm{O}$ at $1 \mathrm{~L} / \mathrm{min}$ aerosol flow. The captured big particles, piled up on the impaction plate were eventually detached from the surface and re-suspended in the flow. This made the setup unpracticable for continuous use.

To achieve a smaller cut off diameter, a micro-orifice impactor with large number of chemically etched nozzles could be a feasible alternative.

Electrostatic precipitation- To separate the small Pd particles from the large ones, the flowing aerosol was subjected to an electric field between two parallel plates and perpendicular to the flow. The electric force acting on the charged particles causes a fraction of particles to deposit on the plates (Figure 3.8). The voltage between the plates was set to $1000 \mathrm{~V}$, the distance between the plates was $1 \mathrm{~cm}$, and the flow rate was kept $1 \mathrm{~L} / \mathrm{min}$. The dimensions of the plates were $3 \times 8 \mathrm{~cm}$ and designed such that particles with a single elementary charge and smaller than $60 \mathrm{~nm}$ to be precipitated (Appendix B).

As can be seen in Figure 3.8, deposition of the charged particles on the plates illustrated the flow pattern inside the precipitator (from bottom-to-top of the figure), indicating a vortex around the outlet. Particles were mainly deposited on the centerline of the plates along the flow trajectory. Since the particles were deposited on the both plates, we conclude that the spark discharge generates bipolar charged particles. The remarkable amount of deposits on the plates (for example in two hours) indicates that a noticeable fraction of the total particles are charged. To examine the captured particles, four samples from different regions on both plates were collected for SEM analysis.

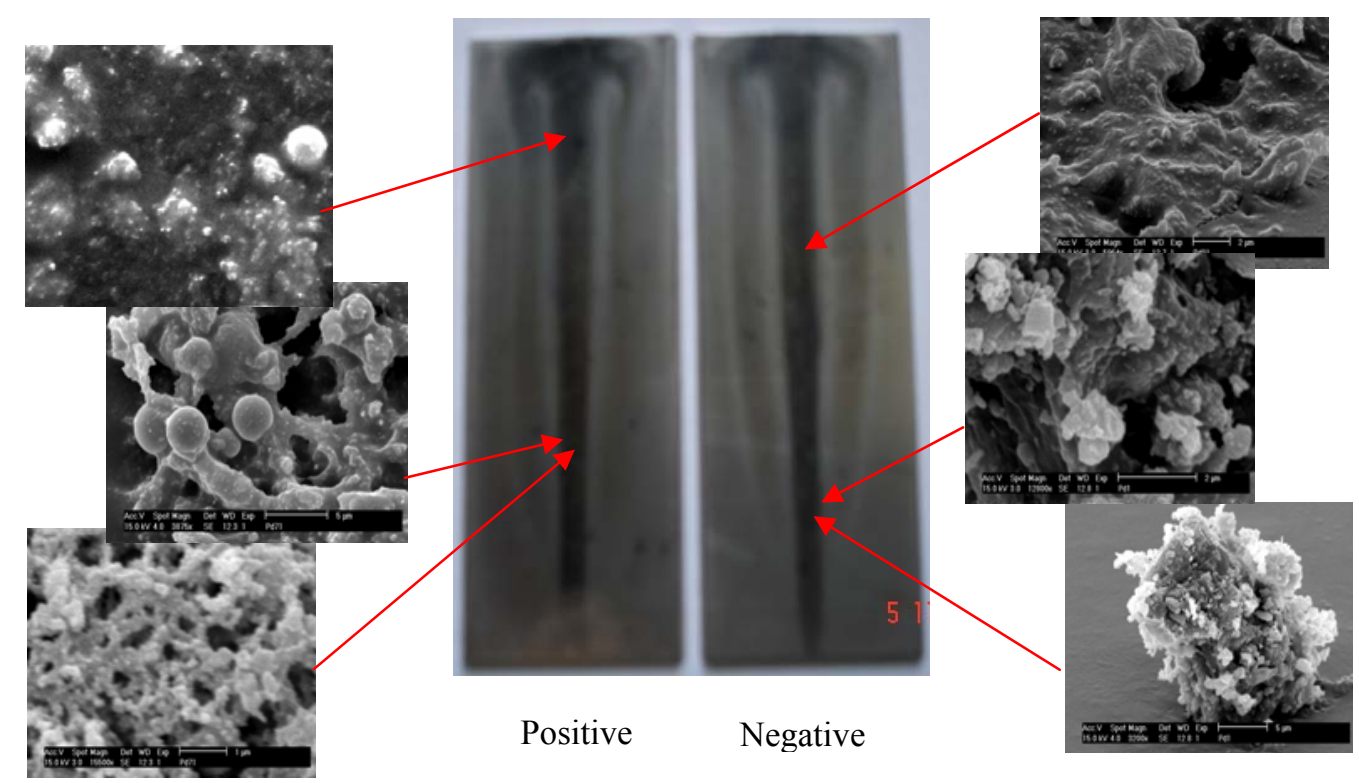

Figure 3.8 Deposition of Pd particles on the precipitator plates and SEM images of the particles

It was observed that in addition to nanoparticles, big particles up to around $3 \mu \mathrm{m}$ were also deposited on the positive plate, indicating that they were negatively charged. These particles must have many elementary charges which enhance their electrical mobility. The images show that the small particles are strongly agglomerated, presumably on the surface and influenced by the action of the electric field. 
Hollow Electrode- According to the above-mentioned erosion model, the big particles come from molten metal pools. So rapid and efficient cooling of the electrode surface should suppress their formation. Thus, with the idea of enhancing heat transfer between the carrier gas and the electrodes, a hole of $3 \mathrm{~mm}$ diameter was drilled into a magnesium electrode of 6 $\mathrm{mm}$ diameter and the hollow electrode (Figure 3.9.a) was used as the inert gas inlet (or outlet). By increasing argon flow rates up to $30 \mathrm{~L} / \mathrm{min}$, no instability was observed in the spark and its frequency (Figure 3.9.b, c). The hollow electrode proved to be operational. The produced particles were sampled and examined under a TEM. The images are shown in Figure 3.10.a, b. Only agglomerates containing nano particles are observable. A dense feather-like structure can be seen in Figure 3.10.a, evidently consisting of nano-sized particles. The solidified melt particles would manifest themselves by a higher contrast. In this case the hollow electrode was used as the gas inlet. In Figure 3.10.b, the hollow electrode was used as the gas outlet. As seen in the image there are some low contrast stains. They are unlikely to be solidified melt entrained from the electrode surface, since their dimensions are quite small and their contrast too low. To understand these structures more study would be required.
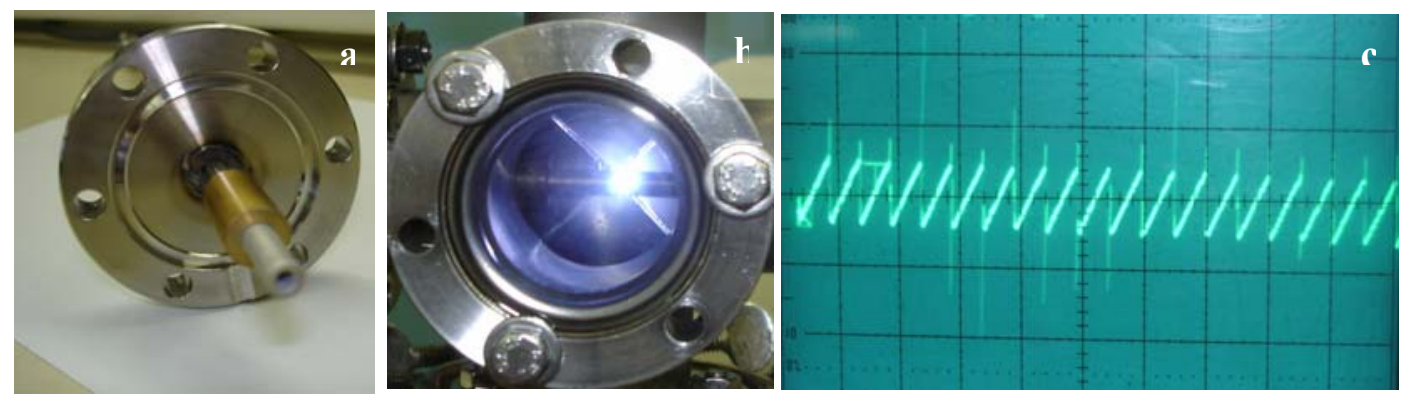

Figure 3.9 Magnesium hollow electrode (a), spark with the hollow electrode (b), spark frequency at 30 $L / \min (c)$
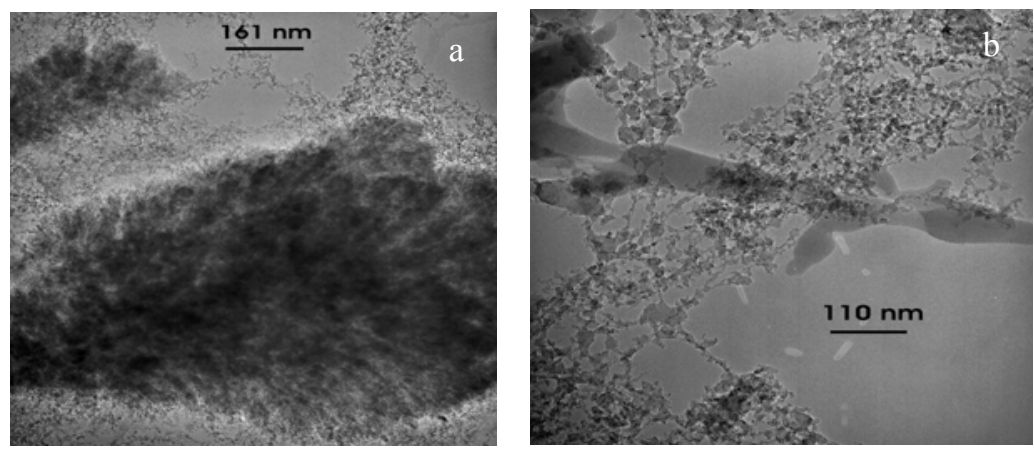

Figure 3.10 Mg particles using hollow electrode as gas inlet (a), and gas outlet (b)

\subsubsection{Notes on Oxidation Behavior}

Due to the high reactivity of most metal nanoparticles with some gaseous species like oxygen, the composition of the processing atmosphere should be controlled. In the case that a thin passive layer on the particle surface protects the particle from further reaction, a low concentration of the reactive gas can be beneficial (Nalwa, 2003; Karmhag et al., 2001; Sanchez-Lopez et al., 1998; Soon Kwon et al., 2004). 
An important criterion to predict the formation of a protective oxide is the Pilling-Bedworth ratio, defined as the ratio of the molecular volume of the oxide to the atomic volume of the metal from which the oxide is formed. If this ratio is less than one as in the case of magnesium $\left(\mathrm{V}_{\mathrm{MgO}} / \mathrm{V}_{\mathrm{Mg}}=0.81\right)$, the oxide grows under internal stress leading to a discontinuous, porous film possessing low protective properties (Kutz, 2002).

According to the theory of oxidation, when more than one type of oxides co-exist with the metal in the system, a multilayer scale forms on the metal, consisting of oxides of varying oxygen content, from metal-rich oxides with a low oxygen equilibrium pressure to oxygenrich oxides with a high oxygen equilibrium pressure (Xu et al., 2007).

The oxide film growth on a metal is limited by transport of ions and charge-compensating electrons through the oxide layer, assisted by a built in electric field (Niklasson et al., 2003). Oxidation of nickel, for example, takes place by metal cation diffusion through a vacancy mechanism, predominately in grain boundaries and other linear defects. When the oxide layer is thick, linear ionic diffusion prevails, a parabolic law is obtained and the square of the oxide thickness is proportional to time. For thin oxide layers, the ion transport is assisted by the electric field due to the difference in electron concentration between the boundaries of the oxide layer (Niklasson et al., 2003). For instance, with a built in potential of 0.1-1 V, the electric field for a $10 \mathrm{~nm}$ oxide thickness is $10^{5}-10^{6} \mathrm{~V} / \mathrm{cm}$. Therefore, additional charges on a particle, for example obtained in the synthesis process, may play a role in the oxidation process.

Figure 3.11.a shows Mg particles produced and analyzed under the inert gas. Although the inert gas was of high purity (Ar 99.999\%), a small amount of oxygen in the system was apparently sufficient to oxidize the particles. The oxide layer around the large particle is clearly distinguishable. Metallic magnesium has a hexagonal close-packed (hcp) structure as seen in the core of the particle. When the sample was exposed to air for X-ray diffraction (XRD) analysis, the sample converted entirely to oxide form as there are no Bragg reflections corresponding to the pure $\mathrm{Mg}$ metal (Figure 3.11.b).
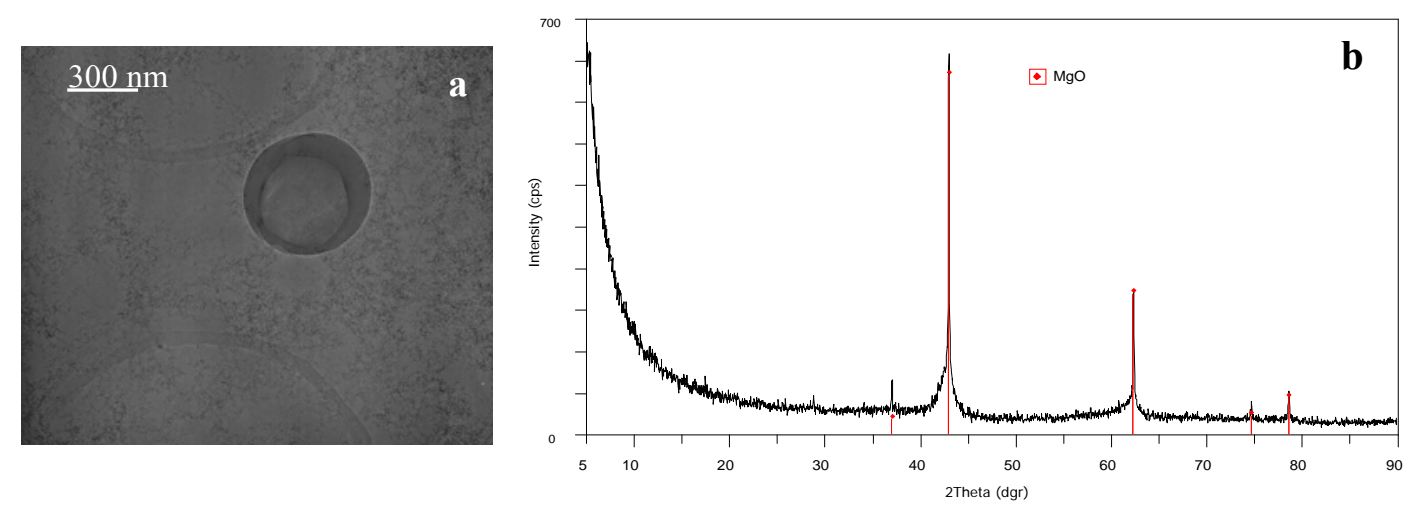

Figure 3.11 TEM micrograph of Mg particles (a), XRD pattern of Mg sample exposed to air showing only oxide phase (b)

A simple model can be used to calculate the percentage of $\mathrm{Mg}$ in particles with various sizes when the oxide layer grows (Figure 3.12). It implies that when the particles are very small (a few nanometers), formation of even a thin layer of oxide means that they are almost fully oxidized.

In order to avoid oxidation of reactive particles, we evacuated the system by a vacuum pump and baked it by a heating tape. Furthermore the carrier gas was purified by moisture and oxygen traps for getting rid of traces of oxygen and humidity and attaining a higher gas purity. Since the particles themselves consume the oxygen of the system, they were also utilized as oxygen getters by being deposited on the internal surfaces. 


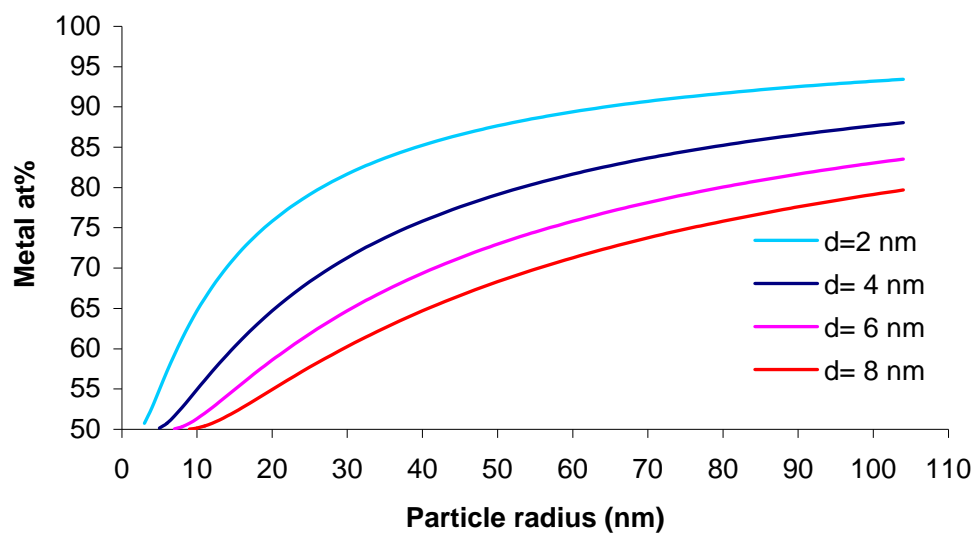

Figure 3.12 Mg atomic percent vs particle size at different oxide layer thicknesses (d)

Additionally some fresh particles were deposited on a spare filter to capture the residual oxygen and humidity of the system (sampler 1 in Figure 3.13a). Furthermore, in the synthesis process a certain amount of the pretreated carrier gas was circulated in a closed loop by an air-tight and oil-free pump. After running the experiments for a sufficient time to clean the system, the main sample was collected on a separate sampler in the closed loop (sampler 2 in Figure 3.13.a).

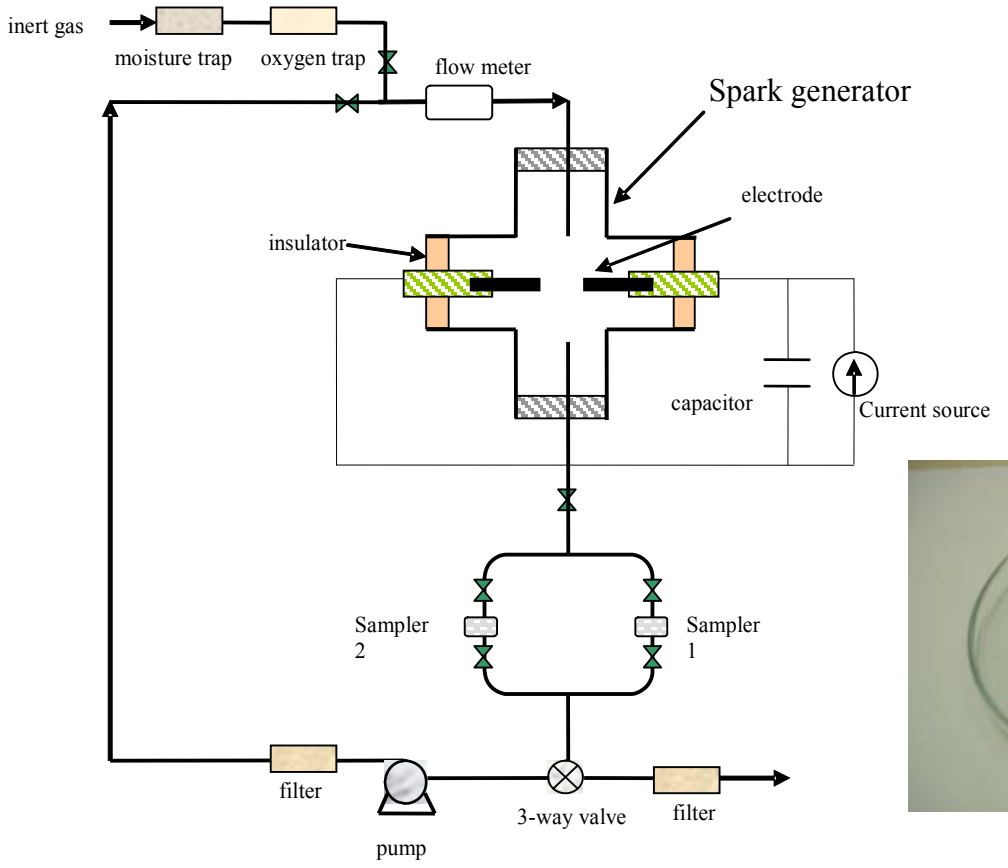

(a)

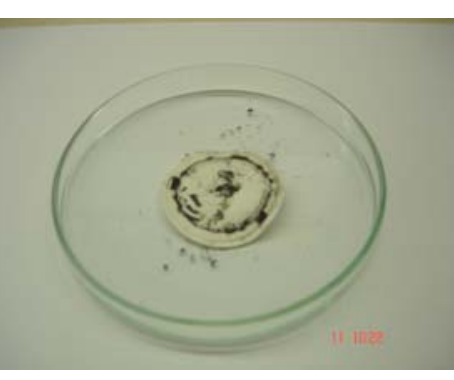

(b)

Figure 3.13 Closed loop for producing unoxidized particles (a), burned Sb particles upon exposure to air (b)

The above mentioned set-up proved to be capable of generating clean particles. Antimony particles produced in this set-up burned upon sudden exposure to air (Figure 3.13.b). This indicates the absence of an initial oxide layer on the surface of particles. 


\subsection{Examples of Applications}

Degradation of particles during synthesis, collection and handling processes can severely affect the applicability of particles. Moreover, application conditions like high temperature which may lead to sintering of particles, etc..., can influence the particle behavior. In this section we briefly test the performance of our particles in the following applications.

\subsubsection{Li-Ion Battery}

Lithium-ion batteries are rechargable batteries containing an intercalation anode material. Lithium ions move from the anode to the cathode during discharge and from the cathode to the anode when charging (Figure 3.14). They exhibit good energy-to-weight ratios, no memory effect, and a slow loss of charge when not in use. The functional components of a lithium ion battery are the anode, cathode, and electrolyte, for which a variety of materials may be used.

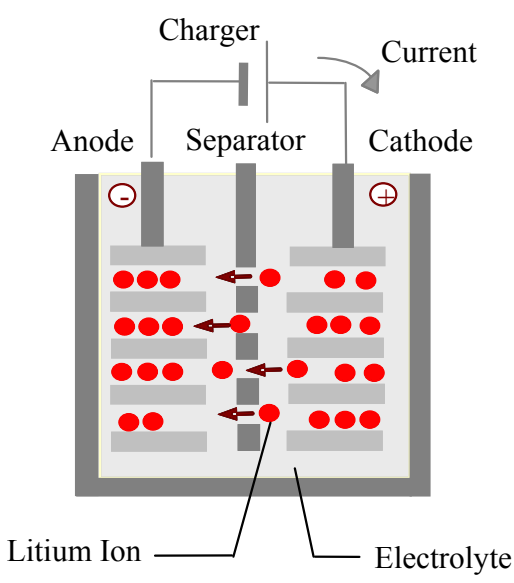

(a)

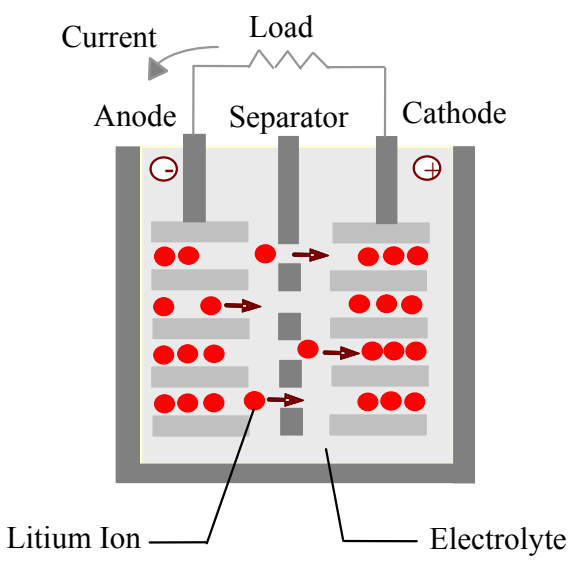

(b)

Figure 3.14 Lithium ion batteries charging (a), and discharging (b), (reproduced from NEC TOKIN Co.)

The most common material for the anode is graphite. The cathode is generally a layered oxide (Dailly et al., 2002), a polyanion, or a spinel. Liquid electrolytes in Li-ion batteries consist of solid lithium-salt electrolytes and organic solvents. Depending on the choice of material for the anode, cathode, and electrolyte the voltage, capacity, life, and safety of a lithium ion battery can change dramatically.

In order to improve the safety as well as the capacity and rate capability of Li-ion batteries, new anode materials are of interest. Li can alloy with elements like $\mathrm{Sb}, \mathrm{Sn}, \mathrm{Al}$, and $\mathrm{Si}$ yielding higher theoretical specific capacities than that obtained for graphite. However, formation of alloy often results in volume expansion occurring in lithiation and as a consequence a poor cycle performance (Bryngelsson et al., 2007). To overcome this problem, one approach can be the use of nanoparticles due to their small absolute volume variation and superplasticity (Li et al., 2002). Buffering the active metal by a matrix or a layer of passive material may also be of interest (Simonin et al., 2007). Therefore, different kinds of metallic nanoparticles were produced in this work and their electrochemical behaviors in Li-ion batteries were investigated in the framework of a PhD project, carried out by L. Simonin. For 
instance, by modifying the synthesis ${ }^{7}$ and collection conditions ${ }^{8}$, samples of antimony and antimony oxides with different levels of crystalinity and composition were generated and their behaviors as anode electrodes in Li-ion batteries were investigated. It was observed that the texture of nanoparticles have an important effect on the insertion of Li ion and the matrix (oxide phase) should have proper porosity for lithium to interact with antimony (Simonin et al., 2007). Details on the characteristics of the samples and their electrochemical behavior are published by Simonin et al. (2007).

\subsubsection{Hydrogen storage}

It is known that the primary metal hydrides that meet gravimetric storage density criteria have high enthalpies of formation exceeding the value $\left(-48 \mathrm{~kJ} / \mathrm{mol} \mathrm{H}_{2}\right)$ for a desirable metal hydride (Appendix D). Recent investigations report that the hydrogen storage properties of metals can be improved by increasing the surface activity induced by reduction in particle size. The grain size can affect thermodynamic properties like plateau pressures and phase boundary envelopes as well as the kinetics (Zaluska et al., 1999).

Therefore, the spark discharge technique was utilized in the present work to produce $\mathrm{Mg}$ and Pd nanoparticles, and hydrogen desorption properties of the particles were studied.

\subsubsection{Mg Nanoparticles}

Light metal hydrides show sluggish $\mathrm{H}_{2}$ absorption/ desorption kinetics ${ }^{9}$ and the reaction occurs at elevated temperatures (350-400 C) over a time scale of hours (Zaluska, 1999). The slow sorption kinetics and thermodynamic stability of $\mathrm{MgH}_{2}$ are the main obstacles in practical applications.

The formation enthalpy $\left(\Delta \mathrm{H}_{f}\right)$ of the Magnesium hydride is large and the Born-Haber cycle for the bulk magnesium-hydrogen system is shown in Figure 3.15.

Applying Hess law, $\Delta \mathrm{H}_{f}$ is expressed by:

$$
\Delta \mathrm{H}_{f}=S+\sum I P+D+E A+U_{t o t}
$$

With sublimation energy (S) of $147 \mathrm{~kJ} \mathrm{~mole}^{-1}$, magnesium ionization potential $\left(\sum \mathrm{IP}\right)$ of 2205 $\mathrm{kJ}$ mole ${ }^{-1}$, dissociation enthalpy of hydrogen molecule (D) of $436 \mathrm{~kJ} \mathrm{~mole}^{-1}$, hydrogen electron affinity (EA) of $72.8 \mathrm{~kJ} \mathrm{~mole}^{-1}$ and lattice enthalpy of $2718 \mathrm{~kJ} \mathrm{~mole}^{-1}$, we get:

$$
\Delta \mathrm{H}_{f} \approx-75 \mathrm{~kJ} / \mathrm{mol} \mathrm{H}_{2}
$$

\footnotetext{
${ }^{7}$ For instance, different types of carrier gas $\left(\mathrm{Ar}, \mathrm{N}_{2}\right)$ were applied in the presence/absence of oxygen and moisture traps.

${ }^{8}$ A slow exposure of sample to air mainly led to the formation of a layer of amorphous oxide on the particle surface and rapid exposure to air led to the particle burning and formation of crystalline oxide $\left(\mathrm{Sb}_{2} \mathrm{O}_{3}\right),(\mathrm{Simonin}$ et al., 2007).

${ }^{9}$ Kinetic factors of hydrogenation include: surface activity, oxide layer penetration, diffusion rate of hydrogen, and mobility of metal-hydride interface (Zaluska et al., 1999).
} 
The large value of $\Delta \mathrm{H}_{f}$ indicates that the hydrogen atoms bind strongly with $\mathrm{Mg}$ atoms, so that the magnesium hydride needs to be heated to a very high temperature ${ }^{10}$ in order to release the hydrogen.

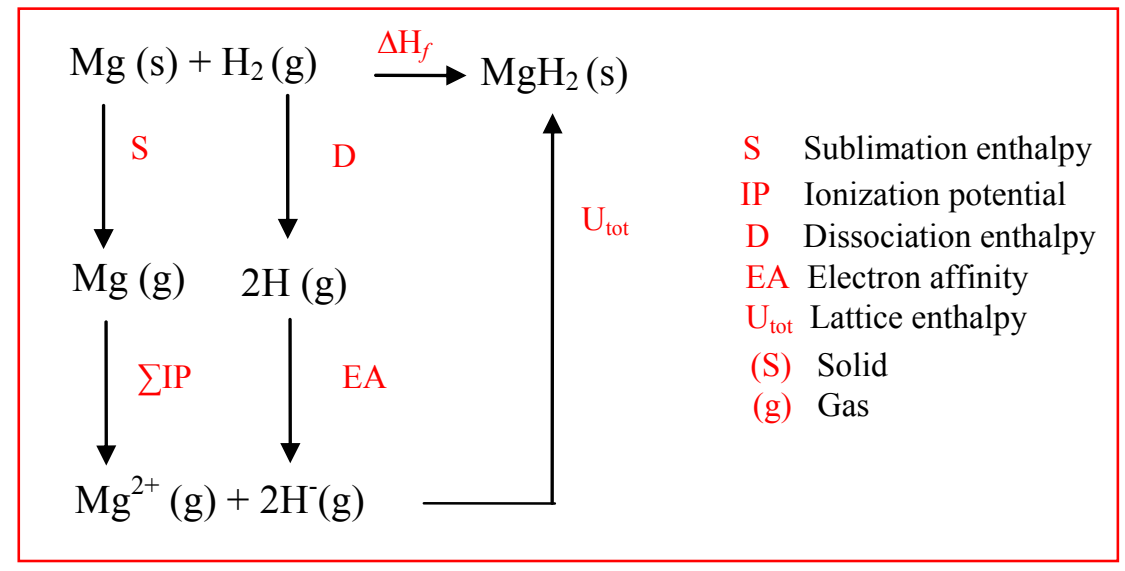

Figure 3.15 Born-Haber cycle for $\mathrm{Mg}-\mathrm{H}_{2}$ system at standard pressure and temperature

Various approaches are reported to destabilize the magnesium hydride ${ }^{11}$ and to reduce $\Delta \mathrm{H}_{f}$ (Liang et al., 2004).

The probability of the adsorption of a $\mathrm{H}_{2}$-molecule on the $\mathrm{Mg}$ surface is $10^{-6}$ (Oelerich et al., 2001) and hydrogen molecules do not readily dissociate at the surface of $\mathrm{Mg}$ to generate hydrogen atoms, thus transition metals having different valances can be utilized to catalyse this process at the surface. Addition of transition metal oxides as catalysts is also reported to improve hydrogen sorption kinetics of $\mathrm{MgH}_{2}$ (Oelerich et al., 2001). The most effective metal and oxide particles are transition metals that are immiscible in $\mathrm{Mg}$ inducing high interfacial energies between the nanoparticles and Mg nanograin boundaries (Yavari, et al., 2003).

Since most metallic nanoparticles have a high affinity to oxygen and a surface layer of oxide can act as a barrier for $\mathrm{H}_{2}$ diffusion, the production process has to be very clean and the collected particles should be handled in an inert atmosphere for example a glove box (Figure 3.16.c). In practice, formation of a thin layer of oxide at the particle surface is usually unavoidable. Therefore the sample often needs annealing which can result in the activation by breakage of the oxide layer due to the difference in the thermal expansion coefficients of pure metal and metal oxide at elevated temperature ${ }^{12}$. Magnesium is sensitive not only to oxygen but also to moisture. The presence of water degrades the oxide film by forming a less stable hydrated oxide ${ }^{13}$.

\footnotetext{
${ }^{10}$ This is about $280{ }^{\circ} \mathrm{C}$ in an environment of 1 bar of $\mathrm{H}_{2}$, or $260{ }^{\circ} \mathrm{C}$ in TDS studies (desorption in a vacuum using a linear temperature ramp rate).

${ }^{11}$ For instance: modification of the $\mathrm{Mg}$ lattice by creating stress and strain, formation of $\mathrm{Mg}$ solid solution, doping $\mathrm{MgH}_{2}$ with other ions, formation of $\mathrm{Mg}$ intermetallic compounds or complex hydrides.

For light metal hydrides the interactions between the pure metal (A) and $\mathrm{H}$ atoms are ionic with transferring a charge from $\mathrm{A}$ to the $\mathrm{H}$ atom and formation a strong bond. In hydrogen storage alloys, there is a covalent interaction between $\mathrm{B}$ element and the hydrogen atoms, caused by the hybridization of the atomic orbitals to form molecular orbitals. By mixing elements $\mathrm{A}$ and $\mathrm{B}$, the $\mathrm{B}-\mathrm{H}$ interaction is enhanced while $\mathrm{A}-\mathrm{H}$ interaction is weakened. This allows for easier hydrogen desorption (Cooper, 2005). For example in the case of $\mathrm{Mg}_{2} \mathrm{Ni}$ relative to the pure $\mathrm{Mg}$, addition of $\mathrm{Ni}$ lowers the reaction enthalpy.

${ }^{12}$ The oxide layer on magnesium surface cracks if the temperature exceeds $400^{\circ} \mathrm{C}$ (Zaluska et al., 1999).

${ }^{13}$ Exposure of bulk Mg to air results in the formation of a layer on the pure metal with 20-50 nm thickness, consisting of $\mathrm{Mg}, \mathrm{MgO}$, and 50-60 wt \% $\mathrm{Mg}(\mathrm{OH})_{2}$. Annealing at temperatures higher than $350^{\circ} \mathrm{C}$, causes magnesium hydroxide to decompose.
} 
The crystal structure of $\mathrm{MgH}_{2}$ is shown in Figure 3.16.a. Nanocrystalline magnesium hydrides are reported to show improved kinetics due to the high fraction of grain boundaries that provide fast diffusion paths for hydrogen and heterogeneous nucleation sites for the hydride phase (Klassen et al., 2001).

In the present work, magnesium nanoparticles of a few nanometers (4-5 nm) in size were produced to study their hydrogen storage behavior (Figure 3.16.b). The black color of the nanoparticulate $\mathrm{Mg}$ sample indicates that the particles are metallic (Figure 3.16.c). Preliminary tests on $\mathrm{H}_{2}$ desorption behaviour were carried out in a thermal desorption spectroscopy (TDS) setup (Figure 3.17.b), which consists of the measurement of the gas released from the sample by a quadrupole mass spectrometer while the temperature is continuously increased with time (Castro et al, 2000). The information obtained from TDS experiments in metal hydride may be difficult to interpret since hydrogen desorption may take place simultaneously with other processes like surface modifications, amorphouscrystalline phase transition or solid state reactions (Fernández and Sanchez, 2003).

Figure 3.18 shows the hydrogen desorption behaviour of magnesium nanoparticles. The particles were charged under 4 and 8 bar of hydrogen pressure at $480^{\circ} \mathrm{K}$ for different periods of time $\left(15 \mathrm{~min}\right.$ to $3 \mathrm{hr}$ ), then the sample was cooled down to $140^{\circ} \mathrm{K}$. The discharge took place in vacuum $\left(0.1 \mathrm{~Pa}\right.$ ) while the temperature ramp was set to $1 \mathrm{~K} \mathrm{~min}^{-1}$ (from $140^{\circ}$ to $700^{\circ}$ $\mathrm{K}$ in $10 \mathrm{hr}$ ). All the curves show the maximum hydrogen release at almost the same temperature around $180^{\circ} \mathrm{C}$. This is quite promising, because this temperature is significantly lower than values reported for larger particles ${ }^{14}$. Wagemans et al. (2005) studied the effect of crystal size on the thermodynamic stability of magnesium and magnesium hydride, using ab initio Hartree-Fock and density function theory calculations. They predicted a desorption temperature of $200^{\circ} \mathrm{C}$ for a crystallite size of $0.9 \mathrm{~nm}\left(\mathrm{Mg}_{9} \mathrm{H}_{18}\right)$. Since their predicted desorption temperature is close to our results, it may infer that the crystal sizes composing our particles are very small or that the particles are quasi amorphous ${ }^{15}$ (Wagemans et al, 2005).

A typical hydrogen storage capacity of the produced $\mathrm{Mg}$ particles was calculated to be around $6.3 \mathrm{wt} . \%$. As $7.6 \mathrm{wt} . \%$ is the theoretical maximum capacity of $\mathrm{MgH}_{2}$, this is also promising.

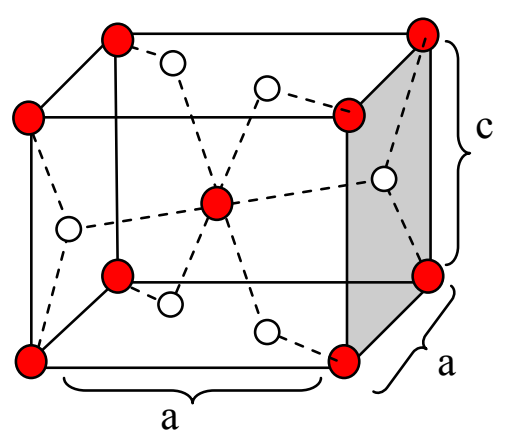

(a)
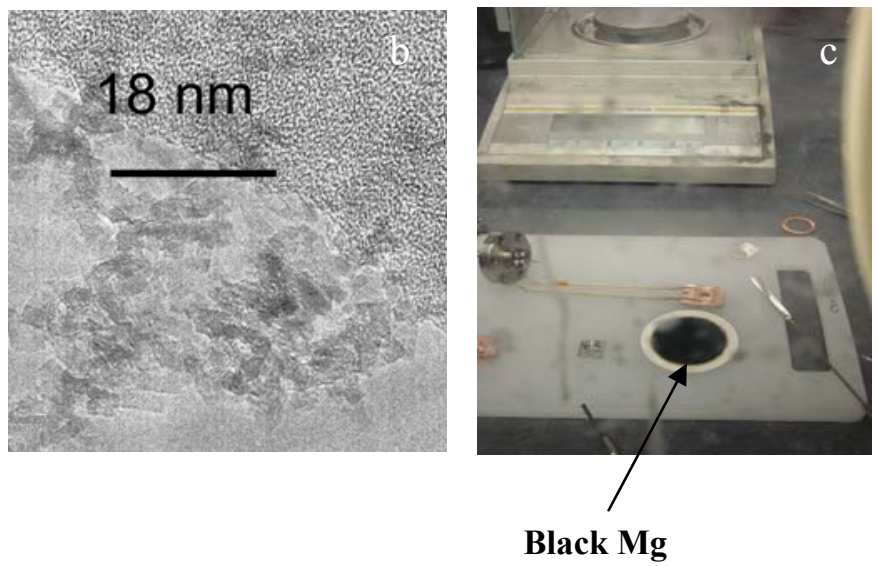

Figure 3.16 Crystal structure of $\mathrm{MgH}_{2}(a)^{16}$, TEM image of $\mathrm{Mg}$ nanoparticles (b), black $\mathrm{Mg}$ deposited on the filter and handled in the glove box (c)

\footnotetext{
${ }^{14}$ For bulk (or ball-milled) $\mathrm{MgH}_{2}$, the TDS spectrum shows a single emission peak at about 530-580 K (Zaluska et al., 1999; Zaluska et al., 2001; RRR Faculty of Applied Science, TUDelft).

${ }^{15}$ Distinction between truly amorphous and crystalline phases is difficult if the size of the crystals is very small.

${ }^{16}$ The powder diffraction file JCPDS 12-0697 lists $\mathrm{a}=0.4517 \mathrm{~nm}$ and $\mathrm{c}=0.30205 \mathrm{~nm}$.
} 

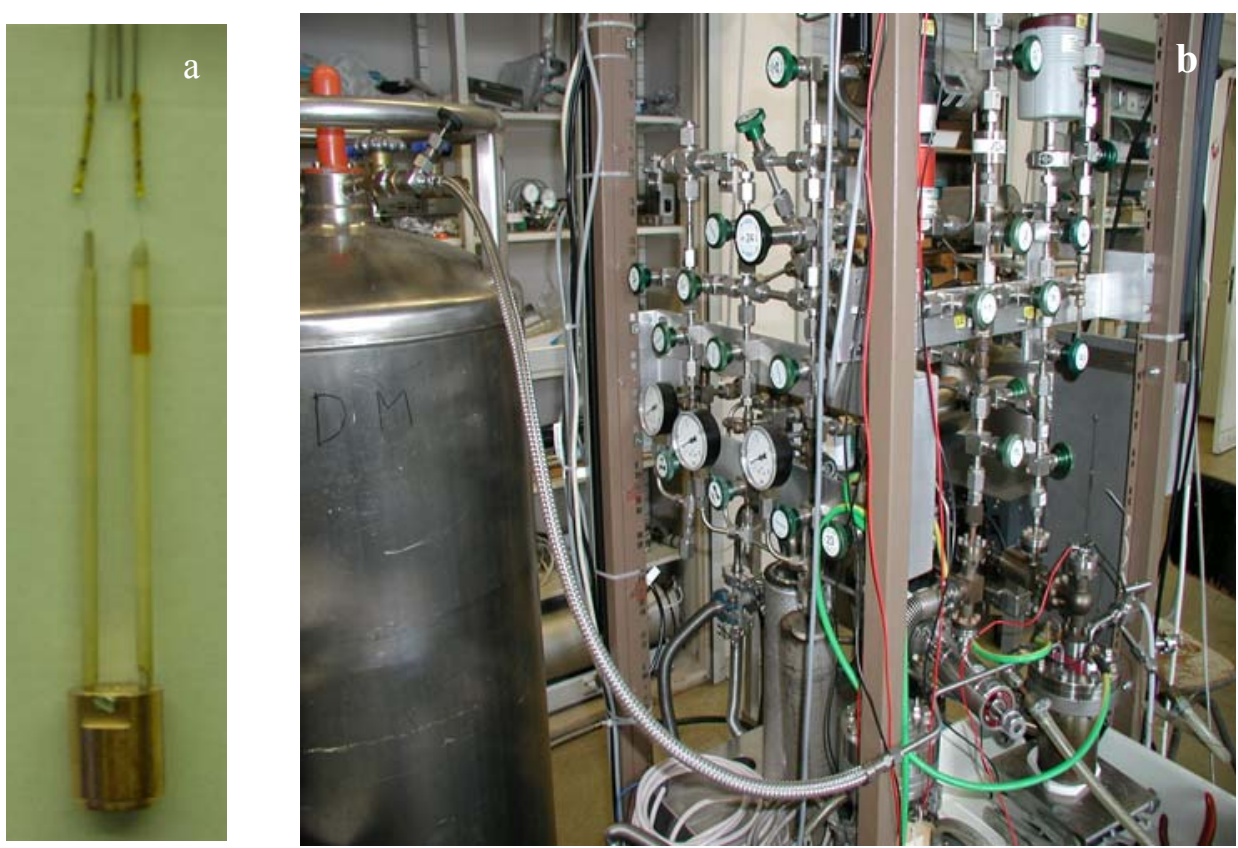

Figure 3.17 Sample holder (a), thermal deposition setup for hydrogen desorption measurements $(b)^{17}$

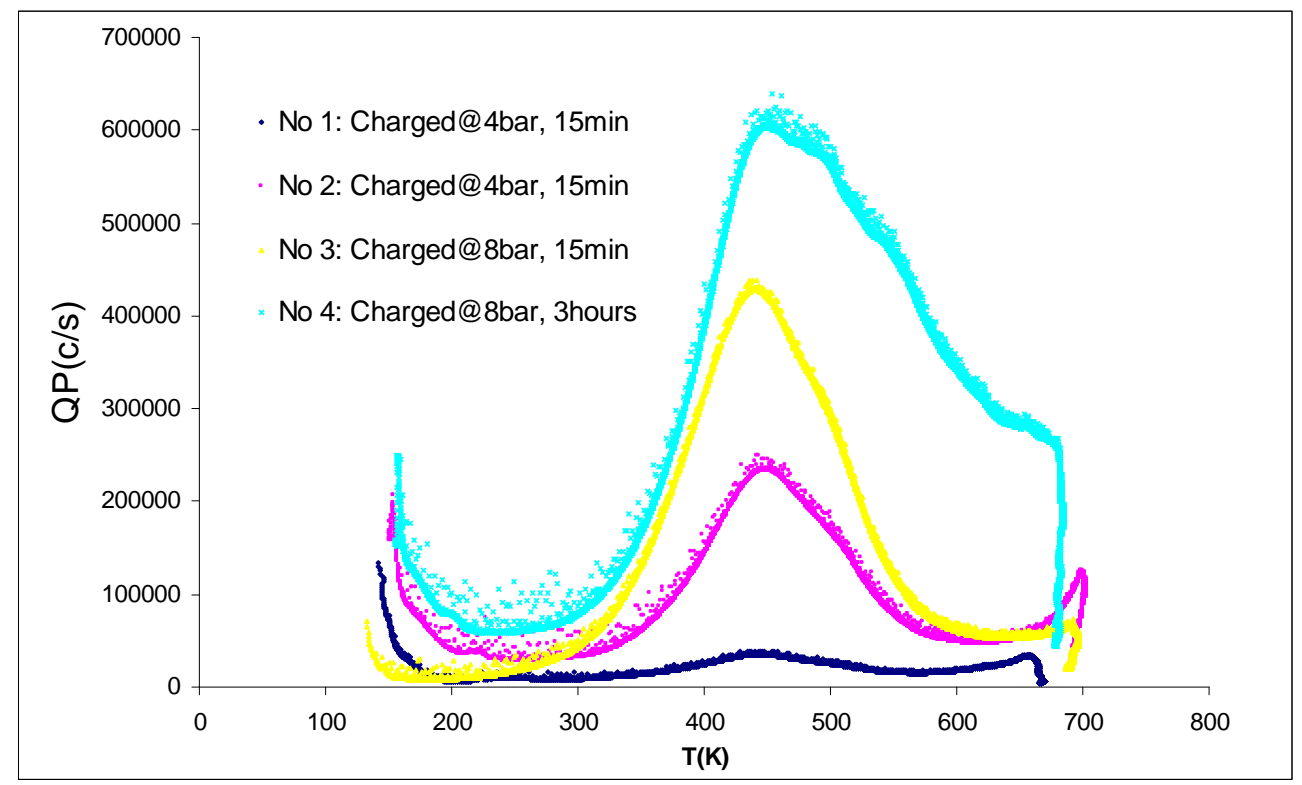

Figure 3.18 Hydrogen desorption measurements of Mg nanoparticles (Tabrizi et al., 2007)

The milled $\mathrm{MgH}_{2} 5$ at.\% $\mathrm{V}$ composite has been reported to desorb the hydrogen at $473 \mathrm{~K}$ $\left(200^{\circ} \mathrm{C}\right)$ under vacuum (Liang et al., 2004). This is close to our results which were obtained without using any catalyst. It should be noted that spark discharge method has the flexibility of inserting the catalyst on the particles by using doped electrodes or making advantage of the mixing properties of the spark (see the next chapter).

\footnotetext{
${ }^{17}$ These measurements were performed in the Fundamental Aspects of Materials and Energy group, RRR, Faculty of Applied Sceiences, TUDelft.
} 


\subsubsection{Pd Nanoparticles}

In comparison with magnesium, palladium is chemically more resistant ${ }^{18}$ and presents high hydrogen solubility over a wide range of pressures and temperatures (Lewis, 1967).

The enthalpy and entropy of formation of $\mathrm{PdH}_{0.6}$ at room temperature are $41.1 \mathrm{~kJ} \mathrm{~mol}^{-1}$ and $97.8 \mathrm{~J} \mathrm{~mol}^{-1} \mathrm{~K}^{-1}$ per mole $\mathrm{H}_{2}$ (Zuttel et al., 2000).

Hydrogen can occupy one octahedral interstitial site $(\mathrm{r}=0.451 \AA)$ and two tetrahedral sites $(\mathrm{r}$ $=0.245 \AA$ ) per atom palladium in the fcc structure of Pd. Here, $r$ denotes the maximum sphere radius to be placed in interstitial space. It may be expected that only interstitial sites with $\mathrm{r}>0.4 \AA$ can be taken by a hydrogen atom ${ }^{19}$ (Zuttel et al., 2000) hence only the octahedral site can host the hydrogen atom in bulk Pd (Figure 3.19), (Kuji et al., 2002). However, it was shown in recent studies that also the tetrahedral positions are occupied to a certain fraction, depending on the conditions (temperature, loading) of the sample (Nanu et al., 2008; Pitt et al., 2003).

It is reported that in nano crystalline $\mathrm{Pd}$, produced by repeated extrusion-compression cycles, the miscibility gap (plateau in P-C isotherm) narrowed with decrease in size (Kuji et al., 2002 ), and the maximum hydrogen concentration in the $\beta$ phase was reduced by almost $50 \%$ (Kuji et al., 2002) indicating that $50 \%$ of available interstitial sites disappeared in nanocrystalline $\mathrm{Pd}^{20}$ or the distribution of interstitial site energy was broadened to higher energy region due to anisotropic strain. One plausible cause explaining that the occupation is reduced may be the lower binding strength of $\mathrm{H}$ at the surface, edges and ridges of particles (Züttel et al., 2004).

In the present work, we produced Pd particles of around $4 \mathrm{~nm}$ in size to test their hydrogen sorption behaviour. The samples were handled in a glove box. The charging and discharging conditions were similar to the magnesium samples.

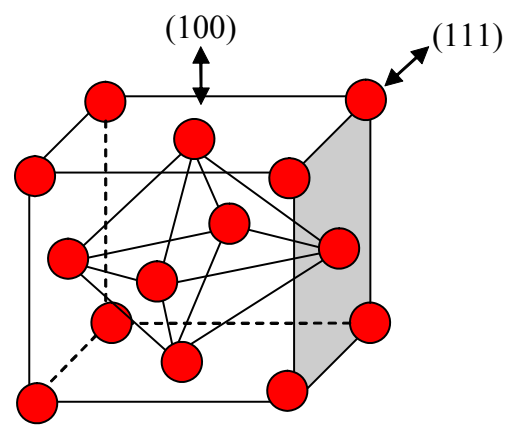

Figure 3.19 Octahedron in fcc Pd (reproduced from Kuji,2002)

Figure 3.20 shows the variation of pressure vs. temperature. The first broad peak at around $200 \mathrm{~K}$ may be attributed to the release of hydrogen from the metal hydride solid solution and the second peak at $250 \mathrm{~K}$ may be due to desorption from the $\alpha$ and $\beta$ coexisting phases (Appendix D).

\footnotetext{
${ }^{18}$ Palladium hydride is sensitive to carbon monoxide impurity.

${ }^{19}$ At low concentrations up to $\mathrm{PdH}_{0.02}$ the palladium lattice expands slightly, from $3.889 \AA$ to $3.895 \AA$. Above this concentration the second phase appears with a lattice constant of $4.025 \AA$. Both phases coexist until a composition of $\mathrm{PdH}_{0.58}$ when the alpha phase disappears.

${ }^{20}$ The reduced hydrogen solubility can also be due to the fact that the surface atoms provide less interstitial sites for hydrogen (Züttel et al., 2001).
} 
It can be seen that the maximum hydrogen release take place at around $250 \mathrm{~K}$. The temperature of $250 \mathrm{~K}$ of the main peak (corresponding to $\alpha-\beta$ phase coexistence) is low: about $70 \mathrm{~K}$ lower than for powder Pd samples (Stern et al., 1984). Figure 3.21 depicts the accumulative hydrogen desorption of our palladium nanoparticles indicating that the maximum weight percentage of the stored hydrogen is quite close to the theoretical maximum value $\left(0.56 \mathrm{wt} \%\right.$ for $\left.\mathrm{Pd} \mathrm{H}_{0.6}\right)$.

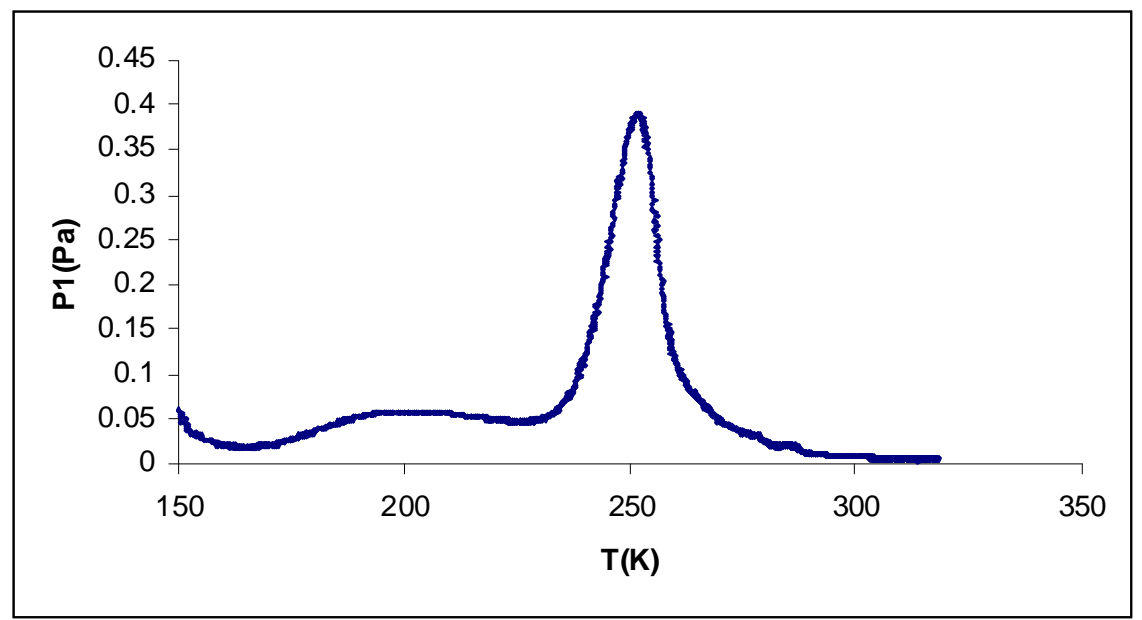

Figure 3.20 Variation of pressure vs. temperature for Pd nanoparticles

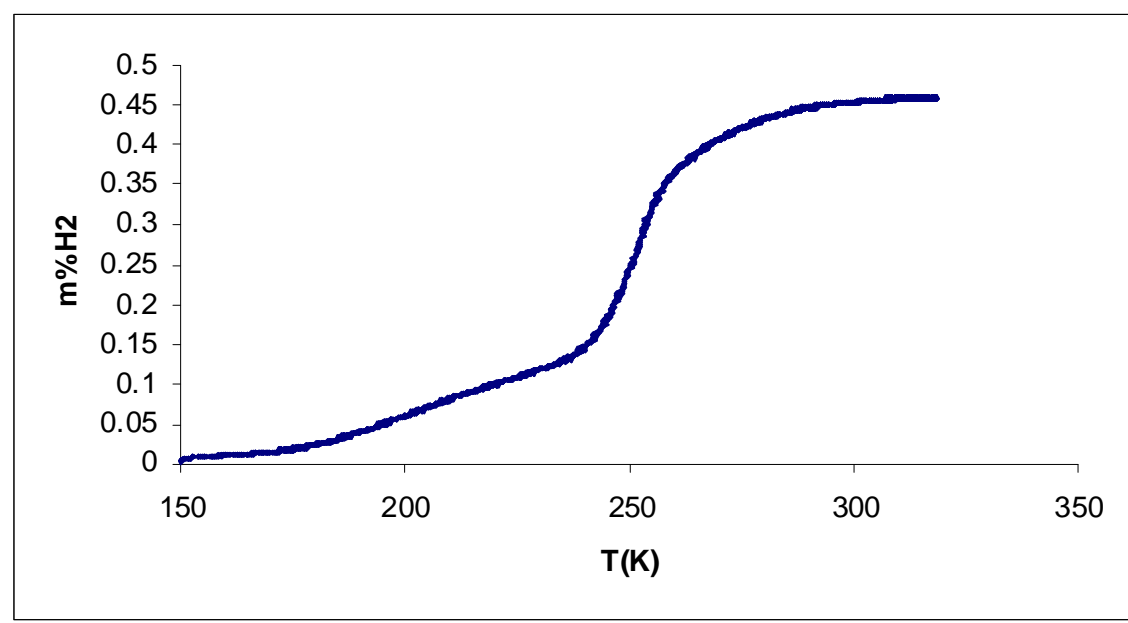

Figure 3.21 Accumulative hydrogen desorption measurement of Pd nanoparticles

\subsection{Conclusion}

In this chapter, various metallic particles were synthesized. Electron microscopy and specific surface area measurements were utilized to characterize them. It was revealed that the particles were mainly nano-sized with an evaporation-condensation formation mechanism. The presence of few large particles among huge number of nanoparticles indicated that parallel to the above-mentioned mechanism, particle formation by ejection of molten metal 
from the electrode surface also occurs. Au particles exhibit pronounced sintering on the substrate, forming continuous random branched structures and indicating high purity of the particle surfaces. Evidently sintering stops or continues very slowly, when the minimum radii of curvature have sufficiently been increased to decrease easy diffusion of surface atoms. In some cases the deviation between TEM and BET diameters for some materials, mainly noble metals was attributed to this inter-particle sintering as well as the presence of large particles. The presence of oxide layers on the particle surfaces evidently hinders sintering. However, oxidation of metallic particles is undesirable for many applications. To achieve unoxidized nanoparticles, a special set-up was utilized which produced such clean particles that they burned out upon sudden exposure to air.

Although the particles produced in this work can potentially have very interesting applications, we studied only two special cases. The antimony/antimony oxide particles which were purposely exposed to air were applied as the anode electrode in a Li-ion battery. Their electrochemical behaviour was studied by Simonin, and it was observed that the texture of particles had a strong effect on the insertion of Li (Simonin et al., 2007). In view of improving hydrogen sorption behaviour by reducing particle sizes, we produced $\mathrm{Mg}$ and $\mathrm{Pd}$ particles of a few nanometers in size and studied their desorption behaviour. For magnesium we observed a clearly reduced desorption temperature $\left(180^{\circ} \mathrm{C}\right)$. The difference between the fairly high observed hydrogen storage capacity of $6.3 \mathrm{wt} . \%$ and the theoretical maximum of 7.6 wt. \% could be due to oxidation. Other samples showed a smaller $\mathrm{H}_{2}$ storage capacity, and this can be attributed to a higher degree of oxidation. Since the spark discharge method offers the possibility of inserting catalyst on the particles (for example by using doped electrodes), further improvement could probably be achieved quite easily. With Pd nanoparticles, we also observed a desorption temperature of about $70 \mathrm{~K}$ lower than for Pd powders, and a hydrogen storage capacity near the value of $0.56 \mathrm{wt} \%$ for bulk Pd.The shape of the thermal desorption spectra is similar to those for Pd powders.

\section{References}

Ashby M. F., a First Report on Sintering Diagrams, Acta Metallurgica, Volume 22, 1974, pp. 275-289

Bryngelsson Hanna, Jonas Eskhult, Kristina Edstrom, Leif Nyholm, Electrodeposition and Electrochemical Characterisation of Thick and Thin Coatings of $\mathrm{Sb}$ and $\mathrm{Sb} / \mathrm{Sb}_{2} \mathrm{O}_{3}$ Particles for Li-Ion Battery Anodes, Electrochimica Acta, Volume 53, 2007, pp. 1062-1073

Castro Facundo J., Gabriel Meyer, a Novel Thermal Desorption Spectroscopy Apparatus, Review of Scientific Instruments, Volume 71, Number 5, 2000, pp. 2131-2133

Cooper D., C. Y. Wu, D. Yasensky, D. Butt, M. Cai, Hydrogen Storage Characteristics of Nickel Nanoparticle Coated Magnesium Prepared by Dry Particle Coating, KONA, Number 23, 2005, pp. 139-151

Dailly A., R. Schneider, D. Billaud, Y. Fort, P. Willmann, New Graphite-Antimony Composites as Anodic Materials for Lithium-Ion Batteries, Electrochemica Acta, Volume 47, 2002, pp. 4207-4212

de Jongh Petra E., Rudy W. P. Wagemans, Tamara M. Eggenhuisen, Bibi S. Dauvillier, Paul B. Radstake, Johannes. D. Meeldijk, John W. Geus, Krijn P. de Jong, The Preparation of Carbon-Supported Magnesium Nanoparticles Using Melt Infilteration, Chemistry of Materials, Volume 19, 2007, pp. 6052-6057

Fernández J. F., C. R. Sanchez, Rate Determining Step in the Absorption and Desorption of Hydrogen by Magnesium, Journal of Alloys and Compounds, Volume 340, 2002, pp. 189-198 
Fernández J. F., C. R. Sanchez, Simultaneous TDS-DSC Measurements in Magnesium Hydride, Journal of Alloys and Compounds, Volume 356-357, 2003, pp. 348-352

Gray Eoin W., Julian R. Pharney, Electrode Erosion by Particle Ejection in Low-Current Arcs, Journal of Applied Physics, Volume 45, Number 2, 1974, pp. 667-671

Hinds William C., Aerosol Technology, Properties, Behaviors,, and Measurements of Airborne Particles, John Wiley\&Sons, 1999

Hirai Makoto, Ashok Kumar, Wavelength Tuning of Surface Plasmon Resonance by Annealing Silver-Copper Nanoparticles, Journal of Applied Physics, Volume 100, 2006, pp. 014309

Karmhag R., Niklasson Gunnar A., M $\backslash$. Nygren, Oxidation Kinetics of Nickel Nanoparticles, Journal of Applied Physics, Volume 89, Number 5, 2001 pp. 3012-3017

Klassen Thomas, Wolfgang Oelerich, Rudiger Bormann, Nanocrystalline Mg-Based Hydrides: Hydrogen Storage for the Zero-Emission Vehicle, Materials Science Forum, Volume 360-362, 2001, pp. 603-608

Kuji Toshiro, Yoshihito Matsumura, Hirishisa Uchida, Tatsuhiko Aizawa, Hydrogen Absorption of Nanocrystalline Palladium, Journal of Alloys and Compounds, Volume 330-332, 2002, pp. 718-722

Kutz Myer, Handbook of Materials Selection, John Wiley\&Sons, 2002

Lafont Ugo, Loic Simonin, Nooshin Salman Tabrizi, Andreas Schmidt-Ott, Erik Kelder, Synthesis of Nanoparticles of $\mathrm{Cu}, \mathrm{Sb}, \mathrm{Sn}, \mathrm{SnSb}$, and $\mathrm{Cu}_{2} \mathrm{Sb}$ by Densification and Atomization Process, Journal of Nanoscience and Nanotechnology, Volume 9, 2009, pp. 2546-2552

Lahiri Debdutta, Bruce Bunker, Bhoopesh Mishra, Bimetallic Pt-Ag and Pd-Ag Nanoparticles, Journal of Applied Physics, Volume 97, 2005, pp. 094304

Lewis F. A., Palladium Hydrogen System, Academic press, 1967

Li Hong, Lihong Shi, Qing Wang, Liquan Chen, Xuejie Huang, Nano-Alloy Anode for Lithium Ion Batteries, Solid State Ionics, Volume 148, No. 3-4, 2002, pp. 247-258

Liang G., Synthesis and Hydrogen Storage Properties of Mg-Based Alloys, Journal of Alloys and Compounds, Volume 370, 2004, pp. 123-128

Nalwa Hari Singh, Nanoclusters and Nanocrystals, American Scientific Publishers, 2003

Nanu D. E., W. J. Legerstee, S. W. H. Eijt, W. G. Haije, J. F. Vente, M. G. Tucker, A. J. Böttger, Insights into the Relation between Crystal Structure and Deuterium Desorption Characteristics of Pd-Au-D Alloys, Acta Materialia, Volume 56, 2008, pp. 6132-6140

Niklasson Gunnar A., Richard Karmhag, Oxidation Kinetics of Metallic Nanoparticles, Surface Science, Volume 532-535, 2003, pp. 324-327

Oelerich Wolfgang, Thomas Klassen, Rudiger Bormann, Hydrogen Sorption of Nanocrystalline Mg at Reduced Temperatures by Metal-Oxide Catalysts, Advanced Engineering Materials, Volume 3, Number 7, 2001, pp. 487490

Petr R. A., T. R. Burkes, Acoustic Phenomena in Erosion of Spark-Gap Electrodes, Appl. Phys. Lett., Volume 36, Number 7, 1980, pp. 536-537

Pitt M. P., E. Mac A. Gray, Europhys. Lett., Volume 64, 2003, pp. 344-350.3

Poole Jr. Charles P., Frank J. Owens Frank J., Introduction to Nanotechnology, WILEY, 2003

Rao C. N. R., G. U. Kulkarni, A. Govindaraj, B. C. Satishkumar, and P. John Thomas, Metal Nanoparticles, Nanowires, and Carbon Nanotubes, Pure Applied Chemistry, Volume 72, Number 1-2, 2000, pp. 21-33 
Rouquerol Francoise, Jean Rouquerol, Kenneth Sing, Adsorption by Powders and Porous Solids: Principles, Methodology and Applications, San Diego Academic Press, 1999

Sanchez-Lopez J. C., C. Caballero, A. Fernandez, Characterization of Passivated Aluminium Nanopowders : An XPS and TEM/EELS Study, Journal of European Ceramics Society, Volume 18, 1998, pp. 1195-1200

Simonin L., U. Lafont, N. S. Tabrizi, A. Schmidt-Ott, E. M. Kelder, Sb/O Nano-Composites Produced via Spark Discharge Generation for Li-Ion Battery Anodes, Journal of Power Sources, Volume 174, Number 2, 2007 , pp. 805-809

Soon Kwon Y., A. Gromov, A. P. IIyin, A. Ditts, J. Soon Kim, S. Ha Park, M. Hee Hong, Features of Passivation, Oxidation and Combustion of Tungsten Nanopowders by Air, International Journal of Refractory Metals \& Hard Materials, Volume 22, 2004, pp. 235-241

Stern A., A. Resnick, D. Shaltiel, Thermal Desorption Spectra of the $\mathrm{PdH}_{\mathrm{x}}$ System in a Powder Form, Journal of Phys. F: Met. Phys., Volume 14, 1984, pp. 1625-1639

Sugimoto Tadao, Fine Particles Synthesis, Characterization, and Mechanisms of Growth, Marcel Dekker, 2000

Tabrizi N. S., W.J. Legerstee, S. Eijt, A. Schmidt-Ott, Hydrogen Storage Behaviour of Metallic Nanoparticles Generated by Spark Discharge, European Aerosol Conference 2007, Salzburg, Abstract T09A026

Wagemans Rudy W. P., Joop H. van Lenthe, Petra E. de Jongh, A. Jos van Dillen, Krijn P. de Jong, Hydrogen Storage in Magnesium Cluster: Quantum Chemical Study, J. Am. Chem. Soc., Volume 127, 2005, pp. 1667516680

Wu M. K., R. S. Windeler, C. K. R. Steiner, T. Börs, S. K. Friedlander, Controlled Synthesis of Nanosized Particles by Aerosol Processes, Aerosol Science and Technology, Volume 19, 1993, pp. 527-548

Xu C. H., S. Q. Shi, C. Surya, C. H. Woo, Synthesis of Antimony Oxide Nano-Particles by Vapor Transport and Condensation, journal of Materials Science, Volume 42, Number 23, 2007, pp. 9855-9858

Yavari A. R., J. F. R. de Castro, G. Vaughan, G. Heunen, Structural Evolution and Metastable Phase Detection in $\mathrm{MgH}_{2}-5 \% \mathrm{NbH}$ Nanocomposite during in-situ H-Desorption in a Synchrotron Beam, Journal of Alloys and Compounds, Volume 353, 2003, pp. 246-251

Zaluska A., L. Zaluski, J. O. Strom-Olsen, Nanocrystalline Magnesium for Hydrogen Storage, Journal of Alloys and Compounds, Volume 288, 1999, pp. 217-225

Zaluska A., Structure, Catalysis and Atomic Reactions on the Nano-Scale: a Systematic Approach to Metal Hydrides for Hydrogen Storage, Appl. Phys. A, Volume 72, 2001, pp. 157-165

Zeng P., S. Zajac, P. C. Clapp, J. A. Rifkin, Nanoparticle Sintering Simulations, Materials Science and Engineering A, Volume 252, 1998, pp. 301-306

Züttel A., Ch. Nutzenadel, G. Schmid, Ch. Emmenegger, P. Sudan, L. Schlapbach, Thermodynamic Aspects of the Interaction of Hydrogen with Pd Clusters, Applied Surface Science, Volume 162-163, 2000, pp. 571-575

Züttel A., P. Wenger, P. Sudan, P. Mauron, S. Orimo, Hydrogen Density in Nanostructured Carbon, Metals and Complex Materials, Mater. Sci. Eng. B, Volume 108, 2004, pp. 9-18 


\section{Chapter 4}

\section{Generation of Bimetallic Nanoparticles from Miscible Metals}

\subsection{Introduction}

Bimetallic nanoparticles are of fundamental and technological interest due to their size, composition and structure dependent properties. The relative concentrations of the constituents of bimetallic nanoparticles can be employed to optimize magnetic properties, to control the surface plasmon band and to tailor catalyst properties (Regen et al., 2006). Bimetallic nanocatalysts may offer a synergistic performance leading to enhancement in activity and selectivity (Hirakawa et al., 2003). Thus, there is a great interest in developing new methods for synthesis of bimetallic nanoparticles. Therefore, the main objective of this chapter is to investigate the feasibility of mixed nanoparticle generation by spark discharge. This may open a new field of applications for this technique.

Bimetallic nanoparticles can be synthesized by physical or chemical routes. Physical procedures usually involve evaporation-condensation process whereas chemical methods involve simultaneous or successive reduction of two metal ions in presence of a suitable stabilizer in the liquid phase. One disadvantage of the latter route is the introduction of impurities associated with the chemical solvents in contrast to the gas-phase synthesis which offers high purity. In particular, the spark discharge method offers great flexibility concerning the product composition by applying suitable electrodes.

The present study is restricted to the metals that fulfil the conditions of forming a substitutional solid solution in the bulk phase (Hume-Rothery et al., 1954) and are miscible in macroscopic phases ${ }^{1}$.

Alloyed electrodes of Cr-Co were applied to produce mixed nanoparticles. The evaporation rate of $\mathrm{Cr}$ is much higher than the evaporation rate of Co (Kubaschewski, 1979; Powell et al., 1997). So we deliberately chose a "tough case" to test the fast quenching SDG ${ }^{2}$ method in view of obtaining a mixed phase. In addition, we studied mixing by the discharge process, using compositionally different electrodes on the examples of Au-Pd and Ag-Pd. In the case of noble metals, $\mathrm{Au}$ and $\mathrm{Pd}$ have a rather similar evaporation rate while $\mathrm{Ag}$ and $\mathrm{Pd}$ have a very different one (Kubaschewski, 1979; Powell et al., 1997). It is of interest whether the mixing behaviour is similar.

The examples we chose have applications in various fields. For instance thin films of $\mathrm{Co}-\mathrm{Cr}$ based magnetic alloys have applications as longitudinal high-density recording media (Kahng et al., 1999). In addition, chromium-cobalt alloys are used in surgical implants, and there is concern about the long-term internal exposure to the nanoparticulate wear debris generated

\footnotetext{
${ }^{1}$ For substitutional solid solutions, according to the Hume-Rothery rules, solid solubility requires a small lattice constant mismatch $(\leq 15 \%)$ and identical crystal structures of the two elements. The solute and solvent should typically have similar electronegativities and maximum solubility occurs when the valence of solute and solvent are the same. Metals with lower valence will tend to dissolve metals with higher valence (Considine, 2002).

${ }^{2}$ Spark Discharge Generation (SDG)
} 
from these alloys (Papageorgiou et al., 2007). Particles of the composition of the implants are required for biocompatibility and toxicology studies.

Noble metals (Ag, Au, and $\mathrm{Pd}$ ) are generally considered as useful alloying metals due to their low reactivity. Besides, noble metals have numerous applications (Devarajan et al., 2005; Yang et al., 2006). For instance Au-Pd alloys are reported as the most effective catalysts yet identified for remediation of trichloroethene (TCE), one of the most troublesome groundwater pollutants (Nutt et al., 2006). Ag-Pd alloy particles are commonly applied in electronic devices for their specific electrical properties (Keskinen et al., 2004; Silvert et al., 1996)) for example in catalyzing electroless copper deposition which is an important process in circuitry formation for $\mathrm{PCB}^{3}$ (Yang et al., 2004). Moreover, noble metal nanoparticles are known to possess high optical nonlinearities and ultra-fast optical responses, and they may be utilized in developing nonlinear optical devices (Kim et al., 2003).

\subsection{Experimental Method}

In the spark discharge process, nanoparticles are generated by an evaporation-condensation mechanism. If different materials co-evaporate from the electrodes, co-condensation should lead to formation of mixed particles (Appendix C).

The experimental setup is schematized in Figure 2.5. It consists of the synthesis, collection, and on-line particle size measurement units. The particles are generated under an inert atmosphere (Ar, 99.999\%), and collected on a membrane filter fixed in an air-tight stainless steel holder mounted at the outlet of the generator. The carrier gas flow rate is variable from 0.8 to $5 \mathrm{lpm}$.

High resolution electron microscopy was performed on a Philips CM30-UT-FEG and a FEI Tecnai-200FEG. EDS data were acquired on a FEI Tecnai-200FEG using the STEM mode with a spot size of $\sim 0.2 \mathrm{~nm}$, which guaranteed that the measured spectra were only coming from each single particle. The spectra acquisition, drift correction and data analysis were all processed using the software TIA (Tecnai Imaging \& Analysis). The TEM grid was Ni 200 mesh coated with carbon film. The X-ray diffraction (XRD) measurements were performed on a Bruker -AXS D5005 diffractometer, equipped with a Huber CuKalpha-1 Ge monochromator in the incident beam and a Braun Position Sensitive Detector PSD-50M in the diffracted beam. For the Co-Cr sample, a Bruker-AXS theta-theta diffractometer with a $\mathrm{CuKalpha}$ graphite monochromator in the diffracted beam was used to suppress fluorescence from the sample. The average compositions of samples were measured in PerkinElmer Optima 5300 using the ICP-OES technique. $\mathrm{N}_{2}$ adsorption isotherms were measured on a

Quantachrome Autosorb-6B for the determination of the specific surface areas of the particles.

After sampling for electron microscopy, the sample holder was sealed by valves on both sides to protect the Co-Cr particles from air exposure.

This holder was brought to a glove box, where the TEM grid was transferred to a vacuum sampler. The latter was brought into the microscope so that the particles could be analyzed without having been exposed to air.

\footnotetext{
${ }^{3}$ Printed Circuit Board (PCB)
} 


\subsection{Results and Discussion}

\subsubsection{Study of Various Systems}

\subsubsection{Cr-Co}

These particles were produced by using two electrodes consisting of a Cr-Co alloy. Electron micrographs of the particles and the corresponding EDS spectrum are shown in Figure 4.1 and Figure 4.2. The primary particles were spheres around $4 \mathrm{~nm}$ in diameter. As seen in Figure 4.1, few large round particles in the range of several tens of nanometres were observed in addition to the nanoparticles. As discussed in the previous chapter, we assume that these particles are formed by ejection of liquid material from the electrodes.

In addition to $\mathrm{Cr}$ and $\mathrm{Co}$, EDS analysis showed also $\mathrm{O}, \mathrm{Si}$, and Mo. This is consistent with the composition of the electrodes, which contained up to $1 \mathrm{wt} \% \mathrm{Si}$ and $5 \mathrm{wt} \%$ Mo. The oxygento-metal atomic ratio varied in different regions of the sample. When the synthesis was performed under $\mathrm{Ar}+5 \% \mathrm{H}_{2}$, EDS analysis showed a smaller oxygen content. Precise quantification of oxygen failed due to overlapping of the peak with the metallic species.
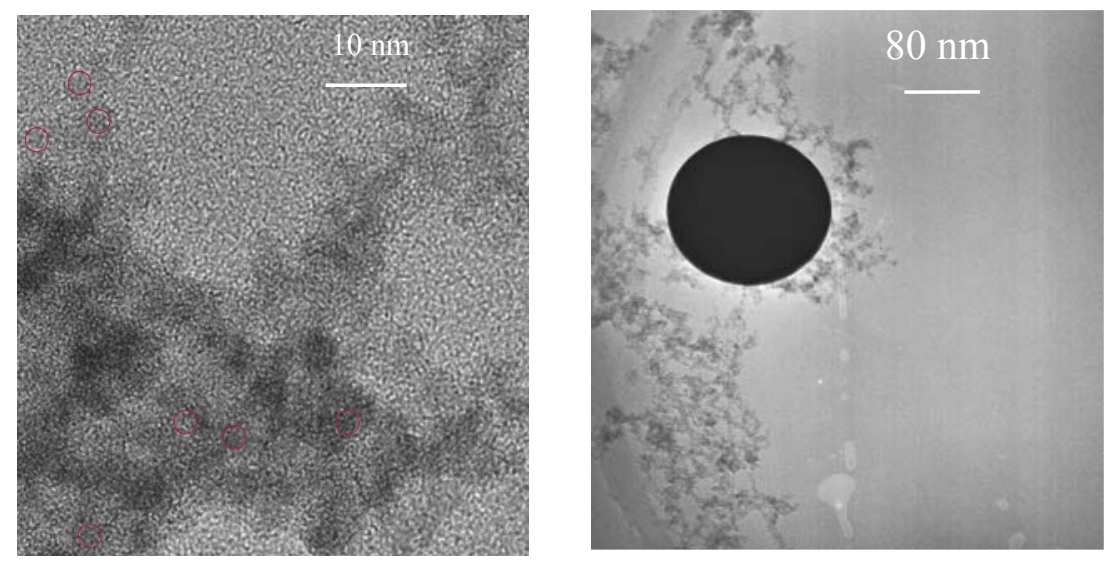

Figure 4.1 TEM images of Cr-Co nanoparticles $\left(\mathrm{C}=20 \mathrm{nF}, d=1 \mathrm{~mm}, \mathrm{f}=150 \mathrm{~Hz}, \mathrm{Q}=1 \mathrm{lpm} \mathrm{Ar}+5 \% \mathrm{H}_{2}\right)$

The oxygen seen in the EDS spectrum is due to $\mathrm{O}_{2}$ adsorbates on the TEM grids and/or to a slight surface oxidation due to $\mathrm{O}_{2}$ impurities in the inert gas. The latter is reduced by the presence of hydrogen. The deposit looked completely black in the glove box, indicating metallic particles. When exposed to air the colour turned green, indicating formation of oxide phase(s) as $\mathrm{Cr}_{2} \mathrm{O}_{3}, \mathrm{CoO}$, and $\mathrm{CoCr}_{2} \mathrm{O}_{4}$ (Fernandez, 2002).

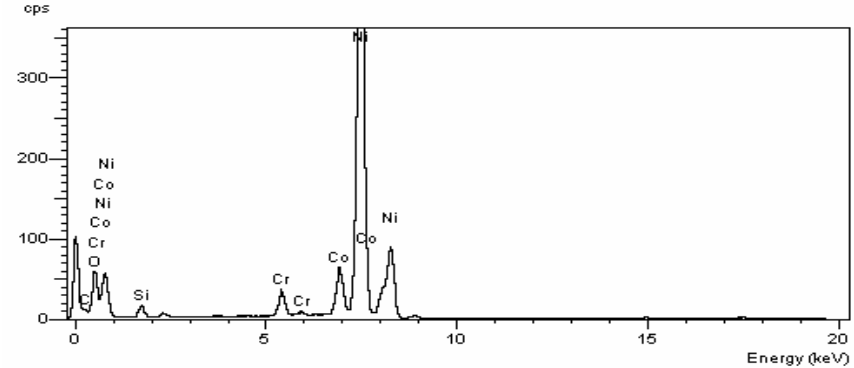

Figure 4.2 EDS spectrum of Cr-Co nanoparticles 
Table 4.1 indicates the ratio of $\mathrm{Cr}$ to $\mathrm{Co}$ in the nanoparticulate powder, as measured by the ICP method. It is consistent with the composition of the electrodes. This is an important result, which shows that $\mathrm{Co}$ and $\mathrm{Cr}$ behave similarly in the rapid evaporation-condensation process despite the differences in their relevant physical constants. Temporary local melting apparently does not lead to any significant segregation.

Table 4.1 Composition of Cr-Co in the electrodes and in the sample measured by ICP

\begin{tabular}{|l|l|l|}
\hline Electrodes & $\mathrm{Cr} /$ Co weight ratio in the electrode & $\mathrm{Cr} /$ Co weight ratio in the sample \\
\hline Cr-Co alloy & $0.37-0.51$ & 0.37 \\
\hline
\end{tabular}

Figure 4.3 shows the $\mathrm{X}$ - ray diffraction patterns of the Cr-Co sample as measured with the background subtracted. The sample had been exposed to air before the analysis. XRD patterns show some sharp peaks superimposed on broad peaks. These sharp peaks confirm the presence of big particles as seen in the TEM micrograph (Figure 4.1) and the broad peaks are due to nanoparticles. The area under the sharp peaks is much smaller than the area under the broad peaks. This indicates that the sample is mainly composed of the nanocrystalline phase. There are peaks that match the diffraction data of the chromium oxide and cobalt oxide phases which have evidently been formed by exposure to air. As we neither see any mixed oxide phase nor any pure metallic phase, we infer that oxidation may lead to separation of the $\mathrm{Cr}$ and $\mathrm{Co}$.

There is also a Co-like pattern with a lattice parameter increased by a factor of 1.0083 which we interpret as a mixed $\mathrm{Co}-\mathrm{Cr}$ crystal phase. Chromium has a body-centred cubic (bcc) structure and cobalt is an allotropic metal with hexagonal close-packed (hcp) and facecentered cubic (fcc) crystal structures. Although the hexagonal-closed-packed cobalt structure is thermodynamically favoured, formation of the face-centered-cubic (fcc) cobalt nanoparticles by flame synthesis has previously been reported (Grass et al, 2006).

In substitutional solid solutions, where the atoms form a common lattice, the constituents usually possess similar atomic radii. The formation of a substitutional solid solution is generally accompanied by an expansion or contraction of the lattice parameters of the solvent (Hume-Rothery et al., 1954).

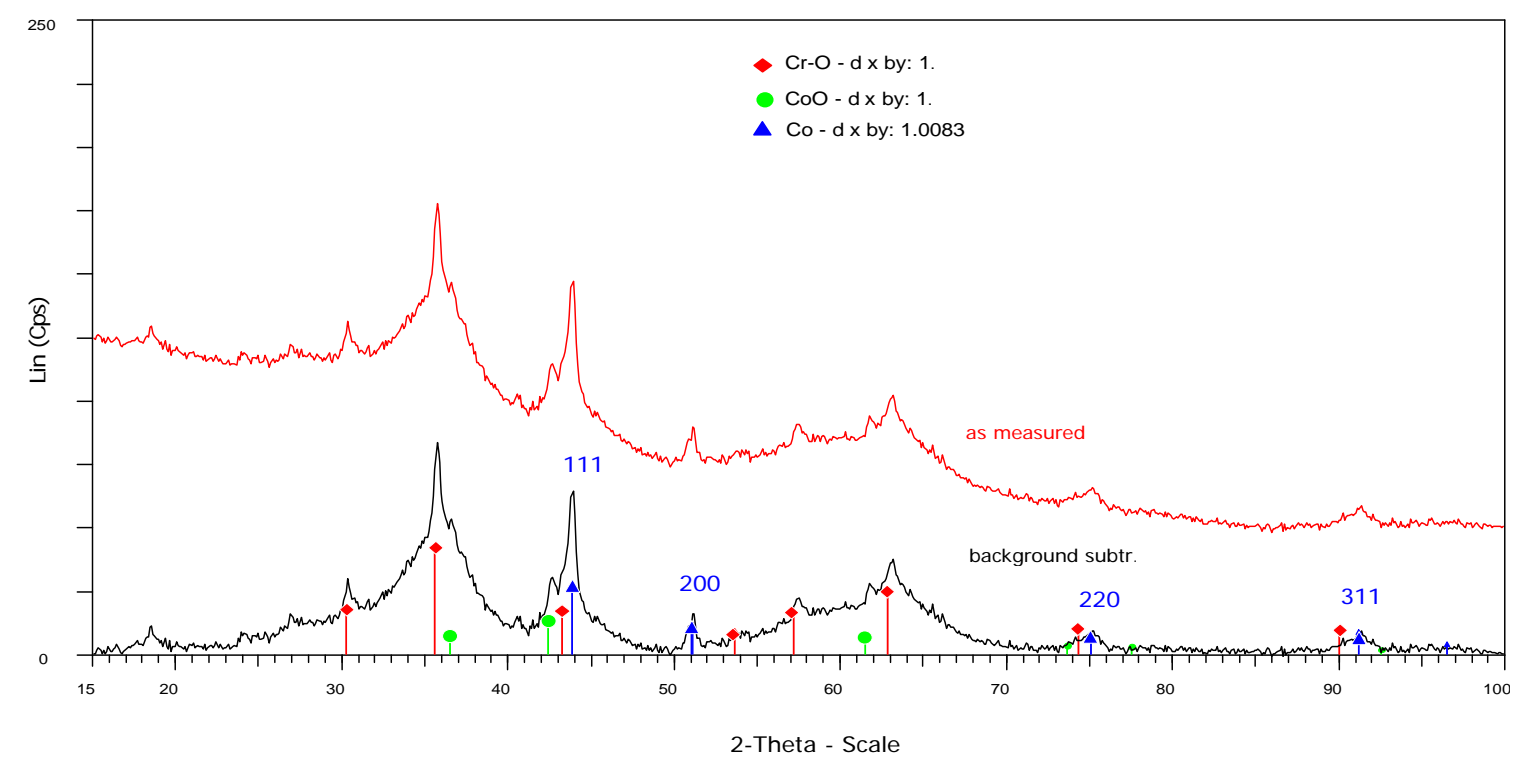

Figure 4.3 XRD patterns of the Cr-Co sample 
According to Co-Cr lattice parameters vs. compositions for fcc $(\alpha-\mathrm{Co})$ solid solutions (Predel et al, 1998), the lattice constant obtained from the X-ray diffraction pattern fitted to about 40 at $\% \mathrm{Cr}$, corresponding to a $\mathrm{Cr} / \mathrm{Co}$ weight ratio of around 0.58 somewhat larger than ICP measurement, which may be due to the formation of the oxide phases.

Table 4.2 shows the specific surface area of the particles, measured by the BET method (Rouquerol et al., 1999) and the corresponding BET diameter which was calculated for spherical shape (shape factor 6) with bulk density between the pure cobalt and pure chromium. In spite of the presence of some large particles (Figure 4.1), the average particle diameter observed by electron microscopy agrees with the calculated BET diameter. This indicates that large particles are rare in the sample, in agreement with the small contribution of sharp peaks mentioned above to the areas under the peaks. The particles partially oxidize before the BET measurement, but it is easily shown that this should only have a small influence on the BET diameter if the density of the metals is used in the calculation.

Table 4.2 Specific surface area of Cr-Co nanoparticles

\begin{tabular}{|l|l|l|l|}
\hline Electrode & BET surface area $\left(\mathrm{m}^{2} / \mathrm{g}\right)$ & Calculated BET diameter $(\mathrm{nm})$ & TEM average diameter $(\mathrm{nm})$ \\
\hline Cr-Co & 185 & $4-5$ & 4 \\
\hline
\end{tabular}

The particle size distribution as measured by the mobility analyser (Figure 4.4) showed a modal diameter of around $7 \mathrm{~nm}$ and a geometric standard deviation of 1.41. The discrepancy with the primary particle size on the TEM micrographs (Figure 4.1) indicates that the particles agglomerate during the time of a few seconds between production and mobility measurement. Using coagulation theory (Hinds, 1999), the initial primary particle concentration close to the spark is estimated to be between $10^{9}$ and $10^{10} \mathrm{~cm}^{-3}$.

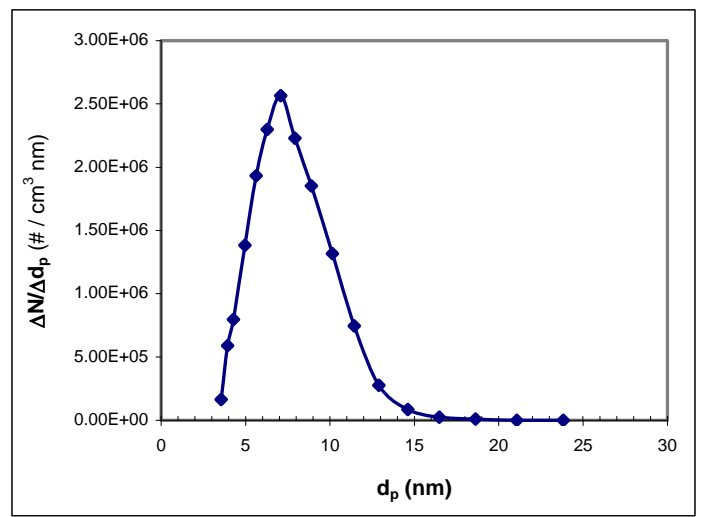

Figure 4.4 Size distribution of Cr-Co nanoparticles $(C=5 \mathrm{nF}, d=1 \mathrm{~mm}, f=10 \mathrm{~Hz}, Q=5 \mathrm{lpm} \mathrm{Ar})$

\subsubsection{Au-Pd}

$\mathrm{Au}-\mathrm{Pd}$ particles were produced by using a gold electrode and a palladium electrode. The vapours produced at the electrode surfaces are expected to mix at least partially. This mixing is driven by rapid diffusion in plasma with a temperature up to several thousands $\mathrm{K}$ as well as by electrical forces acting on the ionized species in the plasma which include the metal vapours. In this context it is important that in the discharge process the voltage across the electrodes performs a damped oscillation, which means that it reverses polarity. The capacitive, inductive and resistive elements in the circuit have been chosen to guarantee this behaviour (Tabrizi et al., 2009). Temporary voltage reversal is also important to achieve ablation of both electrodes. 
TEM micrographs in Figure 4.5 depict the particles at two different magnifications. The primary particles, around $6 \mathrm{~nm}$ in diameter, form a chain-like network and it is clearly seen that they are sintered at the contact areas. It was already pointed out (chapter two) that pure gold nanoparticles produced by the spark exhibit cold sintering. It can be due to purity of the surfaces of the noble metallic particles and absence of any diffusion barrier such as an oxide layer. The rapid sintering of ultra-clean nanoparticles is in agreement with molecular dynamic (MD) simulation results (Koch, 2007). As the network of sintered particles looks continuous, while the DMA spectrum indicates units of a few $\mathrm{nm}$, sintering must have taken place on the substrate and at room temperature. Sintering was not seen in the case of Co-Cr particles, and it can be attributed to chemisorption of gas impurity molecules at the particle surface, probably oxygen (see above).

The SEM images of Au-Pd particles showed that primary particles self-organized in corallike structures, growing in all directions as well as in long threads with lengths in the millimetre range. While fractal-like growth, forming these structures, is a common phenomenon and simply explainable by random diffusion limited aggregation, the formation of long linear threads is astonishing. It could be due to whisker or nanotube growth from the electrode surface, which may be field induced. Bare whiskers are not seen anywhere, but the abundance of nanoparticles should lead to coverage by these. Field-induced formation of linear nanoparticle chains is another possible explanation.

High resolution electron microscopy revealed that the particles were crystalline. This is important, because crystallinity is desired for most applications.
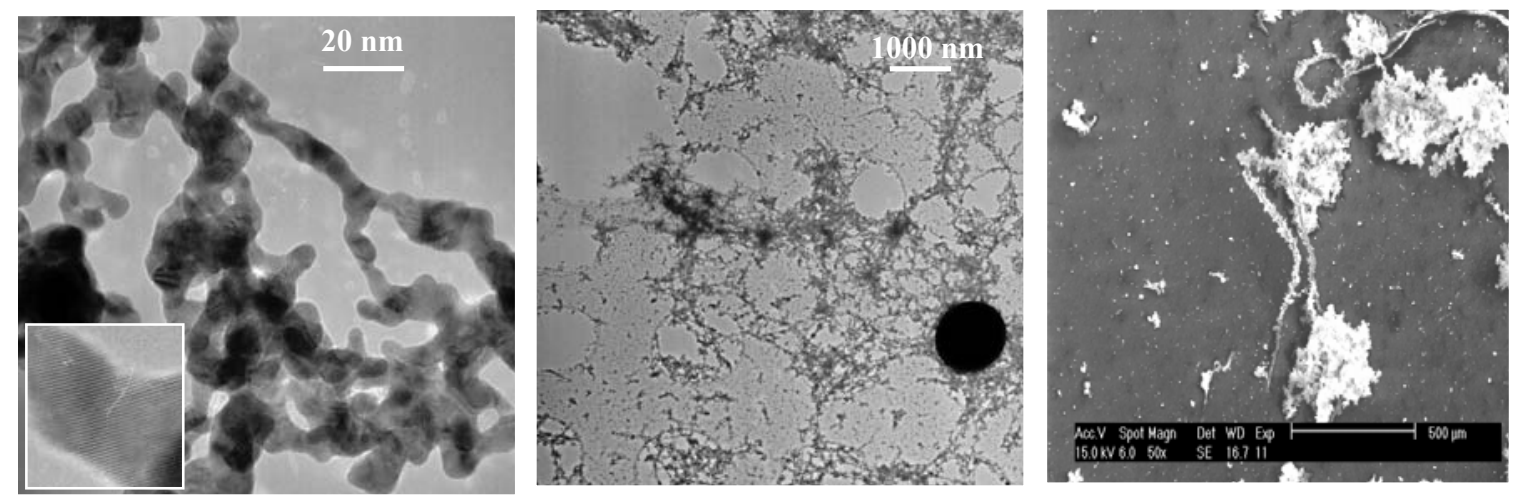

Figure 4.5 TEM and SEM (scale bar $500 \mu \mathrm{m}$ ) images of Au-Pd nanoparticles $(C=20 \mathrm{nF}, d=1 \mathrm{~mm}, f=150 \mathrm{~Hz}, Q=0.8 \mathrm{lpm} \mathrm{Ar}$ )

The average compositions of the particles measured by the ICP method are listed in Table 4.3. It can be concluded that for a specific material the negative electrode is eroded more strongly than the positive one. This is due to the fact that the majority of the charge carriers in the plasma are positive ions and electrons. The ions have a much higher mass and thus dissipate more kinetic energy in colliding with the attracting electrode than the electrons, despite the higher mobility of the latter. The fact that we do see substantial erosion of the positive electrode is due to temporary voltage reversal during the discharge process, as mentioned above.

Table 4.3 Average compositions of Pd-Au samples measured by ICP [Au (+)Pd (-) means that the anode was gold and the cathode was palladium]

\begin{tabular}{|l|l|}
\hline Electrodes & $\mathrm{Pd} / \mathrm{Au}$ weight ratio in the sample \\
\hline $\mathrm{Au}(+) \mathrm{Pd}(-)$ & 0.58 \\
\hline $\mathrm{Au}(-) \mathrm{Pd}(+)$ & 0.34 \\
\hline
\end{tabular}


EDS analysis allowed determination of the composition of individual particles. Particles in the size range of 5-10 $\mathrm{nm}$ were analyzed. Table 4.4 lists the $\mathrm{Pd} / \mathrm{Au}$ weight ratio in six particles, indicating that the particles have a distribution of compositions. This is not surprising and likely due to an uneven distribution of materials in the space between the electrodes.

Table 4.4 EDS compositional analysis for six single nanoparticles [Au(-)Pd(+)]

\begin{tabular}{|l|l|l|l|l|l|l|l|}
\hline \multicolumn{5}{|c|}{$\mathrm{Pd} /$ Au weight ratio } & Average & Std \\
\hline 0.395095 & 0.120512 & 0.072145 & 0.170565 & 0.281923 & 0.497013 & 0.256209 & 0.165871 \\
\hline
\end{tabular}

To investigate the compositional homogeneity in one single particle, EDS mapping was carried out under the scanning transmission electron microscopy (STEM) mode on single particles. A typical particle highlighted by the solid square frame is shown in Figure 4.6.a. This particle is around $5 \mathrm{~nm}$ in diameter and isolated from others. During compositional mapping, the sample drift was corrected by comparing with a reference image, highlighted by the dashed square frame in Figure 4.6.a. The L-edge spectra are used as relative measures of the $\mathrm{Au}$ and $\mathrm{Pd}$ mass encountered in each pixel. The information is represented on a $7 \times 7$ pixel grid covering the particle with each pixel about $0.7 \mathrm{~nm}$ wide. The mappings for $\mathrm{Au}$ and $\mathrm{Pd}$ are shown in Figure 4.6.b and c, respectively. The patterns look quite similar, which means that the two elements are quite mixed within the precision of the method. In particular, the outer boundaries of the particle are practically identical for Pd and for Au. This means that there is no surface segregation, which could generally be driven by surface energy, melting point, affinity of gases, heat of sublimation, and strain energy (Wang et al., 2006). The corresponding effects lead to core-shell structures as this has been reported by Zhang et al., 2005, Okitsu et al., 2000 and Devarajan et al., 2005. The thermodynamic driving force may lead the component with lower surface energy to segregate to the surface (Xiao et al., 2006). The absence of any visible segregation in our particles may be due to the fast quenching process following the spark.

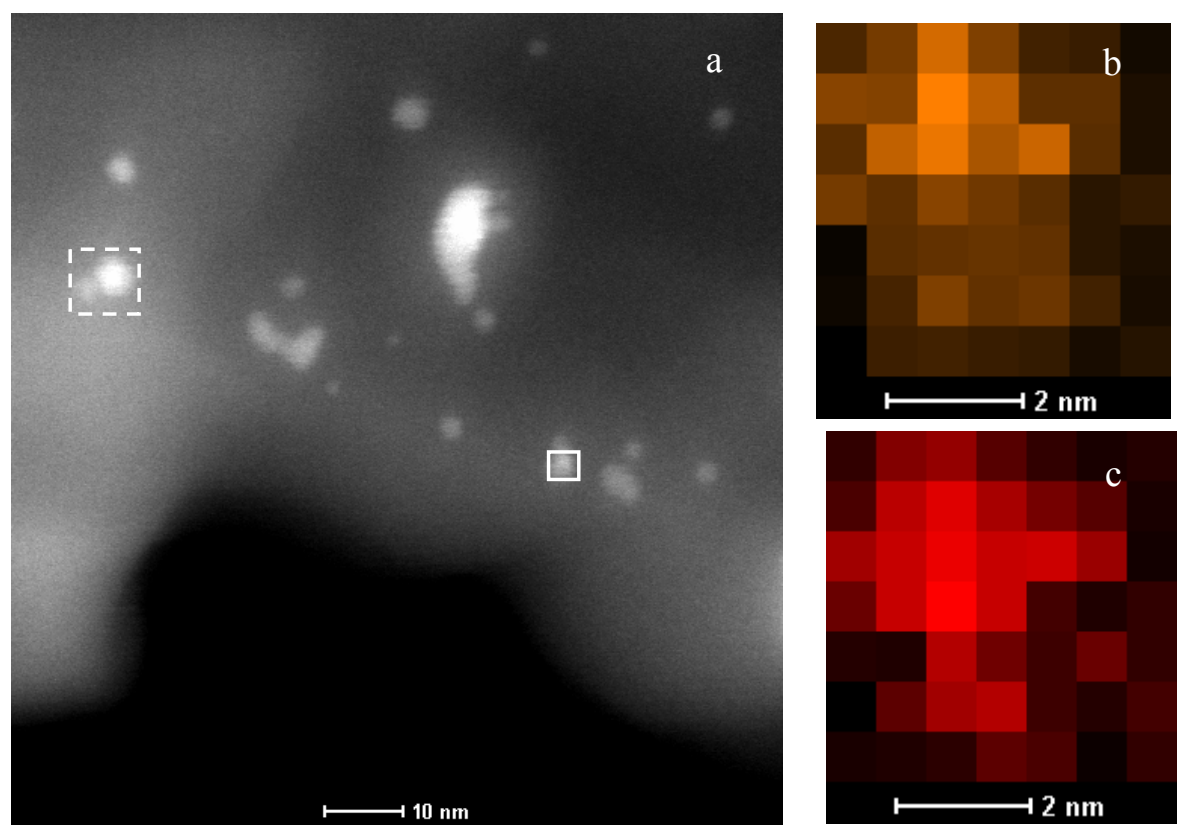

Figure 4.6 Typical STEM maps of a Au-Pd particle (a), AuL (b), PdL (c) 


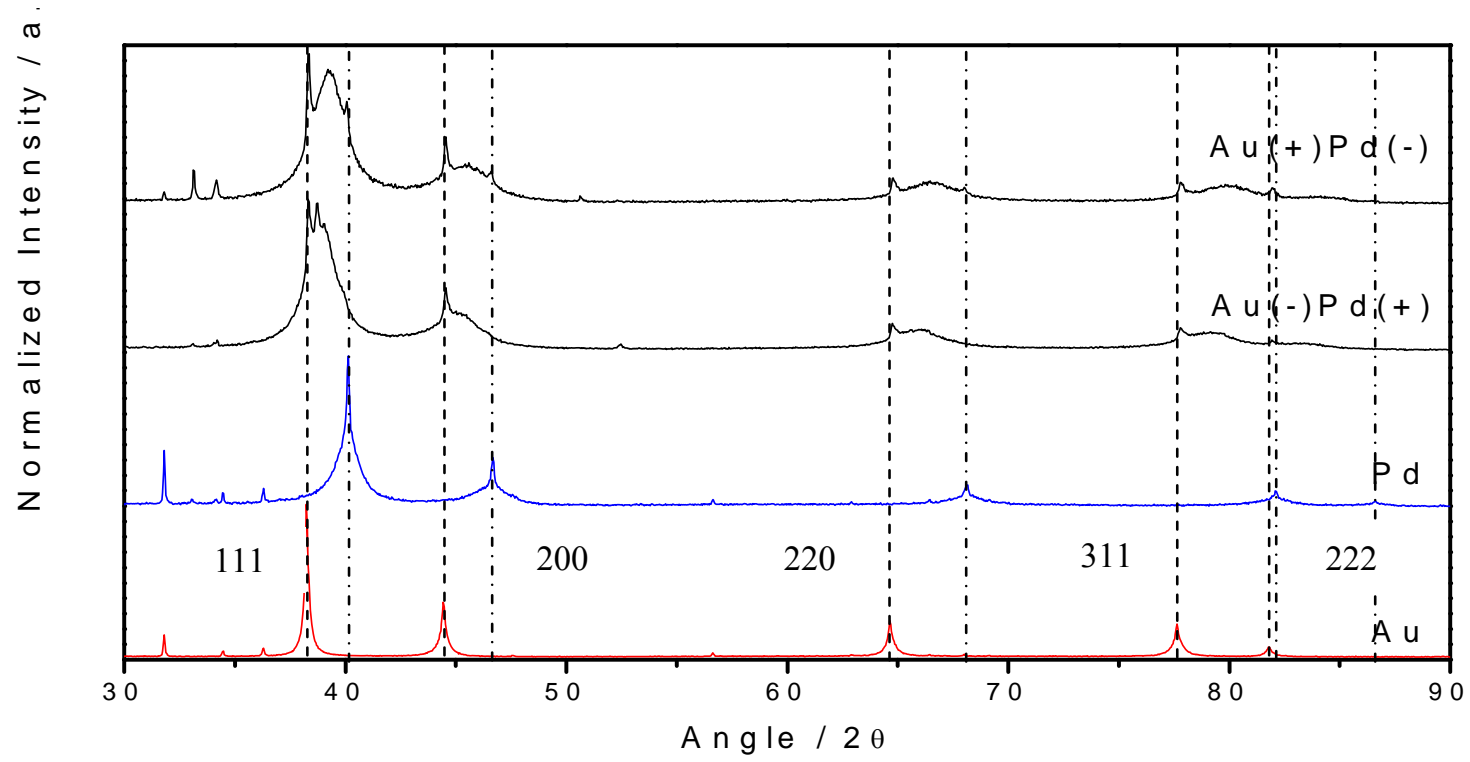

Figure 4.7 XRD patterns of the $A u, P d,[A u(+) P d(-)]$, and $[A u(-) P d(+)]$ nanoparticles

X-ray diffraction patterns were recorded to obtain information about the formation of mixed phases. According to the phase diagram for $\mathrm{Au}-\mathrm{Pd}$, these metals form solid solutions of arbitrary compositions at low temperatures (Liu et al., 2005). Enthalpies of formation of Au$\mathrm{Pd}$ solid solutions (between 423 and $573 \mathrm{~K}$ ) are in the range of -7.1 and $-7.9 \mathrm{~kJ} / \mathrm{g}$-atom (Yang et al., 2004) for the compositions listed in Table 4.3. These large negative values should lead to rapid alloy formation (Menon et al., 2001). The XRD patterns of $\mathrm{Au}, \mathrm{Pd}$ and Au-Pd particles are shown in Figure 4.7.The patterns of the pure phases were produced by using the same material for both electrodes. For the mixed case, the diffraction curve for each polarity is shown. The XRD patterns for the pure metals show the characteristic peaks for Pd and Au. The presence of large particles, and cold sintering in the Au particles resulted in narrow Bragg reflections. The patterns for the mixed cases also show the pure metal peaks. Between them there are broad peaks, which are attributed to the mixed phase.

Gold and palladium both have fcc (face-centered cubic) structures and their lattice constants are close together around 0.40782 and $0.38907 \mathrm{~nm}$ respectively. For metals with the same crystal structures and atomic sizes, Vegard's law predicts a simple linear relationship between alloy compositions and lattice spacing (Barret et al., 1996). Taking the modes of the mixed phase peaks, this law predicts $\mathrm{Pd} / \mathrm{Au}$ weight ratio of $44 \%$ for the $\mathrm{Au}(+) \mathrm{Pd}(-)$ sample and $36 \%$ for the $\mathrm{Au}(-) \mathrm{Pd}(+)$ sample. This is in qualitative agreement with Table 4.3 in the sense that the sample with more $\mathrm{Pd}$ content is produced from $\mathrm{Au}(+) \mathrm{Pd}(-)$ arrangement in which $\mathrm{Pd}$ is connected to negative polarity. The broadness of the XRD peaks is determined by the particle size and lattice imperfections. In the case of mixed phases, the XRD peaks should additionally be broadened, if there is a distribution of compositions rather than one fixed composition. The pure influence of the particle diameter on the peak broadness (full width at half maximum in radians, FWHM, $\beta$ ) is given by the Scherrer equation (Waseda et al., 2004):

$D=\frac{K \lambda}{\beta \operatorname{Cos}\left(\theta_{B}\right)}$ 
where $\lambda$ is $0.154 \mathrm{~nm}$ for the wavelength of $\mathrm{CuK} \alpha$ radiation, $\mathrm{K}$ represents the correction factor for the shape of particles which was considered as 0.9 , and $\theta_{\mathrm{B}}$ the Bragg angle of the reflection that is analyzed. The calculated particle sizes from the reflections of the 220 and 311 planes were around 5 and $6 \mathrm{~nm}$ in agreement with the micrographs. This may indicate that the broadness of peaks is mainly due to particle size. The peaks corresponding to the initial materials of $\mathrm{Au}$ and $\mathrm{Pd}$ were much sharper than the peaks of the mixed phase and consistent with the sizes of the round large particles (see micrograph in Figure 4. 5). As we regard these particles as solidified droplets coming from a certain electrode, this also explains that they are not or hardly mixed.

Regarding the surface area measurements listed in Table 4.5, it was noticeable that the BET surface area of the pure gold and palladium were around $14 \mathrm{~m}^{2} / \mathrm{g}$, and $58 \mathrm{~m}^{2} / \mathrm{g}$ respectively, while the specific surface area of the mixed particles was larger (around $67 \mathrm{~m}^{2} / \mathrm{g}$ ).

Table 4.5 Specific surface area of Au-Pd nanoparticles

\begin{tabular}{|l|l|l|l|}
\hline Electrode & $\begin{array}{l}\text { BET surface area } \\
\left(\mathrm{m}^{2} / \mathrm{g}\right)\end{array}$ & $\begin{array}{l}\text { Calculated BET } \\
\text { diameters }(\mathrm{nm})\end{array}$ & $\begin{array}{l}\text { TEM mean } \\
\text { diameter }(\mathrm{nm})\end{array}$ \\
\hline $\mathrm{Au}-\mathrm{Pd}$ & 67 & $5-7$ & 6 \\
\hline $\mathrm{Au}$ & 14 & 22 & 5 \\
\hline $\mathrm{Pd}$ & 58 & 9 & 4 \\
\hline
\end{tabular}

The particle size distributions, measured by mobility analysis are shown in Figure 4.8.The modal particle sizes from the DMA measurements are in the size range of the particles in the TEM micrographs indicating that there was little agglomeration of the primary particles on the way to the DMA, and the peak sizes can be regarded as approximations of the primary particle sizes. The particle size distribution of the $\mathrm{Au}(+) \mathrm{Pd}(-)$ arrangement with $\mathrm{Pd}$ as the cathode, is closer to the $\mathrm{Pd}$ particle size distribution. This may be due to a larger content of $\mathrm{Pd}$ in the $\mathrm{Au}(+) \mathrm{Pd}(-)$ arrangement.

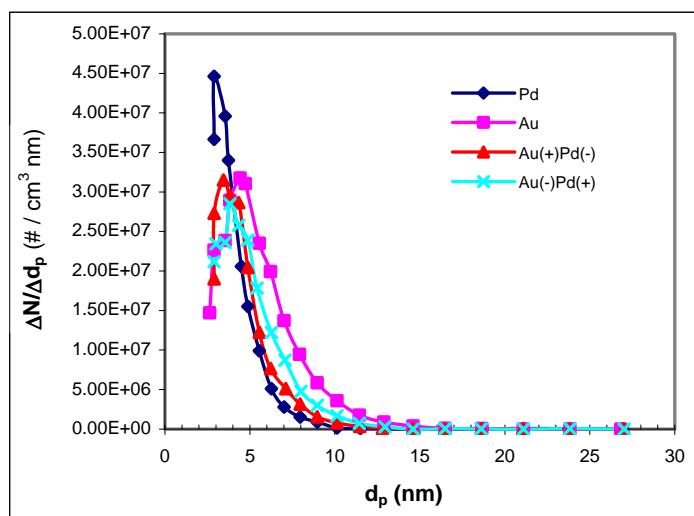

Figure 4.8 Size distributions of $\mathrm{Au}-\mathrm{Pd}$ nanoparticles $(C=5 \mathrm{nF}, d=1 \mathrm{~mm}, f=10 \mathrm{~Hz}, Q=5 \mathrm{lpm} \mathrm{Ar}$ )

\subsubsection{Ag-Pd}

TEM and STEM images of Ag-Pd particles are shown in Figure 4.9. The particle sizes were a few nanometres. These particles showed less sintering (neck formation) and apparently no cold sintering. Neck formation is driven by surface free energy reduction and the initial step of inter-particle diffusion requires completely clean surfaces. The presence of Pd seems to hinder sintering, and this may be due to a larger tendency of chemisorption of gas impurities like oxygen. 

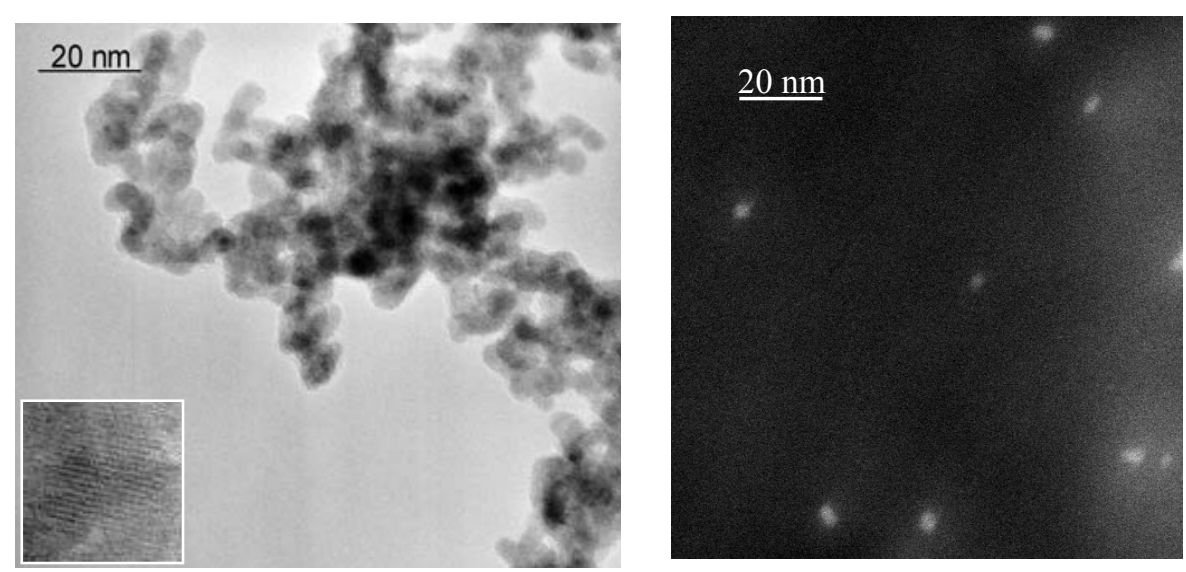

Figure 4.9 TEM micrograph (left) and STEM image from less concentrated region of the sample (right)

Average composition of the particles measured by ICP is listed in Table 4.6. The weight ratio of $\mathrm{Ag} / \mathrm{Pd}$ when $\mathrm{Ag}$ was connected to the negative polarity was larger, in agreement with the results for $\mathrm{Au}-\mathrm{Pd}$, and again, the reason is stronger ablation of the cathode.

Table 4.6 Average composition of Ag-Pd samples measured by ICP

\begin{tabular}{|l|l|}
\hline Electrodes & $\mathrm{Ag} / \mathrm{Pd}$ weight ratio in the sample \\
\hline $\mathrm{Ag}(-) \operatorname{Pd}(+)$ & 0.70 \\
\hline $\mathrm{Ag}(+) \operatorname{Pd}(-)$ & 0.59 \\
\hline
\end{tabular}

Ongoing separate studies have shown that damping the circuit and thus decreasing voltage reversal further reduces erosion of the anode. Thus we conclude that our design of the circuit to exhibit voltage reversal is important for effective mixing to occur.
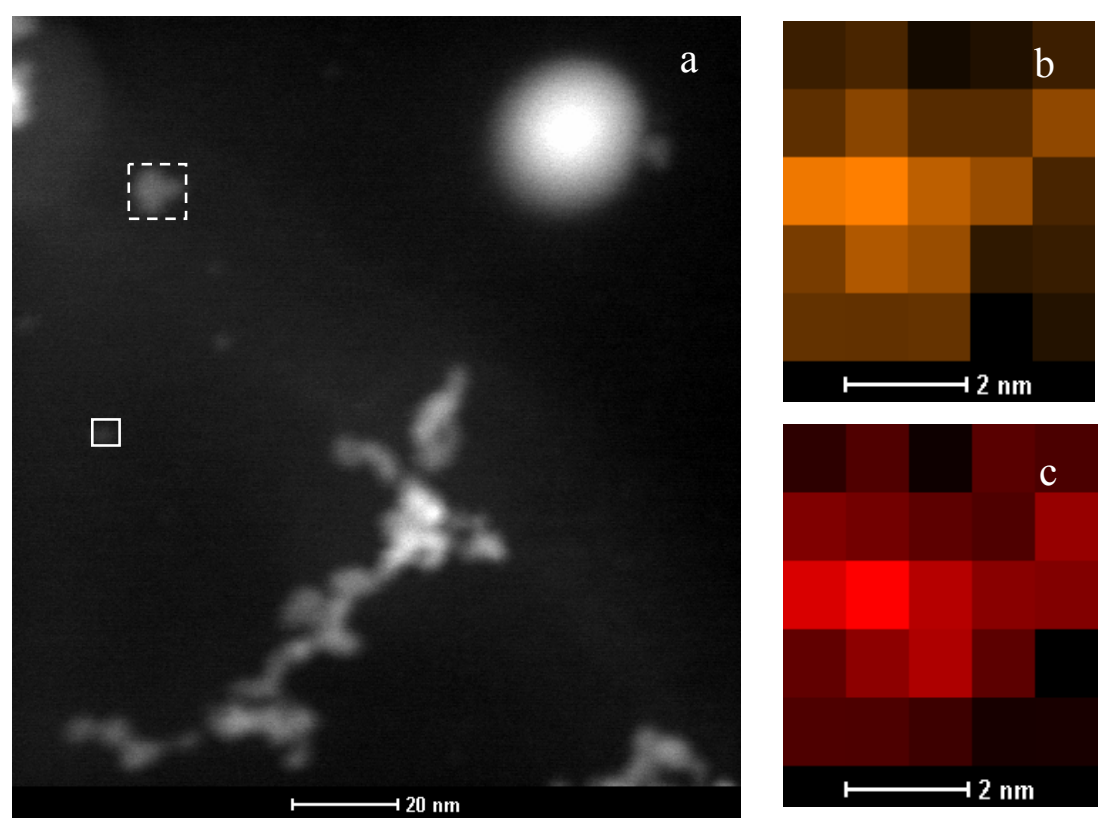

Figure 4.10 Typical STEM maps of a Ag-Pd particle (a): AgL, (b): PdL, (c) 
A typical elemental map of a small particle can be seen in Figure 4.10. The intensities of the $\mathrm{Ag}$ and Pd signals reveal no phase segregation on the surface, just as in the case of Au-Pd. The EDS spectrum in Figure 4.11 shows that $\mathrm{Ag}$ and $\mathrm{Pd}$ present very close but distinguishable peaks. $\mathrm{Ni}, \mathrm{Si}$ and $\mathrm{O}$ are also detected. The former is the constituent of the grid while Si is an impurity frequently found. The oxygen may be due to physisorption on the carbon coated grid and/or chemisorption on the particle surfaces.

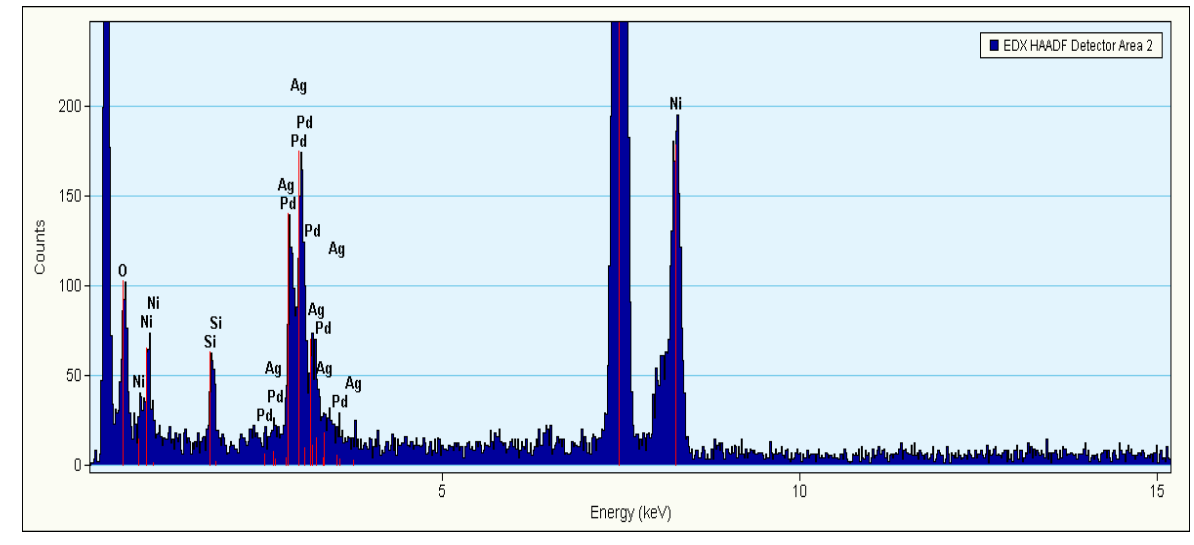

Figure 4.11 Typical EDS spectrum of a Ag-Pd particle

In order to study the composition of individual particles, many particles were analyzed by EDS. The compositions indicated in Table 4.7 show a large variation. As in the case of Au$\mathrm{Pd}$, this can be attributed to inhomogeneous mixing of the vapors in the spark evaporation process.

Table 4.7 Compositional analysis of seven individual nanoparticles $[A g(-) P d(+)]$

\begin{tabular}{|c|c|c|c|c|c|c|c|c|}
\hline \multicolumn{7}{|c|}{$\mathrm{Ag} / \mathrm{Pd}$ weight ratio } & Average & Std \\
\hline 0.954785 & 1.325929 & 3.237425 & 5.088961 & 2.593984 & 0.555848 & 1.12213 & 2.12558 & 1.618559 \\
\hline
\end{tabular}

X-ray diffraction patterns of $\mathrm{Ag}, \mathrm{Pd}$ and the mixed particles produced from the $\mathrm{Ag}(+) \mathrm{Pd}(-)$ and $\operatorname{Ag}(-) \mathrm{Pd}(+)$ arrangements are shown in Figure 4.12. Similar to palladium, silver also has fcc structure with a cell constant of $0.40853 \mathrm{~nm}$. According to the phase diagram of Ag-Pd, these metals can form a continuous solid solution (Hansen, 1958). The formation enthalpy of the solid solutions with the compositions listed in Table 4.6 are around -4.1 and $-4.5 \mathrm{~kJ} / \mathrm{g}-$ atom (at $1200^{\circ} \mathrm{k}$ ) (Predel et al., 1998; Gale et al., 2004). For both samples, the formation of alloy phases was confirmed by Bragg reflections situated between those of pure Ag and Pd. Similar to the Au-Pd system, peaks corresponding to the initial constituents of $\mathrm{Ag}$ and $\mathrm{Pd}$ were also observed in addition to the mixed phases. According to the lattice parameter data, the weight ratio of $\mathrm{Ag} / \mathrm{Pd}$ for the $\mathrm{Ag}(-) \mathrm{Pd}(+)$ sample was calculated fromVegard's law to be around 0.7 .

The particle sizes calculated from reflections of 220 planes using the Scherrer equation were around 4-5 nm, in good agreement with the electron microscopy measurements. The sharpness of Bragg reflections for pure Ag in Figure 4.12 may again be due to cold sintering of the pure silver nanoparticles and possibly the presence of some big particles formed from droplets as seen in Figure 4.5 for Au-Pd. 


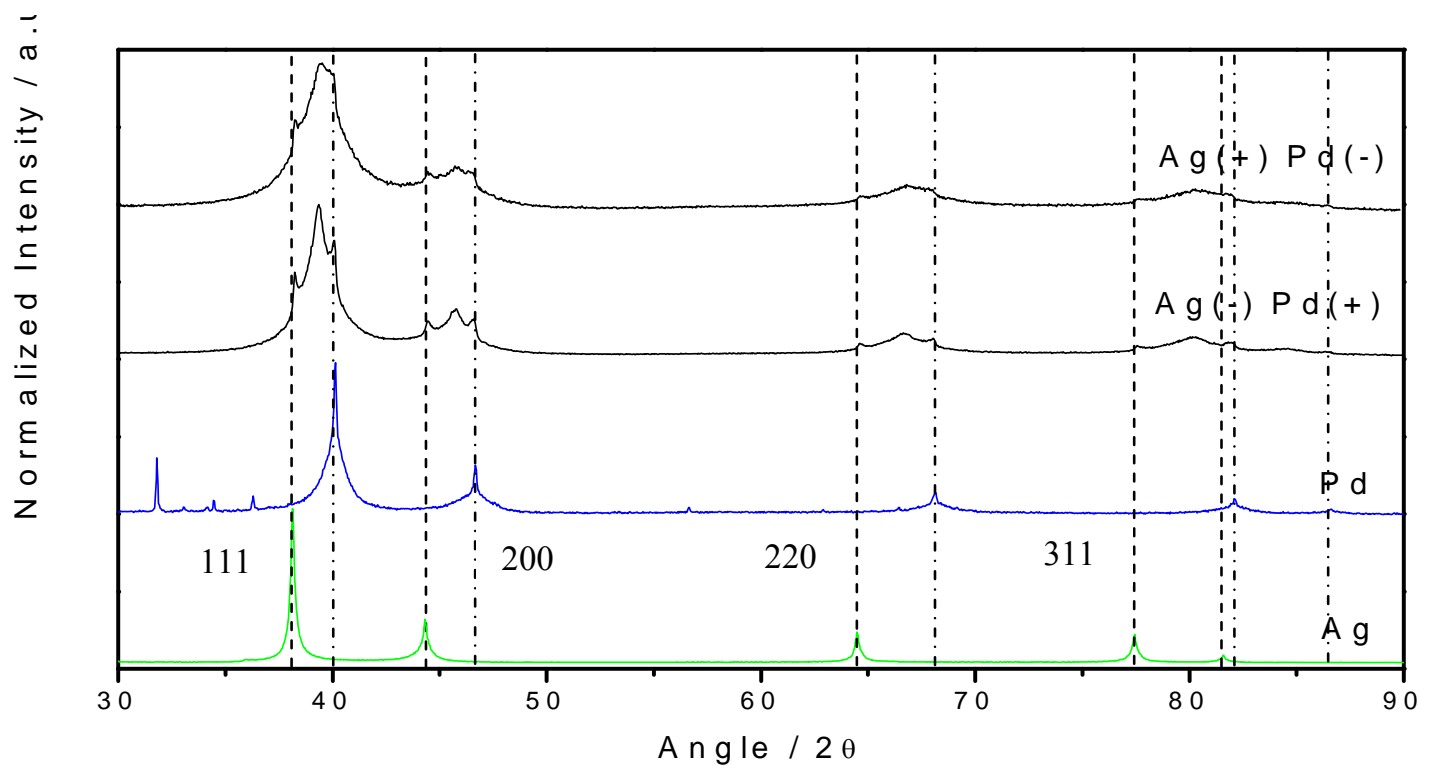

Figure 4.12 XRD patterns of Ag-Pd mixed nanoparticles

The BET specific surface area of the Ag-Pd particles listed in Table 4.8 is around $73 \mathrm{~m}^{2} / \mathrm{g}$ which is quite larger than the specific surface area of the pure Ag or pure $\mathrm{Pd}$. As for $\mathrm{Au}$, this indicates that mixing with $\mathrm{Pd}$ leads to suppression of sintering.

Table 4.8 Specific surface area of Ag-Pd nanoparticles

\begin{tabular}{|l|l|l|l|}
\hline Electrode & BET surface area $\left(\mathrm{m}^{2} / \mathrm{g}\right)$ & Calculated BET diameters $(\mathrm{nm})$ & TEM average diameter $(\mathrm{nm})$ \\
\hline $\mathrm{Ag}-\mathrm{Pd}$ & 73 & $7-8$ & 5 \\
\hline $\mathrm{Ag}$ & $<10$ & $>60$ & 4 \\
\hline $\mathrm{Pd}$ & 58 & 9 & 4 \\
\hline
\end{tabular}

Figure 4.13 shows the size distributions of the Ag and Pd together with the Ag-Pd mixed nanoparticles. All the size distributions are close together with modal diameters between 3-4 $\mathrm{nm}$, in qualitative agreement with the electron microscopy analysis.

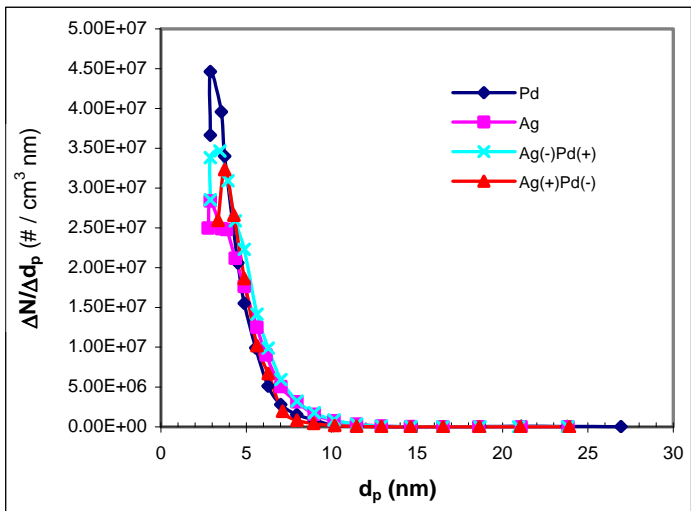

Figure 4.13 Size distributions of mixed Ag-Pd nanoparticles $(C=5 \mathrm{nF}, d=1 \mathrm{~mm}, f=10 \mathrm{~Hz}, Q=5 \mathrm{lpm} \mathrm{Ar})$ 


\subsubsection{Effect of Various Operating Parameters on Mixing}

In order to study the effect of operating parameters on the mixing properties of the spark discharge, a palladium electrode was connected to the negative polarity and a gold electrode was connected to the positive polarity. One parameter at a time was varied, while keeping others constant, and the particles produced were collected and analyzed for their compositions, structures, and phases.

\subsubsection{Effect of Capacitance}

X-ray diffraction patterns of particles produced at various capacitances are shown in Figure 4.14. The Bragg reflections are situated between the characteristic peaks of pure $\mathrm{Au}$ (dash lines) and pure $\mathrm{Pd}$ (dash-dot lines) indicating the formation of intermediate phases. In addition to the mixed phases, pure metals can also be detected. The sharp peaks reflected from the pure metals are likely induced by large solidified droplets sputtered from the electrodes. These large particles are mainly gold. The modes of the mixed nano phases are located approximately in the mid point between pure $\mathrm{Au}$ and $\mathrm{Pd}$ peaks. $\mathrm{Pd} / \mathrm{Au}$ at. $=1$ corresponds to $\mathrm{Pd} / \mathrm{Au}$ wt. $=0.53$. The average compositions of the samples measured by ICP (Table 4.9) are consistent with the composition of the nano mixed phases (Figure 4.14). It may imply that the pure phase of gold weighs around twice of the pure phase of palladium in the samples.

Furthermore, changing the spark energy (via capacitance) within this range does not show significant effect on the relative composition of the constituents in the produced samples.

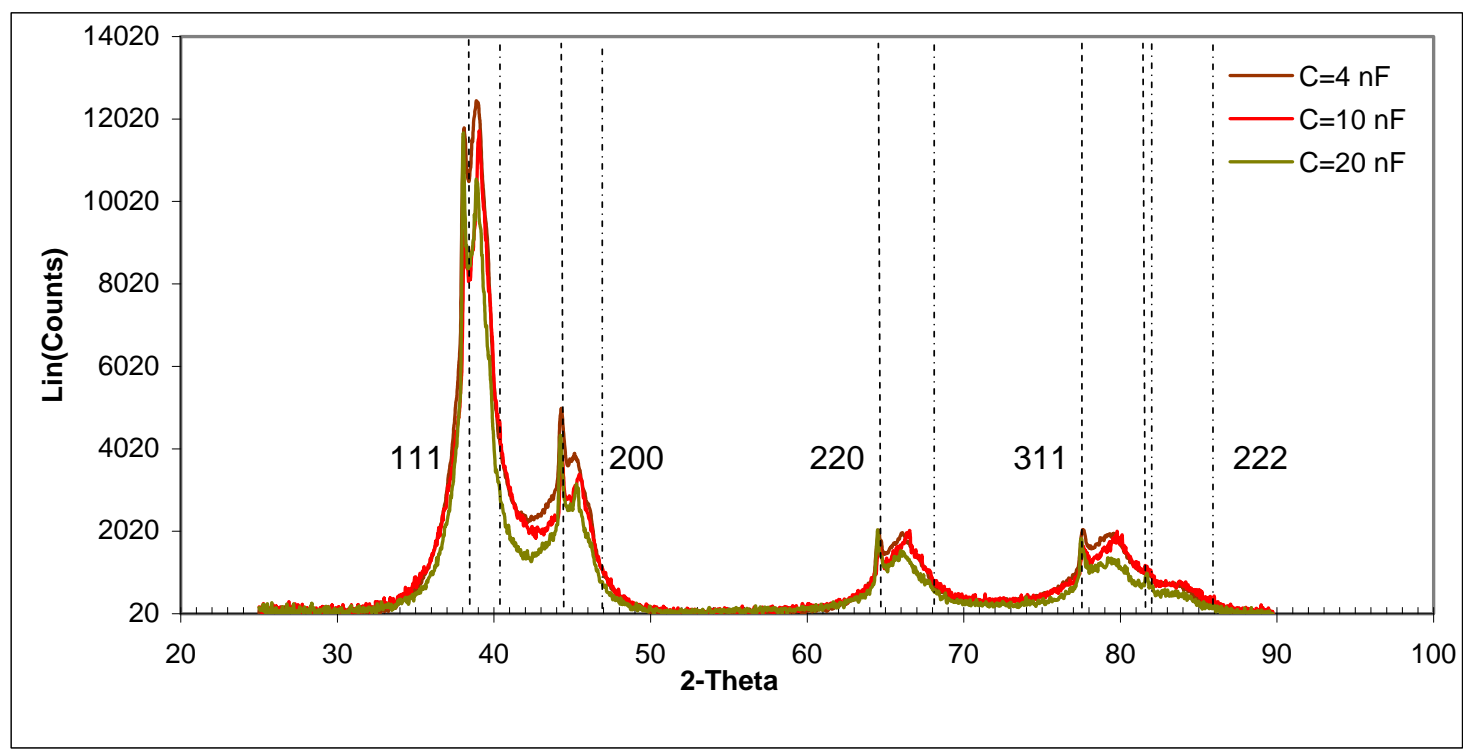

Figure 4.14 XRD patterns of the mixed phases at various capacitances $(d=1 \mathrm{~mm}, f=100 \mathrm{~Hz}, Q=0.8 \mathrm{lpm} \mathrm{Ar})$

Table 4.9 Composition of samples at various capacitances measured by ICP

\begin{tabular}{|l|l|}
\hline Capacitance $(\mathrm{nF})$ & $\mathrm{Pd} / \mathrm{Au}$ (weight ratio) \\
\hline 4 & 0.50 \\
\hline 10 & 0.49 \\
\hline 20 & 0.50 \\
\hline
\end{tabular}




\subsubsection{Effect of Gap Distance}

Formation of mixed phases at various gap distances between the electrodes leads to the XRD patterns in Figure 4.15. Again sharp peaks corresponding to the big particles of pure metals are present.

Table 4.10 indicates that the average $\mathrm{Pd} / \mathrm{Au}$ ratio in the samples decreases as the gap distance increases. These results are consistent with XRD, since the peaks corresponding to the $d=2$ $\mathrm{mm}$ are closer to the $\mathrm{Au}$ reflections implying more $\mathrm{Au}$ in the mixed phase. Figure 4.15 suggests that a pure nano phase of gold may exist in the sample prepared at $\mathrm{d}=0.5 \mathrm{~mm}$ gap. At this gap distance, the peaks are broader probably because of smaller particle sizes. At $d=2$ $\mathrm{mm}$ gap distance which induces a large spark energy, the intensities of the sharp gold peaks, reflected from solidified droplets, are higher. This may partly explain the decreasing trend of the $\mathrm{Pd} / \mathrm{Au}$ weight ratios in Table 4.10.

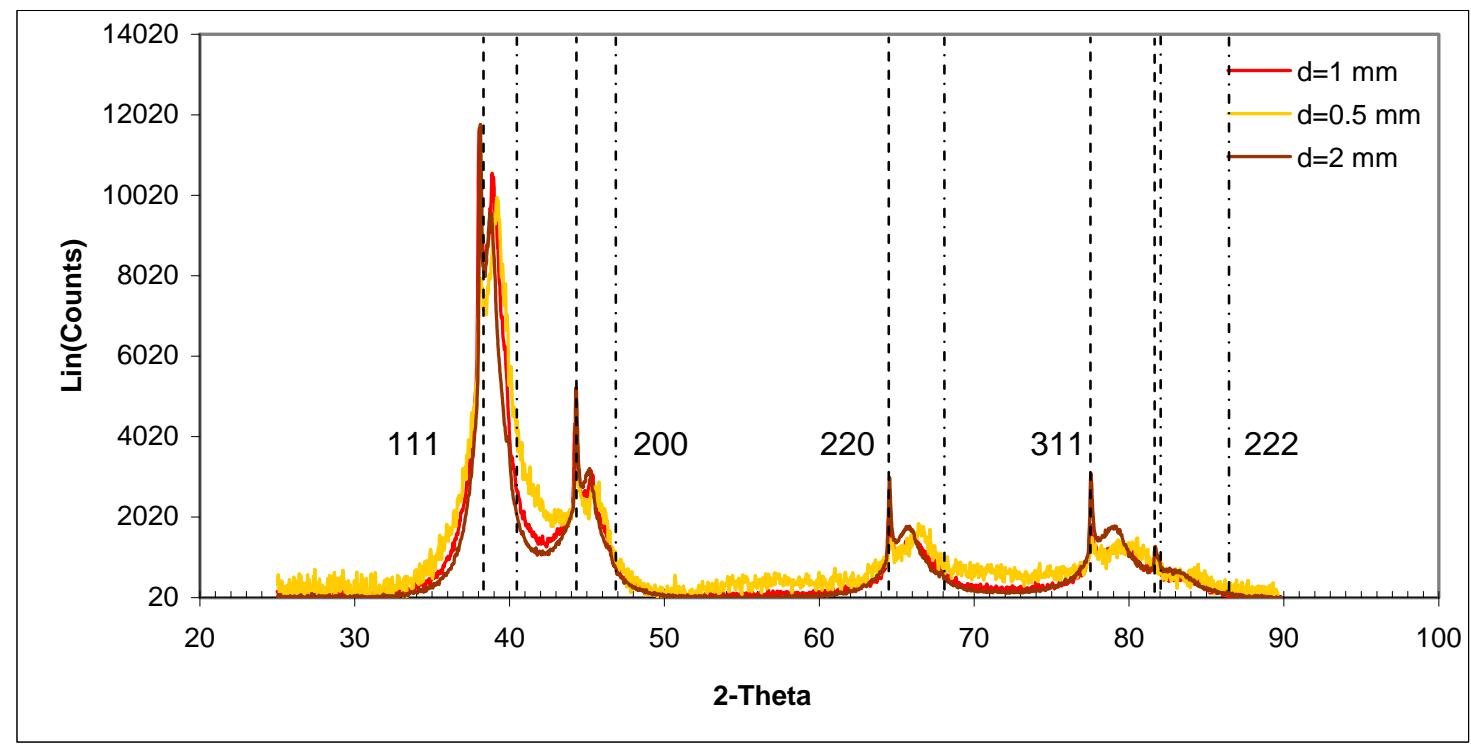

Figure 4.15 XRD patterns of the mixed phases at various gap distances $(C=20 \mathrm{nF}, f=100 \mathrm{~Hz}, Q=0.8 \mathrm{lpm} \mathrm{Ar})$

Table 4.10 Composition of samples at various gap distances measured by ICP

\begin{tabular}{|l|l|}
\hline Gap distance $(\mathrm{mm})$ & $\mathrm{Pd} / \mathrm{Au}$ (weight ratio) \\
\hline 0.5 & 0.54 \\
\hline 1 & 0.50 \\
\hline 2 & 0.49 \\
\hline 3 & 0.37 \\
\hline
\end{tabular}

\subsubsection{Effect of Spark Frequency}

The XRD patterns for various spark repetition rates are shown in Figure 4.16. Again the maxima of the mixed nanophases are positioned around the mid point between $\mathrm{Au}$ and $\mathrm{Pd}$ and the reflections are close with rather similar width and height. Table 4.11 lists the average $\mathrm{Pd} / \mathrm{Au}$ weight ratios in the samples which increase somewhat with the frequency. It may be attributed to the material transfer, which can be enhanced with frequency. The transferred palladium partially covers the gold electrode surface, suppressing the contact of gold with the plasma. 


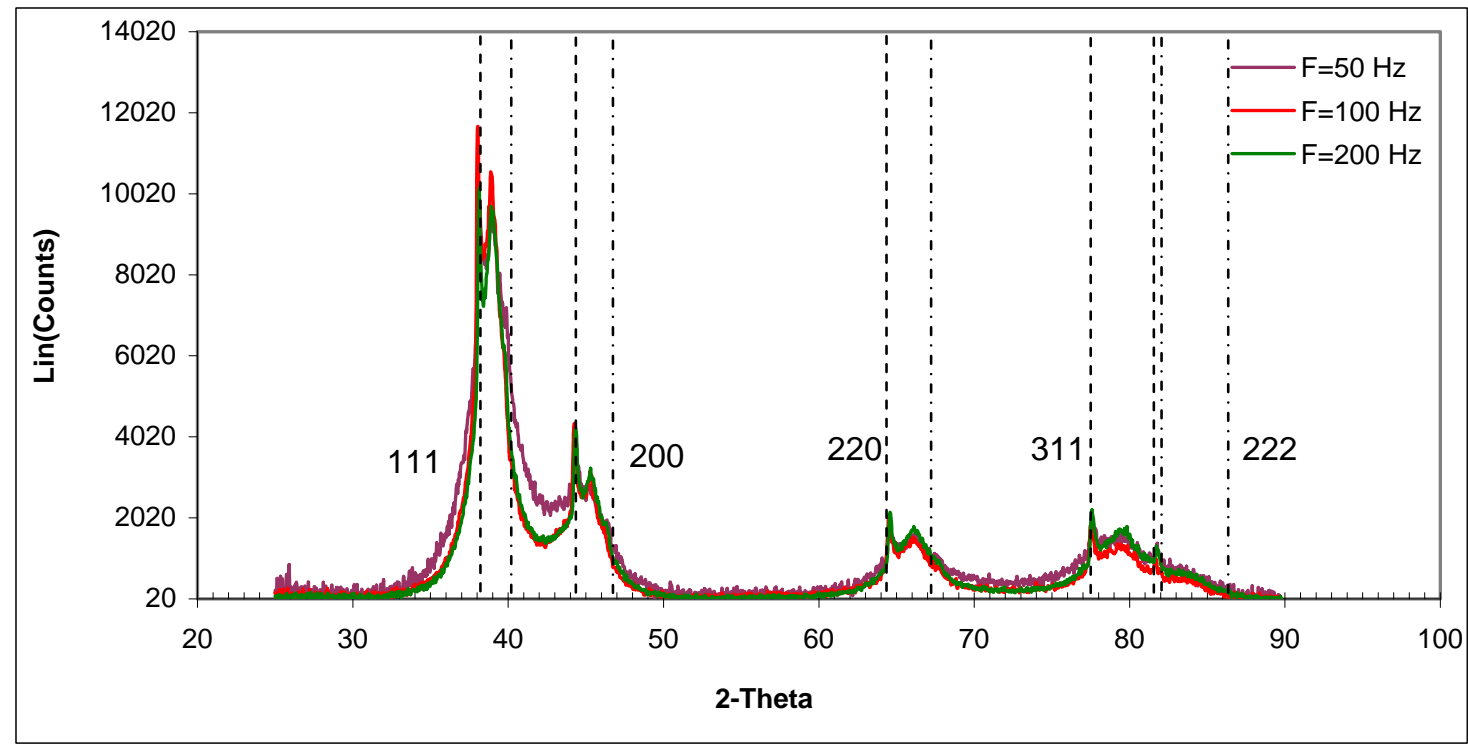

Figure 4.16 XRD patterns of the mixed phases at various frequencies $(d=1 \mathrm{~mm}, C=20 \mathrm{nF}, Q=0.8 \mathrm{lpm} \mathrm{Ar}$ )

Table 4.11 Composition of samples at various frequencies measured by ICP

\begin{tabular}{|l|l|}
\hline Frequency $(\mathrm{Hz})$ & $\mathrm{Pd} / \mathrm{Au}$ (weight ratio) \\
\hline 50 & 0.44 \\
\hline 100 & 0.50 \\
\hline 200 & 0.55 \\
\hline
\end{tabular}

\subsubsection{Effect of Gas Type}

Figure 4.17 shows the intermediate phases formed under $\mathrm{Ar}$ and $\mathrm{N}_{2}$ atmospheres. For the sample produced under nitrogen, a pure gold nanophase is most likely present which is more evident from 200 plane reflection. The average compositional analysis of the samples exhibits almost equal atomic ratios of the metals (Table 4.12).

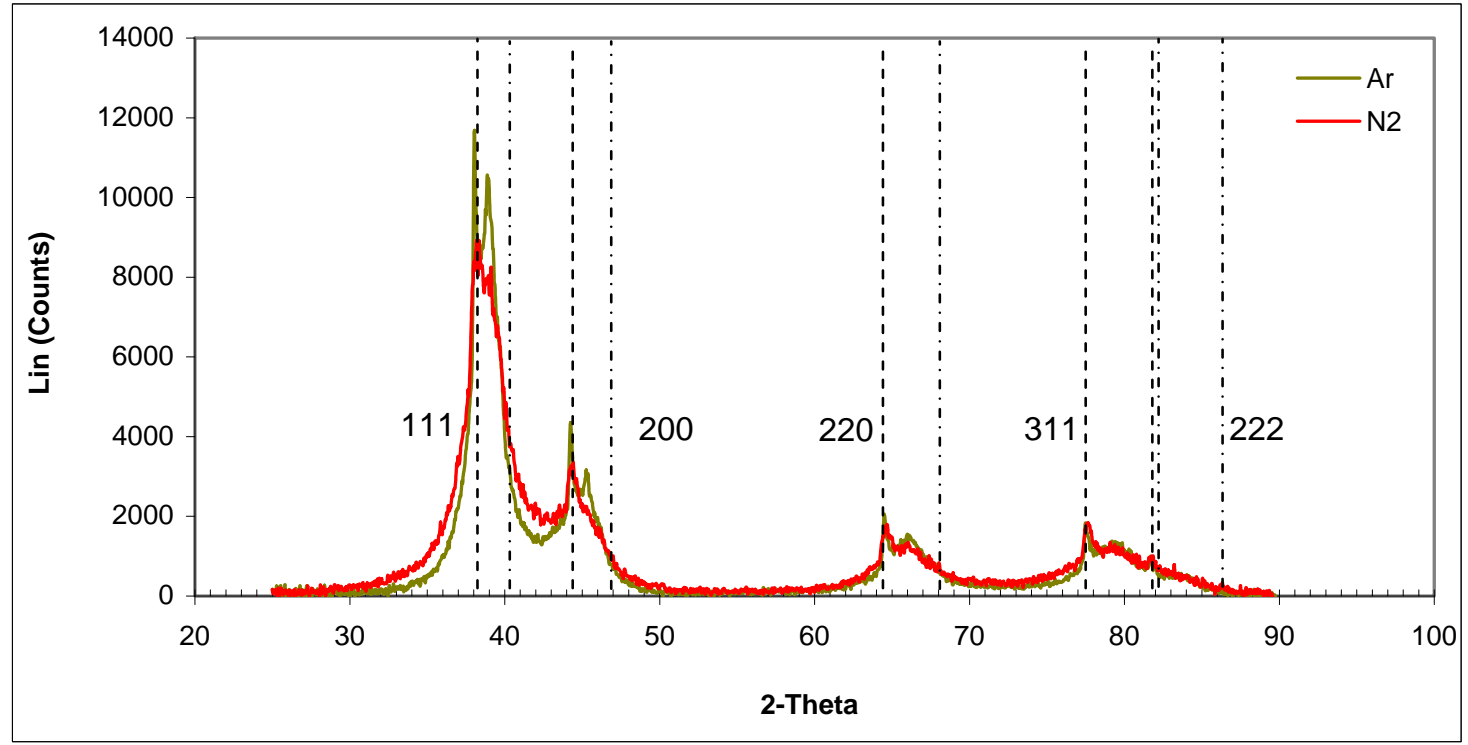

Figure 4.17 XRD patterns of the mixed phases at different gas types (d=1 mm, $C=20 \mathrm{nF}, f=100 \mathrm{~Hz}, Q=0.8 \mathrm{lpm}$ ) 
Since the spark in the nitrogen is more energetic than in the argon, it implies that the variation of the spark energy in this range has no significant impact on the relative erosion of the electrodes, in agreement with the effect of the capacitance (see above).

Table 4.12 Composition of samples at different gas types measured by ICP

\begin{tabular}{|l|l|}
\hline Gas type & $\mathrm{Pd} / \mathrm{Au}$ (weight ratio) \\
\hline $\mathrm{Ar}$ & 0.50 \\
\hline $\mathrm{N}_{2}$ & 0.51 \\
\hline
\end{tabular}

\subsubsection{Effect of Gas Flow Rate}

Figure 4.18 shows that mixed phases are formed at various carrier gas flow rates and the average compositions of the samples are similar as Table 4.13 shows. The peaks corresponding to the lower flow rate $(0.8 \mathrm{lpm})$ are narrower, probably because of the larger particle sizes. In all the cases sharp peaks induced by the large particles of pure constituents (mainly gold) are present. The additional peaks correspond to Si which presents as an impurity in the sample.

Table 4.13 Composition of samples at various gas flow rates measured by ICP

\begin{tabular}{|c|c|}
\hline Flow rate (lpm) & $\mathrm{Pd} / \mathrm{Au}$ (weight ratio) \\
\hline 0.8 & 0.50 \\
\hline 1.4 & 0.49 \\
\hline 2.12 & 0.50 \\
\hline
\end{tabular}

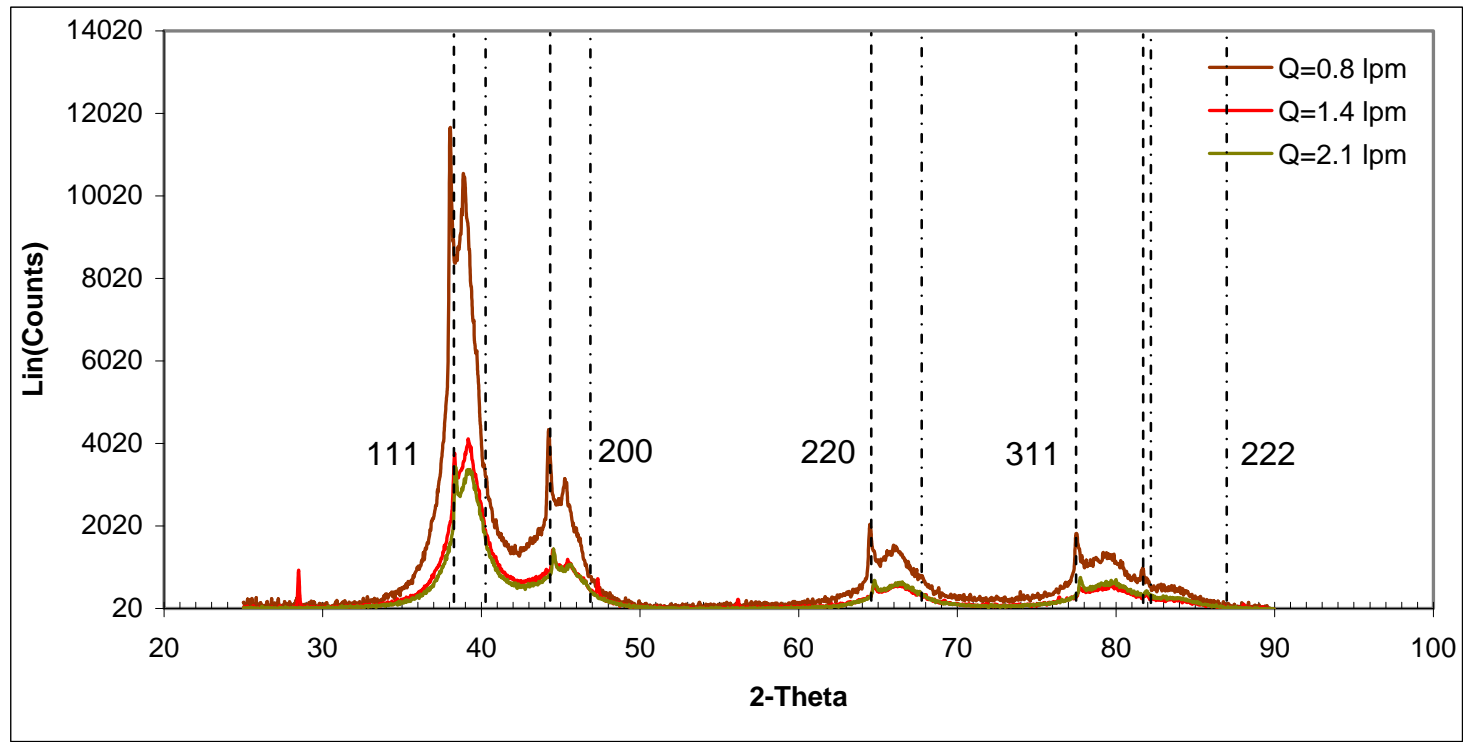

Figure 4.18 XRD patterns of the mixed phases at various gas flow rates (d=1 mm, $C=20 \mathrm{nF}, f=100 \mathrm{~Hz}, \mathrm{Ar}$ ) 


\subsubsection{Compacted electrodes}

In the previous sections mixed phases were generated by using alloyed electrodes or a pair of compositionally different electrodes. However due to a limitation in commercially availability of electrodes, compacted electrodes were fabricated in Nanostructured Materials Group in TUDelft and applied for the first time in the spark discharge generator.

Fabrication of compacted electrodes is performed by Magnetic Pulse Compaction (MPC) in which three Maxwell capacitors are charged to $10 \mathrm{kV}$ to store energy of $90 \mathrm{~kJ}$. This energy is released into a coil via a spark gap in 50-70 $\mu$ s resulting in a current of $300000 \mathrm{~A}$ and a $35 \mathrm{~T}$ magnetic field. The Lorenz forces, induced by the magnetic field, act on the metal tube which is placed inside the coil. The pressure wave (typically 2-5 GPa) leads to compaction of the metal tube (Figure 4.19.a).

The metal tube exposed to the magnetic field is of copper or aluminium of $65 \mathrm{~mm}$ and $32 \mathrm{~mm}$ length, respectively, and a diameter of $21 \mathrm{~mm}$. A polyethylene straw is placed inside. The space between the straw and the metal tube is filled with Polyvinylidene Fluoride. The straw is filled with the powder to be compacted. Then the holder is tapped several thousands of times to reduce the density. The holder is placed inside the coil for compaction (Figure 4.19.b). The composition of the electrode is determined by the mixing ratio of powders. The powder grain size is typically a few micrometers.

In this work many kinds of materials (for example $\mathrm{Sn}-\mathrm{Sb}, \mathrm{Cu}-\mathrm{Sb}$ ) were compacted and transformed into nanoparticles in the spark discharge generator. Various analyses showed that mixed phases were formed when applying compacted electrodes. Some results are published by Lafont et al. (2009).
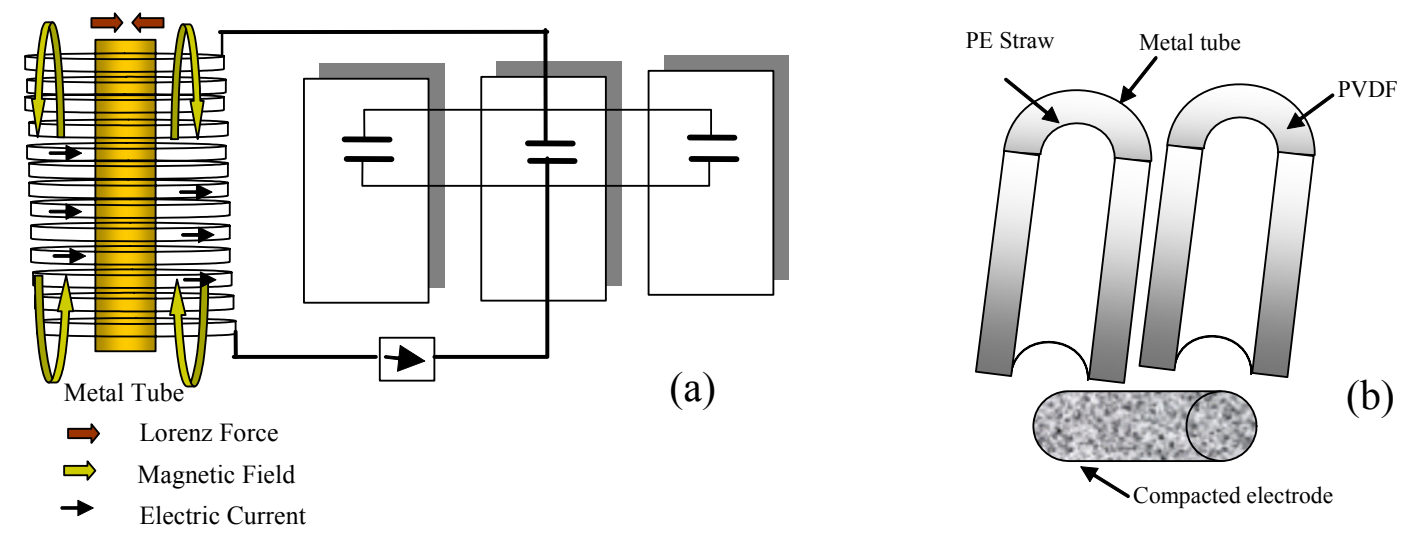

Figure 4.19 Schematic of Magnetic Pulse Compaction (a), and the compacted electrode fabricated by MPC (b)

\subsection{Conclusion}

The spark discharge method can be applied to produce mixed vapors which are very rapidly quenched to form mixed nanoparticles. From an alloyed electrode of $\mathrm{Cr}-\mathrm{Co}$ mixed nanoparticles were produced. The composition of the electrode is more or less reflected in the ratio of the elements in the particulate phase, although the vapor pressures of these materials differ strongly. X-ray diffraction revealed a crystalline mixed Cr-Co phase. 
Effective mixing by the discharge process is observed when using two different electrodes, as shown with the noble metals $\mathrm{Au}-\mathrm{Pd}$ and $\mathrm{Ag}-\mathrm{Pd}$. For this purpose is probably important that the circuit is designed to exhibit an oscillatory discharge. The nanoparticles showed a range of mixing ratios in this case. Following Cundall et al. (1955), the vapor clouds created by the spark expand in the form of jets between the electrodes and if the vapor jets from opposing electrodes meet and mix before condensation, mixed particles can be formed. Thus we expect that the distribution of particle compositions could be narrowed by optimizing the electrode configuration and the electrical circuit characteristics. X-ray diffraction revealed a mixed crystalline phase for both Au-Pd and Ag-Pd. So perfectly mixed rather than layered particles were formed from materials of close evaporation rates (Au-Pd) as well as very different evaporation rates (Ag-Pd). We attribute the latter to fast enough quenching.

The pure $\mathrm{Au}$ and pure Ag particles showed remarkable sintering, which must have occurred at room temperature, indicating extreme purity of the surfaces. Admixing Pd to these metals decreased sintering substantially. This is possibly due to increased chemisorption of gas impurities to the Pd containing particles.

The effect of operating parameters on the mixing of $\mathrm{Au}(+) \mathrm{Pd}(-)$ system was studied and it was revealed that at all the applied conditions a mixed phase was formed and additionally, the initial constituents (mainly gold) were often detected mostly as large solidified droplets sputtered from the liquid pool on the electrode surface.

The mixing ratio was influenced by the polarity and by the gap distance between anode and cathode. Furthermore, the electrical characteristics (for example damping) of the oscillatory discharge is expected to influence the mixing ratio. This remains to be studied.

\section{References}

Barret C. S., et al, The Structure of the Metals, McGraw-Hill, New York, 1996, pp. 372

Byeon J. H., J. H. Park, J. Hwang, Spark Generation of Monometallic and Bimetallic Aerosol Nanoparticles, Journal of Aerosol Science, Volume 39, 2008, pp. 888-896

Considine Douglas M., Van Nostrand's Scientific Encyclopedia, 2002, John Wiley \& Sons, Inc.

Devarajan Supriya, Parthasarathi Bera, S. Sampath, Bimetallic Nanoparticles: A Single Step Synthesis, Stabilization, and Characterization of $\mathrm{Au}-\mathrm{Ag}, \mathrm{Au}-\mathrm{Pd}$, and $\mathrm{Au}-\mathrm{Pt}$ in Sol-Gel Derived Silicates, Journal of Colloid and Interface Science, Volume 290, 2005, pp. 117-129

Evans D. E., the Generation and Characterization of Metallic and Mixed Element Aerosols for Human Challenge Studies, Aerosol Science and Technology, Volume 37, 2003, pp. 975-987

Fernández A. L., Pablo L. D., Formation and the Colour Development in Cobalt Spinel Pigments, Pigments \& Resin Technology, Volume 31, Number 6, 2002, pp. 350-356

Gale W. F., T. C. Totemeier, Smithells Metals Reference Book, ASM International, The Materials Information Society, 2004

Grass Robert N., Wendelin J. Stark, Gas Phase Synthesis of fcc-Cobalt Nanoparticles, Journal of Material Chemistry, Volume 16, 2006, pp. 1825-1830

Hansen Phil. Max, Constitution of Binary Alloys, McGraw-Hill New York, 1958

Hinds William C. , Aerosol Technology, Properties, Behaviors, , and Measurements of Airborne Particles, John Wiley\&Sons, 1999 
Hirakawa Kazutaka, Naoki Toshima, Ag/Rh Bimetallic Nanoparticles Formed by Self-Assembly from Ag and Rh Monometallic Nanoparticles in Solution, Chemistry Letters, Volume 32, Number 1, 2003, pp. 78-79

Hume-Rothery William, G. V. Raynor, the Structure of Metals and Alloys, 1954, the Institute of Metals

Kahng S.-J., Y. J. Choi, J.-Y. Park, and Y. Kuka, Phase Separation in a Two-Dimensional Co-Cr Alloy, Applied Physics Letters, Volume 74, Number 8, 1999, pp. 1087-1089

Keskinen H., J. M. Mäkelä, M. Vippola, M. Nurminen, J. Liimatainen, T. Lepisto, J. Keskinen, Generation of Silver/Palladium Nanoparticles by Liquid Flame Spray, J. Mater. Res., Volume 19, Number. 5, 2004, pp. 15441550

Kim Jong-Taek, Jen-Shih Chang, Generation of Metal Oxide Aerosol Particles by a Pulsed Spark Discharge Technique, Journal of Electrostatics, Volume 63, 2005, pp. 911-916

Kim Min-Joo, Heay-Jin Na, Kyoung Chul Lee, Eun Ah Yoo, Minyung Lee, Preparation and Characterization of $\mathrm{Au}-\mathrm{Ag}$ and $\mathrm{Au}-\mathrm{Cu}$ Alloy Nanoparticles in Chloroform, Journal of Material Chemistry, Volume 13, 2003, pp. 1789-1792

Koch Carl C., Nanostructured Materials, William Andrew, 2007

Kubaschewski O., C. B. Alcock, Metallurgical Thermo-Chemistry, International Series on Materials Science and Technology, Volume 24, Pergamon International Library, 1979

Lafont Ugo, Loic Simonin, Nooshin Salman Tabrizi, Andreas Schmidt-Ott, Erik Kelder, Synthesis of Nanoparticles of $\mathrm{Cu}, \mathrm{Sb}, \mathrm{Sn}, \mathrm{SnSb}$, and $\mathrm{Cu}_{2} \mathrm{Sb}$ by Densification and Atomization Process, Journal of Nanoscience and Nanotechnology, Volume 9, 2009, pp. 2546-2552

Liu H. B., U. Pal, A. Medina, C. Maldonado, J. A. Ascencio, Structural Incoherency and Structure Reversal in Bimetallic Au-Pd Nanoclusters, Physical Review, Volume B 71, 2005, pp. 075403 1-6

Menon Mahesh, Badal C. Khanra, Alloying Behaviour in Cu-Pd Nanostructures, Physica B, Volume 304, 2001, pp. 181-185

Nutt Michael O., Kimberly N. Heck, Pedro Alvarez, Michael S. Wong, Improved Pd-on-Au Bimetallic Nanoparticle Catalysts for Aqueous-Phase Trichloroethene Hydrodechlorination, Applied Catalysis B: Environmental, Volume 69, 2006, pp. 115-125

Okitsu Kenji, Masatoshi Murakami, Shuji Tanabe, Hiroshige Matsumoto, Catalytic Behaviour of Au Core/Pd Shell Bimetallic Nanoparticles on Silica Prepared by Sonochemical and Sol-Gel Processes, Chemistry Letters, 2000, pp. 1336-1337

Ouyang G., X. Tan, C. X. Wang, G. W. Yang, Charge- Induced Transition between Miscible and Immiscible in Nanometer-Sized Alloying Particles, Chemical Physics Letteres, Volume 423, 2006, pp. 143-146

Papageorgiou I., C. Brown, R. Schins, S. Singh, R. Newson, S. Davis, J. Fisher, E. Ingham, C. P. Case, The Effect of Nano-and Micron-Sized Particles of Cobalt-Chromium Alloy on Human Fibroblasts in Vitro, Biomaterials, Volume 28, 2007, pp. 2946-2958

Powell A., J. Van Den Avyle, B. Damkroger, J. Szekely and U. Pal, Analysis of Multicomponent Evaporation in Electron Beam Melting and Refining of Titanium Alloy, Metall. Mater. Trans., Volume 28B, Number 6, 1997 , pp. 1227-1239

Predel B., O. Madelung, Phase Equilibria, Crystallographic and Thermodynamic Data of Binary Alloys, Belrin: Springer, 1998

Regen Maureen R., Ipsita A. Banerjee, Preparation of Au-Pd Bimetallic Nanoparticles in Porous Germania Nanoparticles: a Study of their Morphology and Catalytic Activity, Scripta Materialia, Volume 54, 2006, pp. 909-914 
Rouquerol Francoise, Jean Rouquerol, Kenneth Sing, Adsorption by Powders and Porous Solids: Principles, Methodology and Applications, San Diego Academic Press, 1999

Silvert P. Y., V. Vijayakrishnan, P. Vibert, R. Herrera-Urbina, K. T. Elhsissen, Synthesis and Characterization of Nanoscale Ag-Pd Alloy Particles, Nanostructured Materials, Volume 7, Number 6, 1996, pp. 611-618

Tabrizi N. S., M. Ullmann, V. A. Vons, U. Lafont, A. Schmidt-Ott, Generation of Nanoparticles by Spark Discharge, Journal of Nanoparticle Research, Volume 11, 2009, pp.315-332

Tabrizi N. S., Q. Xu, N. M. van der Pers, U. Lafont, A. Schmidt-Ott, Synthesis of Mixed Metallic Nanoparticles by Spark Discharge, Journal of nanoparticle Research, Volume 11, Number 5, 2009, pp. 1209-1218

Turker Mehmet, Effect of Production Parameters on the Structure and Morphology of Ag Nanopowders Produced by Inert Gas Condensation, Materials Science and Engineering A, Volume 367, 2004, pp. 74-81

Vemury S., S. Pratsinis, Self Preserving Size Distributions of Agglomerates, Journal of Aerosol Science, Volume 26, 1995, pp. 175-185

Wang Kuan-Wen, Shu-Ru Chung, Tsong-Pyng Perng, Surface Segregation and Homogenization of $\mathrm{Pd}_{70} \mathrm{Ag}_{30}$ Alloy Nanoparticles, Journal of Alloys and Compounds, Volume 422, 2006, pp. 223-226

Waseda Y., A. Muramatsu, Morphology Control of Materials and Nanoparticles, Springer, 2004

Xiao S., W. Hu, W. Luo, Y. Wu, X. Li, H. Deng, Size Effect on Alloying Ability and Phase Stability of Immiscible Bimetallic Nanoparticles, The European Physical Journal B, Volume 54, 2006, pp. 479-484

Yang Chia-Cheng, Chi-Chao Wan, Yung-Yun Wang, Synthesis of Ag/Pd Nanoparticles via Reactive Micelles as Templates and its Application to Electroless Copper Deposition, Journal of Colloid and Interface Science, Volume 279, 2004, pp. 433-439

Yang Yonglai, Khaled M. Saoud, Victor Abdelsayed, Garry Glaspell, Sarojini Deevi, M. Samy El-Shall, Vapor Phase Synthesis of Supported Pd, Au, and Unsupported Bimetallic Nanoparticles Catalysts for CO Oxidation, Catalysis Communications, Volume 7, 2006, pp. 281-284

Zhang J. P., D. T. Foord, J. Chou, E. W. McFarland, Direct Evidence of Shelled Structures of Au-Pd Bimetallic Nano-Particles, Microsc Microanal, Volume 11, Supplement 2, 2005, pp. 1468-1469 


\section{Chapter 5}

\section{Generation of Bimetallic Nanoparticles from Immiscible Metals}

\subsection{Introduction}

Flexible mixing of materials on a nano scale down to the atomic scale would give access to an enormous variety of new material properties tunable not only through size but also through mixing ratios and the scale of mixing. This should lead to myriad applications in areas such as optoelectronics, catalysis, batteries, solar cells, fuel cells, hydrogen storage, magnetic materials, and sensors (Wu et al., 2004). In principle, nanomixing offers additional degrees of freedom for tailoring properties to match the application, and pure materials or stochiometric mixing ratios are likely to become exceptions in functional materials of the future, if suitable methods of mixing are found.

There have been many attempts to generate mixed nanoparticles through different methods such as vapour quenching, co-deposition sputtering, mechanical alloying and ion-beam mixing or irradiation (Almtoft et al., 2007; Radic et al., 1998). In the present study we used the spark discharge method to produce bimetallic nanoparticles and focused on immiscible systems to investigate the feasibility of the generation of internally mixed metallic particles from immiscible constituents.

We studied $\mathrm{Ag}-\mathrm{Cu}$, Au-Pt, and $\mathrm{Cu}-\mathrm{W}$ systems which show miscibility gaps on the macroscopic scale and the constituents have very different vapour pressures and evaporation rates (Kubaschewski, 1979; Powell et al., 1997). We expected enhanced solubility firstly because of our synthesis method which involves with the fast quenching of the vapours and secondly because of the size effect of the ultra fine particles.

The presence of a large fraction of atoms composing a nanoparticle at the surface, which contribute to the excess Gibbs free energy may modify the bulk phase diagram of the particulate binary system (Lahiri et al., 2005). In addition, reduction in the melting point of the nanoparticles (Ding et al., 2004) and presence of defects at the interface in bimetallic nanoparticles may enhance the inter-diffusion of the metals and change the alloying characteristics (Lahiri et al., 2005; Birringer, 1989). Using Monte Carlo simulations Christensen et al. (1995) investigated the size dependence of phase separation in small bimetallic solid clusters. For the Ag-Cu system, which shows immiscibility in bulk for a broad range of compositions up to the melting temperature, they found that the maximum temperature where phase separation can occur is strongly size dependent. They also found the absence of phase separation for clusters smaller than a critical size of about 270 atoms. By taking into consideration that electric charge can energetically affect the free energy of the formation of the clusters, Ouyang et al. (2006) proposed a charge-dependent thermodynamic model for nano-sized alloy particles to address the phase transformation between miscible and immiscible. By applying a thermodynamic model and an analytic embedded atom method Xiao et al. (2006) recently showed that the heat of formation of alloy nanoparticles is 
not only composition dependent but also size dependent and for immiscible systems with a positive heat of formation, a negative heat of formation may be gained for alloy nanoparticles of small size or a dilute solute component.

The examples that we chose have interesting applications (Cagran et al., 2005) for instance, the high electrical conductivity of $\mathrm{Ag}$ and low electrical migration of $\mathrm{Cu}$ make $\mathrm{Ag}-\mathrm{Cu}$ nanoparticles suitable as conductive fillers in electrically conductive adhesives (Jiang et al., 2005). Au-Pt nanoparticles have been used in various catalytic reactions (Zeng et al., 2006; Devarajan et al., 2005) and exhibit specific activity and selectivity in hydrogenation (Patel et al., 2005). The high thermal and electrical conductivity of $\mathrm{Cu}$, and low thermal expansion coefficient of $\mathrm{W}$, lead to various applications for this composite as heat sinks, and in microprocessors, microwave modules, wireless telecommunication devices, and other RF packages (MPR 2000; Kang et al., 2003).

\subsection{Experiment}

The experimental setup is described in the previous chapter (4.3). For production of the Ag$\mathrm{Cu}$ and $\mathrm{Cu}-\mathrm{W}$ particles, two pairs of sintered electrodes of $(\mathrm{Ag} 72 / \mathrm{Cu} 28)$ and $(\mathrm{W} 72 / \mathrm{Cu} 28)$ were utilized while for the Au-Pt system, two electrodes of pure $\mathrm{Au}$ and pure $\mathrm{Pt}\left(99^{+} \%\right.$ purity) were applied. The inert gas carrying the particles was $\operatorname{Ar}(99.999 \%$ purity). To investigate the morphology, size, composition, and structure of the particles, TEM, EDS, $\mathrm{XRD}$ analyses were performed on the samples. The average compositions were measured by ICP and the specific surface areas were determined by the BET. On-line particle size distribution measurements were carried out by a home-built scanning mobility particle sizer (SMPS).

\subsection{Results and Discussion}

\subsubsection{Ag-Cu}

TEM images of $\mathrm{Ag}-\mathrm{Cu}$ particles at two magnifications can be seen in Figure 5.1. The particles are crystalline, surrounded by an amorphous layer probably of oxide phase(s), (see the close-up). Neck formation is not observed which points to the absence of sintering and cold coalescence, contrary to the pure silver particles which showed significant sintering. The particles are a few nanometres in size.

Very few particles that are much larger (up to $200 \mathrm{~nm}$ ) are present. The large size difference with respect to the small ones implies a different formation mechanism. We assume that these particles are sputtered from local patches of molten metal at the electrode surface.

EDS analysis reveals that the large particles were almost pure Ag. The average composition of the nanoparticles measured at different regions of the sample is about $30 \mathrm{wt} \% \mathrm{Cu}$ and 70 $\mathrm{wt} \% \mathrm{Ag}$. This agrees with the composition of the electrodes, which is $28 \mathrm{wt} \% \mathrm{Cu}$ and $72 \mathrm{wt} \%$ Ag. It implies that the large particles are rare in the sample. The average composition of the sample analysed by ICP confirms this agreement (see Table 5.1). 

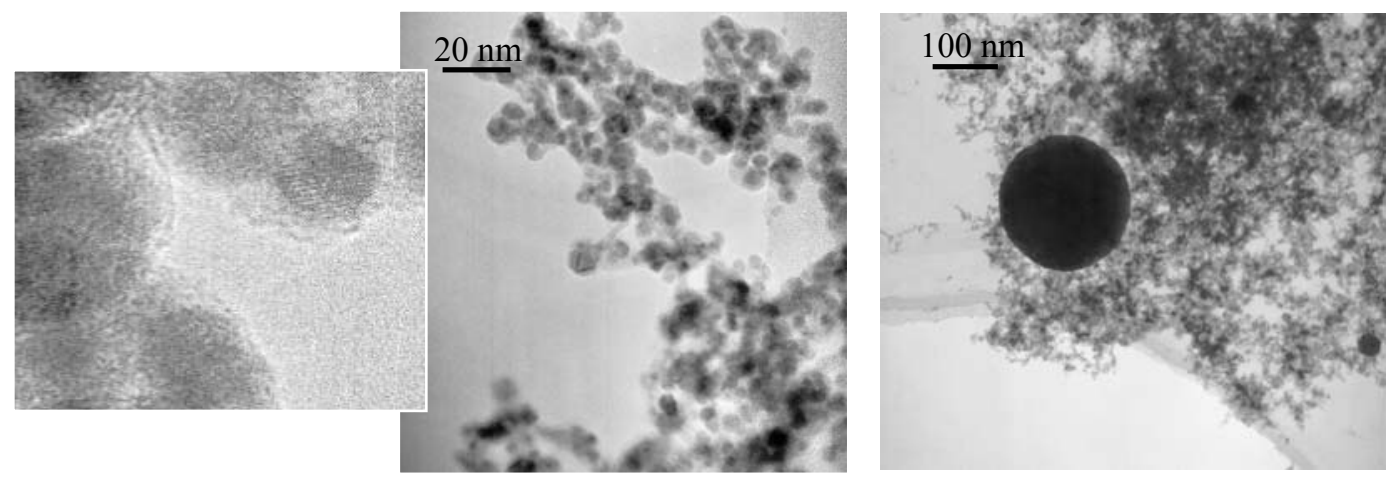

Figure 5.1 Electron micrographs of the Ag-Cu particles

This indicates that there is no preferential evaporation of the more volatile constituents in spite of a significant difference in the boiling points $(2435 \mathrm{~K}$ for $\mathrm{Ag}$ and $3200 \mathrm{~K}$ for $\mathrm{Cu}$ ). Moreover it shows that partial melting of the electrode surface has not distorted the composition at the electrode surface so severely that the lower surface tension material becomes dominant at the surface. The surface free energies of $\mathrm{Ag}$ and $\mathrm{Cu}$ at their melting points are 1.046 and $1.576 \mathrm{~J} \mathrm{~m}^{-2}$ respectively, (Mezey and Giber, 1982).

Table 5.1 Compositional analysis of Ag-Cu sample measured measured by ICP

\begin{tabular}{|l|l|l|}
\hline Material & $\mathrm{Cu} / \mathrm{Ag}$ wt. ratio in the electrode & $\mathrm{Cu} / \mathrm{Ag}$ wt. ratio in the sample \\
\hline Ag-Cu alloyed electrodes & 0.388 & 0.380 \\
\hline
\end{tabular}

X-Ray diffraction pattern of the sample is shown in Figure 5.2. Silver and copper both have fcc (face-centered cubic) structures. In addition to the reflections of Ag, a set of strong fcc Bragg peaks is present, which fits a lattice parameter somewhat smaller than Ag (by a factor of 0.9792). This can be attributed to an $\mathrm{AgCu}$ alloy with $\mathrm{Cu}$ in solid solution with $\mathrm{Ag}$. Moreover there is another set of Bragg reflections corresponding to a lattice parameter slightly larger than pure $\mathrm{Cu}$ (by a factor of 1.0187). This can be ascribed to an $\mathrm{AgCu}$ alloy with $\mathrm{Ag}$ in solid solution with $\mathrm{Cu}$. For $\mathrm{Ag}$ and $\mathrm{Cu}$ the atomic radii are $0.1445 \mathrm{~nm}$ and 0.1278 $\mathrm{nm}$ respectively. Thus $\mathrm{Cu}$ atoms are smaller than $\mathrm{Ag}$ atoms and dissolution of $\mathrm{Cu}$ in the $\mathrm{Ag}$ matrix leads to contraction of the lattice and similarly dissolution of $\mathrm{Ag}$ in $\mathrm{Cu}$ matrix leads to expansion of the lattice (Ceylan et al., 2006). Using the phase diagram of $\mathrm{Ag}-\mathrm{Cu}$ and applying the data for the lattice parameters (Predel et al., 1998), the $\mathrm{AgCu}$ alloys in the sample are estimated to contain about 21.5 at \% $\mathrm{Cu}$ in $\mathrm{Ag}$ (a hypoeutectic alloy) and about 12 at \% $\mathrm{Ag}$ in $\mathrm{Cu}$ (a hypereutectic alloy). According to the phase diagram of the macroscopic $\mathrm{Ag}-\mathrm{Cu}$ system, the maximum solubility of $\mathrm{Cu}$ in $\mathrm{Ag}$ is around 14.1 at. $\%$ and the maximum solubility of $\mathrm{Ag}$ in $\mathrm{Cu}$ is about 4.9 at $\%$ at the eutectic temperature of $1052 \mathrm{~K}$. These results show that the alloying behaviour is significantly modified in our nano-scale system. This is in agreement with the theoretical study carried out by Hajra and Acharya (2004) who found a remarkable decrease in the melting points of the metals, and the solid-solid, and solid-liquid transition and the eutectic temperature with a consequent increase in the solubilities of the nano terminal phases of the Ag-Cu system.

For the above mentioned compositions of the Ag-Cu alloys, the heats of formation at $700 \mathrm{~K}$ are positive, around $4.1 \mathrm{~kJ} / \mathrm{mol}$ and $2.8 \mathrm{~kJ} / \mathrm{mol}$, respectively (Najafabadi et al., 1993). The sharp silver reflections are explained by the big particles mentioned above, one of which is shown in the TEM micrograph of Figure 5.1. 


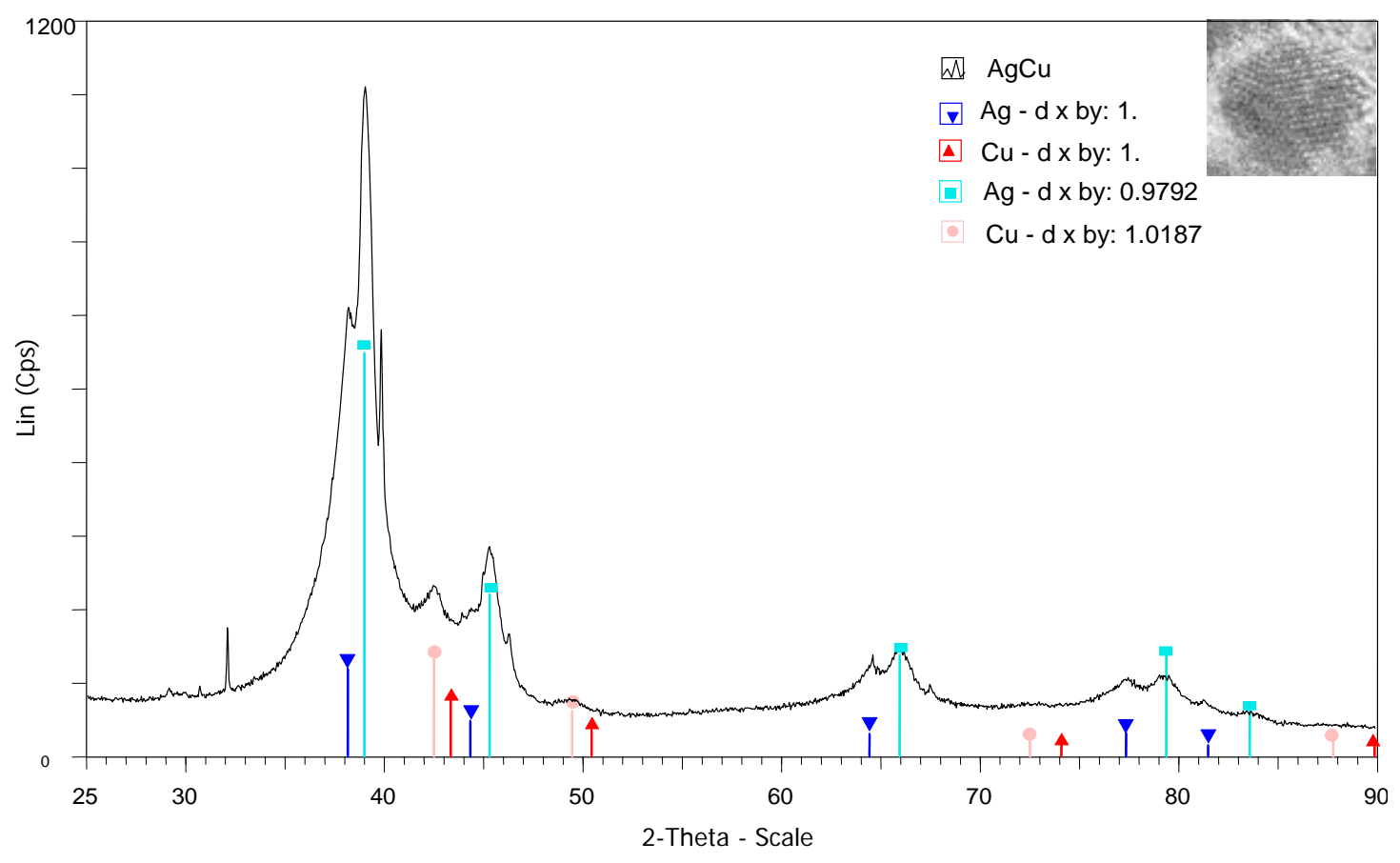

Figure 5.2 X-ray diffraction pattern of Ag-Cu sample

The broadness of $\mathrm{Ag}-\mathrm{Cu}$ reflections is due to nano-sized particles. We do not see characteristic peaks corresponding to crystalline oxide phases. This is probably due to the fact that the oxide phase, covering the particle surfaces as observed in the electron micrographs is amorphous. The undefined reflections are attributed to unknown impurities.

The specific surface areas of $\mathrm{Cu}, \mathrm{Ag}$ and $\mathrm{Ag}-\mathrm{Cu}$ samples measured by the BET method (Rouquerol et al., 1999) and the corresponding BET diameters calculated for spherical shape (shape factor 6) are listed in Table 5.2. Copper nanoparticles present a rather large surface area, consistent with the particle size that we have previously observed in TEM micrographs of pure copper. This may be due to the role that oxygen plays in stopping coalescence. Silver presents a small specific surface area. The discrepancy between the pure silver primary particle size and the large calculated BET diameter can be attributed to the cold sintering and coalescence of silver particles on the substrate, as has previously been observed for pure gold nanoparticles (Tabrizi et al., 2009). The Ag-Cu particles show a similar BET diameter as the $\mathrm{Cu}$ particles, indicating that the presence of $\mathrm{Cu}$ in the particles predominantly consisting of Ag hinders sintering.

Table 5.2 Specific surface area of the Ag-Cu particles

\begin{tabular}{|c|c|c|}
\hline Material & BET specific surface area $\left(\mathrm{m}^{2} / \mathrm{g}\right)$ & Calculated BET diameter $(\mathrm{nm})$ \\
\hline $\mathrm{Ag}$ & $<10$ & $>57$ \\
\hline $\mathrm{Cu}$ & 130 & 5.1 \\
\hline $\mathrm{Ag}-\mathrm{Cu}$ & 97 & 6.4 \\
\hline
\end{tabular}

Size distributions of $\mathrm{Cu}, \mathrm{Ag}$ and $\mathrm{Ag}-\mathrm{Cu}$ particles were measured on-line by SMPS. It can be seen in Figure 5.3, that the produced aerosols contain very small particles of 4-5 nm modal diameters. For $\mathrm{Cu}$ and $\mathrm{Cu}-\mathrm{Ag}$ this is in fair agreement with the BET diameters, while the discrepancy for Ag confirms the cold sintering on the substrate. 


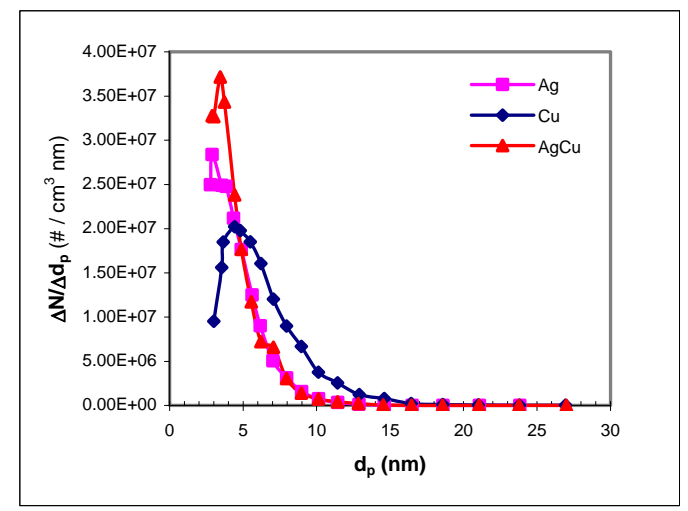

Figure 5.3 Size distributions of $\mathrm{Ag}, \mathrm{Cu}$ and $\mathrm{Ag}-\mathrm{Cu}$ nanoparticles $(C=5 \mathrm{nF}, d=1 \mathrm{~mm}, f=10 \mathrm{~Hz}, Q=5 \mathrm{lpm} \mathrm{Ar}$ )

\subsubsection{Au-Pt}

Electron micrographs of pure $\mathrm{Au}$ and pure Pt presented in Figures 5.4.a, b, show different morphologies of the particle assemblies. Gold particles have significantly sintered on the contact points, and platinum particles seem to form agglomerate with some particles interconnected. Au-Pt particles produced from $\mathrm{Au}$ and Pt electrodes are depicted in Figure (5.4.c). The unattached primary particles are rather spherical and a few nanometers in diameter. They represent the primary particle sizes typically produced by our spark process. In some regions the particles are sintered and joined by necks. Neck growth generally occurs by surface diffusion, dislocation motion and grain rotation of clean particles (Koch, 2007). The tendency of sintering is highest in noble metallic nanoparticles (in particular gold) because of their clean surfaces, since any oxide layer represents a barrier for sintering.
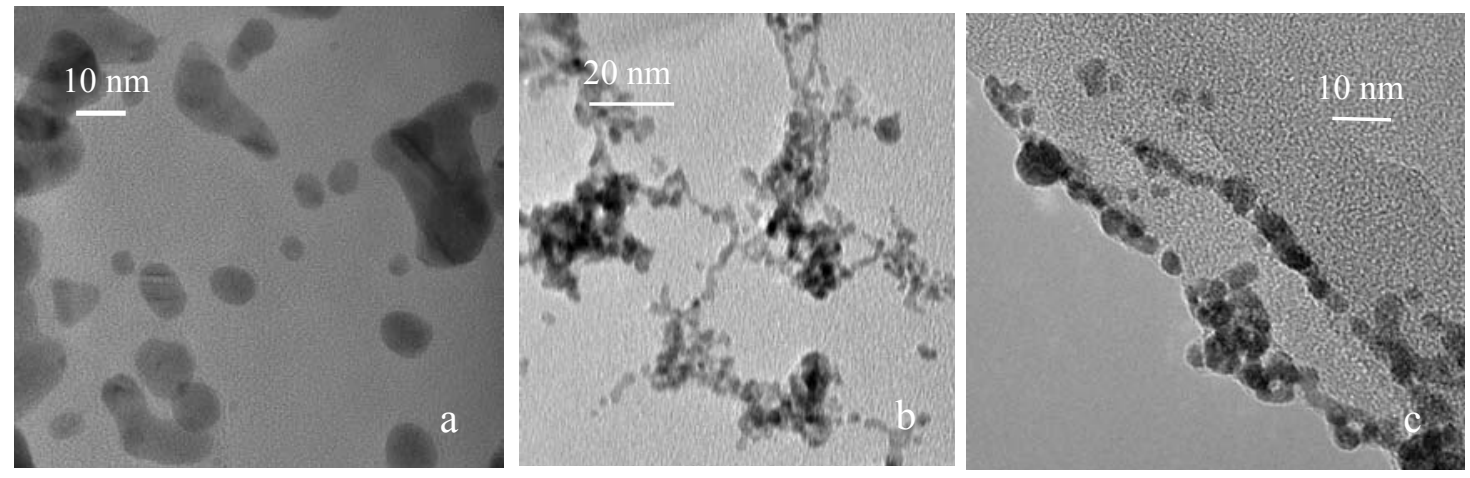

Figure 5.4 TEM images of $A u(a)$, Pt (b), and Au-Pt (c) nanoparticles

In order to study the composition of the particles, a number of single particles in a narrow size range $(5-12 \mathrm{~nm})$ were analyzed by EDS. Table 5.3 lists the elemental compositions of nine particles with an average $\mathrm{Au} / \mathrm{Pt}$ weight ratio of 0.97 . The range of $\mathrm{Au}$ compositions in the measured particles is $22-65$ wt. \%. According to the bulk phase diagram of the Au-Pt system, this compositional range falls well inside the two-phase region at ambient temperature (see Appendix E).

Table 5.3 EDS compositional analysis of single particles [Au(+)Pt(-)]

\begin{tabular}{|l|l|l|l|l|l|l|l|l|l|l|}
\hline \multicolumn{7}{|c|}{ Au/Pt weight ratio } & Average & Std \\
\hline 1.86 & 1.59 & 1.10 & 0.77 & 0.54 & 0.29 & 0.64 & 1.09 & 0.87 & 0.97 & 0.50 \\
\hline
\end{tabular}



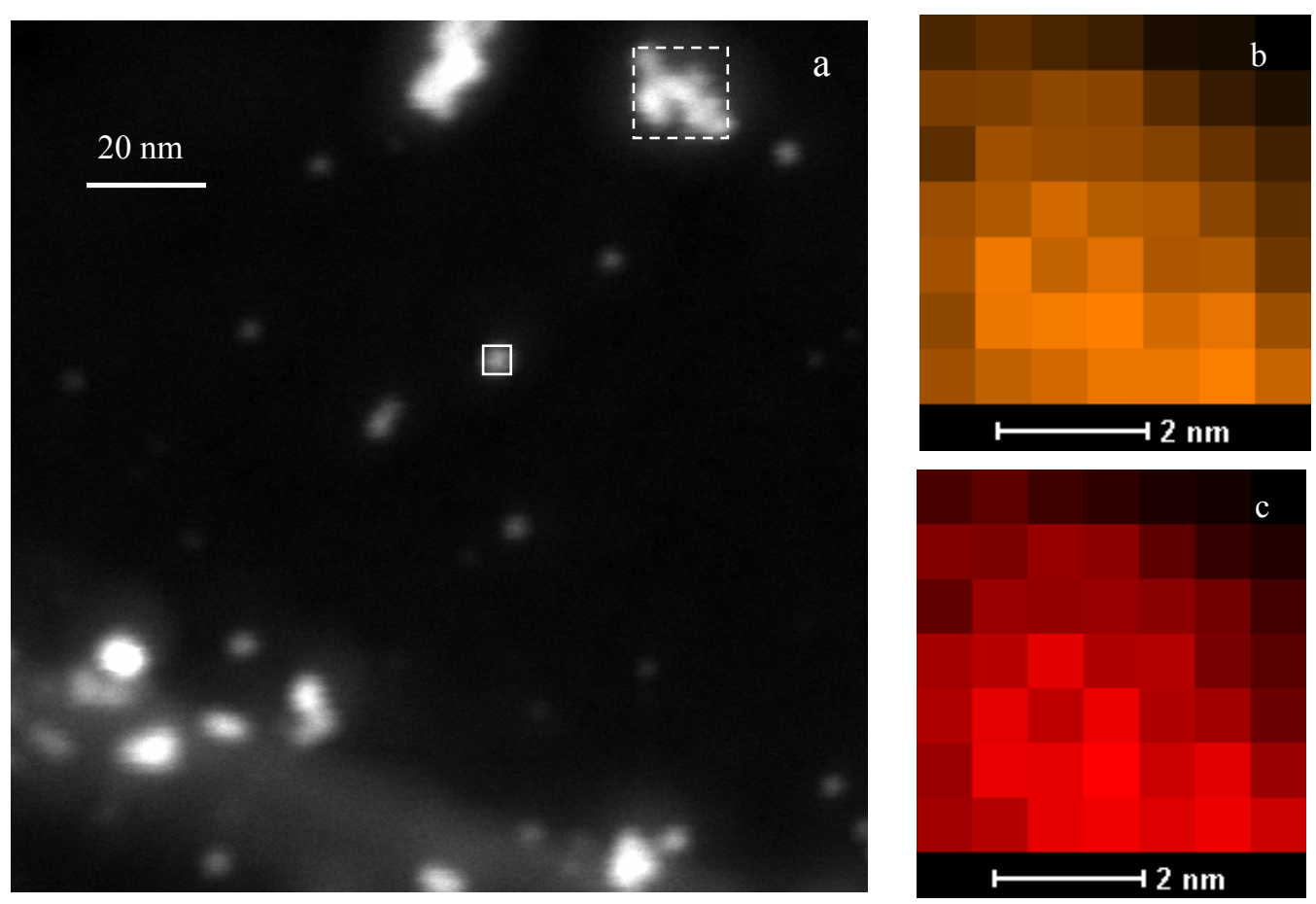

Figure 5.5 EDS compositional mapping of the specified Au-Pt particle $\sim 6 \mathrm{~nm}$ in size (a); Au L (b); Pt L (c)

To investigate the compositional homogeneity within a particle, elemental maps were recorded for a number of single particles using EDS in the STEM mode.

A typical result in the form of a $7 \times 7$ grid on the particle $5.5 \mathrm{~nm}$ in diameter, highlighted by the solid frame in Figure 5.5.a can be seen in Figures 5.5.b and 5.5.c. The drift was checked and corrected for by using the agglomerate in the dash lined frame in Figure 5.5.a as a reference. The compositional mappings based on the Au L edge and the Pt L edge, are shown in Figure 5.5.b and 5.5.c, respectively. The patterns are very similar for the two metals, in particular at the outer rim. This indicates a rather good degree of mixing with no surface segregation. The result is in agreement with a computational study by Xiao et al. (2006) according to which Au-Pt particles not exceeding 7000 atoms (about $6 \mathrm{~nm}$ in diameter) have a negative heat of alloy formation for the full range of concentrations. This can be seen as a surface effect making the alloying of Au and Pt thermodynamically easy in small particles.

Figure 5.6 shows the elemental maps of a significantly larger particle (largest dimension ca. $12 \mathrm{~nm}$, see solid line frame) using a $10 \times 10$ grid. Again the dash lined frame identifies the reference to compensate for drift. It is clear from Figures 5.6.b and 5.6.c that the distributions of $\mathrm{Au}$ and $\mathrm{Pt}$ within the particle are quite alike. The mean concentration of $\mathrm{Pt}$ in the particle is 20 at. \% with a standard deviation of 9.7 at. \%. For the smaller particle $(5.5 \mathrm{~nm})$ mentioned above, the mean Pt concentration is 34 at. \% with a standard deviation of 10 at. \%.

Although the results of Xiao et al. (2006) indicate mixing in full range of concentrations for particle sizes up to $6 \mathrm{~nm}$, our results indicate mixing also for the larger particle in Figure 5.6 We assume that this is because the particle is twinned consisting of smaller units. As the whole sample consists of primary particles hardly larger than $6 \mathrm{~nm}$, we do not expect any demixed cases either for the larger aggregates. 

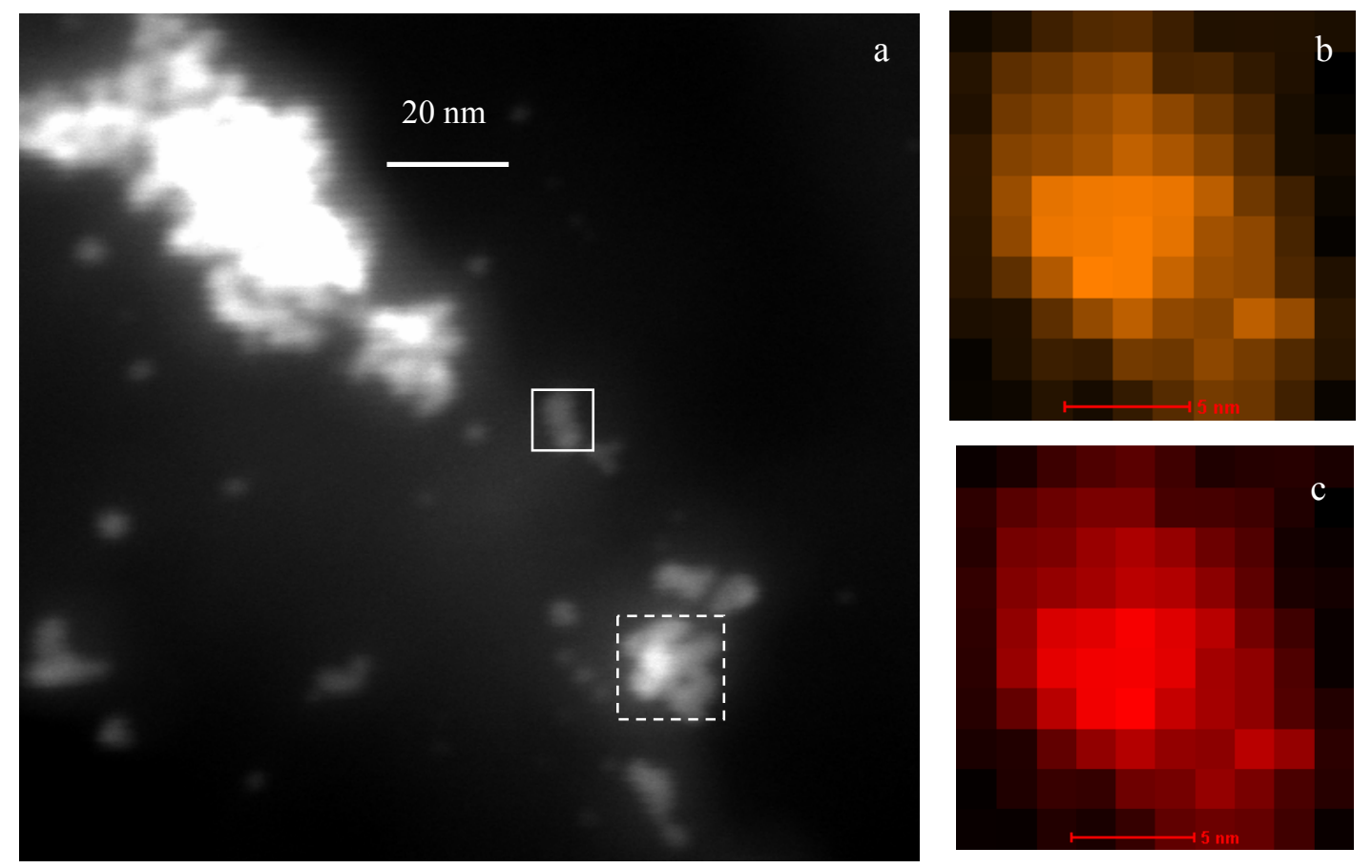

Figure 5.6 EDS compositional mapping of the specified Au-Pt particle (largest dimension ca $12 \mathrm{~nm}$ ) (a); $A u L(b) ; P t L(c)$

The EDS spectrum of a small portion of the particle $(0.7 \mathrm{~nm}$ recorded in $15 \mathrm{~s})$ in Figure 5.7, confirms that both $\mathrm{Au}$ and $\mathrm{Pt}$ are present in comparable concentrations.

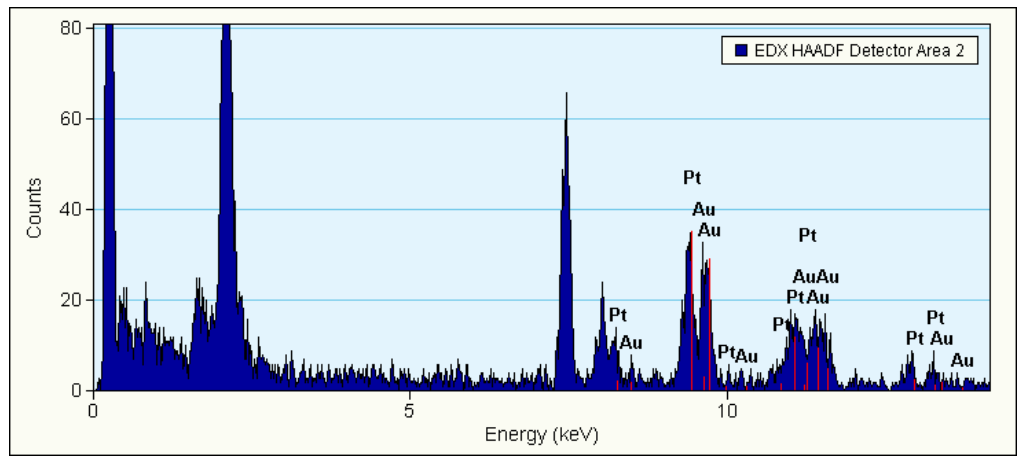

Figure 5.7 A typical EDS spectrum of a small portion of a Au-Pt particle $(\sim 0.7 \mathrm{~nm}$ recorded in $15 \mathrm{~s})$

Average compositions of the particles produced with different electrode polarities were measured by ICP and listed in Table 5.4. It can be concluded that for a specific material, the cathode is more strongly eroded than anode. That is in accordance with our previous results. The fact that we do see substantial erosion of the positive electrode is due to temporary voltage reversal during the discharge process (Tabrizi et al., 2009). The average composition of the nine particles analyzed by EDS (Table 5.3) agrees well with the ICP measurements.

Table 5.4 Compositional analysis of Au-Pt samples measured by ICP

\begin{tabular}{|l|l|}
\hline Electrodes & $\mathrm{Au} / \mathrm{Pt}$ wt. ratio in the sample \\
\hline $\mathrm{Au}(+) \mathrm{Pt}(-)$ & 0.97 \\
\hline $\mathrm{Au}(-) \mathrm{Pt}(+)$ & 1.75 \\
\hline
\end{tabular}


$\mathrm{X}$-ray diffraction patterns of the $\mathrm{Au}(+) \mathrm{Pt}(-)$ and $\mathrm{Au}(-) \mathrm{Pt}(+)$ samples are presented in Figure 5.8. For both arrangements, formation of an intermediate phase is confirmed by Bragg reflections positioned between the peaks of pure gold and pure platinum which is in agreement with the EDS results. The inset shows a close-up of the 220 plane reflections on the $d$ axis scale which directly allows an estimate of the composition of the intermediate phase.

$\mathrm{Au}$ and Pt both have fcc structures with lattice constants of 0.40782 and $0.39242 \mathrm{~nm}$ respectively. Vegard's law predicts a simple linear relationship between alloy composition and lattice spacing for metals with the same crystal structures and atomic sizes (Barret et al., 1996). For $\mathrm{Au}(-) \mathrm{Pt}(+)$ and $\mathrm{Au}(+) \mathrm{Pt}(-)$ arrangements, the alloy phases were approximated to contain around 75 at $\%$ and 60 at $\% \mathrm{Au}$, respectively.

According to the bulk phase diagram of Au-Pt, these compositions fall well in the miscibility gap below the temperatures of $1100 \mathrm{~K}$ and $1400 \mathrm{~K}$ (see Appendix E). Our results are in accordance with Luo et al. (2005), who reported that their bimetallic nanoparticles, synthesized by wet chemistry, displayed alloy properties in contrast to the miscibility gap known for the bulk phase diagram. A slight amount of pure metals was also detected in the samples (see inset). Here the peak sharpness indicates a large particle size, probably due to solidified droplets ejected from the electrode surface, as these have been seen before in the case of Ag above.

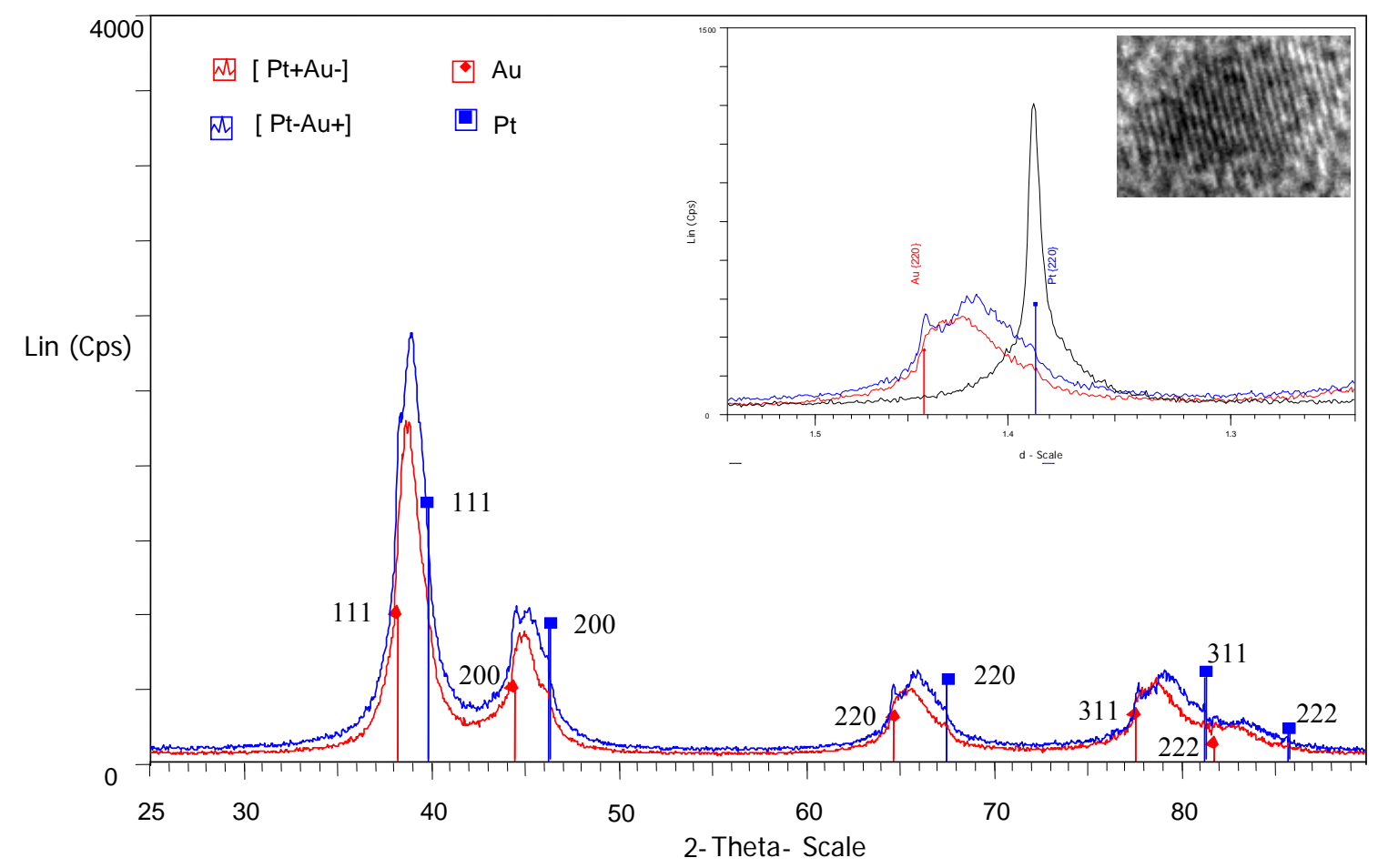

Figure 5.8 X-ray diffraction patterns of Au-Pt sample

Table 5.5 lists the BET surface areas of $\mathrm{Au}$, Pt and Au-Pt nanoparticles. Au and Pt both showed specific surface areas corresponding to BET diameters much larger than typical primary particle diameters. In the case of $\mathrm{Pt}-\mathrm{Au}$, the BET diameter is also larger than the primary particle size observed in the micrograph in Figure 5.4.c. The Au-Pt particles showed a significantly larger specific surface area than the pure metals, indicating reduced cold sintering between the particles. Reduced sintering of the mixed phase had also been observed in our previous work (Tabrizi et al., 2009) for systems which were miscible in bulk scale. 
Table 5.5 Specific surface area of the Au-Pt particles

\begin{tabular}{|c|c|c|}
\hline Material & $\begin{array}{c}\text { BET specific } \\
\text { surface area }\left(\mathrm{m}^{2} / \mathrm{g}\right)\end{array}$ & $\begin{array}{c}\text { Calculated BET } \\
\text { diameter }(\mathrm{nm})\end{array}$ \\
\hline $\mathrm{Au}-\mathrm{Pt}$ & 32 & $9-10$ \\
\hline $\mathrm{Au}$ & 14 & 20 \\
\hline $\mathrm{Pt}$ & $<10$ & $>28$ \\
\hline
\end{tabular}

\subsubsection{Cu-W}

Copper-tungsten nanoparticles were produced from a pair of sintered electrodes. The TEM image of the particles in Figure 5.9. a shows that the primary particles have a rather broad size distribution. The inset with higher magnification reveals that the particles are covered by a surface layer. Due to enhanced chemical reactivity of nanosized particles, small amounts of impurities in the carrier gas, in particular oxygen may be sufficient for chemical modification of the surface layer, and we assume that an oxide layer has formed. TEM micrographs in Figure 5.9 reveal contrast changes on a very small scale (see the inset). We ascribe the darker contrast to $\mathrm{W}$ (atomic no. 74) and the lighter one to $\mathrm{Cu}$ (atomic no. 29).

The SEM image of the particle assemblies in Figure 9.b, shows that the particles are mainly nano-sized and that a few large round particles are also present.
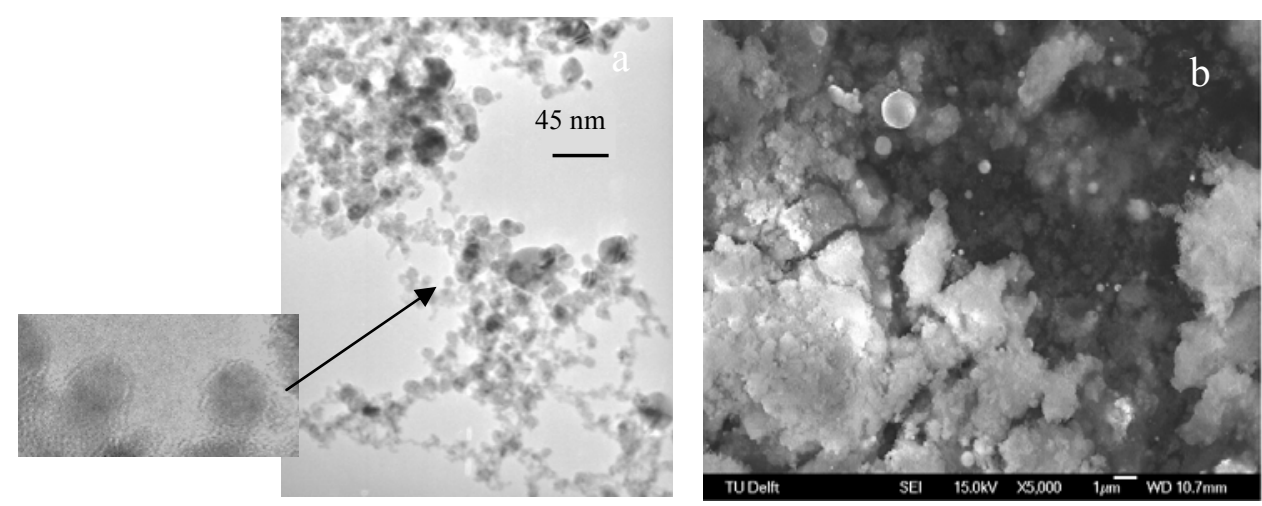

Figure 5.9 TEM image of the $C U / W$ nanoparticles (a), SEM image of the $C u / W$ particles with scale bar representing $1 \mu \mathrm{m}(b)$

The atomic compositions of a number of nanosized particles $(5-12 \mathrm{~nm})$ were determined by EDS. Table 5.6 shows the composition of 10 particles each of them containing both $\mathrm{Cu}$ and $\mathrm{W}$. The average atomic composition agrees with the elemental composition of the electrodes (weight ratio: W72/Cu28 or atomic ratio: W47/Cu53). In addition, fixed point EDS analysis with a beam profile smaller than $0.3 \mathrm{~nm}$, was performed on each particle at six points. The drift correction was performed by comparing to highlighted regions in the solid frames as references. A typical EDS spectrum of a point is shown in Figure 5.10. The EDS line scans on six particles in Figure 5.11 show that the particles are not compositionaly homogeneous.

X-ray photoelectron spectroscopy (XPS) was applied to determine the average elemental composition of the sample surface (Table 5.7). The ratio of the metal compositions agrees well with the composition of the sintered electrodes. 


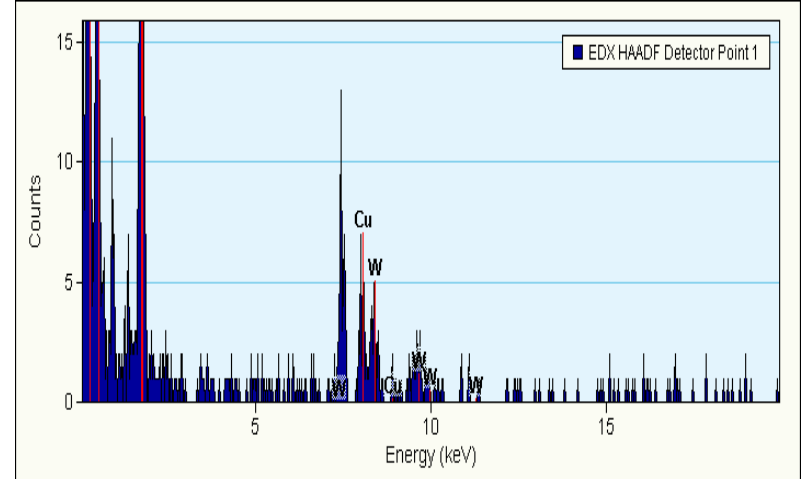

Figure 5.10 A typical EDS spectrum recorded from a small portion of a $\mathrm{Cu}$-W particle ( $\sim 0.7 \mathrm{~nm}$ recorded in $15 \mathrm{~s})$

\begin{tabular}{|l|r|r|}
\hline \multicolumn{1}{|l}{ Cu } & W \\
\hline & 64.298 & 35.701 \\
\hline & 63.543 & 36.456 \\
\hline & 0.527 & 99.472 \\
\hline & 44.565 & 55.434 \\
\hline & 76.221 & 23.778 \\
\hline & 28.356 & 71.643 \\
\hline & 59.172 & 40.827 \\
\hline & 56.989 & 43.01 \\
\hline & 41.655 & 58.344 \\
\hline & 43.527 & 56.472 \\
\hline ave & 47.8853 & 52.1137 \\
\hline & & \\
\hline
\end{tabular}

Table 5.6 Typical atomic compositions of the $\mathrm{Cu}$-W particles

The XPS results also show a remarkable amount of oxygen and carbon. The spectra reveal the presence of $\mathrm{CuO}, \mathrm{Cu}(\mathrm{OH})_{2}, \mathrm{WO}_{2}$, and $\mathrm{WO}_{3}$, which is explained by the fact that exposure of the sample to air could not be avoided before analysis.

Table 5.7 Compositional analysis of $C u$-W sample measured by XPS

\begin{tabular}{|l|l|l|}
\hline Material & $\mathrm{Cu} / \mathrm{W}$ wt. ratio in the electrode & $\mathrm{Cu} / \mathrm{W}$ wt. ratio on the surface of the sample \\
\hline $\mathrm{Cu}-\mathrm{W}$ sintered electrodes & 0.388 & 0.396 \\
\hline
\end{tabular}
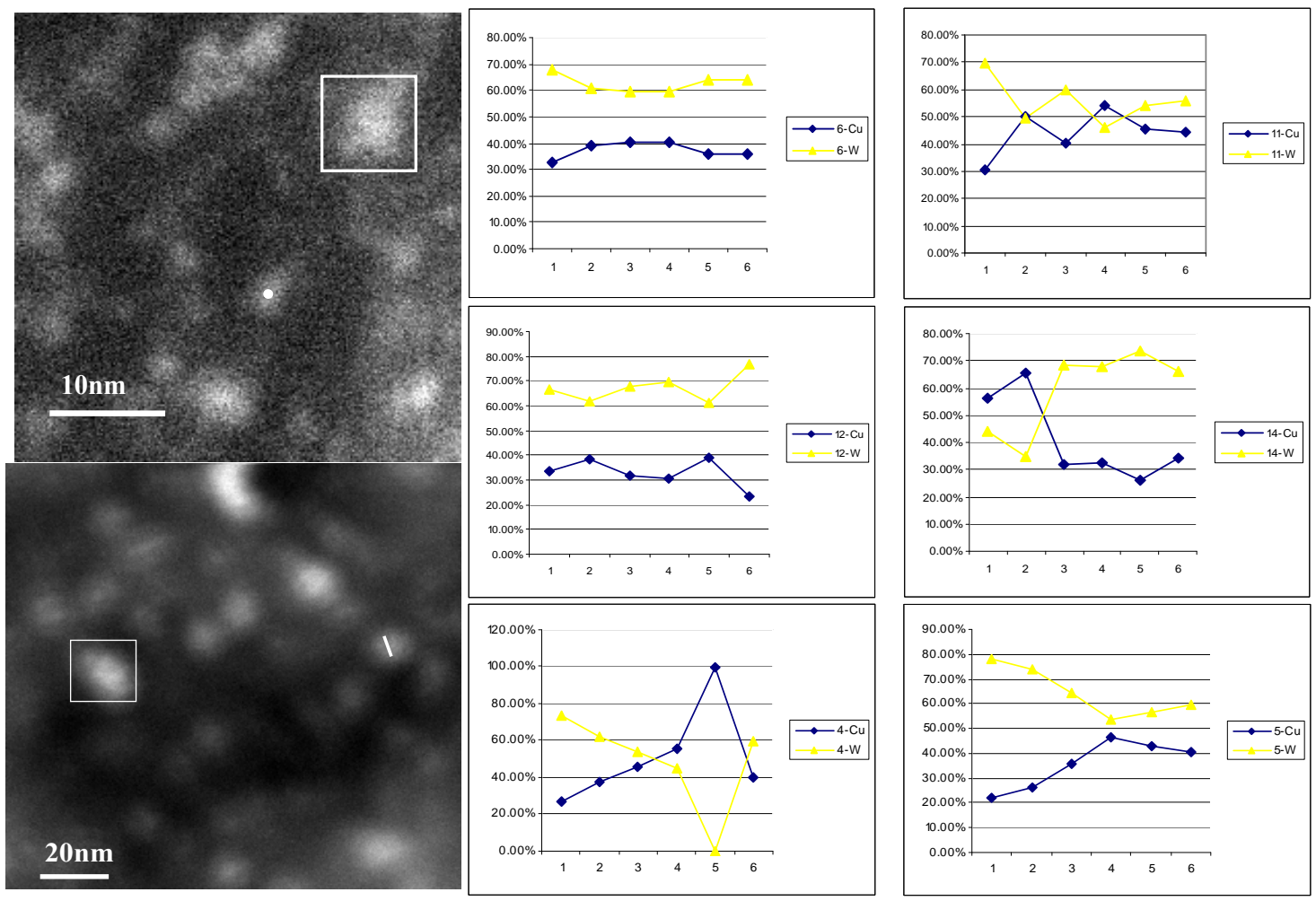

Figure 5.11 Electron micrographs of the Cu-W particles with EDS line scans 
With a large positive heat of mixing $\left(\Delta \mathrm{H}_{f}=+36 \mathrm{~kJ} / \mathrm{mol}\right), \mathrm{Cu}$ and $\mathrm{W}$ are immiscible even in the liquid state (Gladyszewski et al., 1993; Ouyang et al., 2006; Haubold, 1992). Cu and W have fcc and bcc (body-centered cubic) structures with atomic radii of $0.1278 \mathrm{~nm}$ and 0.137 $\mathrm{nm}$, respectively. If $\mathrm{W}$ dissolves in the $\mathrm{Cu}$ lattice, the lattice parameter of $\mathrm{Cu}$ should increase and similarly a decrease in lattice parameter is expected for $\mathrm{W}$ if $\mathrm{Cu}$ dissolves in the $\mathrm{W}$ lattice (Raghu et al., 2001).

Equilibrium phases in the phase diagram of bulk $\mathrm{Cu}-\mathrm{W}$ calculated from a thermodynamic model include the fcc terminal solid solution $(\mathrm{Cu})$ with extremely limited solid solubility of $\mathrm{W}$, and the bcc terminal solid solution (W) with extremely limited solid solubility of $\mathrm{Cu}$ (Massalski et al., 1990). On the basis of first principles calculations, Shu et al. (2003) suggested that the noble metal atoms $(\mathrm{Ag}, \mathrm{Au}, \mathrm{Cu})$ would like to occupy the vacancy sites of the W(001) surface to form the substitutional surface alloys despite the fact that they hardly form an alloy in the bulk. Dirks et al. (1985) produced $\mathrm{Cu}-\mathrm{W}$ alloy films by simultaneous vapour deposition of the elements on unheated substrates.

They found that at least 10 at.\% W may be dissolved in fcc $\mathrm{Cu}$ films and 40 at.\% $\mathrm{Cu}$ may be accommodated in the bcc $\mathrm{W}$ alloy films. They concluded that the vapour quenching technique could lead to the formation of homogeneous one-phase alloys over a wide range of compositions. They estimated that the metastable two phase $(\mathrm{fcc}+\mathrm{bcc})$ coexistence region should lie between 40-60 at.\% tungsten. This is almost in the compositional range of the measured particles in Table 5.6.

Metastable mutual solid solubility in the ball milled $\mathrm{Cu}-\mathrm{W}$ nanocrystallites was also reported by Raghu et al. (2001). According to Xiao et al. (2007), there is a competition between size effect and compositional effect on the heat of formation of immiscible systems. When the formation enthalpy reduces to a smaller value than the interface energy of the system because of the size effects, interface alloying can occur (Liang et al., 2005).

The X-ray diffraction pattern of the sample in Figure 5.12 shows characteristic peaks corresponding to the reflections of the initial constituents as well as $\mathrm{W}_{3} \mathrm{O}$ with $\mathrm{A} 15$ structure. The sharpness of the peaks indicates that they are reflected by the large particles as seen in the SEM image (Figure 5.9.b). It is interesting to note that X-ray diffraction does not show any indication of a nanocrystalline phase, which should manifest itself as broadened lines. On the other hand, the existence of nanoparticles is clearly confirmed by electron microscopy as well as the BET analyses. We conclude that the particles must be in amorphous phase. Indeed, the dominance of smeared continuous rings in the inset of the Figure 5.12 gives further support to an amorphous or quasi-amorphous phase. The electron diffraction pattern in the inset of Figure 5.12 was obtained from an area, where only nanoparticles were present. It exhibits some small diffraction spots arranged along the rings, indicating very small crystallites to be present. Examples of these are shown in Figure 5.13. The calculated $d$ spacing of the framed nanoparticles from the diffraction patterns in Figure 5.13 reveals fcc crystalline structure (Appendix E) with a lattice constant close to copper (FM3-M 225 $\mathrm{a}=0.3613 \mathrm{~nm})$. 


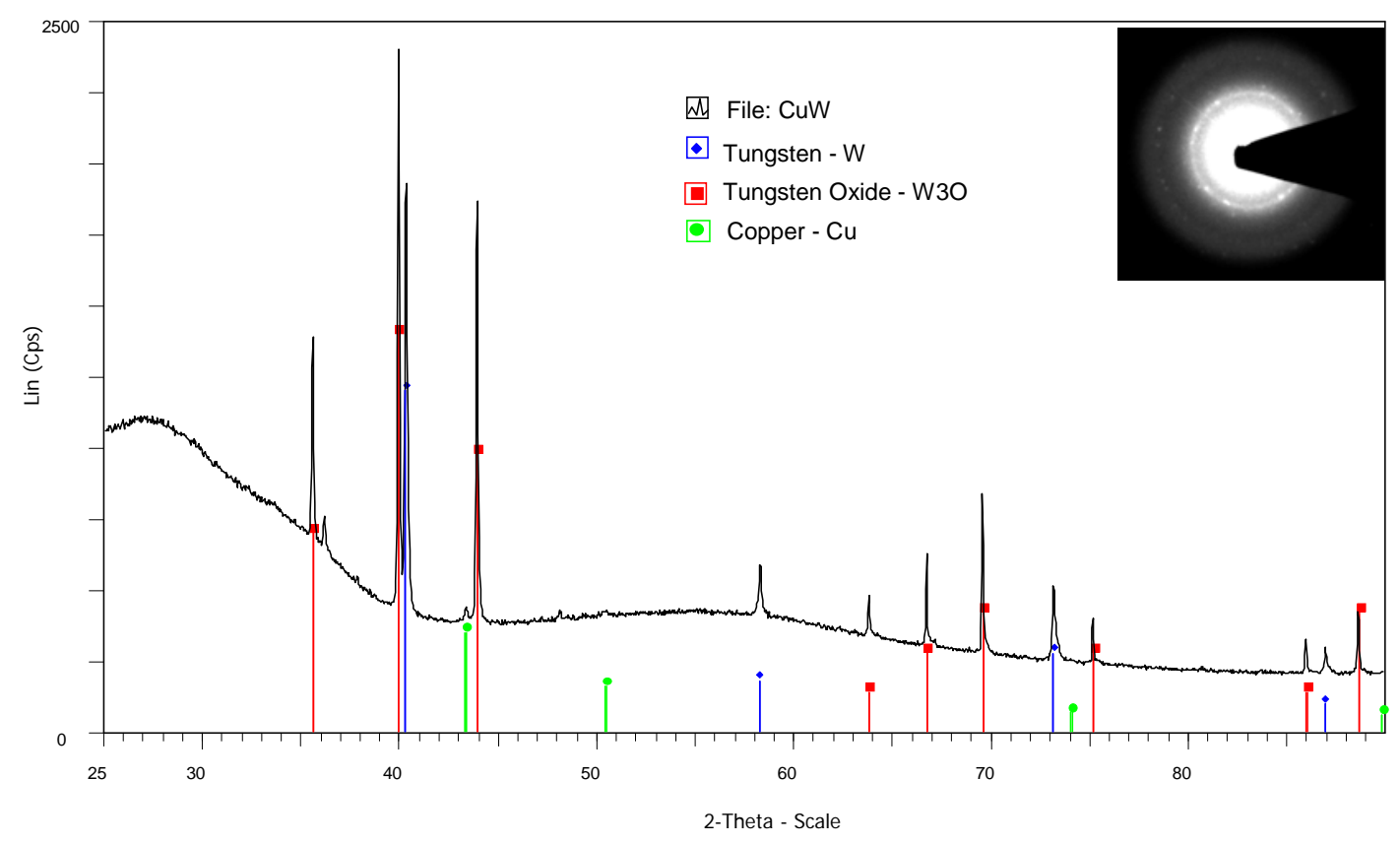

Figure $5.12 \mathrm{X}$-ray diffraction pattern of the $\mathrm{Cu}$-W particles

Together with the EDS line scans of Figure 5.11, we conclude that we have mixed $\mathrm{Cu}$ and W on a scale larger than the lattice constants but significantly smaller than the particle diameters. The EDS line scan on the upper left side of Figure 5.11 would be consistent with a homogeneous mixing, but the line scan on the lower left side shows that very significant changes in composition occur on the scale around one nanometre.

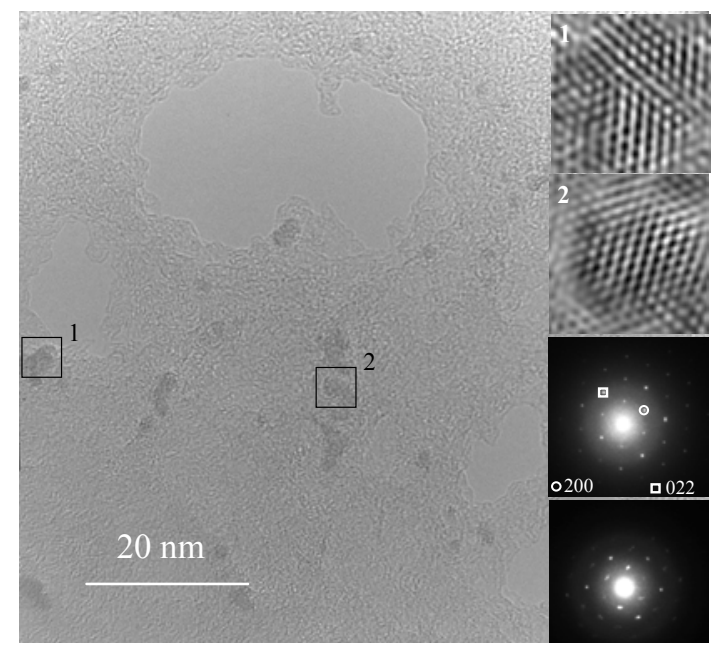

Figure 5.13 Electron diffraction patterns of the specified particles

The specific surface areas of $\mathrm{Cu}, \mathrm{W}$, and $\mathrm{Cu}-\mathrm{W}$ nanoparticles are listed in Table 5.8. The calculated BET diameters are consistent with the mobility particle sizes shown in Figure 5.14 . 
Table 5.8 Specific surface area of the $\mathrm{Cu}$ - $W$ particles

\begin{tabular}{|c|c|c|}
\hline Material & BET specific surface area $\left(\mathrm{m}^{2} / \mathrm{g}\right)$ & Calculated BET diameter $(\mathrm{nm})$ \\
\hline $\mathrm{Cu}-\mathrm{W}$ & 109 & $3-6$ \\
\hline $\mathrm{Cu}$ & 130 & 5.1 \\
\hline $\mathrm{W}$ & 72 & 4.3 \\
\hline
\end{tabular}

In these mobility size distributions, the average $\mathrm{Cu}-\mathrm{W}$ particle size seems to lie between the ones of $\mathrm{Cu}$ and $\mathrm{W}$. The presence of $\mathrm{W}$ reduces the amount of vapor produced per spark with respect to $\mathrm{Cu}$, in agreement with the findings of chapter 2 . As also shown in chapter 2, the particle size decreases with a smaller amount of vapor.

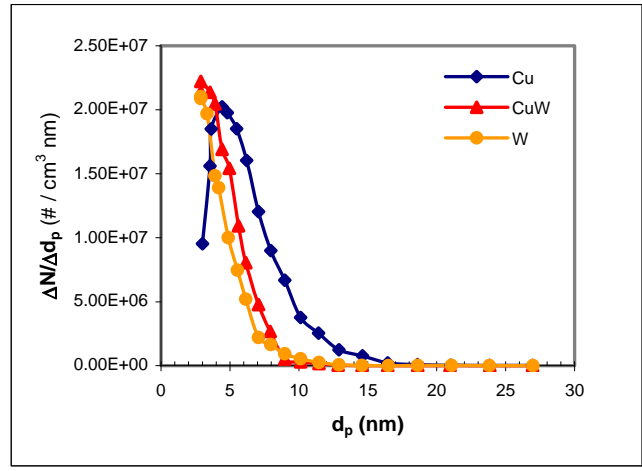

Figure 5.14 Size distributions of $\mathrm{Cu}, W$ and $\mathrm{Cu}$-W nanoparticles $(C=5 \mathrm{nF}, d=1 \mathrm{~mm}, \mathrm{f}=10 \mathrm{~Hz}, Q=5 \mathrm{lpm} \mathrm{Ar})$

\subsection{Conclusion}

Using sintered electrodes of $\mathrm{Ag} / \mathrm{Cu}$ in spark discharge generator, we produced nanoparticles which exhibited enhanced solid solubility of $\mathrm{Cu}$ in $\mathrm{Ag}$ and $\mathrm{Ag}$ in $\mathrm{Cu}$ with respect to the macroscopic case. Effective mixing is also possible by using two electrodes of different compositions, as the circuit applied leads to field reversal during the discharge process. Au-Pt particles produced this way, show intermediate phases with compositions which lie in miscibility gap in bulk phase diagram at ambient temperatures. In agreement with this, EDS elemental mapping of a $6 \mathrm{~nm}$ particle revealed good mixing. This is in agreement with computation by Xiao et al. (2006). Since gold and platinum are both noble metals with low chemical reactivity, cleanness of the particle surfaces leads to cold sintering and consequently low specific surface areas for pure particles. Admixing of $\mathrm{Au}$ and $\mathrm{Pt}$, results in an increase in specific surface area of the mixed particles. Applying sintered electrodes of $\mathrm{Cu} / \mathrm{W}$, we produced mixed particles a few nanometres in size containing both $\mathrm{Cu}$ and $\mathrm{W}$. EDS line scans together with TEM, XRD and electron diffraction give evidence of mixing on a subnanometre scale but above the scale of atomic mixing. In conclusion, spark discharge ablation is a powerful technique for producing new mixed nanoparticulate phases that do not exist in macroscopic systems. Besides using the method for basic studies, the possibility of scaling it up by using multiple discharges bears the potential of producing new materials on a larger scale. 


\section{References}

Almtoft K. Pagh, A. M. Ejsing, J. Bottiger, J. Chevallier, the Dependence of the Nanostructure of Magnetron Sputtered Cu-Ag Alloy Films on Composition and Temperature, Journal of Materials Research, Volume 22, Number 4, 2007, pp. 1018-1023

Barret C. S., et al, the Structure of the Metals, McGraw-Hill, New York, 1996, pp. 372

Birringer R., Nanocrystalline Materials, Material Science and Engineering, Volume A117, 1989, pp. 33-43

Cagran C., B. Wilthan, G. Pottlacher, Enthalpy, Heat of Fusion and Specific Electrical Resistivity of Pure Silver, Pure Copper and the Binary Ag-28Cu Alloy, Thermochimica Acta, Volume 445, 2006, pp. 104-110

Ceylan A., K. Jastrzembski, S. Ismat Shah, Enhanced Solubility Ag-Cu Nanoparticles and their Thermal Transport Properties, Metallurgical and Materials Transactions A, Volume 37A, 2006, pp. 2033-2038

Chatterjee A., D. Chakravorty, Electrical Conductivity of Sol-Gel Derived Metal Nanoparticles, Journal of Materials Science, Volume 27, 1992, pp. 4115-4119

Christensen A., P. Stoltze , J. K. Norskov, Size Dependence of Phase Separation in Small Bimetallic Clusters, Journal of Physics:Condensed Matter, Volume 7, 1995, pp. 1047-1057

Devarajan Supriya, Parthasarathi Bera, S. Sampath, Bimetallic Nanoparticles: A Single Step Synthesis, Stabilization, and Characterization of $\mathrm{Au}-\mathrm{Ag}, \mathrm{Au}-\mathrm{Pd}$, Au-Pt, in Sol-Gel Derived Silicates, Journal of Colloid and Interface Science, Volume 290, 2005, pp. 117-129

Ding Feng, Arne Rosen, Kim Bolton, Size Dependence of the Coalescence and Melting of Iron Clusters: A Molecular-Dynamics Study, Physical Review B, Volume 70, 2004, pp. 075416

Dirks A. G., J. J. van den Broek, Metastable Solid Solution in Vapour Depositd Cu-Cr, Cu-Mo, and Cu-W Thin Films, J. Vac. Sci. Technol. A, Volume 3, Number 6, 1985, pp. 2618-2622

Gladyszewski G., Ph. Goudeau, A. Naudon, C. Jaouen, J. Pacaud, Modification of Cu-W Superlattices by Irradiation, Applied Surface Science, Volume 65/66, 1993, pp. 28-3

Hajra J. P., S. Acharya, Thermodynamics and Phase Equilibria Involving Nano Phases in the Cu-Ag System, Journal of Nanosicence and Nanotechnology, Volume 4, Number 7, 2004, pp. 899-906

Haubold T., V. Gertsman, on the Structure and Properties of Nanostructured Copper-Tungsten Alloys, Nanostructured Materials, Volume 1, 1992, pp. 303-312

Jiang Hongjing, Kyoung-sik Moon, C. P. Wong, Synthesis of Ag-Cu Alloy Nanoparticles for Lead-Free Interconnect Materials, IEEE, 2005

Kang Hyun-Ki, Suk Bong Kang, Tungsten/Copper Composite Deposits Produced by a Cold Spray, Scripta Materialia, Volume 49, 2003, pp. 1169-1174

Koch Carl C., Nanostructured Materials, William Andrew, 2007

Kubaschewski O., C. B. Alcock, Metallurgical Thermo-Chemistry, International Series on Materials Science and Technology, Volume 24, Pergamon International Library, 1979

Lahiri Debdutta, Bruce Bunker, Bhoopesh Mishra, Bimetallic Pt-Ag and Pd-Ag Nanoparticles, Journal of Applied Physics, Volume 97, 2005, pp. 094304-1

Liang L. H., G. W. Yang, Baowen Li, Size- Dependent Formation Enthalpy of Nanocompounds, J. Phys. Chem. $B$, Volume 109, 2005, pp. 16081-16083 
Luo Jin, Mathew M. Maye, Valeri Petkov, Nancy N. Kariuki, Lingyan Wang, Peter Njoki, Derrick Mott, Yan Lin, Chuan-Jian Zhong, Phase Properties of Carbon-Supported Gold-Platinum Nanoparticles with Different Bimetallic Compositions, Chemistry of Materials, Volume 17, 2005, pp. 3096-3091

Massalski, Thaddeus B., Okamito, Hiroaki, Binary Alloy Phase Diagrams, Volume 2, Metals Park, ASM International, 1990

Mezey L. Z., J. Giber, the Surface Free Energies of Solid Chemical Elements: Calculation from Internal Free Enthalpies of Atomization, Japanese Journal of Applied Physics, Volume 21, Number 11, 1982, pp. 1569-1571

MPR, June, 2000, 8

Najafabadi R., D. J. Srolovitz, Thermodynamic Properties of Metastable Ag-Cu Alloys, Journal of Applied Physics, Volume 74, Number 5, 1993, pp. 3144-3149

Ouyang G., C. X. Wang, S. W. Li, X. Zhou, G. W. Yang, Size-Dependent Thermodynamic Criterion for the Thermal Stability of Binary Immiscible Metallic Multilayers, Applied Surface Science, Volume 252 , Number 11, 2006, pp. 3993-3996

Ouyang G., X. Tan, C. X. Wang, G. W. Yang, Charge-Induced Transition between Miscible and Immiscible in Nanometer-Sized Alloying Particles, Chemical Physics Letters, Volume 423, 2006, pp. 143-146

Patel Kirti, Sudhir Kapoor, Devilal Purshottam Dave, Tulsi Mukherjee, Synthesis of Pt, Pd, Pt/Ag and Pd/Ag Nanoparticles by Microwave-Polyol Method, Journal of Chemical Sciences, Volume 117, Number 4, 2005, pp. 311-316

Powell A., J. Van Den Avyle, B. Damkroger, J. Szekely and U. Pal, Analysis of Multicomponent Evaporation in Electron Beam Melting and Refining of Titanium Alloy, Metall. Mater. Trans., volume 28B, Number 6, 1997 , pp. $1227-1239$

Predel B., O. Madelung, Phase Equilibria, Crystallographic and Thermodynamic Data of Binary Alloys, Belrin, Springer, 1998

Radic N., M. Stubicar, Microhardness Properties of $\mathrm{Cu}-\mathrm{W}$ Amorphous Thin Films, Journal of Materials Science, Volume 33, 1998, pp. 3401-3405

Raghu T., R. Sundaresan, P. Ramakrishnan, T. R. Rama Mohan, Synthesis of Nanocrystalline Copper-Tungsten Alloys by Mechanical Alloying, Material Science and Engineering, Volume A 304-306, 2001, pp. 438-441

Rouquerol Francoise, Jean Rouquerol, Kenneth Sing, Adsorption by Powders and Porous Solids: Principles, Methodology and Applications, San Diego Academic Press, 1999

Shu X. K., P. Jiang, J. G. Che, Surface Alloying of Immiscible Metals Induced by Surface State Shift, Surface Science, Volume 545, 2003, pp. 199-210

Somasundaran Ponisseril, Encyclopedia of Surface and Colloid Science, Taylor \& Francis, 2006

Tabrizi N. S., M. Ullmann, V. A. Vons, U. Lafont, A. Schmidt-Ott, Generation of Nanoparticles by Spark Discharge, Journal of Nanoparticle Research, Volume 11, 2009, pp.315-332

Tabrizi N. S., Q. Xu, N. M. Van der Pers, A. Schmidt-Ott, Generation of Mixed Metallic Nanoparticles from Immiscible Metals by Spark Discharge, Journal of Nanoparticle Research, DOI: 10.1007/s11051-009-9603-4

Wu Ming-Li, Long-Biao Lai, Synthesis of Pt/Ag Bimetallic Nanoparticles in Water-in-oil Microemultions, Colloids and Surfaces A: Physicochem. Eng. Aspects, Volume 244, 2004, pp. 149-157

Xiao S., W. Hu, W. Luo, Y. Wu, X. Li, H. Deng, Size Effect on Alloying Ability and Phase Stability of Immiscible Bimetallic Nanoparticles, The European Physical Journal B, Volume 54, 2006, pp. 479-484 
Zeng Jianhuang, Jun Yang, Jim Yang Lee, Weijiang Zhou, Preparation of Carbon-Supported Core-Shell Au-Pt Nanoparticles for Methanol Oxidation Reaction: The Promotional Effect of the Au Core, J. Phys. Chem. B, Volume 110, 2006, pp. 24606-24611 


\section{Chapter 6}

\section{Theoretical Prediction of primary Particle Size}

\subsection{Introduction}

Prediction and control of primary particle size of nanostructured materials is of great importance since it is a key parameter to tailor the size dependent properties of the material for a specific application.

Synthesis of metallic nanoparticles in the gas phase consists basically of two steps: formation of the vapor, and subsequent cooling to condense the metal vapor molecules. The collisions of the gas atoms with the metal vapor cool the metal atoms and reduce the diffusion rate while inducing supersaturation. Homogeneous nucleation, or self-nucleation, whereby the clusters themselves serve as nucleation sites, occurs only under a substantial supersaturation.

After nucleation, particles usually continue to grow by acquiring more atoms from the vapor. In addition, growth occurs when the particles collide with each other and then coalesce to form larger units. Growth at high particle concentration is governed by the collision and coalescence rates. If coalescence is faster (for example at high temperatures), large spherical particles with a small surface area, form. If the collision rate is faster (for example at low temperatures), dendritic agglomerates consisting of a large number of small primary particles with large surface area are produced, see Figure 6.1.

Upon further heating, the agglomerated particles fuse into more compact structures. Depending on the strengths of bonding between the primary particles, different morphologies may be formed. Strong bondings (for example ionic bonds) may lead to growth of primary particles through heating, but with weak bondings the agglomerates restructure to form closepacked aggregates (Schmidt-Ott, 1988; Weber and Friedlander, 1997).

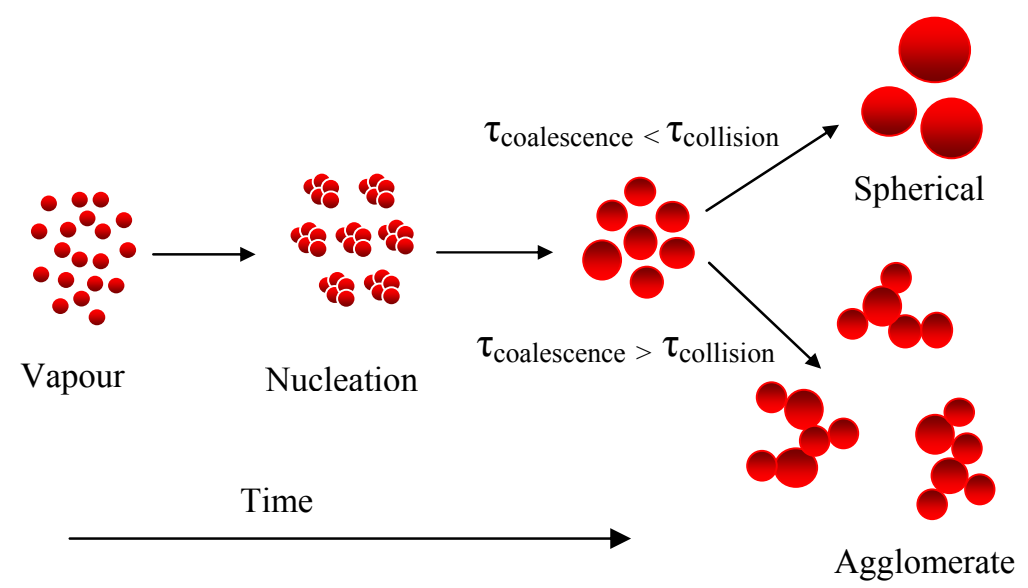

Figure 6.1 Gas phase particle growth (reproduced from Zachariah and Carrier, 1999)

Controlling the coalescence rate is possible through knowledge of the material properties and the time-temperature history of the reactor, and the collision rate can be controlled through the volume loading of the material (Lehtinen and Zachariah, 2001). 
Molecular Dynamic simulation (Zachariah and Carrier, 1999) showed that when two particles coalesce, the formation of new chemical bonds and reduction of surface area and surface free energy result in energy release and hence a significant increase in particle temperature, see Figure 6.2. The study on the kinetics of particle growth (up to $3 \mathrm{~nm}$ ) showed that solid-like particles sinter by solid state diffusion while liquid-like particles sinter by viscous flow.

Coalescence by solid state diffusion has a rate which is an exponential function of temperature. Thus the temperature increase within the particle has an important effect on the dynamics of coalescence. Here heat transfer to the gas also plays an important role.

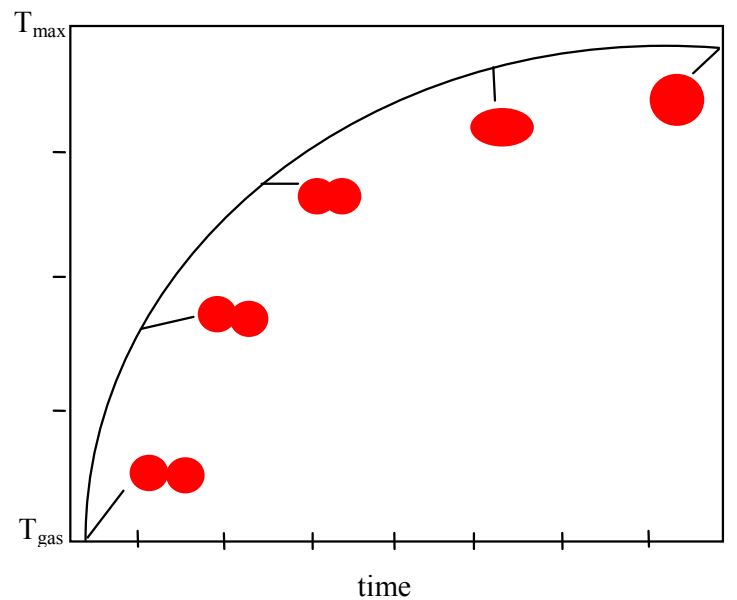

Figure 6.2 Coalescence in nanoparticles showing a temperature increase due to energy release as a result of decreasing surface area (adapted from Lehtinen and Zachariah, 2002)

Smaller particle sizes are favored by high cooling rates (Panda and Pratsinis, 1995; Singh et al., 2002). Lehtinen and Zachariah (2002) reported that high volume loadings and /or low pressure favor the growth of large primary particles. However other researchers (Panda and Pratsinis, 1995; Simchi et al., 2005) have observed that an increase in the inert-gas pressure leads to an increase in particle size. It is probably the partial vapor pressure of the precursor rather than the overall system pressure which affects the particle size. The total pressure serves to regulate the diffusion of vapor from the growth source. Diffusion increases when the total pressure is lowered so a wide dispersion of the particles restricts the growth by coagulation. An increase in the inert-gas pressure slows the diffusion of metal vapor away from the source so that the vapor is enriched. On the other hand, high pressures shorten the mean free path of atoms and effectively increase the quenching rate, which leads to enhanced nucleation. If the pressure is reduced, the vapor diffuses away from the source faster and nucleation zone becomes more spread out. Depletion of the vapor prevents clusters from growing very large (Koch, 2007).

The choice of the inert gas along with the pressure and temperature gradient are important parameters to control the diffusion rate. Heavier gas atoms are most effective in limiting the mean free path and confining the metal vapor. That is why helium, having a low mass, allows more metal to be removed from the growth region, resulting in smaller particle sizes (Koch, 2007).

Increasing the inert-gas flow rate reduces the length of time that the clusters spend in the growth region of higher vapor density and consequently often leads to a decrease in the particle sizes. It also increases the effectiveness of cooling (Koch, 2007).

High evaporation rates result in larger particles. In addition, the primary particle growth is found (Seipenbusch et al., 2003) to be accelerated strongly with increasing purity of the particle surfaces. 
In coalescence of clusters, the effect of surface curvature is important. The smaller the clusters the larger the surface curvature and the larger the diffusion rates of surface atoms. Since surface diffusion is dominant in coalescence, it can occur without the clusters being molten (Ding et al., 2004).

For unequal sized nanoparticles, MD simulation (Hawa and Zachariah, 2006) showed that the coalescence processes become faster when the ratio of two particle sizes (smaller/larger) approaches zero and the coalescence time of two unequal sized nanoparticles can be predicted by the Koch-Friedlander model.

Koch and Freidlander (1990) generalized the Smoluchowski equation to incorporate the coalescence rate into the aerosol dynamic. According to them the final particle structure depends on three characteristic time scales: the characteristic collision time, the residence time, and the characteristic fusion time. The evolution of the surface area between two limits, the maximum area induced by nonagglomerated particles and the minimum area corresponding to perfectly coalescing particles, depends on the ratio of the characteristic coalescence and collision times.

For predicting primary particle growth, Lehtinen et al. (1996) proposed a model based on the linear decay law for the surface area of a coalescing structure. In the collision limited primary particle growth regime, the linear decay law was applied to the coalescing pairs of particles. In the coalescence-limited regime, where large aggregates were present, the linear decay law was applied to subregions of the agglomerates.

\subsection{Mathematical Model}

Combining the general dynamic equation for the continuous size distribution function, in which the particle number concentration per unit mass of gas is a function of particle volume, surface, and time, with the coalescence rate derived by Koch and Friedlander (1990), the following equation for the fractional deviation of the aerosol surface area from the state in which each particle has relaxed to spherical shape $(\theta)$ is obtained by Lehtinen et al. (1996):

$\frac{d \theta}{d t}+\left[\frac{1}{\tau_{f}(\bar{v})}-\frac{1}{\tau_{c}(\bar{v})}\right] \theta=\frac{1}{\tau_{c}(\bar{v})}$

The size distribution is assumed to remain (nearly) self-preserving for which the rate of decrease in the number of particles per unit mass of gas is given by Flagan and Friedlander (1978). Using this equation together with the average particle volume $\left(\bar{v}=\Phi_{m} / N_{m}\right)$ and aerosol particle volume fraction $\left(\Phi=\rho_{g} \Phi_{m}\right)$ gives an equation for the change of average particle volume with time (for details see Lehtinen et al., 1996):

$\frac{d \bar{v}}{d t}=\frac{1}{2} \alpha\left(\frac{6 k T}{\rho_{p}}\right)^{1 / 2}\left(\frac{3}{4 \pi}\right)^{1 / 6} \Phi \bar{v}^{1 / 6}$

for solving equations (6.1) and (6.2), the following initial conditions are applied:

$\theta(0)=0$

$\bar{v}(0)=v_{0}$ 
where $\theta$ is dimensionless excess surface area compared to spherical particles $\left(\theta=\frac{A_{m}-A_{s p h}}{A_{s p h}}\right)$, $\bar{v}$ average particle volume, $\mathrm{v}_{0}$ initial average particle volume, $\mathrm{k}$ Boltzmann's constant, $\mathrm{N}_{\mathrm{m}}$ number of particles per unit mass of gas, $\mathrm{T}$ temperature, $\mathrm{t}$ time, $\alpha$ constant from selfpreserving theory which is around 6.6 for spherical particles (Wu and Friedlander, 1993), $\rho_{g}$ density of gas, $\rho_{p}$ density of particles, $\Phi$ volume fraction of particles, $\Phi_{m}$ volume of particles per unit mass of gas.

There is a simple relationship between the primary particle diameter $d_{p}, \theta$ and average volume $\bar{v}$, as (Lehtinen et al., 1996):

$d_{p}=\left(\frac{6 \bar{v}}{\pi}\right)^{1 / 3} \frac{1}{\theta+1}$

$\tau_{f}$ and $\tau_{c}$ are coalescence and collision characteristic times respectively (Lehtinen et al., 1996):

$$
\begin{aligned}
& \tau_{f}=\frac{3 k T v}{64 \pi D \sigma v_{m}} \\
& \frac{1}{\tau_{c}}=\frac{1}{3 \bar{v}} \frac{d \bar{v}}{d t}
\end{aligned}
$$

$\mathrm{D}$ is solid state diffusion coefficient, $\sigma$ surface tension, and $\mathrm{v}_{\mathrm{m}}$ molecular volume. Equation (6.7) is valid for monodisperse and self-preserving distributions. Solid state diffusion coefficient is exponentially dependent on the temperature and has an Arrhenius form:

$$
D(T)= \begin{cases}D_{0, v} \exp \left(-\frac{E_{a c t, v}}{k T}\right) & \text { for volume diffusion } \\ D_{0, g b} \frac{b}{d_{p}} \exp \left(-\frac{E_{a c t, g b}}{k T}\right) & \text { for grain boundary diffusion }\end{cases}
$$

where $\mathrm{D}_{0}$ is the prefactor, $\mathrm{b}$ the grain boundary width, and $\mathrm{E}_{\text {act }}$ activation energy.

Thus the primary particle size can be calculated by solving for $\theta$ and $\bar{v}$ as functions of time through equations (6.1) and (6.2) and inserting them in equation (6.5).

Dilution effects can be taken into account through the particle volume fraction $\Phi$ in equation (6.2).

It is noted that the model inputs are material properties, the particle volume fraction $\Phi$, and the time-temperature history of the system (variation of temperature with time which necessitates the knowledge about the initial temperature $\mathrm{T}_{0}$ and the cooling rate $\kappa$ ).

\subsection{Results and Discussion}

Bandyopadhyaya et al. (2003) solved the aerosol dynamics equations (Windeler et al., 1997) to predict the trends of niobia, titania, and alumina particle sizes synthesized by flame reactor 
and laser ablation processes assuming a constant cooling rate $(\kappa)$ and invariant particle volume loading ${ }^{1}(\Phi)$.

In their study primary particle sizes were calculated by equating the coalescence and collision characteristic times determined base on the bulk properties of the oxides listed in Table 6.1 (Windeler et al., 1997).

With the abovementioned assumptions and a linear relationship between temperature and time ( $\left.\mathrm{T}=\mathrm{T}_{0}-\kappa \mathrm{t}\right)$, we solved equations (6.1) and (6.2) numerically with classical fourth-order Runge-Kutta method (Appendix F) ${ }^{2}$ in MATLAB and plugged $\theta$ and $\bar{v}$ into equation (6.5) to estimate the primary particle sizes.

We also computed the coalescence and collision characteristic times (equations 6.6 and 6.7) for these oxides in typical conditions of a flame reactor (Bandyopadhyaya et al., 2003). Our results in Figure 6.3 agree well with the computational results obtained by Bandyopadhyaya et al. (2003).

Table 6.1 Metal oxide properties (Windeler et al., 1997)

\begin{tabular}{|l|l|l|l|}
\hline Property & $\mathbf{N b}_{\mathbf{2}} \mathbf{O}_{\mathbf{5}}$ & $\mathbf{T i O}_{\mathbf{2}}$ & $\mathbf{A l}_{\mathbf{2}} \mathbf{O}_{\mathbf{3}}$ \\
\hline Diffusion pre-exponential, $\mathrm{D}_{0}\left(\mathrm{~m}^{2} \mathrm{~s}^{-1}\right)$ & $1.72 \times 10^{-6}$ & $7.2 \times 10^{-6}$ & 2.5 \\
\hline Diffusion activation energy, $\mathrm{E}_{\mathrm{a}}\left(\mathrm{kJ} \mathrm{mol}^{-1}\right)$ & 206 & 286 & 565 \\
\hline Surface tension, $\sigma\left(\mathrm{N} \mathrm{m}^{-1}\right)$ & 0.75 & 0.6 & 0.905 \\
\hline Vacancy volume, $\mathrm{v}_{0}\left(\mathrm{~m}^{3}\right)$ & $20 \times 10^{-30}$ & $17 \times 10^{-30}$ & $21 \times 10^{-30}$ \\
\hline Density, $\rho\left(\mathrm{kg} \mathrm{m}^{-3}\right)$ & 3960 & 3840 & 4470 \\
\hline Melting temperature $(\mathrm{K})$ & 1653 & 2143 & 2327 \\
\hline Boiling temperature $(\mathrm{K})$ & - & 3245 & 3253 \\
\hline
\end{tabular}

The collision times for all the oxides coincided since it is mainly dependent on the particle volume fraction $(\Phi)$ which was the same in all the cases. However the coalescence time depends strongly on the properties of the material.

According to Windeler et al. (1997), the angle between the collision and coalescence characteristic times at the point of intersection determines the particle shape and extent of necking. At this point, if the coalescence time curve steeply crosses the collision time, spherical particles form otherwise particles are quenched in a state of partial coalescence and necks remain between particles.

It can be seen that the primary particle sizes for all the oxides are fixed at still high temperatures, about $70-80 \%$ of bulk melting temperature (Windeler et al., 1997), corresponding to the intersection point of the characteristic times and in a time scale of few milli-seconds. The model predicts the smallest particle size for alumina with the lowest solid state diffusion coefficient at the given temperature and the largest primary particles for niobia with the largest solid state diffusion. This is in agreement with the TEM analysis results in Table 6.2 obtained by Windeler et al. (1997) from particles produced in a flame reactor. Figure 6.4 shows a typical variation of the particle size with temperature for titania.

\footnotetext{
${ }^{1}$ In general, dilution decreases $\Phi$ resulting in smaller particle sizes. On the other hand, cooling leads to an increase in the particle volume fraction. Thus cooling and dilution have opposite effects on the particle volume loading. Thus, these effects tend to cancel, and $\Phi$ can roughly be regarded as constant (Bandyopadhyaya et al., 2003). The effect of gas type and pressure is reflected in the particle volume fraction $(\Phi)$ through the gas density $\left(\Phi=\rho_{g} \Phi_{m}\right)$.

${ }^{2}$ Analytical solutions (Miller, 1987) are also given in Appendix F.
} 


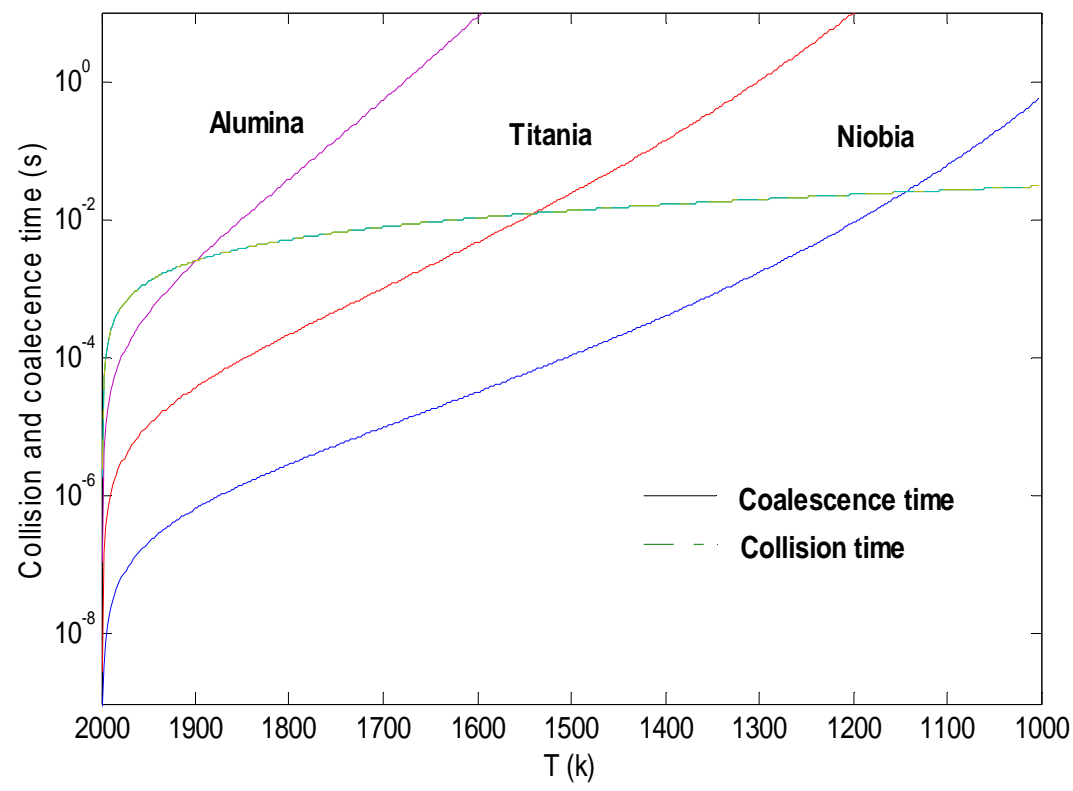

Figure 6.3 Coalescence and collision characteristic times for different oxides in typical operating conditions of a flame reactor $\left(T_{0}=2000 \mathrm{~K}, \kappa=10^{5} \mathrm{Ks}^{-1}, \Phi=10^{-7}\right)$

Table 6.2 Model predictions and experimental data from Flame reactor

\begin{tabular}{|l|l|l|l|}
\hline $\begin{array}{l}\text { Aerosol } \\
\text { material }\end{array}$ & $\mathbf{D}\left(\mathbf{m}^{2} \mathbf{~ s}^{-1}\right)$ & $\begin{array}{l}\text { Geometric mean particle diameter }(\mathbf{n m}) \text {, produced } \\
\text { by Flame reactor (Windeler et al., 1997) }\end{array}$ & $\begin{array}{l}\text { Particle size (nm) } \\
\text { predicted by the model }\end{array}$ \\
\hline Niobia & $8.42 \times 10^{-12}$ & 21.6 & 17.8 \\
\hline Titania & $3.05 \times 10^{-13}$ & 11.1 & 12.3 \\
\hline Alumina & $6.83 \times 10^{-15}$ & 4.1 & 4.6 \\
\hline \multicolumn{3}{|c|}{$\begin{array}{c}\text { Experimental particle volumetric concentration } \Phi=3.2 \times 10^{-7}, \\
\text { (model assumptions: } T_{0}=2030 \mathrm{~K}, \mathrm{\kappa}=1 \times 10^{5} \mathrm{Ks}^{-1} \text { ) }\end{array}$} \\
\hline \multicolumn{2}{|r}{}
\end{tabular}

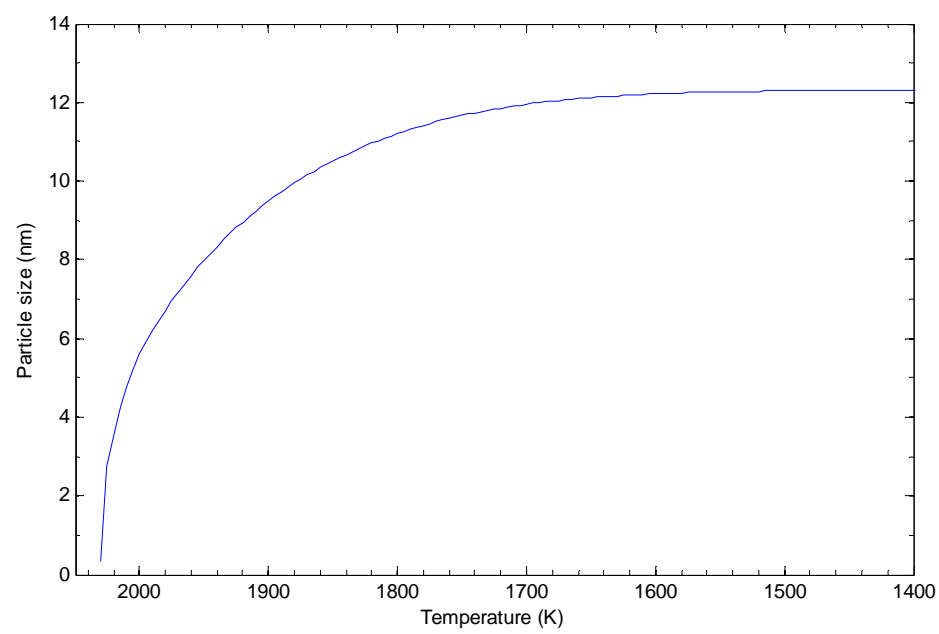

Figure 6.4 Particle size calculation for Titania $\left(T_{0}=2030 \mathrm{~K}, \Phi=3.2 \times 10^{-7}, \kappa=1 \times 10^{5} \mathrm{Ks}^{-1}\right)$ 
Our calculations on the variation of primary particle size with the cooling rate for these oxides are shown in Figure 6.5. It can be seen that particles are larger at lower cooling rates. As the cooling rate increases, the particle sizes decrease and become less material dependent.

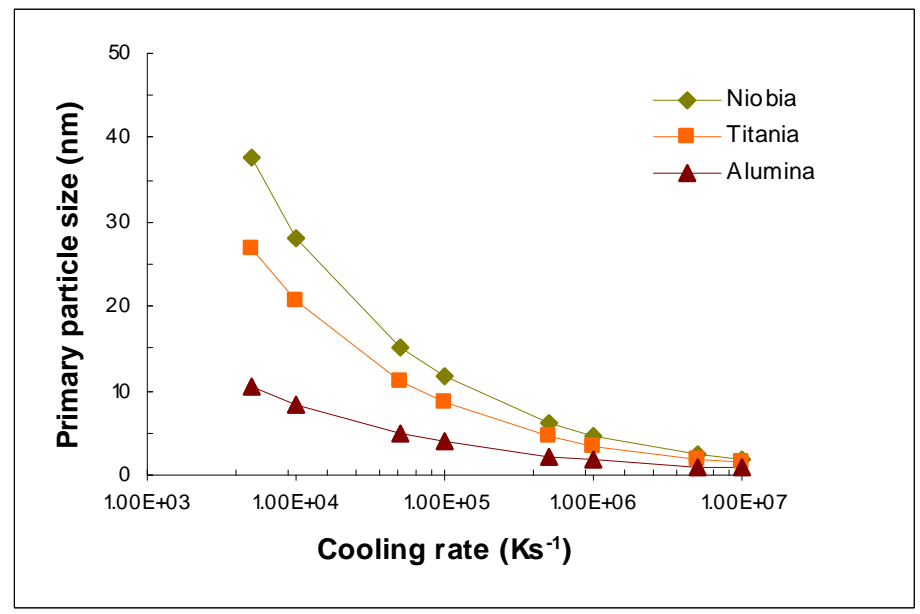

Figure 6.5 Variation of primary particle size of different oxides with cooling rate $\left(T_{0}=2000 \mathrm{~K}, \Phi=10^{-7}\right)$

The relative particle sizes of niobia and alumina (as largest and smallest particles) at various cooling rates are determined from Figure 6.5 and shown in Figure 6.6. It can be seen that as the cooling rate increases the relative particle size decreases.

In spark discharge and laser ablation methods, particles produced from various materials are mainly very small (few $\mathrm{nm}$ ) and in a narrow size range and do not show significant material dependence. This can be seen as a result of the extremely large cooling rate in these methods.

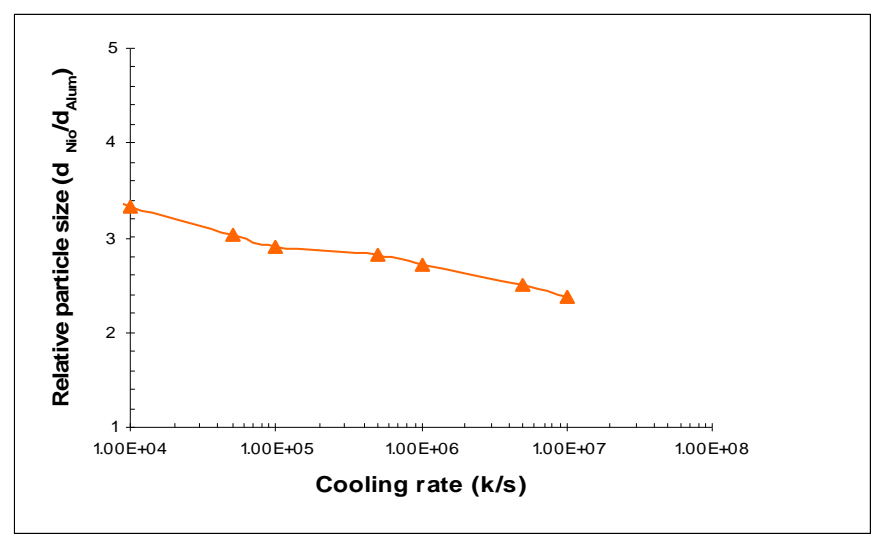

Figure 6.6 Relative particle sizes at different cooling rates

The effect of particle volume loading on the primary particle sizes calculated in this work is presented in Figure 6.7. The results show that the particle sizes increase with the volume loading and the effect is more pronounced for niobia which has the largest solid state diffusion. At low particle volume loadings, the particle sizes for different oxides exhibit a small variation. 


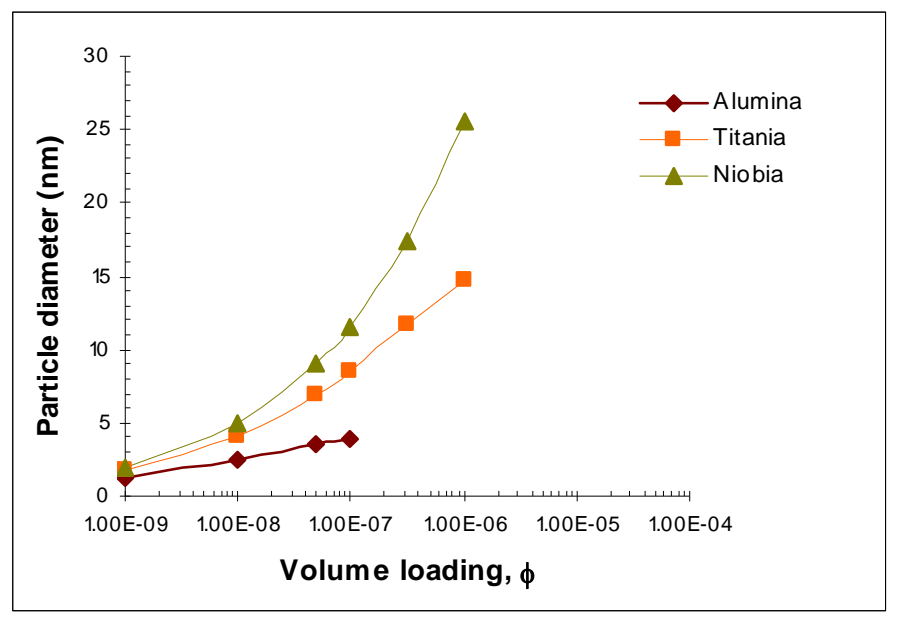

Figure 6.7 Variation of primary particle size of different oxides with particle volume loading $\left(T_{0}=2000 \mathrm{~K}, \kappa=10^{5} \mathrm{Ks}^{-1}\right)$

In the present work various metallic nanoparticles were produced. To apply the model to predict the spark generated particle sizes, two problems should be tackled. Firstly, the exact operating conditions (for example initial temperature, cooling rate, and particle volume loading) in the spark discharge generator are not exactly known. Secondly, there is a lack of information regarding the size dependent properties of the materials (for instance, solid state diffusion coefficients). The results regarding the above-mentioned oxides show that using bulk properties in the computation leads to rather reasonable results at least for first estimates $^{3}$ (see Table 6.2 which shows that the deviations between the calculations and experiments are below 18\%). Thus, in the computation the bulk properties of the metals listed in Table 6.3 are used.

Table 6.3 Properties of the metals (CRC)

\begin{tabular}{|l|l|l|l|l|}
\hline Property & $\mathbf{A u}$ & $\mathbf{C u}$ & $\mathbf{A g}$ & $\mathbf{W}$ \\
\hline Diffusion pre-exponential, $\mathrm{D}_{0}\left(\mathrm{~m}^{2} \mathrm{~s}^{-1}\right)$ & $9.1 \times 10^{-6}$ & $62.1 \times 10^{-6}$ & $27 \times 10^{-6}$ & $42.8 \times 10^{-4}$ \\
\hline Diffusion activation energy, $\mathrm{E}_{\mathrm{a}}\left(\mathrm{kJ} \mathrm{mol}^{-1}\right)$ & 174.5 & 207.3 & 182.8 & 640.5 \\
\hline Surface tension, $\sigma\left(\mathrm{N} \mathrm{m}^{-1}\right)$ & 1.626 & 1.934 & 1.302 & 3.468 \\
\hline Vacancy volume, $\mathrm{v}_{0}\left(\mathrm{~m}^{3}\right)$ & $16.9 \times 10^{-30}$ & $11.8 \times 10^{-30}$ & $17 \times 10^{-30}$ & $15.7 \times 10^{-30}$ \\
\hline Density, $\rho\left(\mathrm{kg} \mathrm{m}^{-3}\right)$ & 19300 & 8920 & 10490 & 19250 \\
\hline Melting temperature $(\mathrm{K})$ & 1337 & 1357 & 1235 & 3695 \\
\hline Boiling temperature $(\mathrm{K})$ & 3129 & 3200 & 2435 & 5828 \\
\hline
\end{tabular}

The operating conditions in the spark generator are estimated with recourse to the literature and applying the values which give comparable results to the electron microscopy observations.

It is known that the plasma, containing the metal vapors, has a relatively high temperature (for example several thousands K), however, it is assumed that the metal vapor condensation and particle formation starts where the temperature has fallen below the boiling temperature of the metal. Therefore, $\mathrm{T}_{0}$ is considered around the boiling point of the metal.

The particle volume loading mainly depends on the electrode material and the characteristics of spark. Our electrical circuit induced erosion of approximately $1-5 \times 10^{13}$ atoms from the electrodes per spark (except for tungsten which was smaller $\sim 10^{12}$ ), consistent with the

\footnotetext{
3 For very small particles of nucleus dimensions (fewer than 100 atoms or molecules), physical and thermodynamic properties differ from those of bulk materials. However, particles are in the nucleus size range only for a negligible period of time (Wu et al., 1993).
} 
literature (Scheeline et al., 1981). These data are used to calculate the particle volume loadings.

The cooling rate is assumed to be in the order of $10^{6}$ to $10^{7} \mathrm{Ks}^{-1}$ (Jenkins and Eagar, 2003; Borghese et al., 1988).

We assume that particles form in a small region around the spark with a volume of approximately $5-20 \times 10^{-10} \mathrm{~m}^{3}$ corresponding to a cylinder of $0.4-0.8 \mathrm{~mm}$ radius and $1 \mathrm{~mm}$ length (the enter-electrode gap distance) ${ }^{4}$. This is in the size range of vapor plume in Figure 6.8 taken by the Schlieren technique (Klueppel and Walters, 1980).

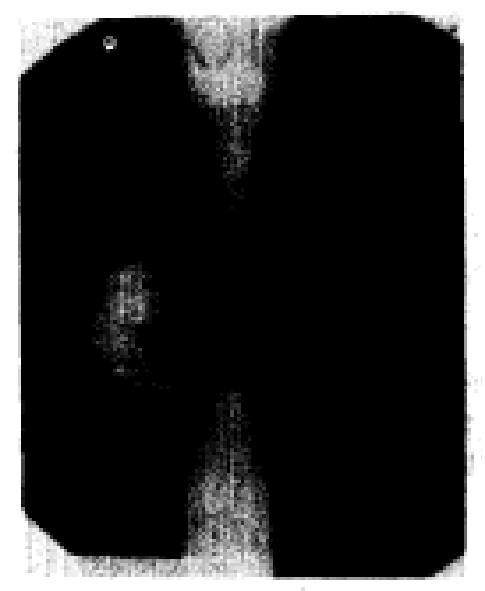

Figure 6.8 Schlieren photograph taken at $210 \mu \mathrm{s}$ with respect to the onset of current in the spark; anode:tungsten, cathode:magnesium, gap:2 $\mathrm{mm}$, gas flow: argon $0.48 \mathrm{Lmin}^{-1}$, frequency: $120 \mathrm{~Hz}$ (by courtesy of Klueppel and Walters, 1980)

Using this technique, Klueppel and Walters (1980) showed that the expansion of the vapor from a spark at atmospheric pressure proceeded from an initial narrow inter-electrode filament, resulting in the formation of a toroidally-shaped structure coaxially enveloping the spark at long enough times after the current has decayed to negligible values ${ }^{5}$. It should be emphasized that applying a constant particle volume loading in the calculation is a rough, simplifying assumption. The particle volume loadings are calculated using this assumption and the electrode mass loss data of chapter two.

Table 6.4 shows the particle sizes calculated on the basis of the above-mentioned assumptions. The particle sizes vary in a rather small range $(3.7-5.3 \mathrm{~nm})$, consistent with the size measurements presented in the previous chapters.

Table 6.4 Model prediction for the metals ( $T_{0} \sim$ boiling point of the metal $K, \kappa=5 \times 10^{6} \mathrm{Ks}^{-1}$ )

\begin{tabular}{|l|l|l|l|}
\hline Metal & $\Phi$ & Size nm (model) & Size nm (TEM) \\
\hline $\mathrm{Au}$ & $3.2 \times 10^{-7}$ & 4.6 & 5 \\
\hline $\mathrm{Cu}$ & $3.11 \times 10^{-7}$ & 5.3 & 5 \\
\hline $\mathrm{Ag}$ & $2.8 \times 10^{-7}$ & 4 & 4 \\
\hline $\mathrm{W}$ & $6.34 \times 10^{-8}$ & 3.7 & 4 \\
\hline
\end{tabular}

\footnotetext{
4 Light scattering measurements indicate that substantial numbers of atoms condense into particles in the vicinity of the spark (Scheeline et al., 1981).

5 Presence of free atoms in the post discharge environment for at least $200 \mu$ s had also been previously detected by absorption measurements (Scheeline et al., 1981).
} 
Figure 6.9 shows a typical primary particle size evolution with temperature for gold at the presumed operating conditions. Figure 6.9 reveals that the particles reach their final sizes in about $400 \mu \mathrm{s}$.

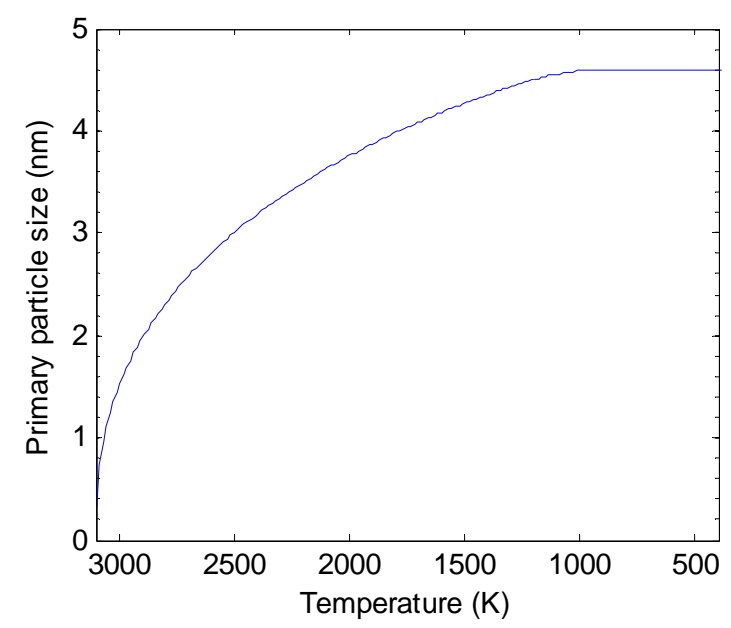

Figure 6.9 Primary particle size predicted for $\mathrm{Au}\left(T_{0}=3100 \mathrm{~K}, \kappa=5 \times 10^{6} \mathrm{Ks}^{-1}, \Phi=3.2 \times 10^{-7}\right)$

\subsection{Conclusion}

In order to predict the primary particle sizes, the model derived by Lehtinen et al. (1996) was solved numerically. Using the material properties given by Windeler et al. (1997), and typical operating conditions in a flame reactor given by Bandyopadhyaya et al. (2003), the model was tested by calculating the characteristic times and the particle sizes for three oxides (niobia, titania, and alumina). According to the model, the particles reach their final size at a temperature about $70-80 \%$ of the bulk melting points. High volume loading and low cooling rate result in larger particles. The model predicts a small variation of particle sizes from material to material for the high quenching rates and thus gives a reasonable explanation for the relatively small size variations observed experimentally in the spark discharge generator.

The particle sizes of the metals applied in the present study were approximated using their bulk properties and the operation conditions in the spark generator were estimated based on other sources. These estimates were crude, but particle sizes comparable with the measurements were obtained. With the presumed conditions, particles reach their final sizes in several hundreds of micro seconds. This implies that each spark may be regarded as an individual event below $f \sim 1000 \mathrm{~Hz}$.

\section{References}

Bandyopadhyaya R., A. Lall, S. K. Friedlander, Aerosol Dynamics and the Synthesis of Fine Solid Particles, Powder Technology, Volume 139, 2004, pp. 193-199

Baronets P. N., Cylindrical Shock Waves Generated by a Spark Discharge and Their Interaction With The Shock Waves From a Pulsed Induction Discharge, Fluid Dynamics, Volume 29, Number 1, 1994, pp. $129-132$ 
Borghese A., A. D’Alessio, M. Diana, C. Venitozzi, Development of Hot Nitrogen Kernel Produced by a Very Fast Spark Discharge, Twenty-Second Symposium (International) on Combustion/ The Combustion Institute, 1988, pp. 1651-1659

Ding Feng, Arne Rosen, Kim Bolton, Size Dependence of the Coalescence and Melting of Iron Clusters: A Molecular-Dynamics Study, Physical Review B, Volume 70, 2004, pp. 075416-6

Flagan R. C., S. K. Friedlander, Particle Formation in Pulverized Coal Combustion- a Review in Recent Developments in Aerosol Science, Wiley, New York, 1978

Granqvist C. G., R. A. Buhrman, Ultrafine Metal Particles, Journal of Applied Physics, Volume 47, Number 5, 1976, pp. 2200-2219

Hawa T., M. R. Zachariah, Coalescence Kinetics of Unequal Sized Nanoparticles, Journal of Aerosol Science, Volume 37, 2006, pp. 1-15

Jenkins Niel T., Thomas W. Eagar, Submicron Particle Chemistry: Vapor Condensation Analogous to Liquid Solidification, JOM Journal of the Minerals, Metals and Materials Society, Volume 55, Number 6, 2003, pp. 44-47

Klueppel R. J., J. P. Walters, Observations on the Formation of Spark Discharge and its Influence by an External Magnetic Field, Spectrochimica Acta, Volume 35 B, 1980, pp. 431-446

Klueppel R. J., D. M. Coleman, J. W. Hosch, J. P. Walters, Structures Detected in a Stable, High-Voltage Spark Discharge by Time-Resolved Schlieren Spectroscopy, Spectrochimica Acta, Volume 33 B, 1978, pp. 741-752

Koch Carl C., Nanostructured Materials, William Andrew, 2007

Koch W., S. K. Friedlander, The Effect of Particle Coalescence on the Surface Area of a Coagulating Aerosol, Journal of Colloid and Interface Science, Volume 140, Number 2, 1990, pp. 419-427

Lee K. W., H. Chen, J. A. Gieseke, Log-Normally Preserving Size Distribution for Brownian Coagulation in the Free- Molecule Regime, Aeroaol Science and technology, Volume 3, 1984, pp. 53-62

Lehtinen Kari E. J., Robert S. Windeler, Sheldon K. Friedlander, Prediction of Nanoparticle Size and Onset of Dendrite Formation Using the Method of Characteristic Times, Journal of Aerosol Science, Volume 27, Number 6, 1996, pp. 883-896

Lehtinen Kari E. J., Robert S. Windeler, Sheldon K. Friedlander, a Note on the Growth of Primary Particles in Agglomerate Structures by Coalescence, Journal of Colloid and Interface Science, Volume 182, 1996, pp. 606608

Lehtinen Kari E.J., Michael R. Zachariah, Effect of Coalescence Energy Release on the Temporal Shape Evolution of Nanoparticles, Physical Review B., Volume 63, 2001, pp. 255402-7

Lehtinen Kari E.J., Michael R. Zachariah, Energy Accumulation in Nanoparticle Collision and Coalescence Processes, Journal of Aerosol Science, Volume 33, 2002, pp. 357-368

Lide David R., CRC Handbook of Chemistry and Physics, 89 th Edition, Taylor \& Fransis, Inc., 2008

Miller Richard K., Introduction to Differential Equations, Prentice-Hall International Editions, 1987

Nakaso Koichi, Manabu Shimada, Kikuo Okuyama, Knut Deppert, Evaluation of the Change in the Morphology of Gold Nanoparticles During Sintering, Journal of Aerosol Science, Volume 33, 2002, pp. 1061-1074

Panda S., S. E. Pratsinis, Modeling the Synthesis of Aluminum Particles by Evaporation-Condensation in an Aerosol Flow Reactor, Nanostructured Materials, Volume 5, Number 7/8, 1995, pp. 755-767 
Schmidt-Ott A., New Approach to In Situ Characterization of Ultrafine Agglomerates, Journal of Aerosol Science, Volume 19, Number 5, 1988, pp. 553-563

Seipenbusch M., A. P. Weber, A. Schiel, G. Kasper, Influence of the Gas Atmosphere on Restructuring and Sintering Kinetics of Nickel and Platinum Aerosol Nanoparticle Agglomerates, Journal of Aerosol Science, Volume 34, 2003, pp. 1699-1709

Scheeline A., J. A. Norris, J. C. Travis, R. DeVoe, J P. Walters, Particulates Formed by a Stabilized High Voltage Spark Discharge, Spectrochimica Acta, Volume 36 B, Number 4, 1981, pp. 373-383

Shimada Manabu, Takafumi Seto, Kikuo Okuyama, Size Change of Very Fine Silver Agglomerates by Sintering in Heated Flow, Journal of Chemical Engineering of Japan, Volume 27, Number 6, 1994, pp. 795-802

Simchi A., R. Ahmadi, S. M. Seyed Reihani, A. Mahdavi, Kinetics and Mechanisms of Nanoparticle Formation and Growth in Vapor Phase Condensation Process, Materials and Design, Volume 28, 2007, pp. 850-856

Singh Yogendra, Julie R. N. Javier, Shery H. Ehrman, Martin H. Magnusson, Knut Deppert, Approaches to Increasing Yield in Evaporation-Condensation Nanoparticle Generation, Journal of Aerosol Science, Volume 33, 2002, pp. 1309-1325

Weber A. P., S. K. Friedlander, in Situ Determination of the Activation Energy for Restructuring of Nanometer Aerosol Agglomerates, Journal of Aerosol Science, Volume 28, 1997, pp. 179-192

Windeler, R. S., K. E. J. Lehtinen, S. K. Friedlander, Production of Nanometer-Sized Metal Oxide Particles by Gas Phase Reaction in a Free Jet. II: Particle Size and Neck Formation-Comparison with Theory, Aerosol Science and Technology, Volume 27, 1997, pp. 191-205

Wu M. K., R. S. Windeler, C. K. R. Steiner, T. Bors, Controlled Synthesis of Nanosized Particles by Aerosol Processes, Aerosol Science and Technology, Volume 19, 1993, pp. 527-548

Wu M. K., S. K. Friedlander, Enhanced Power Law Agglomerate Growth in the Free Molecule Regime, Journal of Aerosol Science, Volume 24, 1993, pp. 273-282

Zachariah M. R., M. J. Carrier, Properties of Silicon Nanoparticles: A Molecular Dynamics Study, Journal of Physical Chemistry, Volume 100, 1996, pp. 14856

Zachariah M. R., M. J. Carrier, Molecular Dynamics Computation of Gas-Phase Nanoparticle Sintering: a Comparison with Phenomenological Models, Journal of Aerosol Science, Volume 30, Number 9, 1999, pp. $1139-1151$ 


\section{Summary}

Spark discharge is a method for producing nanoparticles from conductive materials. Besides the general advantages of nanoparticle synthesis in the gas phase, the method offers additional advantages like simplicity, compactness and versatility. The synthesis process is continuous and is performed at atmospheric pressure, which is quite economic in comparison with vacuum methods and has the potential of being scaled up. In addition, it bears unparalleled possibilities of mixing materials. Although the method has extensively been used for research purposes, there is a lack of systematic study in previous work. This is the main objective of the present thesis.

The investigation on the performance of the spark discharge generator revealed that the mean particle size can be tailored via the energy per spark and the particle production rate can be controlled by the spark energy and the spark frequency. Different electrode materials led to different mass production rates, where thermal conductivity, evaporation enthalpy and the boiling point had a major influence.

Characterization of the spark generated particles revealed information on sintering, oxidation and structure on all length scales. Besides the nanoparticles, much larger particles are sometimes formed. While the former are formed by vaporization and condensation, the latter arise from dispersion of molten pools on the electrode surface. Agglomeration and sintering of nanoparticles in gas suspension and on substrates are observable through transmission electron microscopy (TEM), surface determination by adsorption (BET) and mobility analysis, as well as through comparison of the characteristic diameters these methods give. Inter-particle sintering at room temperature was observed and indicated complete absence of adsorbed surface layers. This is an effect of the extreme purity achievable with the spark discharge method. For non-noble metals, oxygen impurities in the carrier gas led to oxide layers on the particle surfaces, which hindered sintering.

The merit of the spark generation nanoparticle synthesis process in achieving specific functionalities was demonstrated by examples of successful application. Spark generated Pd nanoparticles showed a hydrogen storage capacity near the theoretical maximum value. Spark generated $\mathrm{Mg}$ nanoparticles exhibited a decrease in the hydrogen desorption temperature, which makes them an interesting starting point for new hydrogen storage systems. Furthermore, nanoparticles from spark discharge proved to be interesting materials for production of lithium ion battery electrodes.

Flexible mixing of materials on a nano scale down to the atomic scale should give access to an enormous variety of new material properties tunable not only through size but also through mixing ratios. Thus, there is a great interest in developing new methods for synthesis of mixed nanoparticles. The ability of spark discharge to generate mixed metallic and composite nanoparticles is expected to open new fields of application. Electrodes with different degrees of premixing of the constituents were applied to study the composition, texture and structure of the nanoparticulate product. Various miscible and immiscible systems with small and large differences in evaporation rates were studied. In almost all the cases, x-ray diffraction or compositional analysis of the single particles showed evidence of mixing. Using different metals for the positive and the negative electrode it was shown that spark discharge induces mixing, and on the example of the Au-Pd system the effect of operating conditions on mixing was studied. It was observed that the mixing ratio is mainly influenced by the polarity and by the gap distance between the electrodes.

The particles produced from various materials are mainly very small compared to other production methods, a few $\mathrm{nm}$ in diameter, and in a rather narrow size range. Surprizingly, the size does not show any significant material dependence. A mathematical model derived 
by Lehtinen et al. (1996) was applied to explain the experimental findings and solved numerically. The model shows agreement with the sizes observed and qualitatively explains the small sizes as a consequence of the large cooling rate in the spark discharge process. 


\section{Samenvatting}

Spark discharge generation is een methode om nanodeeltjes uit geleidende materialen te produceren. Naast de algemene voordelen van nanodeeltjes synthese in de gasfase biedt deze methode extra voordelen zoals eenvoud, compactheid en veelzijdigheid. Het syntheseproces is continu en wordt bij atmosferische druk uitgevoerd, waardoor het vrij goedkoop is in vergelijking met vacuümmethodes. Ook is opschaling van het proces mogelijk. Bovendien biedt deze methode ontelbare mogelijkheden om materialen te mengen. Hoewel de methode uitgebreid voor onderzoekdoeleinden is gebruikt, is er tot nu toe een gebrek aan systematische studie. Dit is de belangrijkste doelstelling van de huidige thesis.

Onderzoek naar de prestaties van de spark generator heeft aangetoond dat de gemiddelde deeltjesgrootte onder andere afhankelijk is van de energie die vrij komt tijdens het vonkproces en dat de deeltjesproductiesnelheid kan worden gecontroleerd door de vonkenenergie en de vonkfrequentie. Verschillende elektrodematerialen leidden tot verschillende massaproductiesnelheden waarbij het warmtegeleidingsvermogen, de verdampingsenthalpie en het kookpunt de grootste invloed hadden.

Karakterisering van de met spark discharge gegenereerde deeltjes leverde informatie op over sintering, oxidatie en structuur op alle lengteschalen. Naast nanodeeltjes worden soms veel grotere deeltjes gevormd. Terwijl de eerstgenoemden worden gevormd door verdamping en condensatie, ontstaan de laatstgenoemden door dispersie van gesmolten pools op het elektrodeoppervlak. De agglomeratie- en sinteringsprocessen van nanodeeltjes in gassuspensies en op substraten zijn waarneembaar door transmissie-elektronenmicroscopie (TEM), oppervlaktebepaling door adsorptie (BET) en mobiliteitsanalyse, evenals door vergelijking van de karakteristieke deeltjesdiameters die deze methoden geven. Er werd interparticle sintering waargenomen bij kamertemperatuur, wat wees op het volledig ontbreken van geadsorbeerde oppervlaktelagen. Dit is een effect van de extreme zuiverheid die kan worden bereikt met de spark discharge methode. Voor niet-edele metalen leidden de zuurstofonzuiverheden in het draaggas tot oxidelagen op de deeltjesoppervlakken, wat het sinteringsproces belemmerde.

De geschiktheid van het productieproces van nanodeeltjes via spark discharge om specifieke functionaliteiten te bereiken werd aangetoond met voorbeelden van succesvolle toepassingen. Via spark discharge gegenereerde palladium nanodeeltjes lieten een waterstofopslagcapaciteit zien die dichtbij de theoretische maximumwaarde ligt. Via spark discharge genereerde magnesium nanodeeltjes veroorzaakten een daling van de waterstofdesorptietemperatuur, waarmee deze nanodeeltjes een interessant uitgangspunt zijn voor nieuwe systemen van waterstofopslag. Verder hebben deze nanodeeltjes bewezen interessant te zijn als materiaal voor de productie van elektroden van lithium-ionbatterijen.

Het flexibel mengen van materialen op nanoschaal tot aan de atomaire schaal zou toegang moeten geven tot een enorme verscheidenheid aan nieuwe materiaaleigenschappen die niet alleen via de deeltjesgrootte maar ook via de mengverhoudingen te beïnvloeden zijn. Daarom is er grote belangstelling voor het ontwikkelen van nieuwe methodes voor de synthese van gemengde nanodeeltjes. De mogelijkheid van spark discharge om gemengde metallische en samengestelde nanodeeltjes te produceren opent naar verwachting nieuwe toepassingsgebieden. Elektroden met verschillende mengverhoudingen van constituenten werden toegepast om de samenstelling, textuur en structuur van het nanoparticulaire product te bestuderen. Diverse mengbare en niet-mengbare systemen met kleine en grote verschillen 
in verdampingssnelheden werden bestudeerd. In bijna alle gevallen leverde röntgendiffractie of samenstellingsanalyse van de afzonderlijke deeltjes bewijsmateriaal op voor menging. Door verschillende metalen te gebruiken voor de positieve en negatieve elektrodes werd aangetoond dat spark discharge menging induceert, en in het geval van het Au-Pd-systeem werd het effect van operationele condities op de menging bestudeerd. Er werd waargenomen dat de mengverhouding hoofdzakelijk wordt beïnvloed door de polariteit en door de afstand tussen de elektroden.

In vergelijking met andere productiemethodes zijn de deeltjes die uit meerdere materialen worden geproduceerd meestal zeer klein: een paar nanometer in diameter en met een geringe spreiding in deeltjesgrootte. Verrassend genoeg vertoont de deeltjesgrootte geen significante materiaalafhankelijkheid. Een door Lehtinen et al. (1996) afgeleid wiskundig model werd toegepast om de resultaten te verklaren en werd numeriek opgelost. Het model toont overeenkomst met de waargenomen deeltjesgrootten en geeft een kwalitatieve verklaring voor de geringe grootten als zijnde het gevolg van de grote koelsnelheid in het spark generation-proces. 
Appendix A

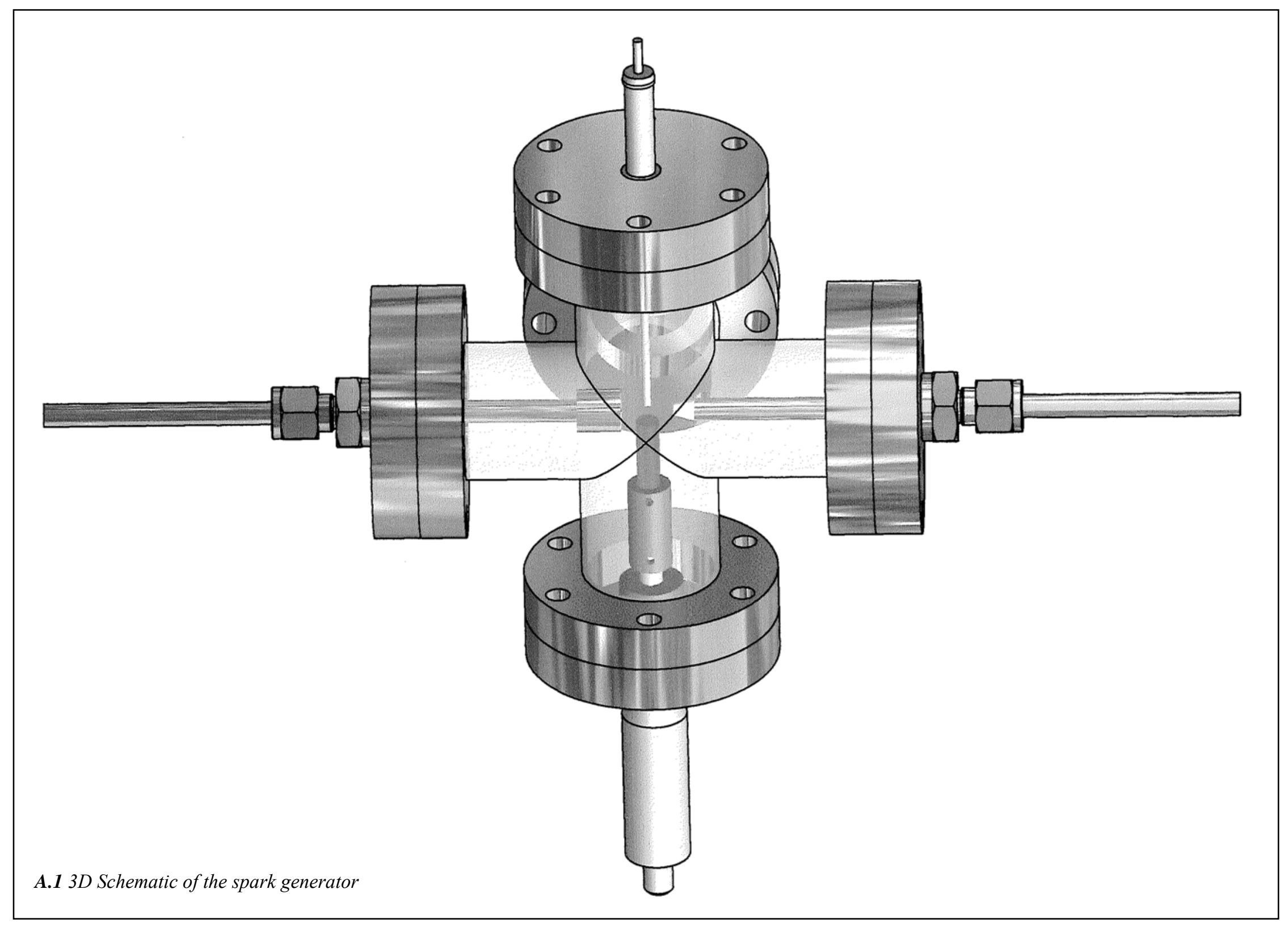


Appendix A

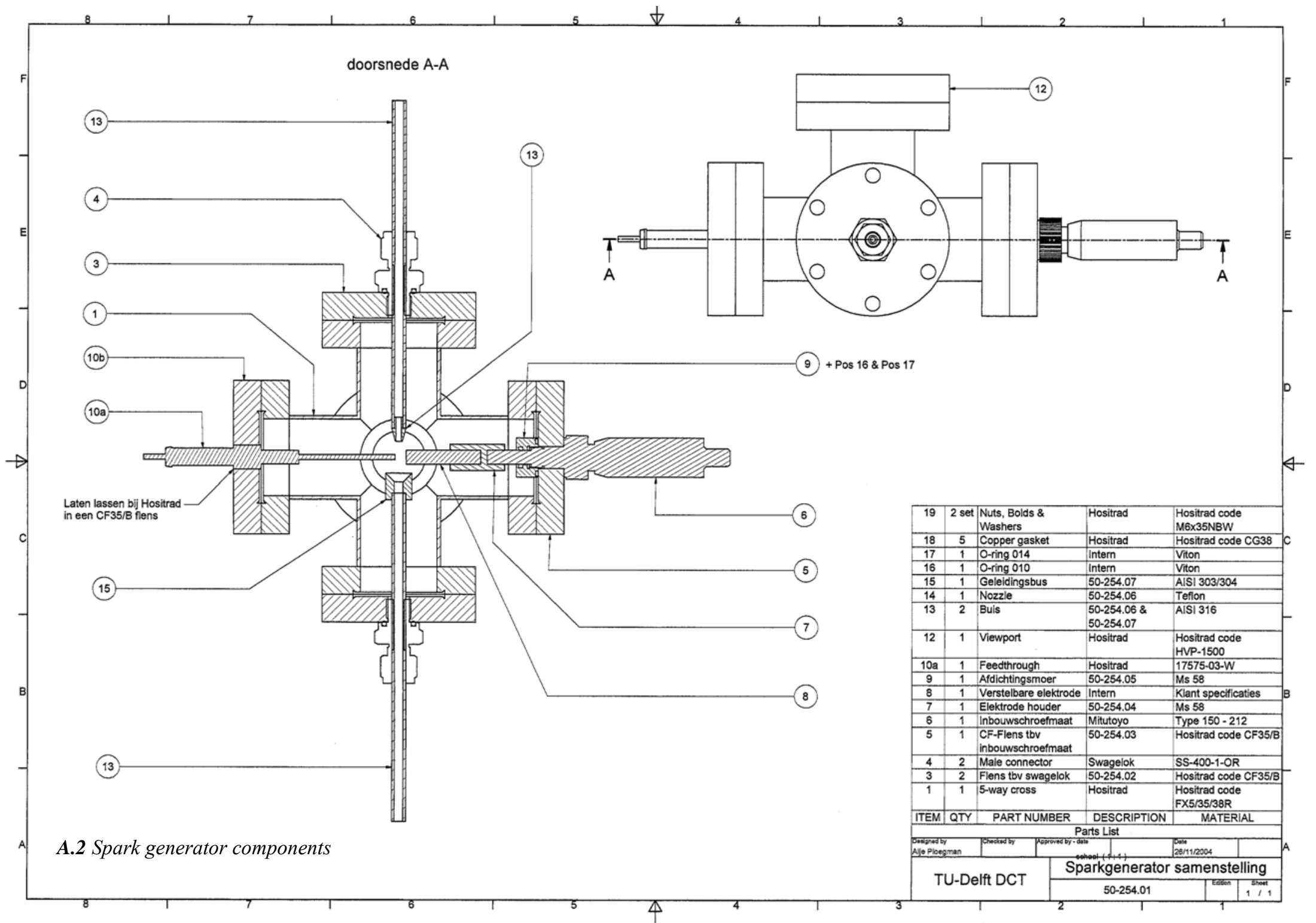




\section{B.1 Differential Mobility Analyzer (DMA)}

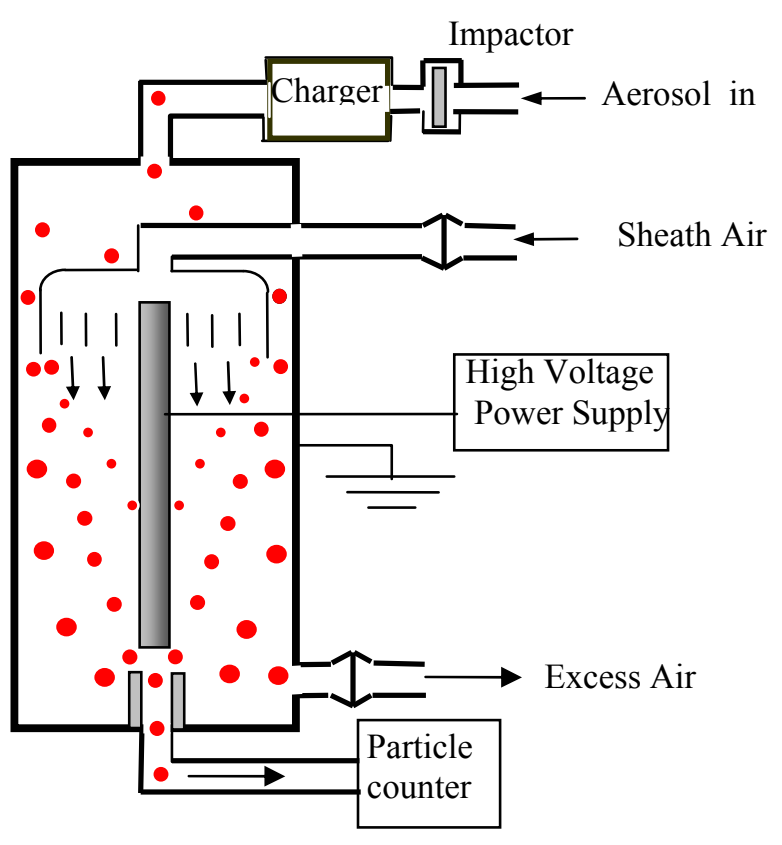

Figure B.1 Typical differential mobility analyzer (reproduced from TSI DMA manual)

The differential mobility analyzer is mainly utilized for size selecting particles from polydisperse aerosol and producing a monodisperse aerosol. In combination with a particle sensor (condensation particle counter, aerosol electrometer..) it can be used to measure the size distribution of a polydisperse aerosol. In the DMA, particles are classified due to their electrical mobility. As shown in Figure B.1, the DMA consists of two concentric metal cylinders. The particle free sheath air enters the DMA at the top and passes through the flow straightener down to the annular space between the cylinders. The polydisperse aerosol flow is also introduced to the DMA at the top surrounding the inner core of the sheath air flow without mixing of two laminar streams. The inner rod is maintained at a controlled voltage while the outer cylinder is electrically grounded creating an electric field which attracts charged particles from the aerosol layer at the outer wall and causes them to drift across the clean air flow toward the center rod. Particles with a high electric mobility deposit on the upper part of the central rod. Particles with low electrical mobility are carried out by excess air. Only particles within a narrow mobility range exit the small slit at the bottom of the collector rod. This mobility can be selected by varying the voltage applied to the central rod according to the equation

$$
Z=\frac{q_{\text {sheath }} \ln \left(r_{2} / r_{1}\right)}{2 \pi V L}
$$

$\mathrm{Z}$ is the electrical mobility of the particles $\left(\mathrm{m}^{2} / \mathrm{V}-\mathrm{s}\right), \mathrm{q}_{\text {sheath }}$ is the sheath air flow rate $\left(\mathrm{m}^{3} / \mathrm{s}\right)$, $\mathrm{r}_{1}, \mathrm{r}_{2}$ are the radii of the annular gap space, $\mathrm{V}$ (volt) is the voltage applied on the collector rod, and $\mathrm{L}(\mathrm{m})$ is the length of the center rod between the aerosol entrance and the slit. For a known charge distribution on particles a unique electrical mobility is associated with every particle size (Hinds, 1999): 


$$
Z=\frac{n e C_{c}}{3 \pi \mu d_{p}}
$$

Where $d_{p}(m)$ is the particle size, $n$ number of elementary charges on the particles, e is the electronic charge $\left(1.6 \times 10^{-19} \mathrm{C}\right), \mu$ is the gas viscosity $(\mathrm{Pa}-\mathrm{s}), \lambda(\mathrm{m})$ is the mean free path of the gas and $\mathrm{C}_{\mathrm{c}}$ is the Cunningham slip correction factor. Small particles whose size approaches the mean free path of the gas settle faster than predicted by Stokes' law, because the relative velocity of the gas at the surface of the particle is not zero and there is a slip at the particle surface. To account for this effect the Cunningham correction factor is given as (Hinds, 1999):

$$
C_{c}=1+\frac{\lambda}{d_{p}}\left[2.514+0.8 \exp \left(-0.55 \frac{d_{p}}{\lambda}\right)\right]
$$

Table B.1 Dimensions of DMAs used in this study

\begin{tabular}{|l|l|l|l|}
\hline Type & $\mathrm{L}(\mathrm{cm})$ & $\mathrm{r}_{1}(\mathrm{~cm})$ & $\mathrm{r}_{2}(\mathrm{~cm})$ \\
\hline Short DMA & 11.4 & 0.935 & 1.96 \\
\hline Long DMA & 36.4 & 0.935 & 1.96 \\
\hline TSI DMA & 44.44 & 0.937 & 1.958 \\
\hline
\end{tabular}

The length is considered from middle of inlet slit to the middle of outlet slit

Table B.1 lists the dimensions of the DMAs used in the present study.

Figure B.2 shows the relationship between the particle size and the voltage applied to the central rod of the short DMA.

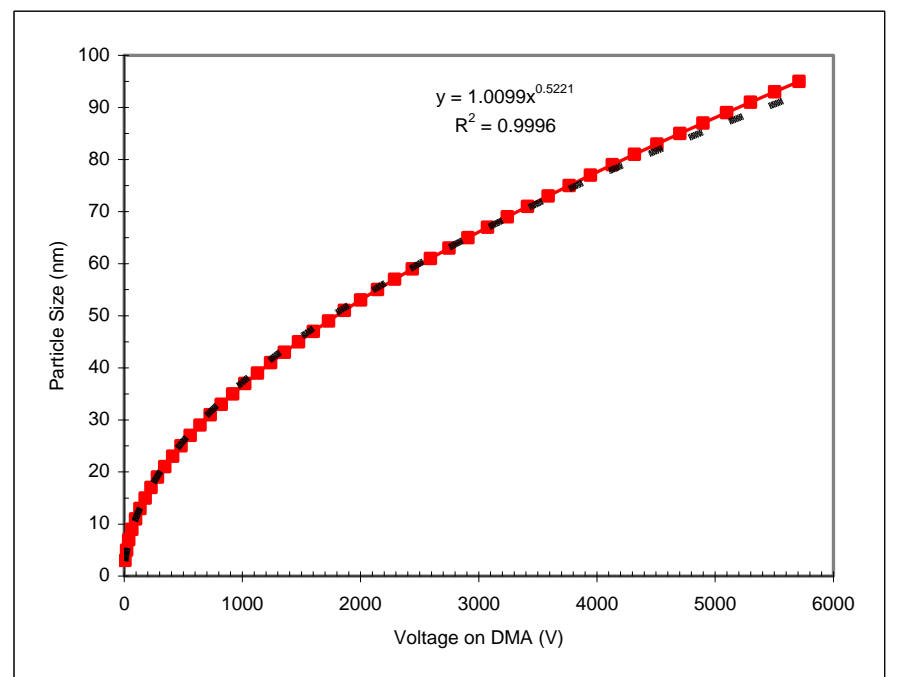

Figure B.2 Particle sizes selected at different applied voltage on the short DMA (10 lpm sheath and excess air flows, $1.5 \mathrm{lpm}$ aerosol flow)

Under certain assumptions, the particle motion in the DMA is governed by single, first order, exact differential equation which can be solved analytically (Knutson, 1975, I). 


\section{B.2 Transfer Function of DMA}

The transfer function $(\Omega)$ describes the probability that a particle of mobility Z, which enters the mobility analyzer through the polydisperse aerosol flow will leave the analyzer via the slit in the monodisperse flow. It can be obtained either theoretically or empirically. To obtain the empirical transfer function of a DMA, two identical DMAs can be connected in series and the particle concentration at the outlet of the second DMA be scanned (Fissan, 1996).

$\Omega=\left(1 / q_{\text {poly }}\right) \cdot \max \left[0, \min \left(q_{\text {polyl }}, q_{\text {mono }},\left[1 / 2\left(q_{\text {poly }}+q_{\text {mono }}\right)-\left|2 \pi Z_{p} \cdot \Delta \Phi+1 / 2\left(q_{\text {excess }}+q_{\text {sheath }}\right)\right|\right]\right)\right]$

A basic integral transform which relates the DMA output, $N^{*}(V)$, to the parameters of the input aerosol, $N$ and $f\left(Z_{p}\right)$, is given by (Knutson, 1975, II):

$N^{*}(V)=N q_{\text {poly }} \int_{0}^{\chi} \Omega\left(\Lambda Z_{p} V\right) f\left(Z_{p}\right) d Z_{p}$

Where:

$V=$ Center rod voltage of the mobility analyzer

$\Delta \Phi=\Lambda V$

$\Lambda=\mathrm{L} / \ln \left(\mathrm{r}_{2} / \mathrm{r}_{1}\right)$

$N=$ Number concentration of the aerosol

$N^{*}(V)=$ Number of particles exiting the DMA through monodisperse flow per unit time

$Z_{\mathrm{p}}=$ Particle electric mobility with differential distribution function of $f\left(Z_{p}\right)$

Only particles emerge via the monodisperse flow that obey

$\left(q_{\text {excess }}+q_{\text {sheath }}\right)-\left(q_{\text {polyl }}+q_{\text {mono }}\right)<4 \pi \Lambda V Z_{p}<\left(q_{\text {excess }}+q_{\text {sheath }}\right)+\left(q_{\text {poly }}+q_{\text {mono }}\right)$

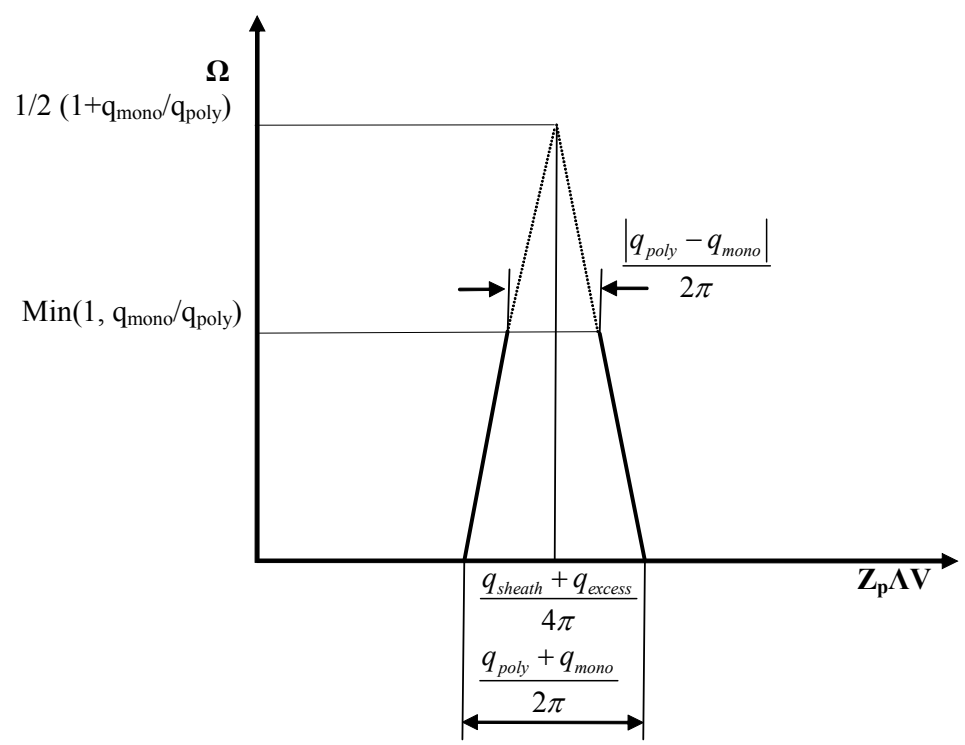

Figure B.3 The transfer function of mobility analyzer (Knutson and Whitby, 1975) 
For the condition of equal polydisperse aerosol flow and mono disperse aerosol flow, the height of the ideal triangle transfer function will be unit.

The transfer function is characterized by the center mobility of the band pass as

$$
z_{p}^{*}=\frac{q_{\text {sheath }}+q_{\text {excess }}}{4 \pi \Lambda V}
$$

And particles selected by the DMA are characterized by the mobility half width $\left(\Delta Z_{p}\right)$, given by (TSI DMA manual)

$$
\Delta Z_{p}=\frac{q_{\text {poly }}+q_{\text {mono }}}{4 \pi \Lambda V}
$$

The resolution is given by

$$
\frac{\Delta Z_{p}}{Z_{p}^{*}}=\frac{q_{\text {poly }}+q_{\text {mono }}}{q_{\text {sheath }}+q_{\text {excess }}}
$$

Equation (B.9) shows that the resolution is strongly affected by the flow settings.

In the DMA the width of the transfer function can be broadened by diffusion of the small particles, while conserving its area. The particle losses due to deposition on the walls may lower the concentration of these particles while conserving the distribution width. 


\section{B.3 Bipolar Diffusion Charging of Aerosol Particles}

Differential mobility analysis is the technique mostly used to determine the particle size distribution of submicometer particles which is based on the electrical properties of charged particles. For this purpose the aerosol particles are first charged in a pipolar charger and then the electrical mobility distribution of the particles is measured by a DMA. There is a unique electrical mobility associated with every particle size carrying a known number of charge (Hinds, 1999). To achieve an accurate particle size distribution, the bipolar charge equilibrium of the aerosol particles has to be well known.

The charge distribution of an aerosol in charge equilibrium with bipolar ions can be represented by the Boltzmann equilibrium charge distribution (Hinds, 1999)

$$
f_{n}=\frac{\exp \left(-K_{E} n^{2} e^{2} / d_{p} k T\right)}{\sum_{n=-\infty}^{\infty} \exp \left(-K_{E} n^{2} e^{2} / d_{p} k T\right)}
$$

$f_{n} \quad$ fraction of particles of a given size

$\mathrm{n}$ number of elementary unit of charge

e charge of electron $1.6 \times 10^{-19}(\mathrm{C})$

$\mathrm{K}_{\mathrm{E}} \quad 9.0 \times 10^{9}\left(\mathrm{~N} \cdot \mathrm{m}^{2} / \mathrm{C}^{2}\right)$

$\mathrm{T}$ absolute temperature $(\mathrm{K})$

k Boltzmann constant $1.38 \times 10^{-23}(\mathrm{~J} / \mathrm{K})$

$\mathrm{d}_{\mathrm{p}}$ particle diameter $(\mathrm{m})$

An aerosol reaches the Boltzmann equilibrium charge distribution with the rate of (Hinds, 1999)

$$
\frac{n(t)}{n_{0}}=\exp \left(-4 \pi K_{E} e Z_{i} N_{i} t\right)
$$

Here $\mathrm{n}(\mathrm{t})$ is the number of charges on a particle after exposure to the bipolar ion concentration $\mathrm{N}_{\mathrm{i}}$ for a time $\mathrm{t}$, and $\mathrm{n}_{0}$ is the initial charge at $\mathrm{t}=0$. The time for an aerosol to reach the Bolzmann equilibrium depends on the product $\mathrm{N}_{\mathrm{i}}$ t (Hinds, 1999). For particles smaller than $50 \mathrm{~nm}$ the Boltzmann equation underestimates the fraction of charged particles.

The differential equation for the diffusion of gaseous ions of a certain sign towards a spherical particle in the absence of an external electric field is (Fuchs, 1963):

$$
\frac{\partial n}{\partial t}=D \frac{\partial^{2} n}{\partial \rho^{2}}+\frac{2 D}{\rho} \frac{\partial n}{\partial \rho}+B \frac{\partial}{\partial \rho}\left[\rho^{2} n F(\rho)\right]
$$

where:
$\rho$ the distance from the center of the particle
$\mathrm{n}$ concentration of the ions
D diffusion coefficient of ions
B ion mobility
F electrostatic force acting on the ion 
If $\frac{\partial n}{\partial t}=0$, the steady flux of ion towards the particle is (Fuchs, 1963):

$I=4 \pi \rho^{2}\left(D \frac{\partial n}{\partial \rho}-B F n\right)=$ const.

at $\rho=\infty, n=n_{0}$

$n(\rho)=\exp \left[-\frac{B}{D} \varphi(\rho)\right]\left\{n_{0}+\frac{I}{4 \pi D} \int_{\infty}^{\rho} \frac{1}{\rho^{2}} \exp \left[\frac{B}{D} \varphi(\rho)\right] d \rho\right\}$

where:

$\varphi(\rho)=\int_{\rho}^{\infty} F(\rho) d \rho=\frac{i \varepsilon^{2}}{\rho}-\frac{\varepsilon^{2} a^{3}}{2 \rho^{2}\left(\rho^{2}-a^{2}\right)}$

$\varphi(\rho)$ potential energy of the ion

$\varepsilon \quad$ elementary charge

$i \varepsilon \quad$ particle charge (if the charges of the ions and the particle are of the same sign, $\mathrm{i}$ is positive)

a particle radius

It is assumed that the particle is surrounded by a concentric limiting sphere with radius (Fuchs, 1963)

$\delta=a+\lambda^{\prime}$

and the ions moving inside it travel without collisions with gas molecules. $\lambda$ is the free mean path of gaseous ions and $\lambda^{\prime}$ is the mean distance from the surface of the particle at which the ions collide with gas molecules for the last time before striking this surface (Fuchs, 1963).

$\frac{\delta}{a}=\frac{a^{2}}{\lambda^{2}}\left[\frac{\left(1+\frac{\lambda}{a}\right)^{5}}{5}-\frac{\left(1+\frac{\lambda^{2}}{a^{2}}\right)\left(1+\frac{\lambda}{a}\right)^{3}}{3}+\frac{2}{15}\left(1+\frac{\lambda^{2}}{a^{2}}\right)^{5 / 2}\right]$

The number of ions striking the particle per unit of time, in the absence of electrostatic forces is:

$I^{\prime}=4 \pi \delta^{2} n(\delta) \frac{\bar{c}}{4}\left(\frac{a}{\delta}\right)^{2}$

Here $n(\delta)$ is the ion concentration on the limiting sphere, and $\bar{c}$ is mean thermal velocity of the ions, $4 \pi \delta^{2} n(\delta) \frac{\bar{c}}{4}$ is the number of ions emerging from the limiting sphere and $\left(\frac{a}{\delta}\right)^{2}$ is the fraction of ions reaching the particle. The flux of ions moving in a given direction is proportional to the cosine of the angle between this direction and the normal to the limiting sphere (Figure B.4). In the presence of electrostatic forces this fraction $\alpha>(a / \delta)^{2}$ in the case of attraction and $\alpha<(a / \delta)^{2}$ in the case of repulsion. 


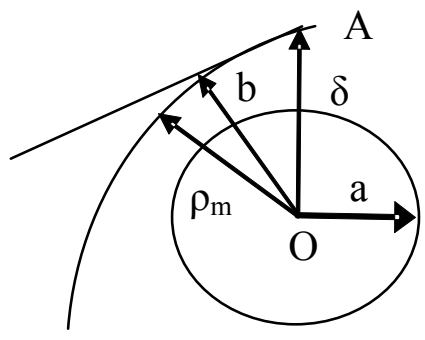

Figure B.4 Moving of ions inside the limiting sphere (Fuchs, 1963)

To determine $\alpha$, the minimal distance $\rho_{\mathrm{m}}$ must be found between the particle center $\mathrm{O}$ and the trajectory of the ion emerging from the point A on the limiting sphere and moving with the mean thermal kinetic energy (Fuchs, 1963).

$b^{2}=\rho_{m}^{2}\left\{1+\frac{2}{3 k T}\left[\varphi(\delta)-\varphi\left(\rho_{m}\right)\right]\right\}$

$\mathrm{b}$ is distance from $\mathrm{O}$ to the tangent drawn to the trajectory at point $\mathrm{A}$. If $\mathrm{b}$ has a minimum $\mathrm{b}_{\mathrm{m}}$, only those ions can reach the particle for which $\mathrm{b} \leq \mathrm{b}_{\mathrm{m}}$ then $\alpha=\left(b_{m} / \delta\right)^{2}$ for positive $\mathrm{i}$ and for negative $i, \alpha=1$ for all values of a.

Table B.2 Comparison of calculated ( $\alpha$ ) coefficients in this work (top) with Fuch 's calculations

\begin{tabular}{|c|c|c|c|c|c|c|c|}
\hline & \multicolumn{7}{|c|}{$\left(\lambda=1.3 \times 10^{-6}\right)$} \\
\hline $\begin{array}{l}a(\mathrm{~cm}) \\
\mathbf{i}\end{array}$ & $0.5 \times 10^{-7}$ & $10^{-7}$ & $3 \times 10^{-7}$ & $10^{-6}$ & $3 \times 10^{-6}$ & $10^{-5}$ & $3 \times 10^{-5}$ \\
\hline $\mathbf{0}$ & $\begin{array}{l}0.0247 \\
-\end{array}$ & $\begin{array}{l}\mathbf{0 . 0 5 2} \\
0.052\end{array}$ & $\begin{array}{l}\mathbf{0 . 2 3 4 8} \\
0.257\end{array}$ & $\begin{array}{l}\mathbf{0 . 7 8} \\
0.78\end{array}$ & $\begin{array}{l}1 \\
1\end{array}$ & $\begin{array}{l}\mathbf{1} \\
1\end{array}$ & $\begin{array}{l}1 \\
1\end{array}$ \\
\hline 1 & $\begin{array}{l}0 \\
-\end{array}$ & $\begin{array}{l}\mathbf{0} \\
0\end{array}$ & $\begin{array}{l}\mathbf{0} \\
0\end{array}$ & $\begin{array}{l}\mathbf{0 . 4 2 1 6} \\
0.43\end{array}$ & $\begin{array}{l}1 \\
1\end{array}$ & $\begin{array}{l}1 \\
1\end{array}$ & $\begin{array}{l}1 \\
1\end{array}$ \\
\hline 2 & $\begin{array}{l}\mathbf{0} \\
-\end{array}$ & $\begin{array}{l}\mathbf{0} \\
0\end{array}$ & $\begin{array}{l}\mathbf{0} \\
0\end{array}$ & $\begin{array}{l}\mathbf{0 . 0 4 7 2} \\
0.039\end{array}$ & $\begin{array}{l}\mathbf{0 . 9 8 8} \\
1\end{array}$ & $\begin{array}{l}\mathbf{1} \\
1\end{array}$ & $\begin{array}{l}1 \\
1\end{array}$ \\
\hline 3 & $\begin{array}{l}0 \\
-\end{array}$ & $\begin{array}{l}\mathbf{0} \\
0\end{array}$ & $\begin{array}{l}\mathbf{0} \\
0\end{array}$ & $\begin{array}{l}\mathbf{0} \\
0\end{array}$ & $\begin{array}{l}\mathbf{0 . 9 5 0 8} \\
0.96\end{array}$ & $\begin{array}{l}1 \\
1\end{array}$ & $\begin{array}{l}1 \\
1\end{array}$ \\
\hline
\end{tabular}

In the case of steady diffusion, the flux of ions towards the particle is (Fuchs, 1963):

$$
I=\frac{4 \pi a D n_{0}}{\frac{4 D a}{\alpha \bar{c} \delta^{2}} \exp \left[\frac{\varphi(\delta)}{k T}\right]+\Psi}
$$

where

$\Psi=\int_{0}^{\alpha / \delta} \exp \left[\frac{\varphi(a / x)}{k T}\right] d x$

The quantity $I / n_{0}$ is the combination coefficients $\eta_{i j}$ for aerosol particles and gaseous ions. $\mathrm{i}$ is the number of the elementary charges on the particles before the combination, and $j=i \pm 1$ after it. The stationary ratios $\mathrm{N}_{\mathrm{i}} / 2 \mathrm{~N}_{0}$, where $\mathrm{N}_{\mathrm{i}}$ is the total number of particles 
with i positive or negative charges, $\mathrm{N}_{0}$ the number of uncharged particles can be calculated by (Fuchs, 1963):

$N_{n} / 2 N_{0}=\left(\eta_{01} / \eta_{10}\right)\left(\eta_{12} / \eta_{21}\right) \ldots$

Table B.3 Comparison of the stationary charge distribution on particles calculated in this work (top) with Fuch's calculation, $\left(D=0.04 \mathrm{~cm}^{2} / \mathrm{sec}, c=4.625 \times 10^{4} \mathrm{~cm} / \mathrm{sec}, \lambda=1.3 \times 10^{-6} \mathrm{~cm}\right)$

\begin{tabular}{|c|c|c|c|c|c|c|c|}
\hline $\mathrm{a}(\mathrm{cm})$ & $10^{-7}$ & $3 \times 10^{-7}$ & $10^{-6}$ & $3 \times 10^{-6}$ & $10^{-5}$ & $3 \times 10^{-5}$ & $10^{-4}$ \\
\hline $\mathrm{N}_{1} / \mathbf{2} \mathrm{N}_{0}$ & $\begin{array}{l}\mathbf{0 . 0 0 6 6} \\
0.0056\end{array}$ & $\begin{array}{l}\mathbf{0 . 0 3 0 8} \\
0.029\end{array}$ & $\begin{array}{l}\mathbf{0 . 1 5 9 7} \\
0.159\end{array}$ & $\begin{array}{l}\mathbf{0 . 4 5 4 3} \\
0.45\end{array}$ & $\begin{array}{l}\mathbf{0 . 7 7 6 5} \\
0.77\end{array}$ & $\begin{array}{l}\mathbf{0 . 9 1 5 7} \\
0.91\end{array}$ & $\begin{array}{l}\mathbf{0 . 9 7 3 0} \\
0.97\end{array}$ \\
\hline $\mathrm{N}_{2} / 2 \mathrm{~N}_{0}$ & $\begin{array}{l}\mathbf{0} \\
0\end{array}$ & $\begin{array}{l}\mathbf{0} \\
0\end{array}$ & $\begin{array}{l}\mathbf{0 . 5 6 7 8} \\
0.56\end{array}$ & $\begin{array}{l}\mathbf{0 . 0 4 2 6} \\
0.043\end{array}$ & $\begin{array}{l}\mathbf{0 . 3 6 3 2} \\
0.36\end{array}$ & $\begin{array}{l}\mathbf{0 . 7 0 3 1} \\
0.71\end{array}$ & $\begin{array}{l}\mathbf{0 . 8 9 6 2} \\
0.89\end{array}$ \\
\hline $\mathbf{N}_{3} / 2 \mathbf{N}_{0}$ & $\begin{array}{l}\mathbf{0} \\
0\end{array}$ & $\begin{array}{l}\mathbf{0} \\
0\end{array}$ & $\begin{array}{l}\mathbf{0 . 1 0 7 9} \times 10^{-7} \\
\sim 0\end{array}$ & $\begin{array}{l}\mathbf{0 . 8 1 5 7} \times \mathbf{1 0}^{-3} \\
0.81 \times 10^{-3}\end{array}$ & $\begin{array}{l}\mathbf{0 . 1 0 2 2} \\
0.102\end{array}$ & $\begin{array}{l}\mathbf{0 . 4 5 2 7} \\
0.46\end{array}$ & $\begin{array}{l}\mathbf{0 . 7 8 1 4} \\
0.78\end{array}$ \\
\hline $\mathrm{N}_{4} / 2 \mathrm{~N}_{0}$ & $\begin{array}{l}\mathbf{0} \\
0\end{array}$ & $\begin{array}{l}\mathbf{0} \\
0\end{array}$ & $\begin{array}{l}\mathbf{0} \\
0\end{array}$ & $\begin{array}{l}\mathbf{0 . 3 1 0 6} \times 10^{-5} \\
\sim 0\end{array}$ & $\begin{array}{l}\mathbf{0 . 0 1 7 2} \\
0.021\end{array}$ & $\begin{array}{l}\mathbf{0 . 2 4 4 4} \\
0.25\end{array}$ & $\begin{array}{l}\mathbf{0 . 6 4 5 0} \\
0.64\end{array}$ \\
\hline
\end{tabular}

When the properties of positive ion are different from those of negative ion, the basic equations for bipolar diffusion charging are (Adachi, 1985):

$$
\begin{array}{ll}
\frac{d n_{0}}{d t}=\eta_{+1}^{-} n_{+1} N^{-}-\eta_{0}^{-} n_{0} N^{-}+\eta_{-1}^{+} n_{-1} N^{+}-\eta_{0}^{+} n_{0} N^{+} & \\
\frac{d n_{p}}{d t}=\eta_{p+1}^{-} n_{p+1} N^{-}-\eta_{p}^{-} n_{p} N^{-}+\eta_{p-1}^{+} n_{p-1} N^{+}-\eta_{p}^{+} n_{p} N^{+} & |p| \geq 1
\end{array}
$$

where $\mathrm{n}_{0}, \mathrm{n}_{\mathrm{p}}$ are number concentrations of particles carrying zero and $\mathrm{p}$ elementary charge unit and $\mathrm{N}$ is ion concentration. First and third terms are production terms and second and fourth terms are dissipation terms.

By assuming that the combination probability of particles having large $|p|$ and the number concentration of particles having $|p|_{+1}$ charges are zero if the ion and the particle have the same polarity, and if the number concentrations of positive and negative ions are the same, the basic equations are simplified in the stationary state as (Adachi, 1985):

$$
\begin{array}{ll}
n_{p} / n_{p-1}=\eta_{p-1}^{+} / \eta_{p}^{-} & p \geq+1 \\
n_{p} / n_{p+1}=\eta_{p+1}^{-} / \eta_{p}^{+} & p \leq-1
\end{array}
$$

and the number ratio of charged particles to total particles is (Adachi, 1985):

$$
\begin{array}{ll}
n_{p} / n_{T}=\prod_{p=+1}^{+\infty}\left(\eta_{p-1}^{+} / \eta_{p}^{-}\right) / \sum & p \geq+1 \\
n_{p} / n_{T}=\prod_{p=-1}^{-\infty}\left(\eta_{p+1}^{-} / \eta_{p}^{+}\right) / \sum & p \leq-1 \\
n_{0} / n_{T}=1 / \sum &
\end{array}
$$




$$
\sum=\sum_{p=+1}^{+\infty}\left\{\prod_{p=+1}^{+\infty}\left(\eta_{p-1}^{+} / \eta_{p}^{-}\right)\right\}+\sum_{p=-1}^{-\infty}\left\{\prod_{p=-1}^{-\infty}\left(\eta_{p+1}^{-} / \eta_{p}^{+}\right)\right\}+1
$$

$\mathrm{n}_{\mathrm{T}}$ is the number concentration of total particles.

Values of the mean thermal velocity of ions $\left(c_{i o n}\right)$, diffusion coefficient of ions $\left(D_{\text {ion }}\right)$, and mean free path of ions $\left(\lambda_{\text {ion }}\right)$, can be calculated by the following formulas (Adachi, 1985):

$$
\begin{aligned}
& D_{\text {ion }}=k T Z_{i o n} / e \\
& c_{i o n}=\sqrt{8 k T / \pi\left(M_{i o n} / N_{a}\right)} \\
& \lambda_{\text {ion }}=1.329 \frac{Z_{\text {ion }}}{e} \sqrt{\frac{k T M_{\text {ion }} M_{\text {gas }}}{\left(M_{\text {ion }}+M_{\text {gas }}\right) N_{a}}}
\end{aligned}
$$

where e is elementary electrical charge $\left(1.6021 \times 10^{-19} \mathrm{C}\right), \mathrm{N}_{\mathrm{a}}$ Avagadro number $\left(6.0238 \times 10^{23}\right.$ $\left.\mathrm{mol}^{-1}\right), \mathrm{k}$ is Boltzmann's constant $\left(1.3806 \times 10^{-23} \mathrm{~J} / \mathrm{K}\right)$ and $\mathrm{Z}$ electrical mobility of ion $\left(\mathrm{m}^{2} /\right.$ V.S $)$.

Applying positive and negative ion parameters (mobility and mass) for air, argon, and nitrogen, given by Wiedensohler (1991), in the above-mentioned formulas, Table B.4 was calculated.

Table B.4 Calculated positive and negative ions properties in different gases

\begin{tabular}{|l|l|l|l|}
\hline & Ar & $\mathrm{N}_{2}$ & Air \\
\hline $\mathbf{D}^{+}\left(\mathbf{c m}^{2} / \mathbf{s e c}\right)$ & $3.54 \times 10^{-2}$ & $5.06 \times 10^{-2}$ & $3.55 \times 10^{-2}$ \\
$\mathbf{D}^{-}$ & $4.3 \times 10^{-2}$ & $5.44 \times 10^{-2}$ & $4.05 \times 10^{-2}$ \\
\hline $\mathbf{c}^{+}(\mathbf{c m} / \mathbf{s e c})$ & $2.37 \times 10^{4}$ & $2.37 \times 10^{4}$ & $2.1 \times 10^{4}$ \\
$\mathbf{c}^{-}$ & $3.22 \times 10^{4}$ & $3.22 \times 10^{4}$ & $2.48 \times 10^{4}$ \\
\hline$\lambda^{+}(\mathbf{c m})$ & $1.44 \times 10^{-6}$ & $2.06 \times 10^{-6}$ & $1.47 \times 10^{-6}$ \\
$\lambda^{-}$ & $1.61 \times 10^{-6}$ & $2.04 \times 10^{-6}$ & $1.63 \times 10^{-6}$ \\
\hline
\end{tabular}

Finally a MATLAB code was developed to calculate the stationary state charge distribution on particles, under various atmospheres using parameters given in Table B.4 (Figures B.5, B.6).

The Fuchs model must be solved numerically. The distribution obtained from Fuch's model was approximated by Wiedensohler (1988), via an empirical expression which allows a quick solution of the bipolar charge distribution in the size range of 1-1000 nm.

$1 \mathrm{~nm} \leq D_{p} \leq 1000 \mathrm{~nm}$ for $N=-1,0,1$

$20 \mathrm{~nm} \leq D_{p} \leq 1000 \mathrm{~nm}$ for $N=-2,2$ 


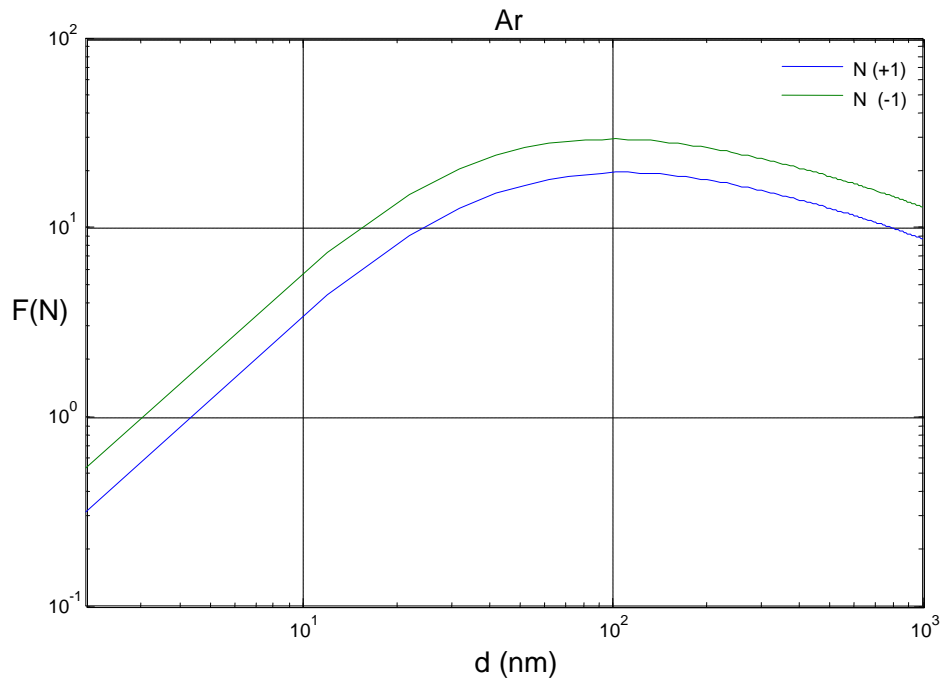

Figure B.5 Fraction of particles carrying one charge exposed to bipolar ions in Argon

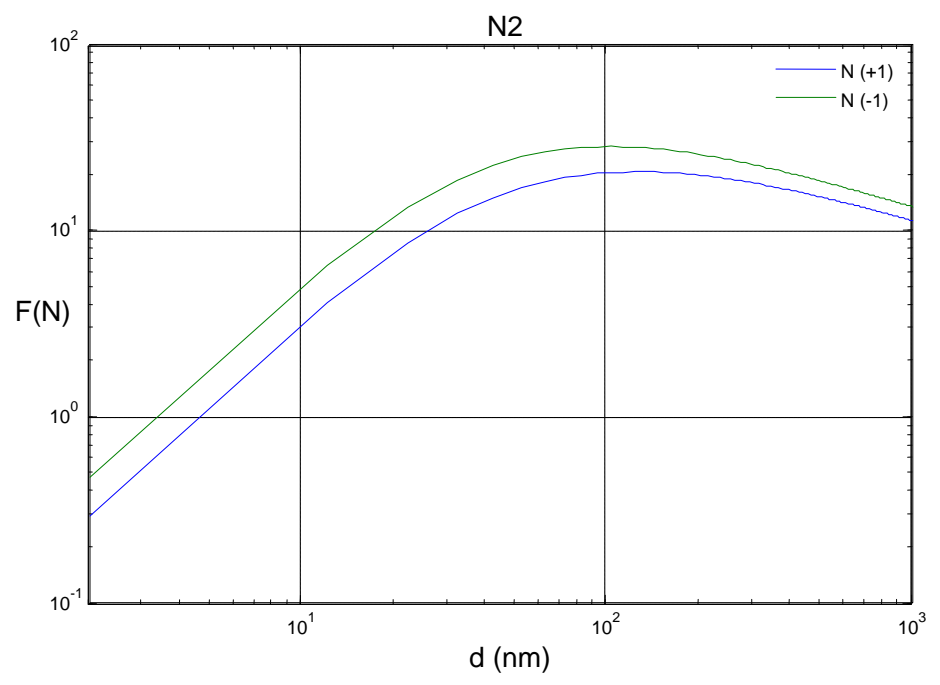

Figure B.6 Fraction of particles carrying one charge exposed to bipolar ions in Nitrogen

Wiedensohler et al. (1986) used least square regression analysis to determine the approximation coefficients:

$$
f(N)=10^{\left[\sum_{i=0}^{5} a_{i}(N)\left(\log \frac{D_{p}}{n m}\right)^{i}\right]}
$$

Table B.5 The approximation coefficients (Wiedensohler, et al., 1986)

\begin{tabular}{|l|l|l|l|l|l|}
\hline $\mathrm{a}_{\mathrm{i}}(\mathbf{N})$ & $\mathbf{N}=\mathbf{- 2}$ & $\mathbf{N}=\mathbf{- 1}$ & $\mathbf{N}=\mathbf{0}$ & $\mathbf{N}=\mathbf{1}$ & $\mathbf{N}=\mathbf{2}$ \\
\hline $\mathbf{a}_{\mathbf{0}}$ & -26.3328 & -2.3197 & -.0003 & -0.2 .3484 & -44.4756 \\
\hline $\mathbf{a}_{\mathbf{1}}$ & 35.9044 & 0.6175 & -0.1014 & 0.6044 & 79.3772 \\
\hline $\mathbf{a}_{\mathbf{2}}$ & -21.4608 & 0.6201 & 0.3073 & 0.4800 & -0.62 .8900 \\
\hline $\mathbf{a}_{3}$ & 7.0867 & -0.1105 & -0.3372 & 0.0013 & 26.4492 \\
\hline $\mathbf{a}_{4}$ & -1.3088 & -0.1260 & 0.1023 & -0.1553 & -.5 .7480 \\
\hline $\mathbf{a}_{\mathbf{5}}$ & 0.1051 & 0.0297 & -0.0105 & 0.0320 & 0.5049 \\
\hline
\end{tabular}


Figure B.7 is obtained using equation (B.34) and Table B.5.

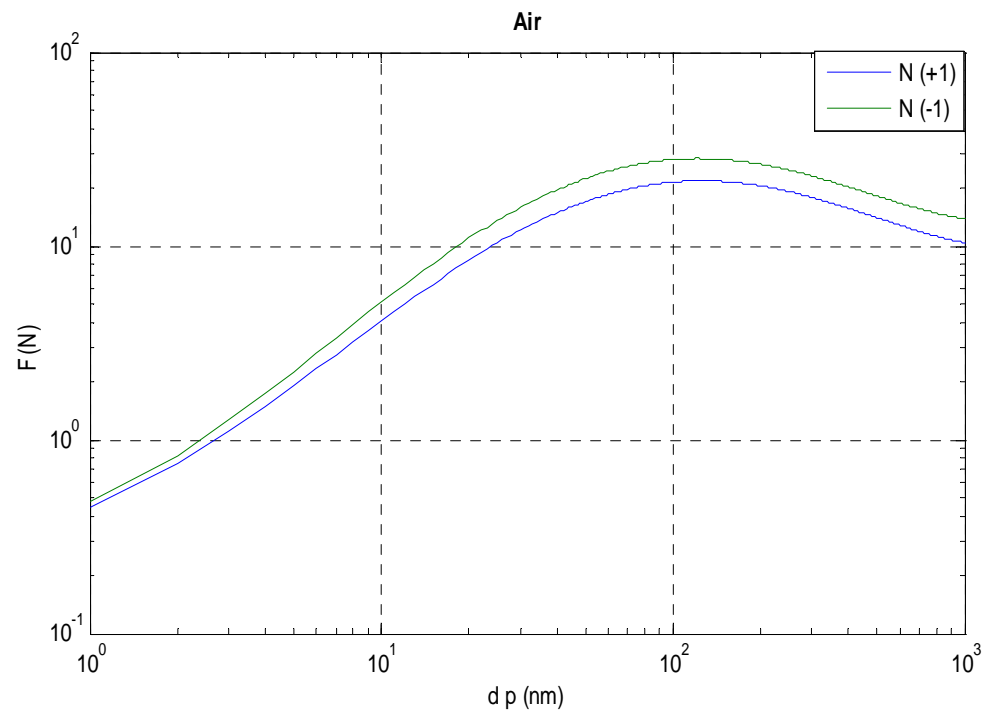

Figure B.7 Fraction of particles carrying one charge in equilibrium with bipolar ions in air

Assuming that the concentrations for positive and negative ions are equal, for the fraction of particles carrying more charges, following equation derived by Gunn (1956) can be used:

$f(N)=\frac{e}{\sqrt{4 \pi^{2} \varepsilon_{0} D_{p} k T}} \exp \frac{-\left[N-\frac{2 \pi \varepsilon_{0} D_{p} k T}{e^{2}} \ln \left(\frac{Z_{i+}}{Z_{i-}}\right)\right]^{2}}{2 \frac{2 \pi \varepsilon_{0} D_{p} k T}{e^{2}}}$

where:

e elementary charge $\left(1.60217733 \times 10^{-19} \mathrm{C}\right)$

$\varepsilon_{0} \quad$ dielectric constant $\left(8.854187817 \times 10^{-12} \mathrm{~F} / \mathrm{m}\right.$ for air $)$

$\mathrm{D}_{\mathrm{p}} \quad$ particle diameter $(\mathrm{m})$

$\mathrm{k} \quad$ Boltzmann's constant $\left(1.380658 \times 10^{-23} \mathrm{~J} / \mathrm{K}\right.$ for air $)$

$\mathrm{T} \quad$ temperature $(\mathrm{K})$

$\mathrm{N} \quad$ number of elementary charge units

$\mathrm{Z}_{\mathrm{i}+} / \mathrm{Z}_{\mathrm{i}-} \quad$ ion mobility ratio (0.875 Wiedensohler, 1986) 


\section{B.4 Aerosol Electrometer}

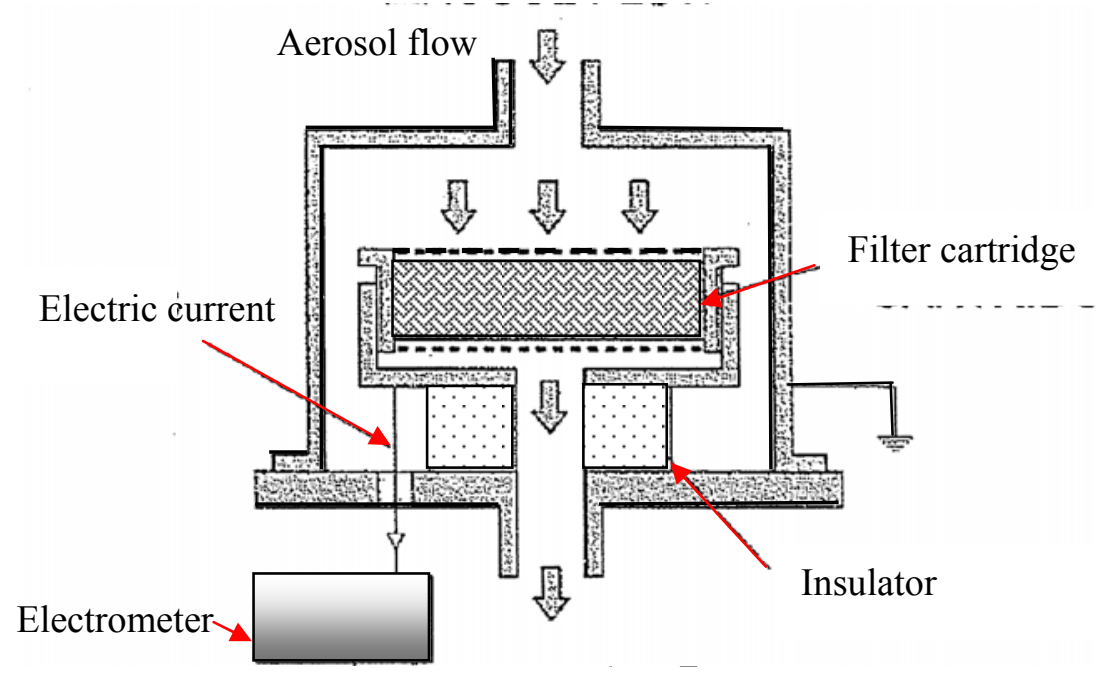

Figure B.8 Schematic of Aerosol Electrometer

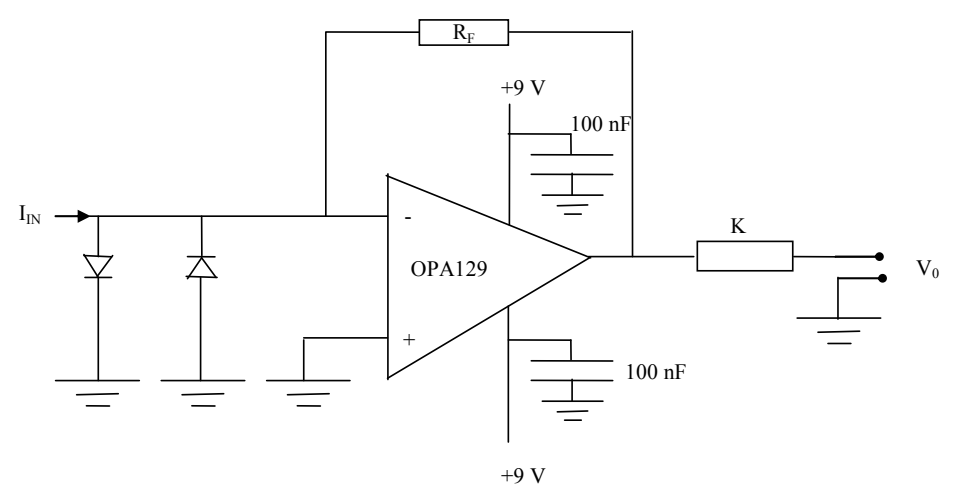

Figure B.9 Electric circuit of Aerosol Electrometer (Galdhar, 2004)

The Faraday cup aerosol electrometer consists of an electrometer and a filter inside a Faraday cage mounted on an insulator. Charged particles collected by the filter, generate an electric current which is measured by the electrometer. The electric circuit of the electrometer is schematized in Figure B.9. OPA129 is an ultra low bias current monolithic operational amplifier. The relationship between the input current and the output voltage and the particle number concentration is as follows (Gahldbar, 2004):

$I=\frac{V}{R}=n e Q N$

I electric current (A)

$\mathrm{R}$ resistance (typically $100 \mathrm{G} \Omega$ )

e electron charge $\left(1.6 \times 10^{-19} \mathrm{C}\right)$

$\mathrm{N}$ particle concentration $\left(\# \mathrm{~m}^{-3}\right)$

$\mathrm{V}$ voltage $(\mathrm{V})$

$\mathrm{n}$ number of elementary charge

$\mathrm{Q}$ gas flow rate $\left(\mathrm{m}^{3} \mathrm{~s}^{-1}\right)$

It is typically used to measure particles of unipolar charge. The electrometer amplifier is sensitive to external conditions especially temperature. Thus the amplifier zero value may drift over period of time. It can be used under vacuum condition and there is no theoritical limitation on the upper particle concentration measurement. 


\section{B.5 Electrostatic Precipitator}

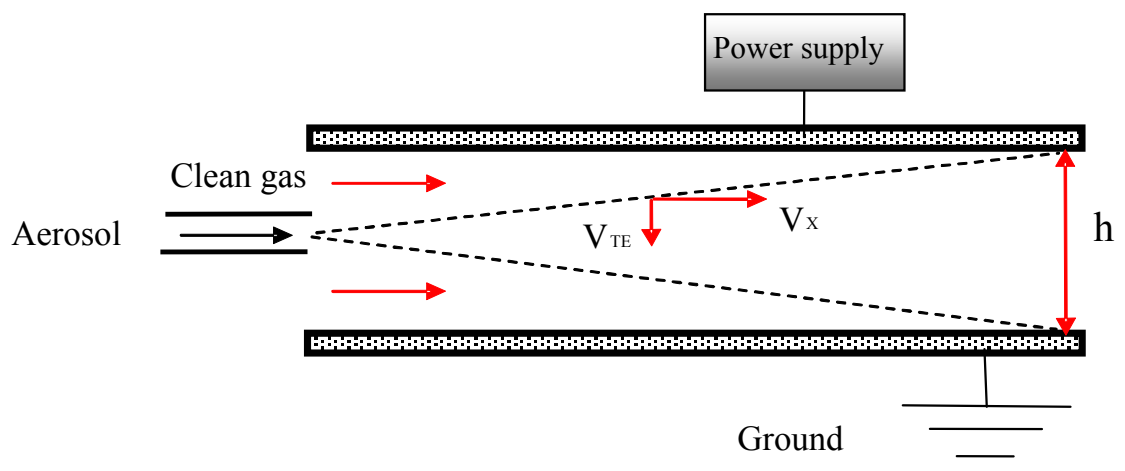

Figure B.10 Schematic of electrostatic precipitator

An electrostatic precipitator (ESP) is a particulate collection device that removes particles from a flowing gas using the force of an induced electrostatic field.

Particles are introduced along the centerline between two oppositely charged parallel plates. For a given voltage, all particles with electrical mobility greater than a certain value will migrate to the plates and get trapped. The particles with lower mobility and uncharged particles pass through unaffected (Hinds, 1999).

$$
\begin{array}{ll}
V_{T E}=\frac{n e E C_{c}}{3 \pi \eta d_{p}} & \text { for } \operatorname{Re}<1 \\
X=\frac{V_{X} h}{V_{T E}} &
\end{array}
$$

where :

n number of elementary charge on particles

e charge of electron $\left(1.6 \times 10^{-19} \mathrm{C}\right)$

E electric field strength $(\mathrm{V} / \mathrm{m})$

$\mathrm{C}_{\mathrm{c}} \quad$ slip correction factor

$\eta \quad$ viscosity (Pa.s)

$\mathrm{d}_{\mathrm{p}} \quad$ particle diameter $(\mathrm{m})$

$\mathrm{V}_{\mathrm{x}}$ velocity in $\mathrm{X}$ direction $(\mathrm{m} / \mathrm{s})$

$\mathrm{V}_{\mathrm{T}}$ terminal velocity $(\mathrm{m} / \mathrm{s})$

$\mathrm{h} \quad$ distance between the plates $(\mathrm{m})$ 


\section{B.6 Impactor}

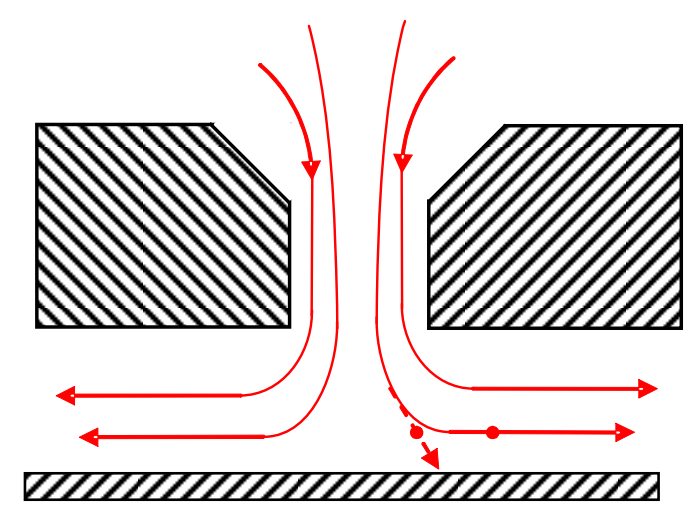

Figure B.11 Schematic of cross section of an impactor

Impactors are used for the collection and measurement of aerosol particles. In imactors the aerosol passes through a nozzle and the output flow is directed against a flat plate which deflects the stream to form a $90^{\circ}$ bend in the streamlines. Particles with inertia exceeding a certain value, unable of following the streamlines, collide on the impaction plate. Thus particles are separated into two size ranges; particles larger than a certain aerodynamic diameter are removed, and those with smaller size remain in the flow and get through the impactor. The parameter governing the collection efficiency is the Stokes number which for $50 \%$ collection efficiency in circular jets is 0.24 (Hinds, 1999). The following equation relates the cutoff diameter $\mathrm{d}_{50}$ (particle size having $50 \%$ collection efficiency), the jet diameter $D_{j}$, gas flow rate $Q$, slip factor $C_{c}, S_{k}$, density of particle $\rho_{p}$, and the flow viscosity $\eta$ (Hinds, 1999):

$d_{50} \sqrt{C_{c}}=\left[\frac{9 \pi \eta D_{j}^{3}\left(S t k_{50}\right)}{4 \rho_{p} Q}\right]^{1 / 2}$

Because $\mathrm{C}_{\mathrm{c}}$ is a function of $\mathrm{d}_{50}$, the following empirical equation can be used to approximate $\mathrm{d}_{50}$ (Hinds, 1999):

$d_{50}=d_{50} \sqrt{C_{c}}-0.078$

for $d_{50}$ in $\mu \mathrm{m}$. This equation is accurate for $d_{50}>0.2 \mu \mathrm{m}$ and pressure from $0.9-1$ bar. For obtaining a sharp cutoff, the design criteria are:

- $\operatorname{Re}$ (in the nozzle throat) between 500-3000

- The ratio of the distance between the nozzle and the plate to the jet diameter, between 1-5 with the lower values preferred.

For a given cutoff size, the Re can be adjusted by applying multiple nozzles in parallel. The smallest cutoff sizes for conventional impactors are 0.2-0.3 $\mu \mathrm{m}$. For obtaining $0.05 \mu \mathrm{m}$ cutoff size, micro-orifice impactors with large numbers of chemically etched nozzles as small as 50 $\mu \mathrm{m}$ in diameter can be used. Another approach is the low-pressure (0.03-0.4 bar) impactor in which the slip correction factor is increased, leading to reduction the cutoff size (Hinds, 1999). Because of high jet velocities, particle bouncing can occur. 


\section{References}

Adachi M., Kousaka Y., Okuyama, Unipolar and Bipolar Diffusion Charging of Ultrafine Aerosol Particles, Journal of Aerosol Science, Volume 16, Number 2, 1985, pp. 109-123

Fissan H., D. Hummes, F. Stratmann, P. Buscher, S. Neumann, Experimental Comparison of Four Differential Mobility Analyzer for Nanometer Aerosol Measurements, Aerosol Science and Thecnology, Volume 24, 1996, pp. 1-13

Fuchs N. A., On the Stationary Charge Distribution on Aerosol Particles in a Bipolar Ionic Atmosphere, Pure and Applied Geophysics , 1963, Volume 56, Number 1, pp. 185-193

Gahldhar A., Design of an Electrical Aerosol Sensor, Master thesis, TUDelft, 2004

Gunn R., R. H. Woessner, Measurements Related to the Fundamental Processes of Aerosol Electrification, Journal of Colloid Science, Volume 11, 1956, pp. 254-259

Hinds William C., Aerosol Technology, Properties, Behaviors,, and Measurements of Airborne Particles, John Wiley\&Sons, 1999

Hussin A., Scheibel, K. H. Becker, J. Porstendorfer, Bipolar Diffusion Charging of Aerosol Particles-I: Experimental Results within the Diameter Range 4-30 nm, Journal of Aerosol Science, Volume 14, Number 5, 1983, pp. 671-677

Knutson E. O., Whitby K. T., Accurate Measurement of Aerosol Electric Mobility Moments, Journal of Aerosol Science, Volume 6, 1975, pp. 453-460

Knutson E. O., Whitby K. T., Aerosol Classification by Electric Mobility: Apparatus, Theory, and Applications, Journal of Aerosol Science, Volume 6, 1975, pp. 443-451

Wiedensohler A., H. J. Fissan, Aerosol Charging in High Purity Gases, Journal of Aerosol Science, Volume 19, Number 7, 1988, pp. 867-870

Wiedensohler A., H. J. Fissan, Bipolar Charge Distributions of Aerosol Particles in High-Purity Argon and Nitrogen, Aerosol Science and Technology, Volume 14, 1991, pp. 358-364

Wiedensohler A., Lutkemeier, Feldpausch, C. Helsper, Investigation of the Bipolar Charge Distribution at Various Gas Conditions, Journal of Aerosol Science, Volume 17, Number 3, 1986, pp. 413-416

Wiedensohler A., Thecnical Note An Approximation of the Bipolar Charge Distribution for Particles in the Submicron Size Range, Journal of Aerosol Science, Volume 19, Number 3, 1988, pp. 387-389 


\section{C.1 Formation and Growth of Nanoparticles}

Particle formation in the gas phase usually involves with rapid quenching of supersaturated vapor in non-equilibrium conditions. When a nucleus is formed, it should reach a critical size in order to have chance for further growth, otherwise it evaporates. Due to the curved surface of nuclei, attractive forces between surface molecules are modified and the required partial pressure to maintain mass equilibrium is higher than the saturation vapor pressure for a plane surface. Kelvin equation (Hinds, 1999) gives the relationship between the critical particle size (no growth no evaporation) and degree of supersaturation. Higher supersaturation results in the smaller critical nucleus size. The nucleation and growth of particles is determined primarily by the local cooling rate, the residence time distribution, and the number density in the nucleation and growth zone.

Particle growth in gas phase include two major processes: the absorption growth process where particle nuclei grow up with absorption of atoms, and the coalescence growth process where particles grow up by collision of particle nuclei or cluster (Sugimoto, 2000).

The combination of these processes leads to two well-known distributions, the normal like distribution and the lognormal distribution, depending on the growth conditions.

In general, the lognormal distribution is found and results from a coalescence growth of the clusters. However, the absorption mechanism is effective in the very earliest stages of the particle growth and for the normal distribution the number frequency function is given by (Hinds, 1999):

$$
\begin{aligned}
d f & =\frac{1}{\sigma \sqrt{2 \pi}} \exp \left(-\frac{\left(d_{p}-\bar{d}_{p}\right)^{2}}{2 \sigma^{2}}\right) d d_{p} \\
\sigma & =\left(\frac{\sum n_{i}\left(d_{i}-\bar{d}_{p}\right)^{2}}{N-1}\right)^{1 / 2} \\
\bar{d}_{p} & =\frac{\sum d}{N}
\end{aligned}
$$

Following the very early step of the growth, many nuclei exist in the growth zone and the major step for the particle growth is collision of particle nuclei or clusters. A lognormal distribution function is defined by (Hinds, 1999):

$$
\begin{aligned}
& d f=\frac{1}{\sqrt{2 \pi} d_{p} \ln \sigma_{g}} \exp \left(-\frac{\left(\ln d_{p}-\ln C M D\right)^{2}}{2\left(\ln \sigma_{g}\right)^{2}}\right) d d_{p} \\
& \ln \sigma_{g}=\left(\frac{\sum n_{i}\left(\ln d_{i}-\ln d_{g}\right)^{2}}{N-1}\right)^{1 / 2} \\
& \ln d_{g}=\frac{\sum n_{i} \ln d_{i}}{N}
\end{aligned}
$$

In aerosol, particles collide with each other due to relative motion between them and adhere to form larger structures. If the relative motion between the particles is due to Brownian motion, the process is thermal coagulation which depends on temperature, primary particle size, agglomerate size and structure, and the concentration squared; hence it is of more significance for fine particles of concentrations above $10^{6}-10^{7} \mathrm{~cm}^{-3}$ (Borra, 2006). 
Agglomeration process can be enhanced by attractive electrical forces between the particles. Although charged particles with opposite signs have an enhanced probability of collision, this is offset by reduced collisions between particles with the same sign. Thus for aerosol with Boltzmann equilibrium charge distribution, the net result is a negligible change in the agglomeration. However for aerosol with strong bipolar charge distribution, the agglomeration can significantly be increased (Hinds, 1999). Gas borne particles with unipolar charge distribution are repelled from one another slowing the rate of coagulation (Hinds, 1999).

Agglomerates are composed of primary particles forming irregular structures. The behavior of these agglomerates differs from sphere with the same mass. Agglomerates structures usually obey a power-law relationship between radius and mass (Wu et al., 1993):

$$
N=A\left(\frac{r}{r_{0}}\right)^{D_{f}}
$$

$\mathrm{D}_{\mathrm{f}}$ is fractal dimension, varying between 1 (line) to 3 (sphere). Cluster-cluster agglomerations have shown that Brownian movement dominated agglomeration processes tend to produce agglomerates with fairly low fractal dimensions $1.6<\mathrm{D}_{f}<2.2$ (Lehtinen et al., 1996). $\mathrm{N}$ is the number of primary particles with uniform size of $\left(\mathrm{r}_{0}\right)$ and mass of $(\mathrm{m}),(\mathrm{r})$ is a characteristic radius for example radius of gyration (Wu et al., 1993):

$$
r_{g}=\sqrt{\frac{\sum_{i} m r_{i}^{2}}{\sum_{i} m}}
$$

$r_{i}$ is the distance of the $i^{\text {th }}$ primary particle from the center of mass. The dimensionless proportionality constant (A) depends on the characteristic radius definition, fractal dimension, and the agglomeration mechanism. It indicates the packing of the primary particles and is around unity (Table 3.1).

Table C.1 Values of A based on radius of gyration (Wu et al., 1993)

\begin{tabular}{|l|l|l|l|}
\hline Agglomerate type & $\mathrm{N}$ & $\mathrm{D}_{\mathrm{f}}$ & $\mathrm{A}$ \\
\hline Cluster-cluster $(\mathrm{F})$ & $5-200$ & 1.82 & 1.37 \\
\hline Cluster-cluster (F) & $10-200$ & 1.84 & 1.30 \\
\hline Cluster-cluster (F) & $10-500$ & 1.91 & 1.30 \\
\hline \multicolumn{4}{|c|}{$(F)$ denotes free molecular regime }
\end{tabular}

For a sufficiently wide size distribution (GSD $>1.5$ ), adhesion of small particles to the large ones, make the size distribution narrower with time. On the other hand, agglomeration of monodisperse particles gradually leads to a polydisperse aerosol with wider size distribution. These competing mechanisms in agglomeration lead to an asymptotic, stable size distribution called self-preserving size distribution which is almost lognormal with a GSD of 1.32-1.36 for the continuum region (Hinds, 1999). In free molecular regime, Vermury et al. (1995) obtained the number geometric standard deviation for different fractal dimensions, ranging from 1.462 for $D_{f}=3$ and increasing to 1.61 for $D_{f}=2$. Thus in the free molecular regime, the self-preserving size distributions broaden as the fractal dimension decreases. A quantitative criterion for the time to achieve the self-preserving size spectrum is derived by Lai et al. (1972). 


\section{C.2 Evaporation-Condensation in Multi-component Systems}

In the process of evaporation into vacuum, according to Langmuir equation for a vapor with energies following a Boltzmann distribution, the number of atoms striking a surface per unit area per unit time is (Powell, 2005):

$$
J=\frac{P}{\sqrt{2 \pi M R T}}
$$

where $\mathrm{M}$ is the molecular weight of the vapor. At equilibrium, the flux of evaporating atoms leaving the surface equals that arriving at the surface, so the evaporation flux is given by replacing $\mathrm{P}$ with $\mathrm{P}_{\mathrm{v}}$ (vapor pressure).

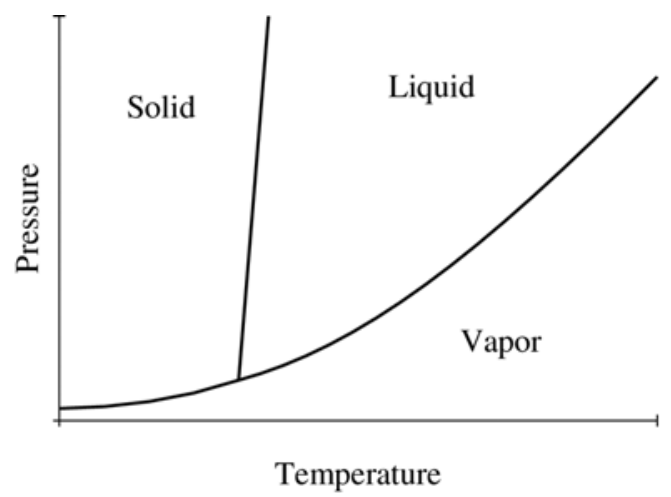

Figure C.1 Schematic phase diagram for a typical metal (Powell, 2005)

For a multi-component liquid or solid, the vapor pressure of each component is its activity (a) times the vapor pressure of the pure substance $\left(P_{v}=a \bar{P}_{v}\right)$.

For a dilute solution (liquid or solid) of solute B in solvent A, evaporating into a vacuum, the evaporation ratio is (Powell, 2005):

$$
E R_{B}=\frac{J_{B} X_{A}}{X_{B} J_{A}}=\frac{P_{v B}}{X_{B} \sqrt{M_{B}}} \frac{X_{A} \sqrt{M_{A}}}{P_{v A}}
$$

where $\mathrm{X}_{\mathrm{i}}$ represents the mole fraction of species $\mathrm{i}$ in the liquid or solid, $\mathrm{P}_{\mathrm{vi}}$ its vapor pressure, and $\mathrm{M}_{\mathrm{i}}$ its molecular weight. Assuming Raoult's law, the vapor pressure is the product of the vapor pressure in its pure state and mole fraction in liquid or solid, so:

$$
E R_{B}=\frac{P_{v B}}{X_{B} \bar{P}_{v A}} \sqrt{\frac{M_{A}}{M_{B}}}
$$

where $\bar{P}_{v i}$ denotes the vapor pressure of pure species i. Now assuming Henrian behavior the activity coefficient is:

$$
P_{v B}=\gamma_{B} \bar{P}_{v B} X_{B}
$$

Thus the evaporation ratio, which relates compositions in the vapor and condensed phases, is (Powell, 2005): 
$E V_{B}=\gamma_{B} \frac{\bar{P}_{v B}}{\bar{P}_{v A}} \sqrt{\frac{M_{A}}{M_{B}}}$

The formation of multicomponent materials by condensation of the vapors of two or more elements or compounds requires very rapid cooling rates for simultaneous nucleation and condensation of all the vapor species. The gas must be cooled to well below the temperature that yields a supersaturation for all the species present in a time much shorter than that required for significant particle formation and growth for the material with the lowest vapor pressure (Kodas and Hampden-Smith, 1999).

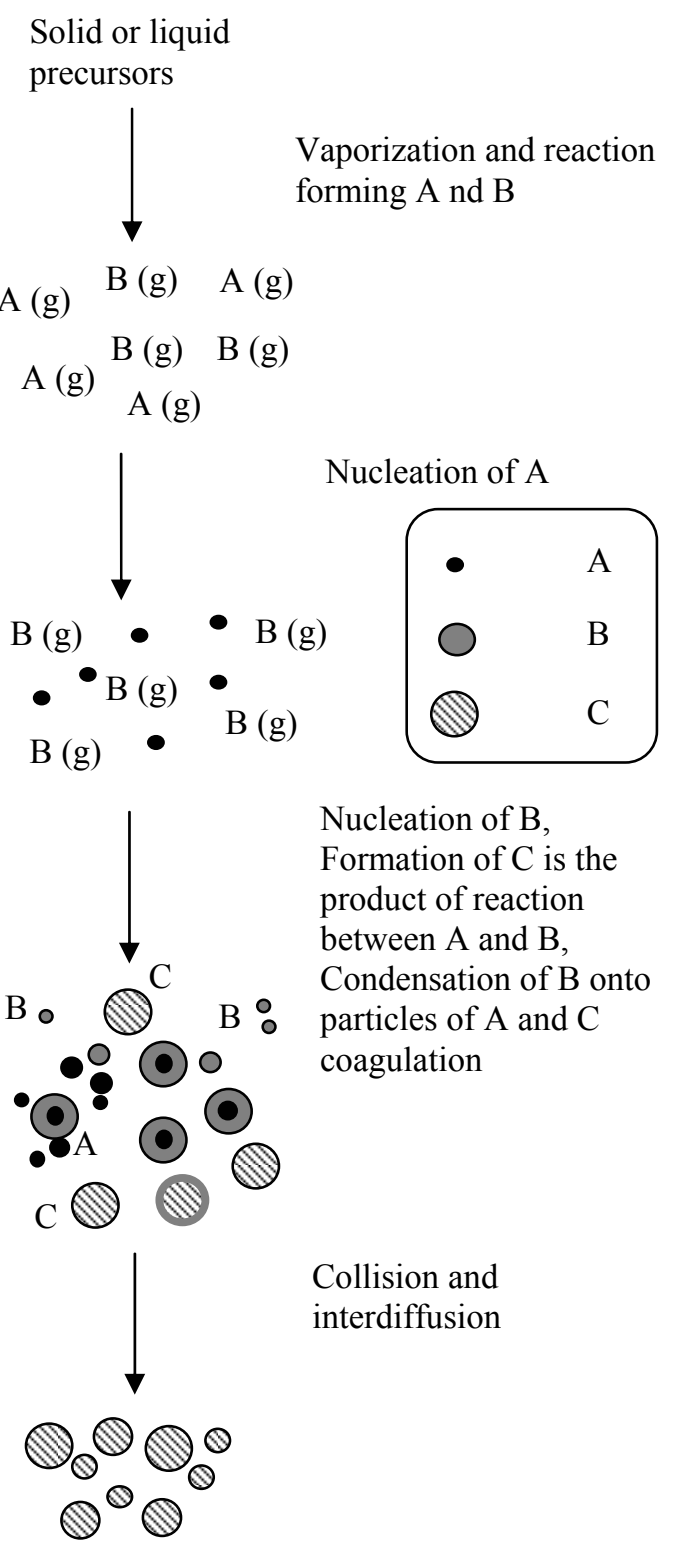

Figure C.2 Gas-to-particle conversion with two condensable vapors $A$ and $B$ (reproduced from Gurav et al., 1993)

Without sufficient, rapid cooling, the species with the lowest vapor pressure (A in Figure 4.2) forms particles when sufficiently high supersaturation is reached. As the temperature 
decreases, species $\mathrm{B}$, with the higher vapor pressure can condense onto the particles of the first species or may form new particles and result in core-shell structures or particles of each individual species. The ratio of $\mathrm{A}$ to $\mathrm{B}$ in the final product, $\mathrm{C}$, is determined by the collision of the different particles, followed by inter-diffusion (Kodas and Hampden-Smith, 1999).

\section{References}

Borra Jean- Pascal, Nucleation and Aerosol Processing in Atmospheric Pressure Electrical Discharges: Powders Production, Coatings and Filtration, J. Phys. D: Appl. Phys., Volume 39, 2006, R19-R54

Gurav A., T. Kodas, T. Pluym, Y. Xiong, Aerosol Processing of Materials, Journal of Aerosol Science and Technology, Volume 19, Number 4, 1993, pp. 411-452

Hinds William C. , Aerosol Technology, Properties, Behaviors, , and Measurements of Airborne Particles, John Wiley\&Sons, 1999

Kodas T. T., M. J. Hampden-Smith, Aerosol Processing of Materials, New York, Wiley-VCH, 1999

Lai F. S., S. K. Friedlander, J. Pich, G. M. Hidy, the Self-Preserving Particle Size Distribution for Brownian Coagulation in the Free-Molecule Regime, Journal of Colloid and Interface Science, Volume 39, Number 2, 1972, pp. 395-405

Lehtinen Kari E. J., Robert S. Windeler, Sheldon K. Friedlander, a Note on the Growth of Primary Particles in Agglomerate Structures by Coalescence, Journal of Colloid and Interface Science, Volume 182, 1996, pp. 606608

Powell A., J. Van Den Avyle, B. Damkroger, J. Szekely and U. Pal, Analysis of Multicomponent Evaporation in Electron Beam Melting and Refining of Titanium Alloy, Metall. Mater. Trans., volume 28B, Number 6, 1997 , pp. 1227-1239

Powell A., Multicomponent Evaporation Kinetics, 2005

Sugimoto Tadao, Fine Particles Synthesis, Characterization, and Mechanisms of Growth, Marcel Dekker, 2000

Vemury S., S. Pratsinis, Self Preserving Size Distributions of Agglomerates, Journal of Aerosol Science, Volume 26, 1995, pp. 175-185

Wu M. K., R. S. Windeler, C. K. R. Steiner, T. Börs, S. K. Friedlander, Controlled Synthesis of Nanosized Particles by Aerosol Processes, Aerosol Science and Technology, Volume 19, 1993, pp. 527-548 


\section{D.1 Hydrogen Storage}

With the recent advancements in fuel cell technology, hydrogen is now considered as one of the major energy carriers of the future. Hydrogen can be produced from natural gas reforming, coal gasification, or electrolysis. By using renewable energy sources like solar, wind or water power as a source of electricity in production of hydrogen via electrolysis of water, the need for hydrocarbons will be reduced. Besides, in conversion of chemical energy stored in $\mathrm{H}_{2}$ to electricity in a fuel cell, water is produced with zero emission of greenhouse gases.

Hydrogen can be transported as compressed gas or in liquid form, both of which call for very special care to take into account all the safety concerns. Moreover, liquefaction process consumes almost $30 \%$ of the hydrogen energy to compress and cool the hydrogen to $20 \mathrm{~K}$ (Koch, 2007). Pressurized gas storage is shown in Figure D.1 for steel and a hypothetical composite material (Züttel, 2003).

Another possibility is to store hydrogen in metal hydrides ${ }^{1}$. They have a higher hydrogen storage density $\left(6.5 \times 10^{22} \mathrm{H}\right.$ atoms $/ \mathrm{cm}^{3}$ for $\left.\mathrm{MgH}_{2}\right)$ than hydrogen gas $\left(0.99 \times 10^{22} \mathrm{H}\right.$ atoms $\left./ \mathrm{cm}^{3}\right)$ or liquid hydrogen $\left(4.2 \times 10^{22} \mathrm{H}\right.$ atoms $\left./ \mathrm{cm}^{3}\right)$ (Cooper et al., 2005). Because the dehydrogenation process is endothermic, metal hydrides could be considered to be inherently safe in the event of tank rupture or fire. A major drawback of hydrides is the increase in weight needed for storage.

Figure D.1 shows the volumetric and gravimetric hydrogen density of some hydrides (Züttel, 2003). $\mathrm{Mg}_{2} \mathrm{FeH}_{6}$ shows the highest volumetric hydrogen density of $150 \mathrm{kgm}^{-3}, \mathrm{BaReH}_{9}$ has the highest $\mathrm{H} / \mathrm{M}$ ratio of $4.5, \mathrm{LiBH}_{4}$ exhibits the highest gravimetric hydrogen density of 18 $\operatorname{mass} \%$.

Hydride formation comprises a number of steps in series ${ }^{2}$. The slowest step is the ratedetermining one ${ }^{3}$.

To study the properties of a hydride, pressure-composition isotherms can be most beneficial. A typical plot (Schlapbach, 2001) is shown in Figure D.2. As seen, by increasing the hydrogen pressure at isothermal conditions, the amount of hydrogen taken up by the metal $(\mathrm{H} / \mathrm{M})$ is slight. It corresponds to the formation of $\alpha$-phase which is solid solution of hydrogen in the metal. After saturation of $\alpha$-phase with hydrogen, the metal hydride starts to form ( $\beta$-phase) and increasing the pressure results in an increase in the amount of absorbed hydrogen. According to the Gibbs phase rule, when a new phase is formed one degree of freedom will be lost. The pressure at which the new phase is formed is called plateau pressure at which the two phase $(\alpha$ and $\beta$ ) co-exist.

\footnotetext{
${ }^{1}$ Binary hydrides (ionic hydrides: $s$ elements $+\mathrm{H}$, metallic hydrides: $\mathrm{d}$ elements $+\mathrm{H}$, covalent hydrides: $\mathrm{p}$ elements $+\mathrm{H}$ ), intermetallic hydrides $\left(\mathrm{AB}_{5}, \mathrm{AB}\right.$ and $\left.\mathrm{AB}_{2}\right)$, complex metal hydrides (transition metal complexes, metal complexes).

${ }^{2}$ Physisorption of hydrogen molecules-hydrogen molecules dissociation and chemisorption, surface penetration of hydrogen atoms-diffusion of hydrogen atoms through the hydride layer either by an interstitial or by a vacancy mechanism-formation of hydride at the metal-hydride interface (Fernández, 2002).

${ }^{3}$ For instance diffusion of hydrogen through $\mathrm{MgH}_{2}$ is reported to be more than a factor of 5000 slower than diffusion through $\mathrm{Mg}$ (Klassen et al., 2001). Thus the rate determining step in the desorption of hydrogen by magnesium and probably also in absorption process, is likely to be the hydrogen diffusion through the $\beta$ phase (Fernández, 2002).
} 


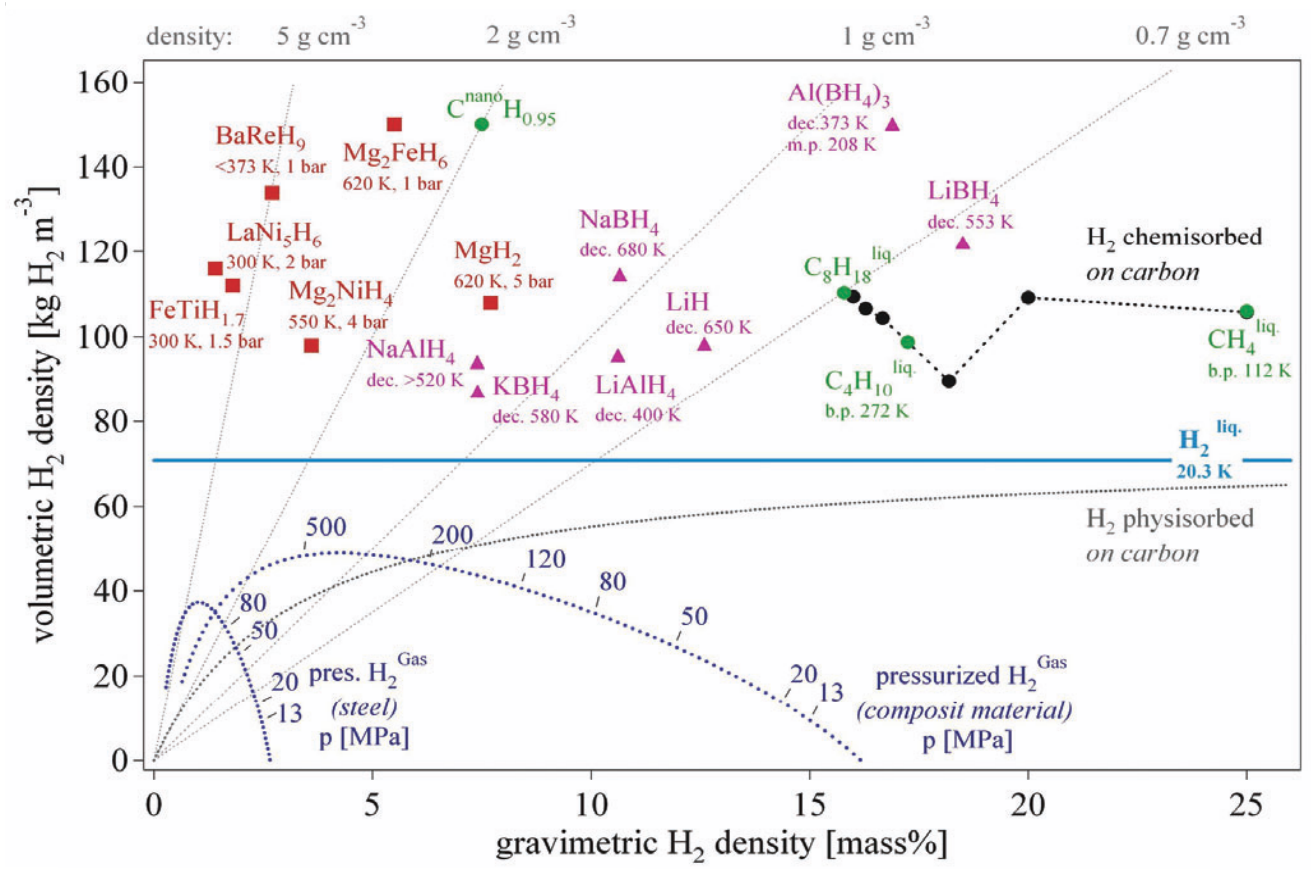

Figure D.1 Volumetric and gravimetric hydrogen density of some hydrides (by courtesy of Züttel, 2003)

After completion of hydride phase, increasing the pressure leads to the formation of solid solution of hydrogen in the hydride phase. In $\alpha$ phase the variation of pressure with concentration is in accordance with Sievert's law (Zuttel et al., 2000):

$\frac{x}{r-x}=k \sqrt{\frac{P}{P^{0}}}$

where $\mathrm{x}$ denotes the hydrogen to metal atom ratio, $\mathrm{r}$ the number of interstitial sites per metal atom, $\mathrm{k}$ a constant and $\mathrm{P}^{\circ}$ the reference pressure. In $\alpha+\beta$ region, the pressure does not ideally depend on the concentration. In the solid solution of hydrogen in the metal hydride phase, again Sievert's law correlates the pressure to the concentration.

Van't Hoff equation states that:

$$
\ln \left(\frac{P_{H_{2}}}{P^{0}}\right)=\frac{\Delta H_{f}}{R T}-\frac{\Delta S_{f}}{R}
$$

according to which, the plot of logarithm of the pressure plateau midpoint vs. reciprocal of the absolute temperature would be a straight line so that $\Delta H_{f}$ and $\Delta S_{f}$ can be derived from the slope and the intercept. At standard conditions $\left(\mathrm{P}_{\mathrm{H} 2}=\mathrm{P}^{\circ}\right)$, Van't Hoff equation reduces to:

$\Delta H_{f}=\Delta S_{f} T$

In most cases, the entropy change is mainly given by the loss of standard entropy of the hydrogen gas as it enters the metal lattice. Therefore, the entropy term does not depend significantly on the nature of metal and the $\Delta \mathrm{S}_{f}$ term could be considered constant, as $\Delta S_{f}=-$ $130 \mathrm{~J} / \mathrm{mol} \mathrm{K}$, (Andreasen, 2004). Thus for desorption of hydrogen at $100^{\circ} \mathrm{C}, \Delta H_{f}^{\circ}$ would be 
around $-48 \mathrm{~kJ} / \mathrm{mol}$. This marks the enthalpy of formation for a desirable metal hydride system (Andreasen, 2004).
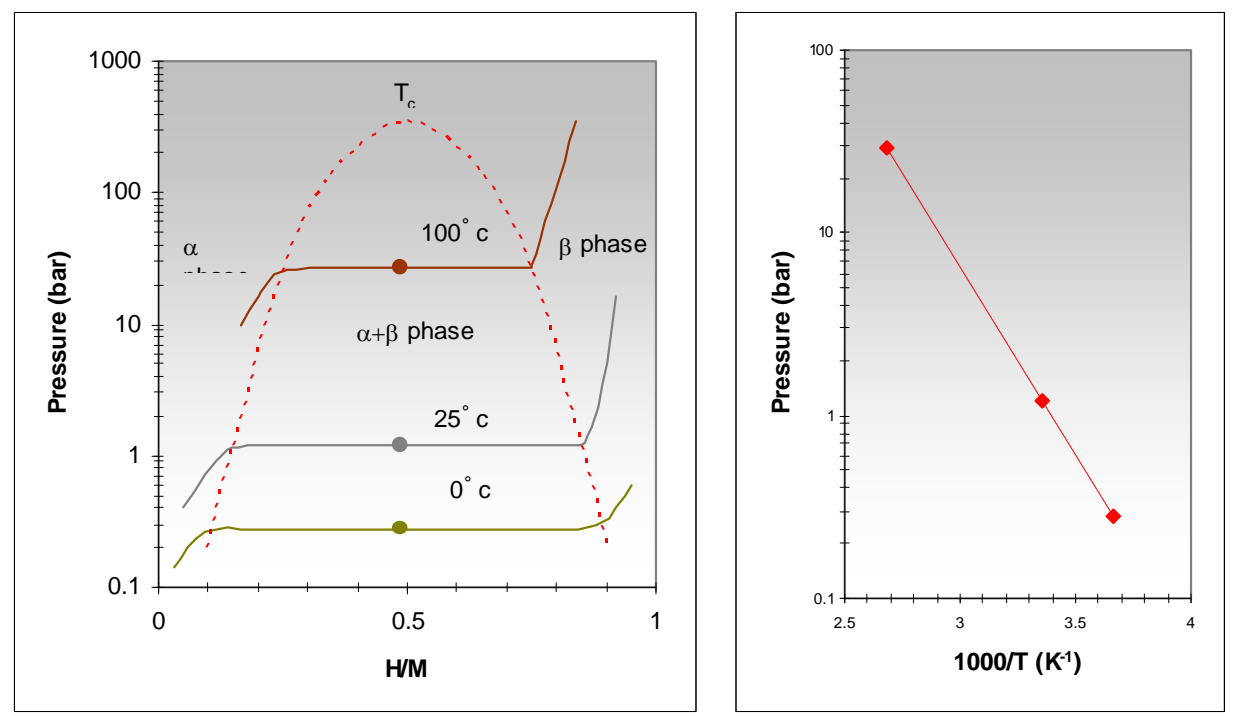

Figure D.2 A typical Pressure-Concentration-Isotherms plot for a metal hydride and the Van't Hoff curve (reproduced from Schlapbach, 2001)

\section{References}

Andreasen Anders, Predicting Formation Enthalpies of Metal Hydrides, Ris $\varnothing$ National Laboratory, Roskilde, Denmark, 2004

Cooper D., C. Y. Wu, D. Yasensky, D. Butt, M. Cai, Hydrogen Storage Characteristics of Nickel Nanoparticle Coated Magnesium Prepared by Dry Particle Coating, KONA, Number 23, 2005, 139-151

Fernández J. F., C. R. Sanchez, Rate Determining Step in the Absorption and Desorption of Hydrogen by Magnesium, Journal of Alloys and Compounds, Volume 340, 2002, pp. 189-198

Koch Carl C., Nanostructured Materials, William Andrew, 2007

Schlapbach Louis, Andreas Züttel, Hydrogen-Storage Materials for Mobile Applications, Nature, Volume 414, 15 November 2001, pp. 353-358

Züttel A., Ch. Nutzenadel, G. Schmid, Ch. Emmenegger, P. Sudan, L. Schlapbach, Thermodynamic Aspects of the Interaction of Hydrogen with Pd Clusters, Applied surface Science, Volume 162-163, 2000, pp. 571-575

Züttel A., Materials for Hydrogen Storage, Materials Today, September 2003, pp. 24-33 


\section{E.1 Surface Free Energy of Elements}

Table E.1 The value of $\gamma_{i}^{0}$ (surface free energy) at $298.2 \mathrm{~K}$ and at $T_{m}$ (Mezey and Giber, 1982)

\begin{tabular}{|c|c|c|c|}
\hline Name & $\boldsymbol{T}_{\boldsymbol{m}}(\mathbf{K})$ & $\left.\gamma_{i}^{\circ}(298.2 \mathrm{~K}) \mathbf{( J m}^{-2}\right)$ & $\left.\gamma_{i}^{\circ}\left(T_{m}\right) \mathbf{( J m}^{-2}\right)$ \\
\hline$A g$ & 1323 & 1.302 & 1.046 \\
\hline$A u$ & 1337 & 1.626 & 1.345 \\
\hline$C o$ & 1768 & 2.709 & 2.003 \\
\hline$C r$ & 2130 & 2.056 & 1.913 \\
\hline$C u$ & 1357 & 1.934 & 1.576 \\
\hline$M g$ & 922 & 0.803 & 0.562 \\
\hline$N b$ & 2741 & 2.983 & 2.022 \\
\hline$P d$ & 1825 & 2.043 & 1.376 \\
\hline$P t$ & 2045 & 2.691 & 2.055 \\
\hline$S b$ & 904 & 0.458 & 0.386 \\
\hline$S n$ & 505 & 0.821 & 0.619 \\
\hline$W$ & 3683 & 3.468 & 2.487 \\
\hline
\end{tabular}

\section{E.2 Vapor Pressure of the Elements}

Table E.2 Vapor pressure of the elements $\log P=A . T^{-1}+B \log T+C T+D(\mathrm{mmHg})$

\begin{tabular}{|c|c|c|c|c|c|c|}
\hline \multirow[t]{2}{*}{ Substance } & \multicolumn{4}{|c|}{$\log P(\mathrm{mmHg})$} & \multirow{2}{*}{$\begin{array}{l}\text { Melting } \\
\text { Point (K) }\end{array}$} & \multirow{2}{*}{$\begin{array}{c}\text { Temp. } \\
\text { range (K) }\end{array}$} \\
\hline & $\mathbf{A}$ & B & $\mathrm{C} \times 10^{3}$ & D & & \\
\hline $\mathbf{A g}$ & $-14,900$ & -0.85 & - & 12.20 & 1235 & 298-m.p. \\
\hline Au & $-19,820$ & -0.306 & -0.16 & 10.81 & 1337 & 298-m.p. \\
\hline Co & $-22,209$ & - & -0.223 & 10.817 & 1768 & 298-m.p. \\
\hline $\mathbf{C r}$ & $-20,680$ & -1.31 & - & 14.56 & 2180 & 298-m.p. \\
\hline $\mathbf{C u}$ & $-17,770$ & -0.86 & - & 12.29 & 1357 & 298-m.p. \\
\hline Pd & $-19,800$ & -0.755 & - & 11.82 & 1828 & 298-m.p. \\
\hline $\mathbf{P t}$ & $-28,460$ & -1.27 & - & 14.33 & 2041 & 298-m.p. \\
\hline W & $-44,000$ & +0.50 & - & 8.76 & 3695 & 298-m.p. \\
\hline
\end{tabular}

(Kubaschewski and Alcock, 1979)

\section{E.3 Cubic Crystal Structures}

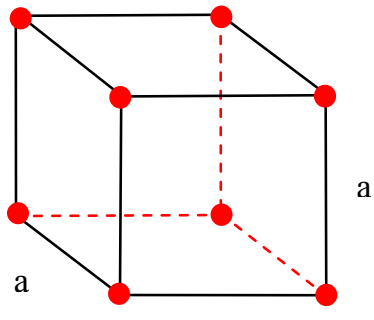

a

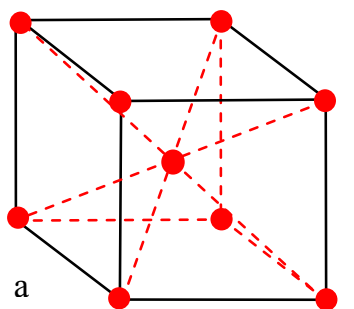

a

(b)

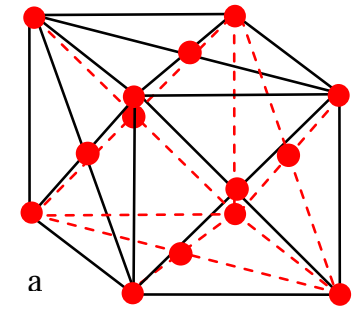

a

(c)

Figure E.1 Simple (a), body centered (b), faced centered (c) cubic structures 
Table E.3 Cubic crystal structures properties

\begin{tabular}{|l|l|l|}
\hline Crystal Structure & Number of atoms per unit cell & $\begin{array}{l}\text { Relationship between Lattice } \\
\text { constant and atomic radius }\end{array}$ \\
\hline Simple cubic & 1 & $\mathrm{a}=2 \mathrm{R}$ \\
\hline Body centered cubic (bcc) & 2 & $a=\frac{4 R}{\sqrt{3}}$ \\
\hline Face centered cubic (fcc) & 4 & $a=2 R \sqrt{2}$ \\
\hline
\end{tabular}

Interplanar spacing: $\quad d=\frac{a}{\sqrt{h^{2}+k^{2}+l^{2}}}$

Ratio of interplanar spacing between two different planes: $\quad \frac{d_{2}}{d_{1}}=\frac{\sqrt{h_{1}^{2}+k_{1}^{2}+l_{1}^{2}}}{\sqrt{h_{2}^{2}+k_{2}^{2}+l_{2}^{2}}}$

Angle between two planes: $\quad \operatorname{Cos} \theta=\frac{h_{1} h_{2}+k_{1} k_{2}+l_{1} l_{2}}{\sqrt{h_{1}^{2}+k_{1}^{2}+l_{1}^{2}} \sqrt{h_{2}^{2}+k_{2}^{2}+l_{2}^{2}}}$

Allowed reflections for FCC only from all even or all odd h, k, 1

Allowed reflections for BCC only from $\mathrm{h}+\mathrm{k}+\mathrm{l}=\mathrm{even}$

\section{E.4 Phase Diagram of Binary Systems}

(Massalski, and Hiroaki, 1990)

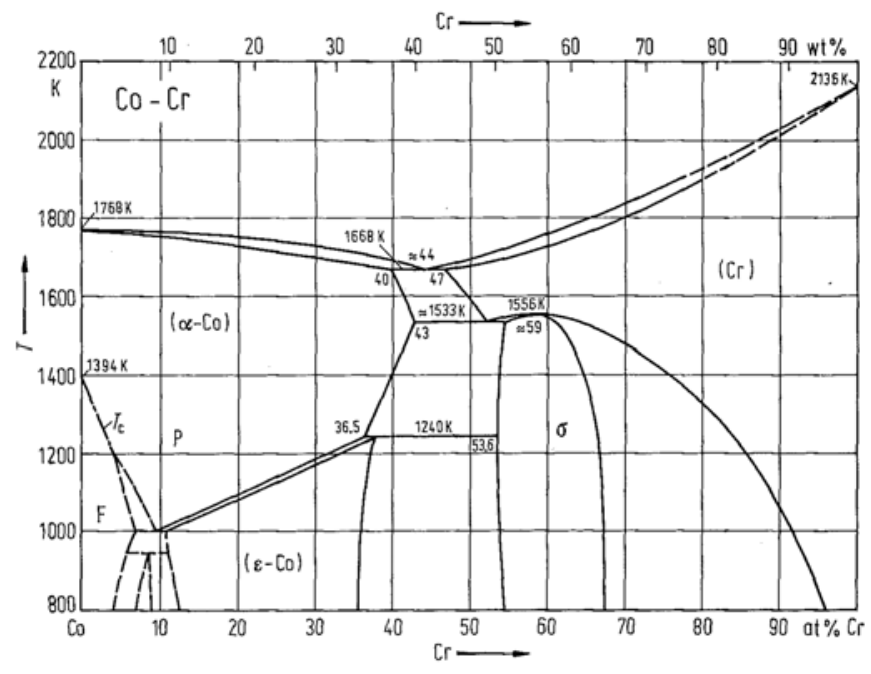

Figure E.2 Co-Cr Phase diagram. P: paramagnetic $F$ : ferromagnetic

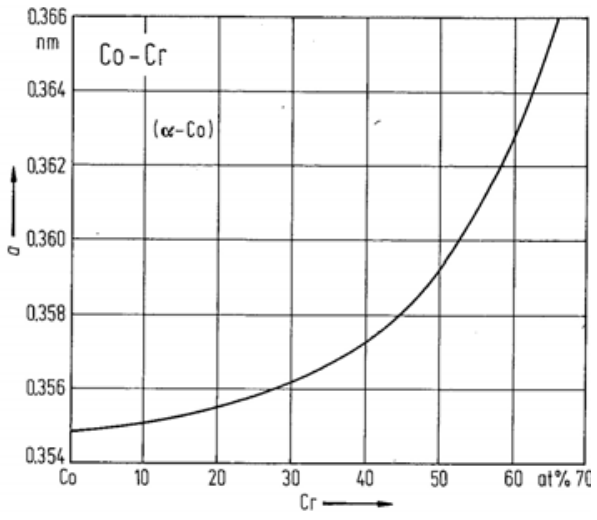

Figure E.3 Co-Cr Lattice parameter vs. composition for $f c c(\alpha-C o)$ solid solutions 


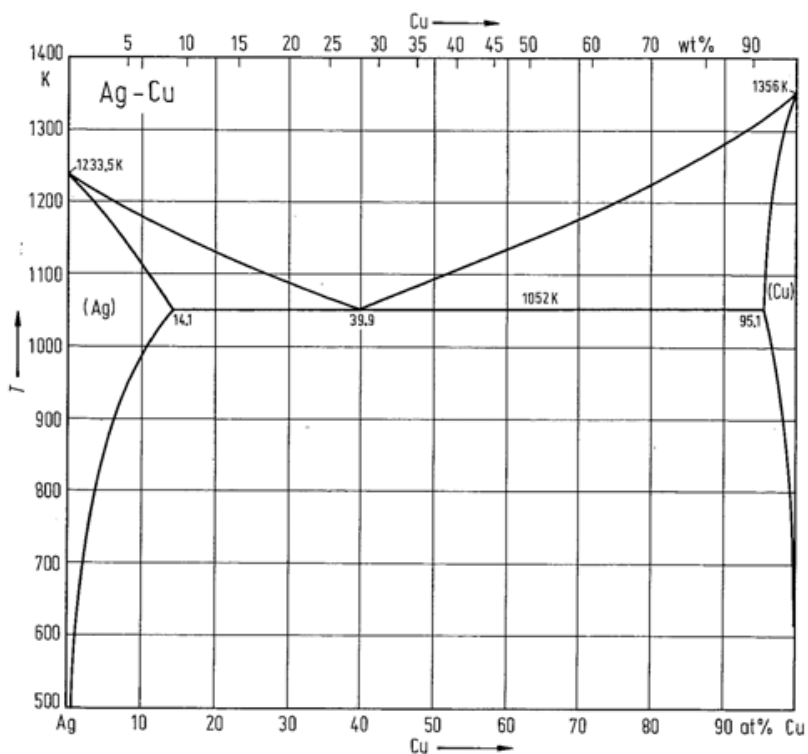

Figure E.4 Ag-Cu Phase diagram

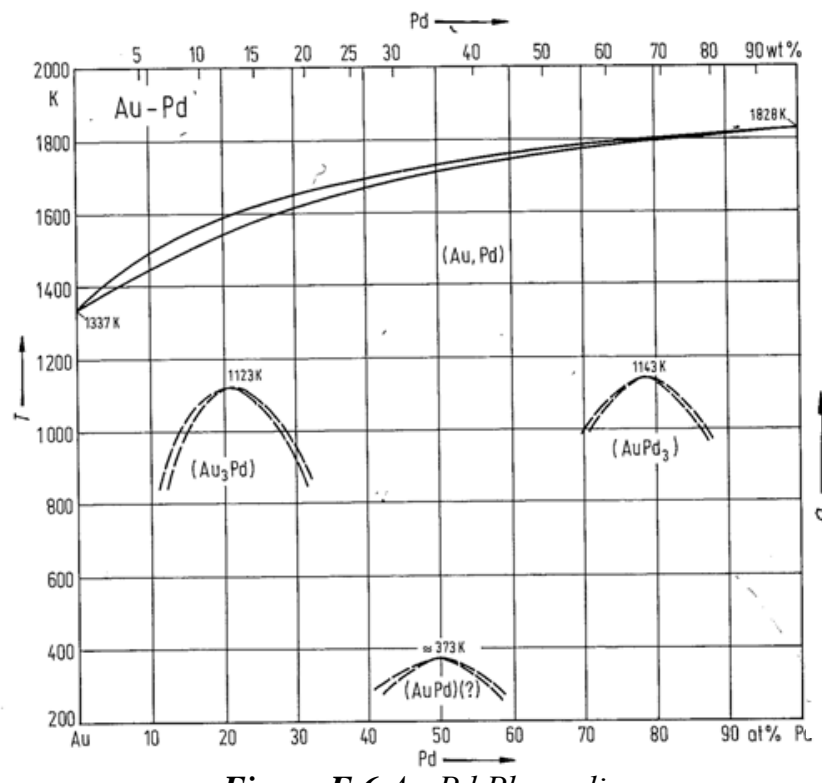

Figure E.6 Au-Pd Phase diagram

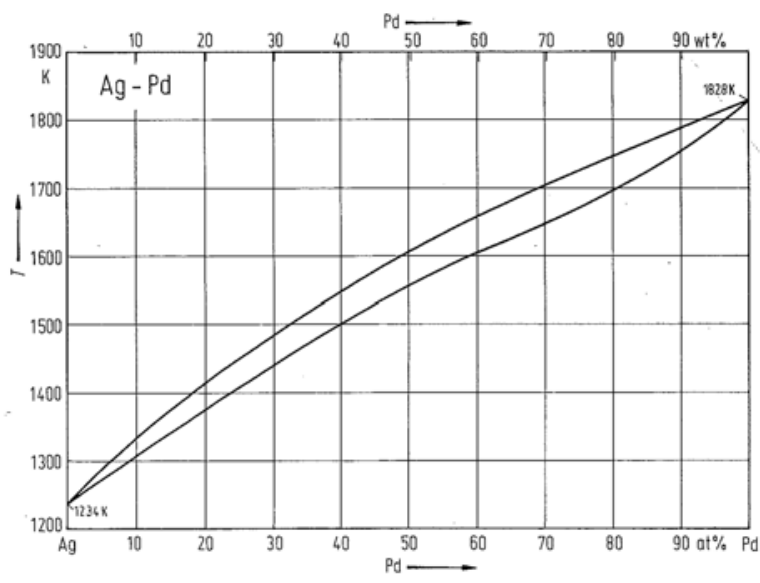

Figure E.8 Ag-Pd Phase diagram

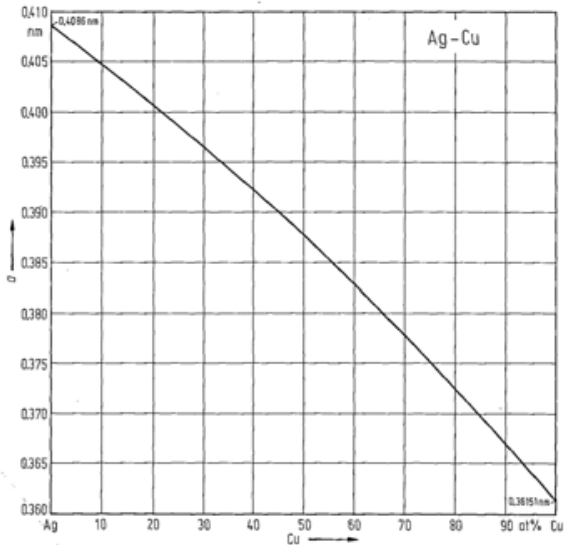

Figure E.5 Ag-Cu Lattice parameter vs. composition for the metastable solid solution

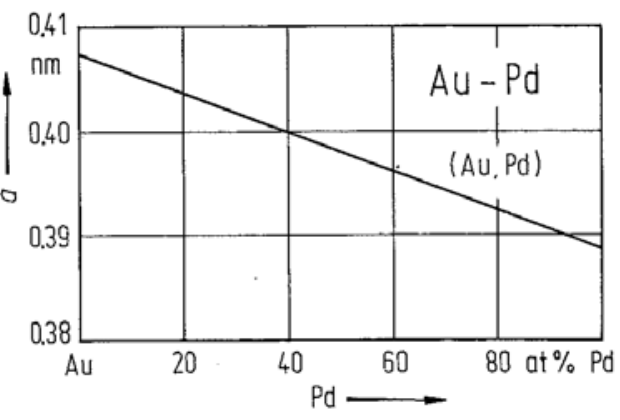

Figure E.7 Au-Pd Lattice parameter of (Au-Pd) solid solution vs. composition

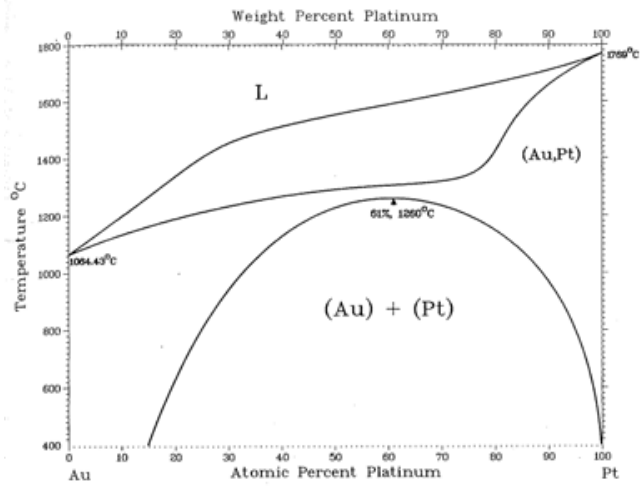

Figure E.9 Au-Pt Phase diagram 


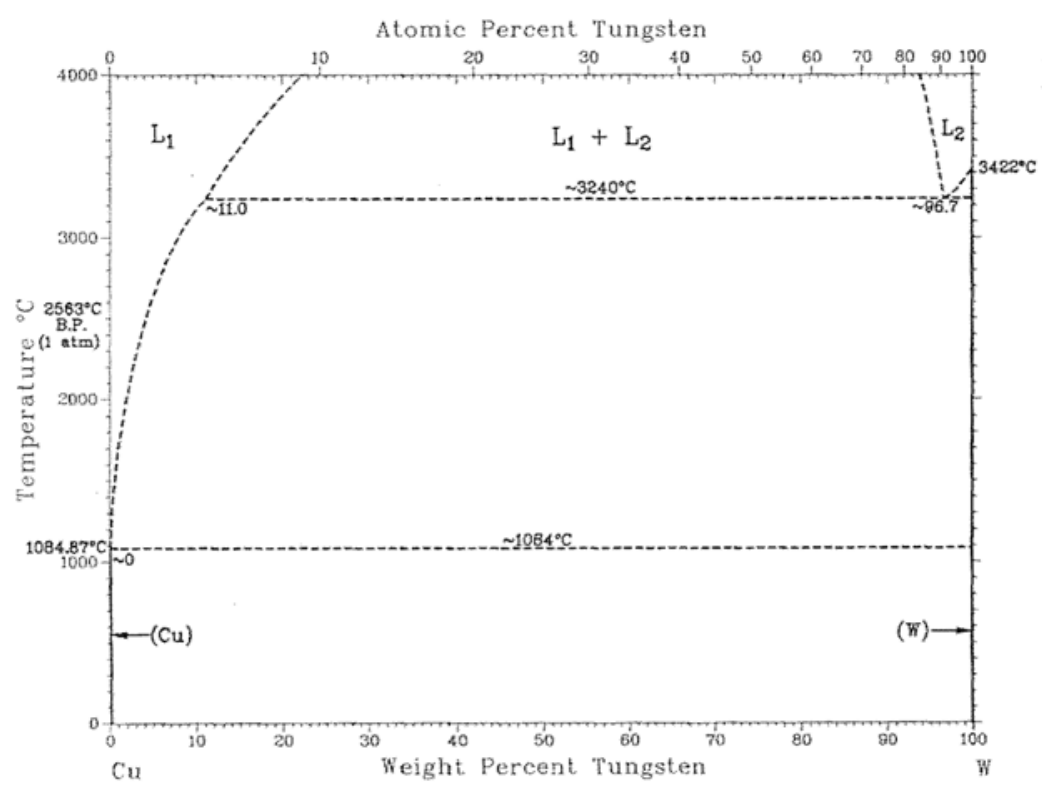

Figure E.10 Cu-W Phase diagram

\section{References}

Kubaschewski O., C. B. Alcock, Metallurgical Thermo-Chemistry, International Series on Materials Science and Technology, Volume 24, Pergamon International Library, 1979

Massalski, Thaddeus B., Okamito Hiroaki, Binary Alloy Phase Diagrams, Volume 2, Metals Park, ASM International, 1990

Mezey L. Z., J. Giber, the Surface Free Energies of Solid Chemical Elements: Calculation from Internal Free Enthalpies of Atomization, Japanese Journal of Applied Physics, Volume 21, Number 11, 1982, pp. 1569-1571

Predel B., O. Madelung, Phase Equilibria, Crystallographic and Thermodynamic Data of Binary Alloys, Belrin: Springer, 1998 


\section{F.1 Analytical Solutions}

Analytical solution for the average particle volume $(\bar{v})$ :

$\left\{\begin{array}{l}\frac{d \bar{v}}{d t}=\frac{1}{2} \alpha\left(\frac{6 k T}{\rho_{p}}\right)^{1 / 2}\left(\frac{3}{4 \pi}\right)^{1 / 6} \Phi \bar{v}^{1 / 6} \\ \bar{v}(0)=v_{0}\end{array}\right.$
$A=\frac{1}{2} \alpha\left(\frac{6 k}{\rho_{p}}\right)^{1 / 2}\left(\frac{3}{4 \pi}\right)^{1 / 6} \Phi \quad$ constant

assuming that the cooling rate $(\kappa)$ is constant and the temperature varies linearly with time:

$T=T_{\circ}-\kappa t$

$\frac{d \bar{v}}{d t}=A\left(T_{\circ}-\kappa t\right)^{1 / 2} \bar{v}^{1 / 6}$

$\frac{d \bar{v}}{\bar{v}^{1 / 6}}=A\left(T_{\circ}-\kappa t\right)^{1 / 2} d t$

$\int \bar{v}^{-1 / 6} d \bar{v}=\int A\left(T_{\circ}-\kappa t\right)^{1 / 2} d t$

$\frac{6}{5} \bar{v}^{5 / 6}=A\left[-\frac{2}{3 \kappa}\left(T_{0}-\kappa t\right)^{3 / 2}\right]+C \quad$ applying initial condition, $\mathrm{C}$ is found

$C=\frac{6}{5} v_{\circ}^{5 / 6}+A \frac{2}{3 \kappa} T_{\circ}^{3 / 2}$

$\bar{v}=\left[-\frac{5 A}{9 \kappa}\left(T_{\circ}-\kappa t\right)^{3 / 2}+\frac{5 A}{9 \kappa} T_{0}^{3 / 2}+v_{0}^{5 / 6}\right]^{6 / 5}$

Analytical solution for the dimensionless excess surface area $(\theta)$ :

Using equation (6.7), the variable $t$ in equation (6.1) can be change to $(\bar{v})$ and equation (6.1) will be converted to :

$$
\begin{aligned}
& \left\{\begin{array}{l}
3 \bar{v} \frac{d \theta}{d \bar{v}}+\left[\frac{\tau_{c}(\bar{v})}{\tau_{f}(\bar{v})}-1\right] \theta=1 \\
\theta\left(v_{\circ}\right)=0
\end{array}\right. \\
& \frac{d \theta}{d \bar{v}}+\frac{1}{3 \bar{v}}\left[\frac{\tau_{c}(\bar{v})}{\tau_{f}(\bar{v})}-1\right] \theta=\frac{1}{3 \bar{v}}
\end{aligned}
$$

the integrating factor is (Miller, 1987): 
$G(\bar{v})=\int \frac{1}{3 \bar{v}}\left[\frac{\tau_{c}(\bar{v})}{\tau_{f}(\bar{v})}-1\right] d \bar{v} \quad \quad$ multiplying the above equation by $\left(e^{G(\bar{v})}\right)$ :

$e^{G(\bar{v})} \frac{d \theta}{d \bar{v}}+e^{G(\bar{v})} \frac{1}{3 \bar{v}}\left[\frac{\tau_{c}(\bar{v})}{\tau_{f}(\bar{v})}-1\right] \theta=\frac{1}{3 \bar{v}} e^{G(\bar{v})}$

$\left[e^{G(\bar{v})} \theta\right]^{\prime}=\frac{1}{3 \bar{v}} e^{G(\bar{v})}$

$e^{G(\bar{v})} \theta=\int \frac{1}{3 \bar{v}} e^{G(\bar{v})} d \bar{v}+C$

$\theta=e^{-G(\bar{v})}\left[\int \frac{1}{3 \bar{v}} e^{G(\bar{v})} d \bar{v}+C\right]$

\section{F.2 Numerical Solution}

Classical fourth-order Runge-Kutta method (Miller, 1987):

$$
\begin{aligned}
& k_{1 n}=h f\left(x_{n}, y_{n}\right) \\
& k_{2 n}=h f\left(x_{n}+\frac{h}{2}, y_{n}+\frac{1}{2} k_{1 n}\right) \\
& k_{3 n}=h f\left(x_{n}+\frac{h}{2}, y_{n}+\frac{1}{2} k_{2 n}\right) \\
& k_{4 n}=h f\left(x_{n}+h, y_{n}+k_{3 n}\right)
\end{aligned}
$$

and

$y_{n+1}=y_{n}+\frac{1}{6}\left(k_{1 n}+2 k_{2 n}+2 k_{3 n}+k_{4 n}\right)$

\section{Reference}

Miller Richard K., Introduction to Differential Equations, Prentice-Hall International Editions, 1987 


\section{Acknowledgement}

Now that the present thesis has come to the end, I would like to take the opportunity to express my sincere gratitude to all the people who helped me with the accomplishment of this work.

First of all, I am very grateful to Prof. Schmidt-Ott who accepted me in his group and gave me the chance to pursue a doctorate degree. It was a pleasure to spend these years working under his supervision. Throughout this period, I was given absolute freedom in finding my way however at the times of despair, he came positively to help with his sharp insight and deep knowledge. I highly value all the experiences and knowledge that I gained in his group.

I wish to express my deep sense of gratitude to Prof. Picken, Prof. Burtscher, Prof. Dunn, Prof. Kruis, Dr. Eijt, Dr. van de Krol for taking part in my examination committee, for the time they spent on reading the manuscript, and for their constructive comments.

I particularly thank Prof. Burtscher whose sabbatical in the Nano Lab, gave our group the opportunity to benefit greatly from his profound knowledge and experimental skills.

I am obliged to Dr. Eijt who facilitated my access to TDS, and also to Dr. Kelder for the collaboration in the early stages of the present thesis.

In this work, particle characterization was of crucial importance. Without the contribution of various experts at TUDelft, this work could never have been realized. I am sincerely thankful to Qiang Xu (STEM), Niek van der Pers (XRD), Kees Kwakernaak (XPS), Ugo Lafont (TEM), Patricia J. Kooyman (TEM), Joop Padmos (ICP), Sander Brouwer (BET), Johan Groen (BET), Walter Legerstee (TDS), Marcel Bus (AFM), Valerie Butselaar (PCS), Gerard de Vos (SEM), Piet Droppert (electrical conductivity).

I would like to appreciate the DCT workshop for their excellent services and friendly help throughout the period of this work- Alex Ploegman, Nico Alberts, as well as Alex Boot whose great sense of humor removed all the troubles I often encountered with the high voltage induced disturbances. Without their help I could never succeed.

Many thanks to Fieneke Spaans, Wil Stolwijk, Karin Wilhelm for their administrative help, in particular their kind efforts in sorting out my visa.

I am also very grateful to Viviane dos Santos Barbosa and Brenda Gharibaan for their valuable help in translating the thesis summary and propositions into Dutch.

I thank Miren Etxabe Elustondo who studied the spark discharge generator in the framework of her master's thesis and helped me with the particle size distribution measurements.

Special thanks to Christian Peineke whose knowledge in physics and electronics combined with his intelligence was always a great source of help.

I have always enjoyed our fruitful discussions with Vincent Vons who had also contribution to studying the oscillating behavior of the spark discharge and particle size distribution measurements. I truly appreciate his assistance. 
Thanks to Dr. Caner Yurteri for our frequent, interesting discussions and also for his help in applying COMSOL.

I would like to acknowledge the present and former members of NSM group for making this period so wonderful and memorable- Jan van Erven, Bas van Laarhoven, Ineke Kleefsman, Tomasz M. Trzeciak, Urszula Stachewicz, Ajay Galdhar, Maria Margarita Vivas, Raghuraman Pitchumani, Orion J. P. Mouraille, Sjaak Verdoold, Micha-Klaus Mueller, Tobias Pfeiffer, John Juan, Khalid Chougrani, Mark G. Ullmann, Bart van der Graaf to name a few. It was also an honor to be in the same group with Drs. Henk Nugteren, Dr. Jan C. M. Marijnissen, and Prof. Stefan Luding.

Many thanks to my room mates Anna Hubacz and Yonsuang Arnanthigo for all the joyful moments we spent together and also for listening to me during my difficult times.

I am profoundly indebted to my siblings who took a constant and affectionate care of my beloved mother during my absence. My words certainly fail to thank them adequately.

Finally, my heartiest gratitude to my dear children, Maryam and Ali, whom I am very proud of, and to my dear husband and my best friend Ata, for all their understanding, patience and love which kept me going... 